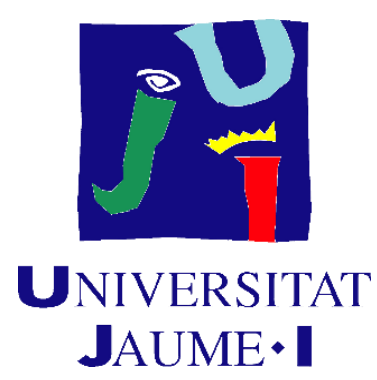

Universitat Jaume I Doctoral School

Doctoral Programme in Sciences

\title{
Device Modelling of Perovskite Solar Cells using Small Perturbation Methods
}

Report submitted in order to be eligible for a doctoral degree awarded by the Universitat Jaume I

Author

Sandheep Pulya Ravishankar
Director

Juan Bisquert Mascarell

Castelló de la Plana, June, 2019 


\section{Funding}

The following sources were responsible for funding the research carried out in this thesis.

- Santiago Grisolia Grant for foreign researchers provided by Generalitat Valenciana, grant number GRISOLIA/2014/034

- Project - "Perovskitas fotovoltaicas establizadas de alto rendimiento" from MINECO, Spain, reference number: MAT2016-76892-C3-1-R

- Project - "Promoción de los proyectos europeos", reference number: 161314 


\begin{abstract}
Perovskite solar cells (PSCs) have shown large power conversion efficiencies in the laboratory, comparable to Silicon-based solar cells, in a very short time since their inception. However, a major impediment to their commercialization is their low stability in ambient environmental conditions and low tolerance to humidity. This instability is deeply connected to the mixed ionic-electronic conduction in PSCs, which is manifested in the hysteresis observed in current-voltage curves of the PSC, which depends strongly on external parameters of measurement. Therefore, this thesis aims to develop a holistic electrical model of PSCs using kinetic information to understand recombination, charge accumulation and transport of electronic carriers and ions.

Existing models in the literature model the PSC similar to an organic solar cell, with a net electric field through the absorber that is the difference between the workfunctions (WF) of the two selective contacts. This electric field is modulated by the ions and determines the open circuit voltage $\left(V_{o c}\right)$ via the built-in voltage $\left(V_{b i}\right)$, therefore being mainly a charge collection model. However, this model cannot account for several types of hysteresis observed and also the giant capacitance that scales exponentially with voltage and light intensity, far larger than a purely ionic Helmholtz capacitance. Based on existing observations of an extremely slow decay of the $V_{o c}$ and a persistent photovoltage, combined with huge charge densities visualised at the electron selective contact (ESL)/perovskite interface, a kinetic model based on the formation of an accumulation of both electronic and ionic carriers at the ESL/perovskite interface at large forward bias is proposed. This recombination based model connects the internal and external voltage of the PSC via the slow movement of the ions, which reproduces several of the hysteretic trends and their dependence on external parameters of voltage, scan speed and light intensity. The model is then extended to include surface recombination between the accumulated holes and electrons in the ESL, which depends strongly on the properties of the compact $\mathrm{TiO}_{2}$ layer. The interplay between charge accumulation and surface recombination explains the unique $V_{o c}$ hysteresis observed in triple layer mesoscopic PSCs. The validity of the model depends largely on the nature and quality of transport in the PSC, which is then investigated by a two-pronged approach. The first method involves investigating the dependence of the ESL WF on the obtained $V_{o c}$ for high efficiency PSCs. A WF variation of over $1 \mathrm{eV}$ yielded very similar $V_{o c}$ values and almost identical $j_{s c}$ values, indicating that the $V_{b i}$ does not control the $V_{o c}$ and that transport in the PSC is predominantly diffusive, with potential drops likely being absorbed completely at the interfaces.

After establishing that recombination is the dominant mechanism controlling the performance of the PSC, Impedance Spectroscopy (IS) was used to identify the internal separation of potential (ie alternate recombination pathways) in the PSC. Due to the large disparity in results in IS that depends on materials and methods, including the general lack of information obtained in the case of PSCs, Intensity Modulated Photocurrent
\end{abstract}


Spectroscopy (IMPS) measurements were also carried out. From existing knowledge about the IS equivalent circuit (EC) and the large low frequency (LF) capacitance observed, a basic theory of IMPS for PSCs was developed to interpret the IMPS spectra, with an internal separation of photovoltage across resistances in the EC yielding a unique reduction in the external quantum efficiency (EQE) at low frequencies between $10 \mathrm{~Hz}-$ $10 \mathrm{mHz}$. This effect was also directly measured from the standard method of measuring the EQE using the differential spectral response method, where chopper frequencies between $10-500 \mathrm{~Hz}$ yield a variation as large as $10 \%$ in the measured EQE at short circuit (SC) conditions. In addition, the nature of the elements of the EC forming the time constants in either quadrant in an IMPS Q-plane plot is clarified through detailed derivations and simulations. Finally, IMPS and IS measurements were carried out at OC conditions to develop the underlying EC using information and parameter matching from both small perturbation techniques. This allows for the observation of an extra capacitance from IMPS measurements that is invisible in IS measurements due to the different elements forming time constants in either technique. This capacitance is two orders smaller than the giant LF capacitance, whose charging resistance is critical at controlling the net device resistance at standard voltage scan rates for measuring the PSC performance. The developed EC reproduces both the IS and IMPS response of PSCs, taking a large stride forward in creating a robust EC for the PSC that is generally subject to large variation in response based on materials and design. 


\section{Declaration}

I hereby declare that this thesis contains no material that has been used to obtain any other degree or certification of any kind and that all the material used has not been published by any other author, unless due reference has been made. The co-authors of the publications arising from this work have waived their right to present them as part of any other $\mathrm{PhD}$ thesis.

The research work for this thesis was carried out at the Institute of Advanced Materials (INAM), Department of Physics, Universitat Jaume I in Castellon de la Plana, Spain, under the direction of Prof. Juan Bisquert, who served as both supervisor and tutor.

I also confirm that reasonable effort has been made to obtain copyright permissions for third-party content included in this thesis and any omissions with regards to this are unknowing and purely accidental. 


\section{Acknowledgements}

First and foremost, I would like to thank my thesis supervisor Prof. Juan Bisquert for his guidance throughout the tenure of this thesis. His deep knowledge of semiconductor device physics and his creativity and passion for problem solving have inspired and influenced this body of work strongly.

Prof. Germà Garcia-Belmonte has also been instrumental to the development of this work, especially in the area of electrical modelling of solar cells. His willingness to impart his knowledge through several discussions throughout the duration of the thesis is highly appreciated.

I would like to acknowledge Prof. Francisco-Fabregat Santiago for his expertise in all things related to Impedance Spectroscopy and his patience in clarifying several doubts regarding the same over several enjoyable discussions.

This thesis would not have been possible without the help of several colleagues at the Institute of Advanced Materials (INAM) and external collaborators. Clara Aranda, who fabricated the perovskite solar cells for most of the experimental work in this thesis and who shares a strong passion for understanding the perovskite solar cell is greatly appreciated. I also thank Dr. Antonio Guerrero for fabricating solar cells for some of the projects. I thank Prof. Mohammad Khaja Nazeeruddin of EPFL Valais, Dr. Pablo Boix at University of Valencia, Prof. Juan Antonio Anta from University of Pablo Olavide and Prof. Shaibal Sarkar at the Indian Institute of Technology Bombay (IITB), for hosting my research visits in their respective groups and ensuring that my stay was pleasant throughout. I would also like to thank Loles Merchan Mundina for her selfless help in sorting out several administrative and legal issues during my stay in Spain.

In addition, I would like to acknowledge several of my colleagues who have become good friends over the course of this thesis. Clara Aranda, Dr. Miguel García Tecedor, Agustin Bou and Dr. Carlos Echeverría Arrondo have made the duration of this thesis and my time in Spain extremely enjoyable, whilst also providing help and support whenever required. I am grateful to my best friends back home, Sudarshan Varadhan and Arun Rengaswamy, for just being who they are. I am also thankful to Neha Sukumar for preparing the excellent cover for this thesis. As an aside, I would like to thank Dr. Lakshmi Vijayakumar for helping me through a very difficult time and Mr. V.B. Chandrasekhar, who taught me to enjoy the process of improving myself everyday.

Finally and most importantly, I would like to dedicate this thesis to my parents, sister and my five dogs who have encouraged me in all my endeavours and provided their love and all forms of support unconditionally. 


\section{List of Publications included in this Thesis}

1. Ravishankar, S.; Almora, O.; Echeverría-Arrondo, C.; Ghahremanirad, E.; Aranda, C.; Guerrero, A.; Fabregat-Santiago, F.; Zaban, A.; Garcia-Belmonte, G.; Bisquert, J. Surface Polarization Model for the Dynamic Hysteresis of Perovskite Solar Cells, The Journal of Physical Chemistry Letters, 2017, 8, 915-921. DOI: 10.1021/acs.jpclett.7b00045, Impact Factor: 8.709

2. Rong, Y.; Hu, Y.; Ravishankar, S.; Liu, H.; Hou, X.; Sheng, Y.; Mei, A.; Wang, Q.; Li, D.; Xu, M. Tunable hysteresis effect for perovskite solar cells, Energy \& Environmental Science, 2017, 10, 2383-2391. DOI: 10.1039/C7EE02048A, Impact Factor: 30.067

3. Ravishankar, S.; Gharibzadeh, S.; Roldán-Carmona, C.; Grancini, G.; Lee, Y.; Ralaiarisoa, M.; Asiri, A. M.; Koch, N.; Bisquert, J.; Nazeeruddin, M. K. Influence of Charge Transport Layers on Open-Circuit Voltage and Hysteresis in Perovskite Solar Cells, Joule, 2018, 2, 788-798. DOI: 10.1016/j.joule.2018.02.013

4. Ravishankar, S.; Aranda, C.; Boix, P. P.; Anta, J. A.; Bisquert, J.; GarciaBelmonte, G. Effects of Frequency Dependence of the External Quantum Efficiency of Perovskite Solar Cells, The Journal of Physical Chemistry Letters, 2018, 9, 3099-3104. DOI: 10.1021/acs.jpclett.8b01245, Impact Factor: 8.709

5. Ravishankar, S.; Aranda, C.; Sanchez, S.; Bisquert, J.; Saliba, M.; GarciaBelmonte, G. Perovskite Solar Cell Modeling using Light and Voltage Modulated Techniques, The Journal of Physical Chemistry C, 2019, 123(11), 6444-6449. DOI: 10.1021/acs.jpcc.9b01187, Impact Factor: 4.484

This thesis has been accepted by the co-authors of the publications listed above that have waived the right to present them as part of another $\mathrm{PhD}$ thesis. 


\section{List of Publications not included in this Thesis}

1) Zhang, K.; Ravishankar, S.; Ma, M.; Veerappan, G.; Bisquert, J.; FabregatSantiago, F.; Park, J. H. Overcoming Charge Collection Limitation at Solid/Liquid Interface by a Controllable Crystal Deficient Overlayer, Advanced Energy Materials, 2017, 7, 1600923. DOI: 10.1002/aenm.201600923, Impact Factor: 21.875

2) Lopez-Varo, P.; Jiménez-Tejada, J. A.; García-Rosell, M.; Anta, J. A.; Ravishankar, S.; Bou, A.; Bisquert, J. Effects of Ion Distributions on Charge Collection in Perovskite Solar Cells, ACS Energy Letters, 2017, 2, 1450-1453. DOI: 10.1021/acsenergylett.7b00424, Impact Factor: 12.277

3) Yadav, S. K.; Ravishankar, S.; Pescetelli, S.; Agresti, A.; Fabregat-Santiago, F.; Di Carlo, A. Stability of dye-sensitized solar cells under extended thermal stress, Physical Chemistry Chemical Physics, 2017, 19, 22546-22554. DOI: 10.1039/C7CP04598K, Impact Factor: 3.906

4) Trześniewski, B. J.; Digdaya, I. A.; Nagaki, T.; Ravishankar, S.; Herraiz-Cardona, I.; Vermaas, D. A.; Longo, A.; Gimenez, S.; Smith, W. A. Near-complete suppression of surface losses and total internal quantum efficiency in $\mathrm{BiVO}_{4}$ photoanodes, Energy \& Environmental Science, 2017, 10, 1517-1529. DOI: 10.1039/C6EE03677E, Impact Factor: 30.067

5) Lopez-Varo, P.; Jiménez-Tejada, J. A.; García-Rosell, M.; Ravishankar, S.; Garcia-Belmonte, G.; Bisquert, J.; Almora, O. Device physics of hybrid perovskite solar cells: theory and experiment, Advanced Energy Materials, 2018, 8, 1702772. DOI: 10.1002/aenm.201702772, Impact Factor: 21.875 


\section{Table of Contents}

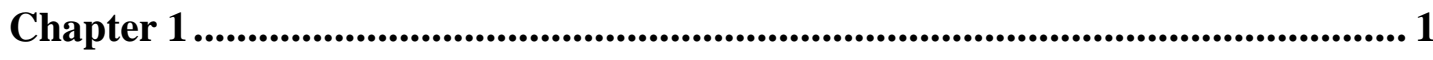

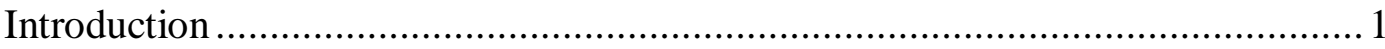

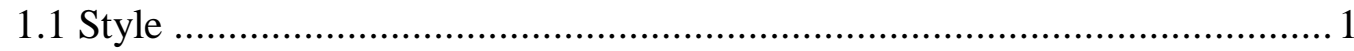

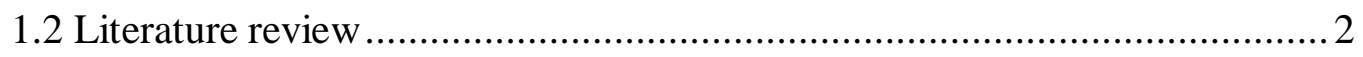

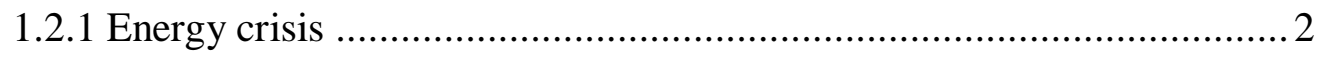

1.2.2 Photovoltaics for the future ....................................................... 3

1.2.3 Perovskite Solar Cells ................................................................. 4

1.2.3.1 Device Structure ................................................................ 4

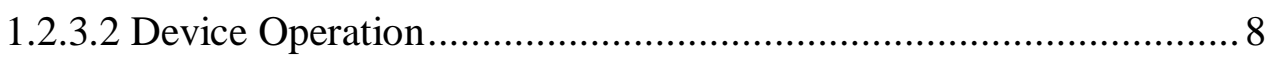

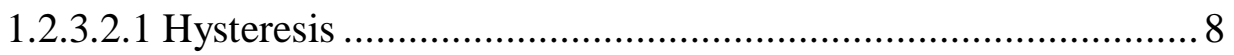

1.2.3.2.2 Effect of voltage preconditioning ...................................... 10

1.2.3.2.3 Effect of light and temperature.......................................... 11

1.2.3.2.4 Effect of Contacts ......................................................... 12

1.2.3.2.5 Recombination - Fast and slow timescales......................... 13

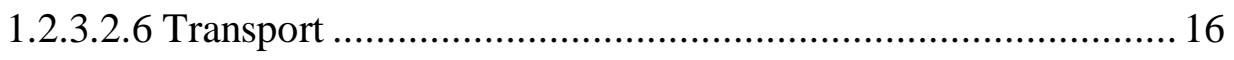

1.2.3.3 Insights from small perturbation methods ................................ 17

1.2.3.3.1 Capacitances and Resistances ............................................. 17

1.2.3.3.2 Photocurrent kinetics ...................................................... 21

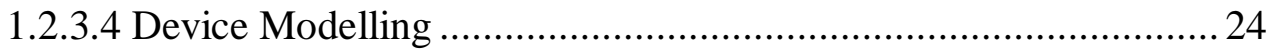

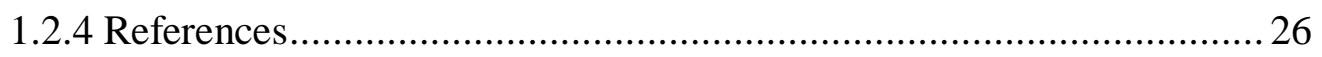

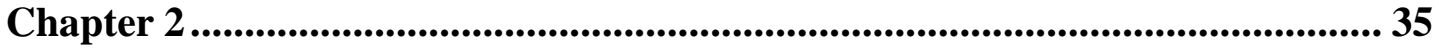

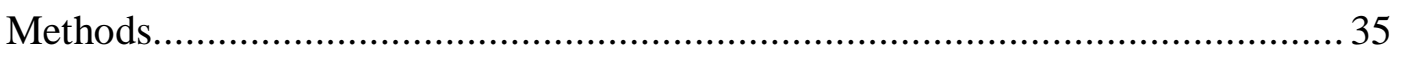

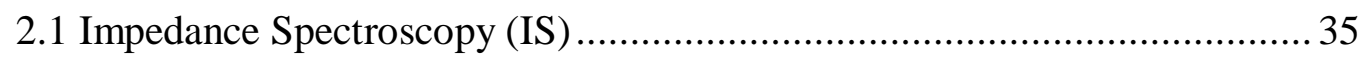

2.2 Intensity Modulated Photocurrent Spectroscopy (IMPS) ........................ 42

2.2.1 Measurement protocols ........................................................... 48

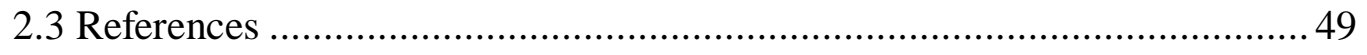

Chapter 3 .................................................................................................... 51

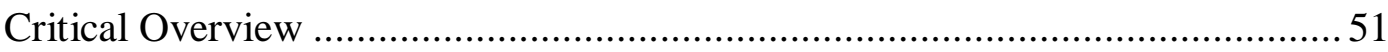

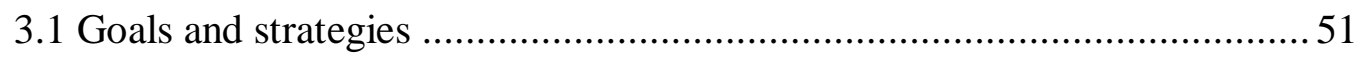

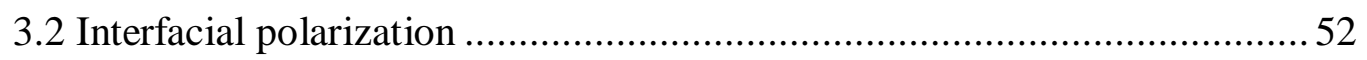

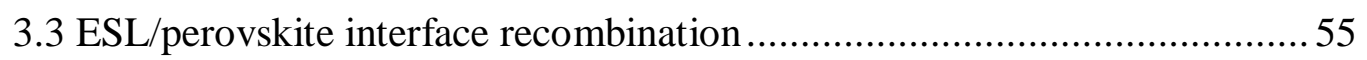

3.4 Role of the built-in electrical field ...........................................................58

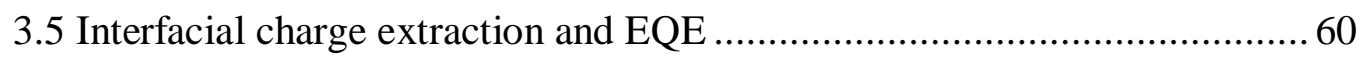

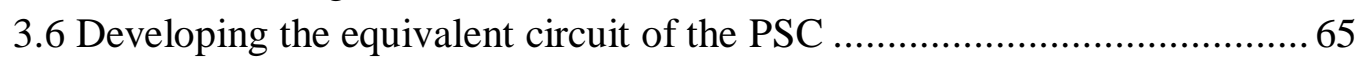

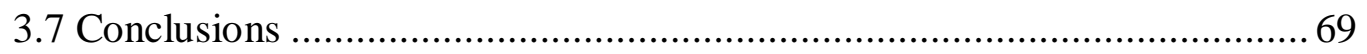

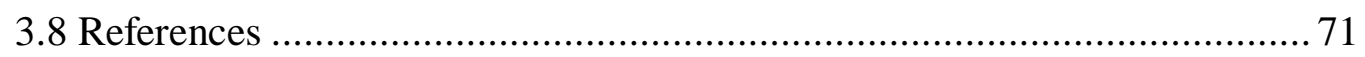

Chapter 4 .......................................................................................................................... 73

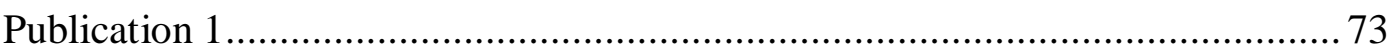

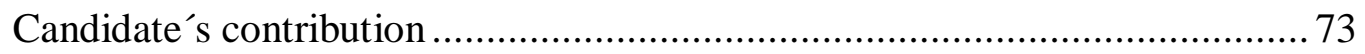




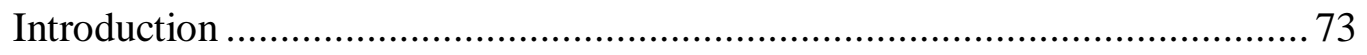

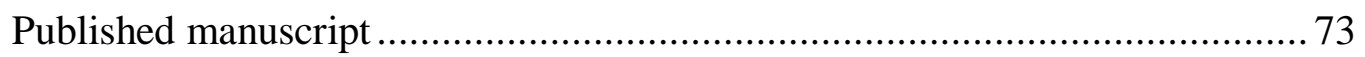

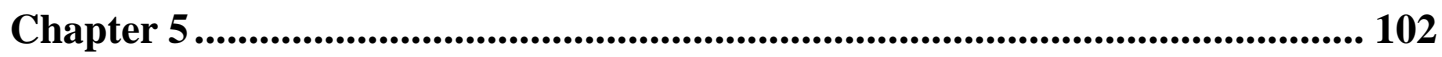

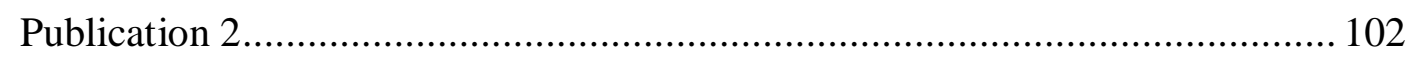

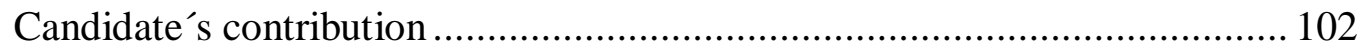

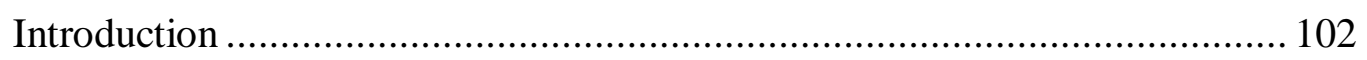

Published manuscript .......................................................................... 102

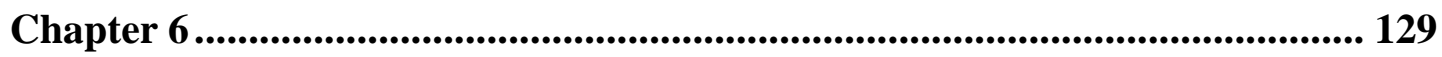

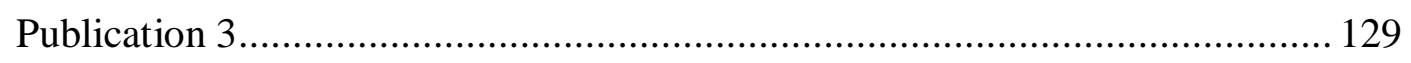

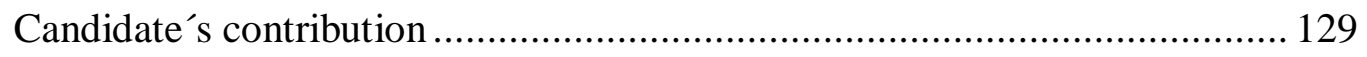

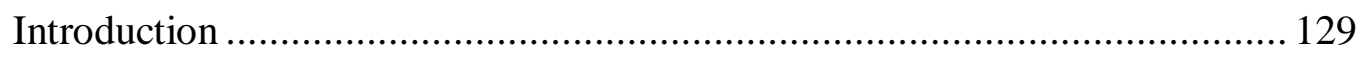

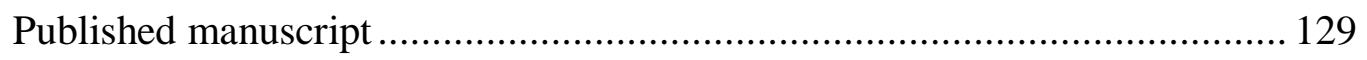

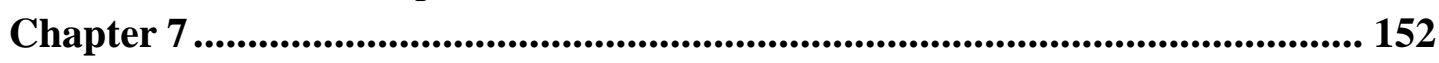

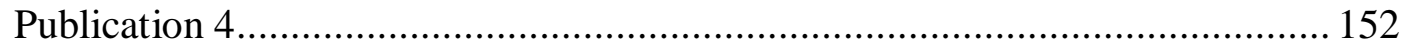

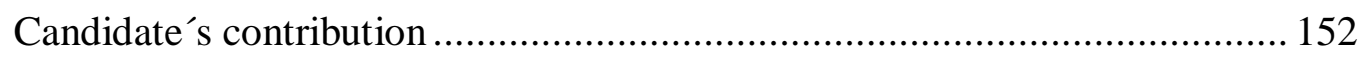

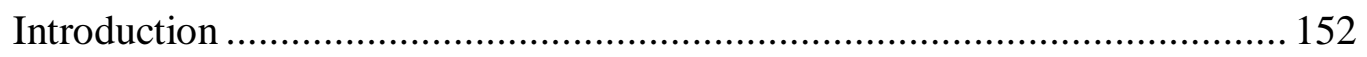

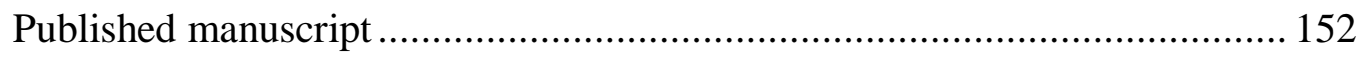

Chapter 8 .................................................................................................. 170

Publication 5..................................................................................... 170

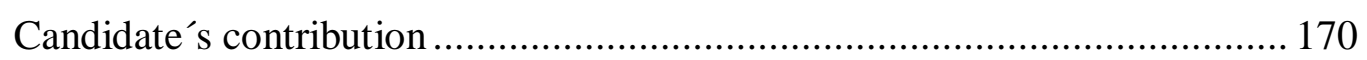

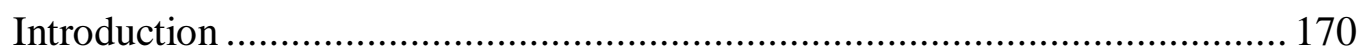

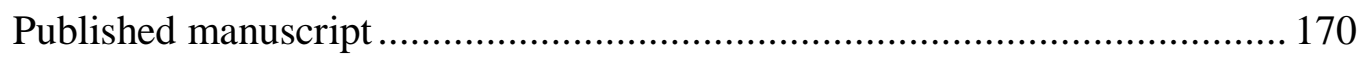

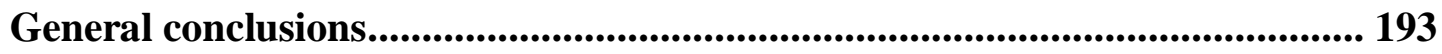




\begin{tabular}{|c|c|}
\hline PSC & Perovskite solar cell \\
\hline DSSC & Dye-sensitized solar cell \\
\hline$V_{o c}$ & Open circuit voltage \\
\hline$V_{b i}$ & Built-in voltage \\
\hline ESL & Electron selective contact \\
\hline HSL & Hole selective contact \\
\hline$j_{S c}$ & Short circuit current \\
\hline IS & Impedance spectroscopy \\
\hline IMPS & Intensity modulated photocurrent spectroscopy \\
\hline $\mathrm{EC}$ & Equivalent circuit \\
\hline $\mathrm{HF}$ & High frequency \\
\hline IF & Intermediate frequency \\
\hline $\mathrm{LF}$ & Low frequency \\
\hline EQE & External quantum efficiency \\
\hline $\mathrm{OC}$ & Open circuit \\
\hline $\mathrm{SC}$ & Short circuit \\
\hline $\mathrm{c}-\mathrm{Si}$ & Crystalline Silicon \\
\hline $\mathrm{m}-\mathrm{Si}$ & Multi-crystalline Silicon \\
\hline $\mathrm{c}-\mathrm{TiO}_{2}$ & Crystalline $\mathrm{TiO}_{2}$ \\
\hline $\mathrm{m}-\mathrm{TiO}_{2}$ & Mesoporous $\mathrm{TiO}_{2}$ \\
\hline DSSC & Dye-sensitized solar cell \\
\hline CIGS & Copper Indium Gallium Selenide \\
\hline PV & Photovoltaics \\
\hline PCBM & {$[6,6]-$ phenyl-C-61-butyric acid methyl ester } \\
\hline Spiro-OMeTAD & $\begin{array}{l}2,2^{\prime}\left(7,7^{\prime}\right) \text {-tetrakis-(N,N-di- } p \text {-methyloxyphenyl- } \\
\text { amine }) 9,9^{\prime} \text { - spirobifluorene }\end{array}$ \\
\hline PEDOT:PSS & poly 4-styrenesulfonate \\
\hline MAPI & Methylammonium Lead Iodide \\
\hline PL & Photoluminescence \\
\hline$j-V$ & Current density - voltage \\
\hline FR & Forward to reverse bias \\
\hline $\mathrm{RF}$ & Reverse to forward bias \\
\hline FF & Fill factor \\
\hline $\mathrm{CB}$ & Conduction band \\
\hline VB & Valence band \\
\hline LED & Light-emitting diode \\
\hline $\mathrm{B}_{\mathrm{rad}}$ & Radiative recombination rate constant \\
\hline$n$ & Electron density \\
\hline
\end{tabular}




\begin{tabular}{|c|c|}
\hline$p$ & Hole density \\
\hline$n_{0}$ & Equilibrium electron concentration \\
\hline$p_{0}$ & Equilibrium hole concentration \\
\hline $\mathrm{N}_{\mathrm{A}}$ & Acceptor dopant density \\
\hline$\tau_{n}$ & Electron recombination lifetime \\
\hline$\tau_{p}$ & Hole recombination lifetime \\
\hline$\tau_{\text {rec }}$ & Minority carrier recombination lifetime \\
\hline$S_{n}$ & Electron surface recombination velocity \\
\hline SRH & Shockley-Read-Hall \\
\hline $\mathrm{U}$ & Recombination rate \\
\hline$D_{n / p}$ & Diffusion coefficient of electrons/holes \\
\hline$\mu_{n / p}$ & Mobility of electrons/holes \\
\hline$E$ & Electrical field \\
\hline$V$ & Electrical potential \\
\hline$E_{f n}$ & Electron Fermi level \\
\hline$E_{f p}$ & Hole Fermi level \\
\hline$L_{d}$ & Diffusion length \\
\hline$Z$ & Impedance \\
\hline$Q$ & IMPS transfer function \\
\hline$p_{b}$ & Bulk majority hole density \\
\hline$C_{S}$ & Surface capacitance \\
\hline$C_{a c c}$ & Accumulation capacitance \\
\hline$C_{g}$ & Bulk dielectric capacitance \\
\hline$R_{\text {rec }}$ & Recombination resistance \\
\hline$R_{S}$ & Series resistance \\
\hline$\omega_{d}$ & Characteristic frequency of diffusion \\
\hline$V_{\text {stab }}$ & Stabilized potential \\
\hline$Z^{\prime}$ & Real part of impedance \\
\hline$Z^{\prime \prime}$ & Imaginary part of impedance \\
\hline$Q^{\prime}$ & Real part of IMPS transfer function \\
\hline$Q^{\prime \prime}$ & Imaginary part of IMPS transfer function \\
\hline$C_{\mu}$ & Chemical capacitance \\
\hline TCO & Transparent conducting Oxide \\
\hline FTO & Fluorine-doped Tin Oxide \\
\hline$L_{n / p}$ & Diffusion length of electrons/holes \\
\hline$\phi$ & Photon flux \\
\hline PEC & Photoelectrochemical \\
\hline Alpha & Absorption coefficient \\
\hline DC & Direct current \\
\hline $\mathrm{AC}$ & Alternating current \\
\hline SPM & Surface polarization model \\
\hline$V_{s}$ & Surface potential \\
\hline
\end{tabular}


$\mathrm{F}$

$\mathrm{R}$

PCE

UPS

WF

IQE

$\sigma$

$R_{t}$

$R_{H F}$

$R_{I F}$

$R_{L F}$

$C_{I F}$

$C_{L F}$

$j_{\phi}$
Forward

Reverse

Photoconversion efficiency

Ultraviolet photoelectron spectroscopy

Workfunction

Internal quantum efficiency

Conductivity

Transport resistance

High frequency resistance

Intermediate frequency resistance

Low frequency resistance

Intermediate frequency capacitance

Low frequency capacitance

Photon flux current density 


\section{List of physical constants}

$\begin{array}{lll}q & \text { Electronic charge } & 1.602 \times 10^{-19} \mathrm{C} \\ k_{B} T & \text { Thermal voltage } & 0.026 \mathrm{~V} \\ h & \text { Planck's constant } & 6.626 \times 10^{-34} \mathrm{kgm}^{2} \mathrm{~s}^{-1} \\ c & \text { Speed of light } & 3 \times 10^{8} \mathrm{~ms}^{-1} \\ \epsilon_{0} & \text { Permittivity of vacuum } & 8.85 \times 10^{-12} \mathrm{Fm}^{-1} \\ \epsilon_{r} & \text { Relative permittivity } & \end{array}$




\section{Chapter 1}

\section{Introduction}

\subsection{Style}

This thesis is written in a journal article compilation style format. This format involves the division of the thesis into several chapters that address specific aspects regarding the work carried out. Chapter 1 involves a review of the literature to summarise the current state of knowledge in the field and open questions that make up the core of the research carried out. This is then followed by a description of the main techniques used to tackle the research problems, including a summary of the history of these techniques with respect to their applications in solar energy conversion, in Chapter 2. Chapter 3 carries out a critical overview of the studied research problems and the main results obtained, describing their context, interpretation and significance. Chapters 4-8 contain the obtained results and their conclusions in the form of the author's final version of the manuscript prior to publication, with a preliminary statement of contribution provided before each publication. Finally, a section of general conclusions summarises all the findings and their implications for the current and future understanding of the field. 


\subsection{Literature review}

\subsubsection{Energy crisis}

One of the biggest problems facing the world today is to satisfy the ever-growing demand for power. By 2040, it is estimated that the world energy consumption will rise by $28 \% .^{1}$ This demand will mainly come from the developing nations of the world, where rapid economic development, electrification and growing populations will occur. This will be coupled to the fact that the rapid advancement in quality and sophistication of devices will result in very high average energy consumption per device in the near future. Furthermore, current energy supply is dominated by fossil fuels such as oil and natural gas, whose reserves are estimated to last until 2040 and 2042 respectively. ${ }^{2}$ In addition, with the looming threat of global warming, aided by polluting $\mathrm{CO}_{2}$ emissions from burning fuel sources such as oil and coal, there is an urgent need for clean energy as well.

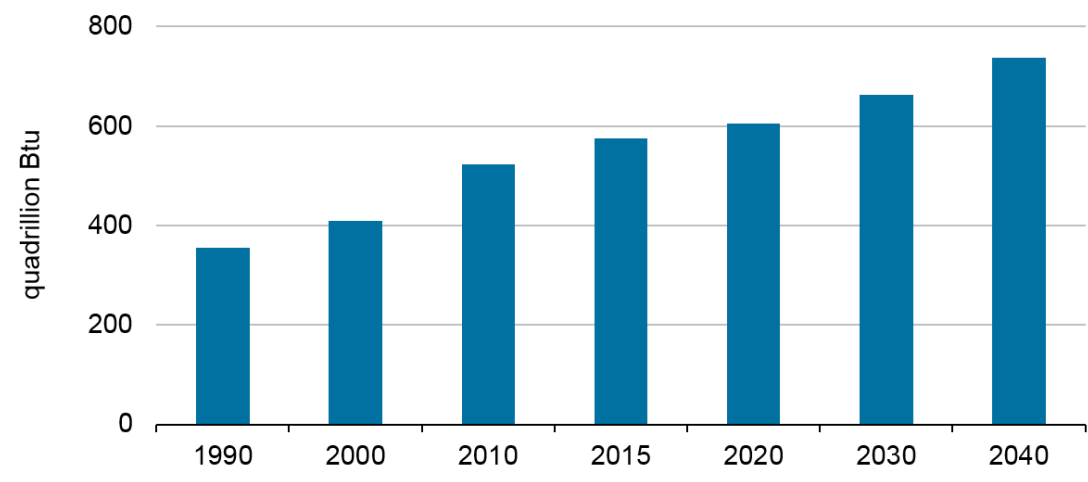

Figure 1.1 Projected world energy consumption (from 2015) in quadrillion British thermal units $(\mathrm{Btu})(1 \mathrm{Btu}=1055 \mathrm{~J})$, extrapolated to the year 2040. Data adapted from Ref. 1.

Therefore, it is imperative that we harness and maximise the production of clean energy through renewable energy sources such as wind, hydroelectric, geothermal and solar energy. Currently, renewable energy sources form only $13.7 \%$ of the global energy produce, with biomass fuels contributing a large share. ${ }^{3}$ Increasing the production capacity of these renewable sources will not only increase the efficiency of energy production by phasing out inefficient power generation through burning fossil fuels, but will also minimise the emissions of $\mathrm{CO}_{2}$ into the atmosphere. In summary, utilisation of several different sources of renewable and clean energy in unison will be required to satiate the humongous power demands of the near future. 


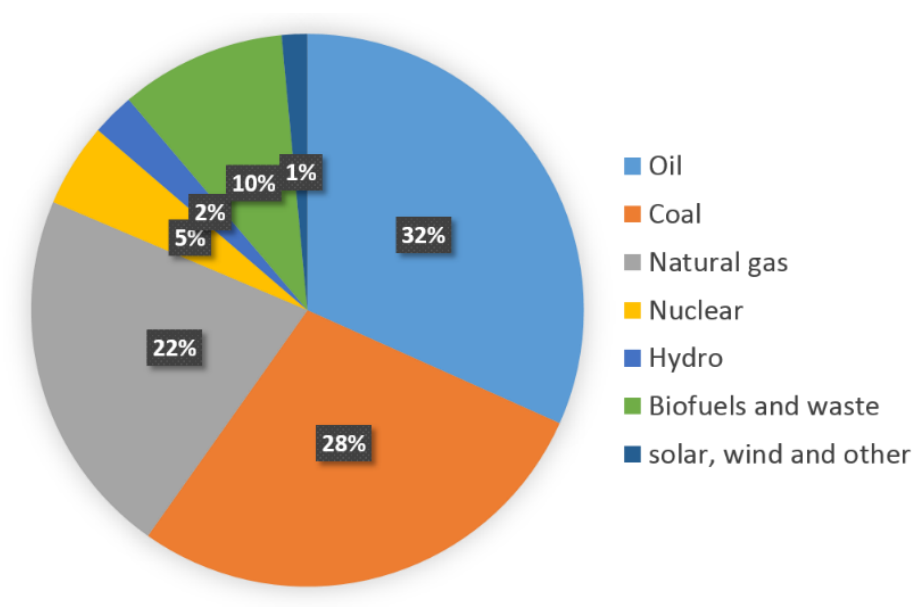

Figure 1.2 Spread of world total primary energy supply (TPES) in fuel in 2015. Data adapted from Ref. 3.

\subsubsection{Photovoltaics for the future}

Solar photovoltaics (PV) is a promising technology for large scale renewable energy harvesting. It is the fastest growing energy technology in the world, with annual installations having reached $50 \mathrm{GW}$, projected to increase in excess of $200 \mathrm{GW}$ by $2040,{ }^{4}$ with a net share of $1.7 \%$ of the global electricity production in 2017. Si wafer - based solar cells (Crystalline Silicon (c-Si), multi-crystalline Silicon (m-Si)) constitute 95\% of the global PV market, while other thin film technologies such as CdTe, CIGS and CzTs contribute the remaining $5 \%$ percent. ${ }^{5}$ Solar energy production is currently very cost competitive with hydroelectricity and slightly more expensive than the well-established wind technology in the United States of America. ${ }^{6}$

It is in this climate that the perovskite solar cell (PSC) has emerged as an exciting new candidate for photovoltaic technology. Historically, the first use of the perovskite in a solar cell was in 2009, as a solid-state absorber in a dye-sensitized solar cell (DSSC) configuration, with efficiency between 3-4\%, though with poor stability of the perovskite in the electrolyte. ${ }^{7}$ It was not until 2012 that it caught the attention of the photovoltaic community, with the formation of an all solid-state PSC with an efficiency of 9.7\%, with stability in excess of 500 hours. $^{8}$ 


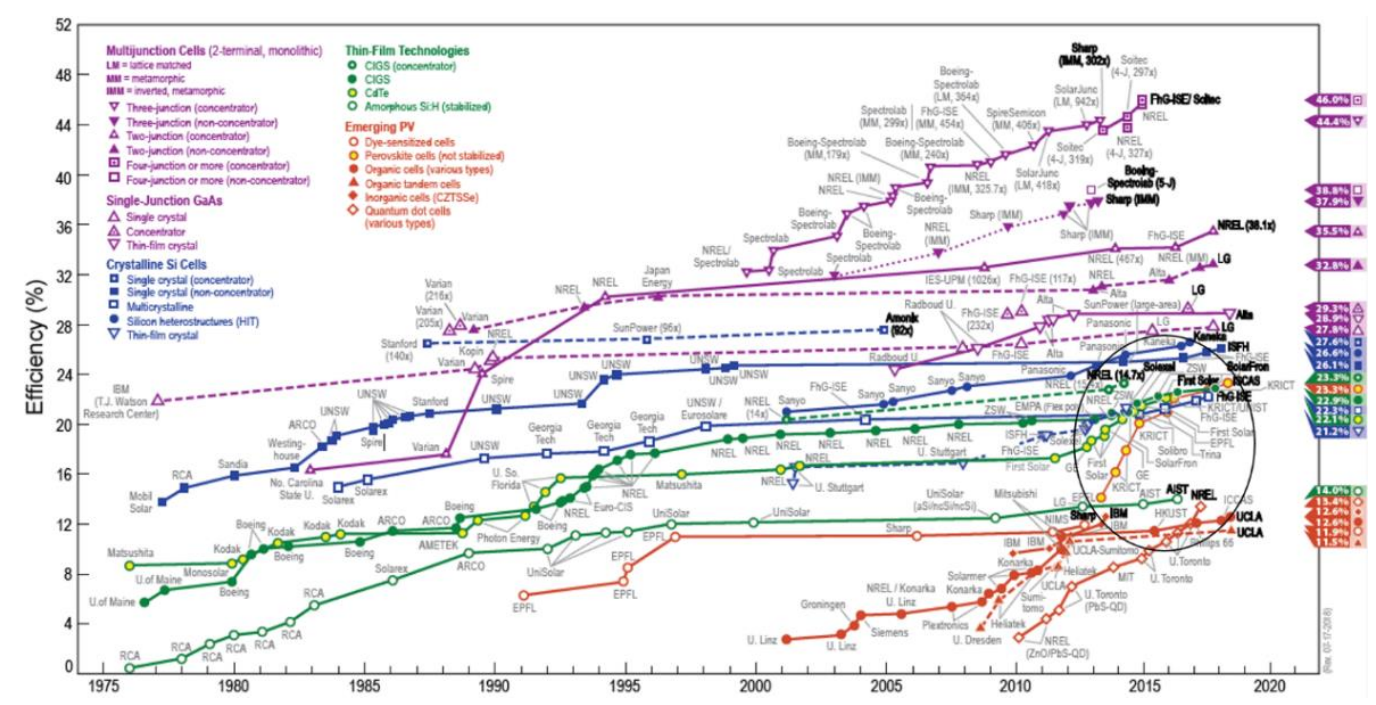

Figure 1.3 Evolution of efficiencies of several photovoltaic technologies. The circle highlights the efficiency rise of perovskite solar cells. Data obtained from National renewable energy laboratory (NREL), https://www.nrel.gov/pv/cell-efficiency.html.

Since then, there has been a rapid rise in the efficiency of these cells, with a record efficiency of $22.7 \%$ for a $\sim 0.1 \mathrm{~cm}^{2}$ cell. ${ }^{9}$ This is seen in Figure 1.3, where the efficiency evolution of c-Si and thin film technologies over 45 years has been matched by the perovskite in just 10 years. Furthermore, PSCs are easily prepared using low-cost solution processed methods such as spincoating for small device sizes typically in a lab, while screen printing and slot-dye coating are promising techniques for large scale production. ${ }^{10}$ The combination of low cost and high efficiency has led to the explosion of interest in these solar cells over the past few years.

\subsubsection{Perovskite Solar Cells}

This section will provide a general overview of the combination of unique physicochemical properties of the PSC that contributes to its excellent performance as a solar cell. This will be followed by an overview of the device operation properties of the PSC and current understanding of the physical mechanisms governing transport and recombination in these devices, in addition to presenting some open questions and validity of interpretations regarding the same.

\subsubsection{Device Structure}

The PSC works as an absorber layer sandwiched between two contacts that can inject and extract electrons and holes efficiently called the electron selective layer (ESL) and hole selective layer (HSL) respectively. Under illumination, the perovskite layer absorbs the incident radiation and generates electron-hole pairs, which are transported to the ESL and HSL respectively either due to an internal electrical field or by diffusion due to a gradient of concentration. These charges are then extracted by their respective selective 
contacts and through the outer circuit to yield the measured current.

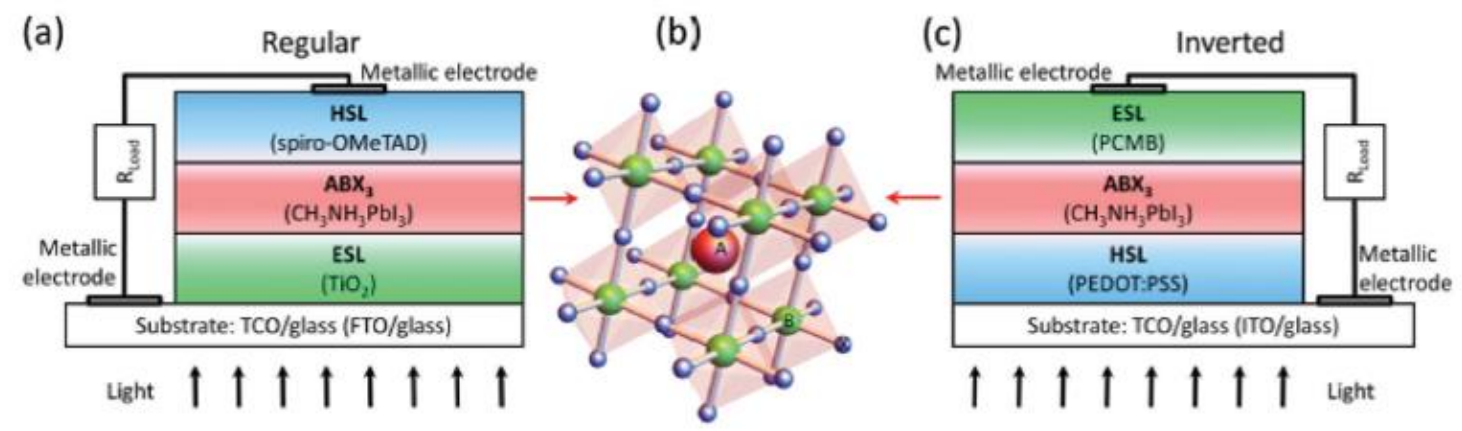

Figure 1.4 (a) Regular and (c) inverted device structures of the PSC, with the perovskite crystal structure shown in (b), red, green and blue spheres corresponding to A, B cations and $\mathrm{X}$ anion respectively. Adapted from Ref. 12, licensed by CC-BY-NC 4.0, http://creativecommons.org/licenses/by-nc/4.0.

The PSC is generally prepared in two different ways, ${ }^{11-12}$ termed regular and inverted structures, shown in Figure 1.4. The regular structure consists of a compact layer of $\mathrm{TiO}_{2}$ $\left(\mathrm{c}-\mathrm{TiO}_{2}\right)$ deposited on a glass substrate coated with a transparent conducting layer. Upon the $\mathrm{c}-\mathrm{TiO}_{2}$ layer, a nanostructured mesoporous $\mathrm{TiO}_{2}\left(\mathrm{~m}-\mathrm{TiO}_{2}\right)$ layer is deposited that extends into the absorber layer. The $\mathrm{c}-\mathrm{TiO}_{2}$ and $\mathrm{m}-\mathrm{TiO}_{2}$ layers together form the ESL in a regular structure. In some cases, the $\mathrm{m}-\mathrm{TiO}_{2}$ layer is omitted to yield the planar configuration. The absorber layer is sandwiched between the ESL and the HSL, which is a transparent organic layer, 2,2'(7,7')-tetrakis-(N,N-di- $p$-methyloxyphenyl-amine)9,9'spirobifluorene, commonly referred to as Spiro-OMeTAD. The inverted structure has the HSL, poly 4-styrenesulfonate, referred to as PEDOT:PSS, deposited on the glass substrate, followed by the perovskite absorber and then the ESL on top, an organic fullerene derivative [6,6]-phenyl-C-61-butyric acid methyl ester, commonly referred to as PCBM. 
(a)

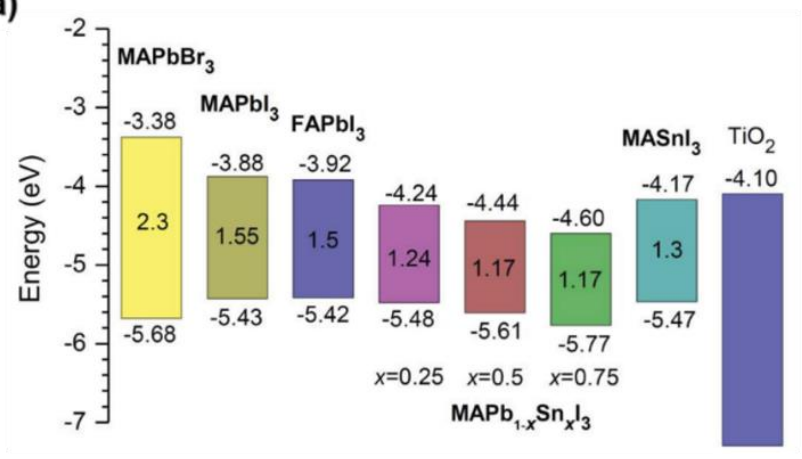

(b)

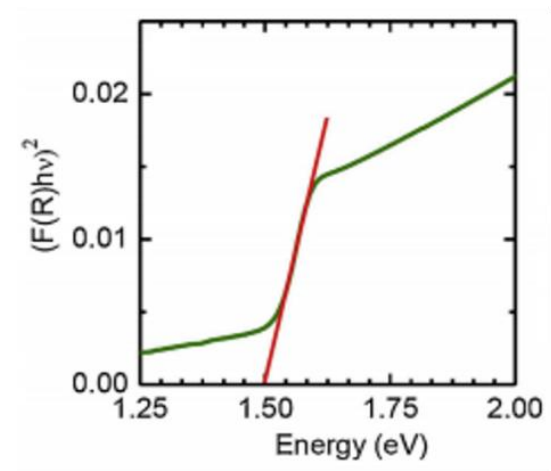

(c)

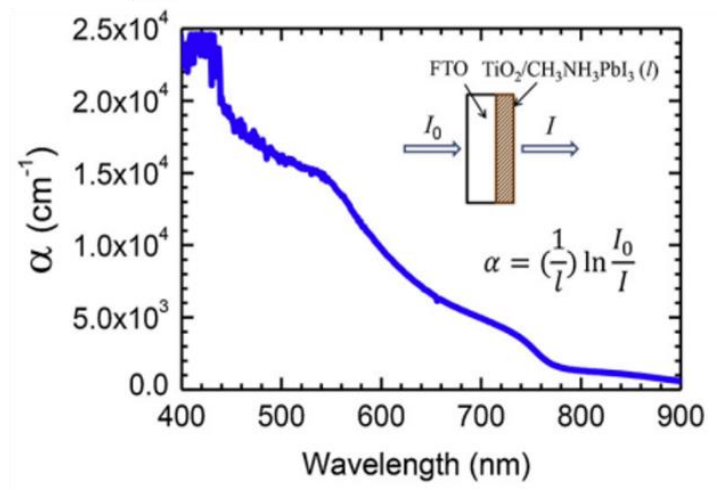

Figure 1.5 (a) Bandgaps of different types of perovskite absorbers and their respective valence and conduction band energy levels in comparison to the $\mathrm{TiO}_{2} \mathrm{ESL}$. (b) Bandgap of MAPI (red line intercept on the $\mathrm{x}$ axis) obtained from a Tauc plot and (c) absorption coefficient $\alpha$ obtained using the Beer-Lambert law from a transmittance measurement as shown in the inset, with $l$ corresponding to the thickness of the $\mathrm{TiO}_{2}$ nanoparticles sensitized by the perovskite absorber. (a) adapted from Ref. 20 with permission, Copyright 2014, WILEY VCH Verlag GmbH \& Co. KGaA, Weinheim. (b) and (c) adapted from Ref. 19, licensed by CC BY NC ND, http://creativecommons.org/licenses/by-nc-nd/4.0.

The perovskite absorber has the general formula $\mathrm{ABX}_{3}$, where $\mathrm{A}$ and $\mathrm{B}$ are cations and $\mathrm{X}$ is an anion. The perovskite crystallizes in a cubic or tetragonal structure (see Figure 1.4(b)), with the A cation being the largest and occupying the body center of the lattice. The B cations occupy the lattice points at the edges of the cube, forming an octahedron by coordinating with $6 \mathrm{X}$ anions. In PSCs, the choices for the $\mathrm{A}$ cation are Methylammonium $\left(\mathrm{CH}_{3} \mathrm{NH}_{3}\right)$, Formamidinium $\left(\mathrm{HC}\left(\mathrm{NH}_{2}\right)_{2}\right)$ and Caesium $(\mathrm{Cs})$. The B cation is usually Lead $(\mathrm{Pb})$ or Tin $(\mathrm{Sn})$, while the $\mathrm{X}$ halogen is either Iodine (I), Bromine $(\mathrm{Br})$ or Chlorine $(\mathrm{Cl})$. The most widely used perovskite is the hybrid organic-inorganic Methylammonium Lead Iodide $\left(\mathrm{CH}_{3} \mathrm{NH}_{3} \mathrm{PbI}_{3}\right)$, commonly and hereafter referred to as MAPI. MAPI is preferred as an absorber due to its near ideal bandgap between $1.5-1.61$ $\mathrm{eV}^{13-16}$ for solar energy production based on the Shockley-Queisser limit, ${ }^{17}$ sharp absorption edge ${ }^{18}$ and strong absorption in the visible part of the solar spectrum (see Figure 1.5(c)), which allows for absorption of the entire visible spectrum $(700 \mathrm{~nm}$ 
onwards) within $\sim 300 \mathrm{~nm} .{ }^{19}$ Furthermore, it is also relatively easy and cheap to produce on a laboratory scale using simple solution processed methods such as spincoating. Another attractive aspect of the perovskite is the ability to tune its bandgap, ${ }^{20}$ see Figure 1.5(a). For example, by adjusting the ratio of Iodine and Bromine, the bandgap of $\mathrm{CH}_{3} \mathrm{NH}_{3} \mathrm{~Pb}\left(\mathrm{I}_{1-\mathrm{x}} \mathrm{Br}_{\mathrm{x}}\right)_{3}$ perovskite was tuned from 1.6 to $2.3 \mathrm{eV} .{ }^{21} \mathrm{~A}$ similar strategy was used to tune the bandgap of $\mathrm{HC}\left(\mathrm{NH}_{2}\right)_{2} \mathrm{~Pb}_{\mathrm{y}} \mathrm{Br}_{3-\mathrm{y}}$ between 1.48 and $2.23 \mathrm{eV} .{ }^{15} \mathrm{Such}$ flexibility in the bandgap of the absorber can allow for the production of tandem cells consisting of perovskites that absorb different parts of the solar spectrum efficiently to move beyond the Shockley-Quiesser efficiency limit.
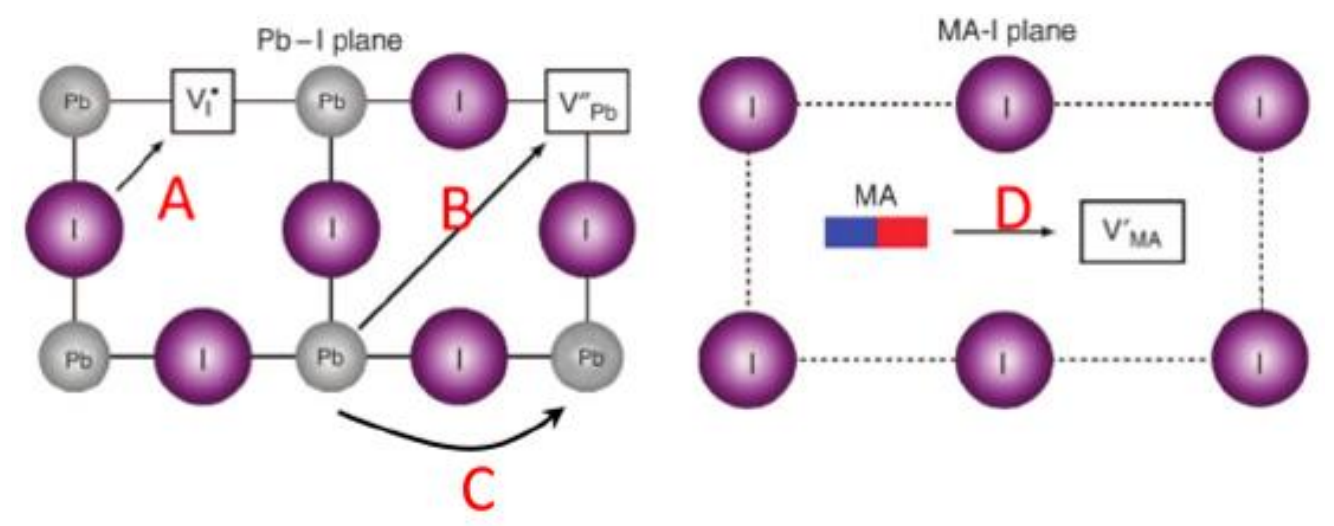

Figure 1.6 Different paths of ion migration via vacancies in a perovskite structure. Adapted from Ref. 23, licensed by CC BY 4.0, https://creativecommons.org/licenses/by/4.0.

In addition to its excellent optical properties, the perovskite is also a mixed electronicionic conductor. As is described in subsequent sections, there occurs a strong effect of migration of ions that is coupled to the device performance and the external parameters of light and voltage. Therefore, it is essential to understand which ions are migrating in the perovskite. In general, the displacement of an ion in a lattice is characterized by its diffusion coefficient $D$. This value depends on several aspects such as the nature of the crystal lattice, size and charge of the ion, density of interstitials/vacancies and ion hopping distance. Ionic motion is maximised in crystals with large densities of interstitials/vacancies, small ionic distances and smaller and less charged ions. ${ }^{22}$ In the case of the well-studied MAPI, the $\mathrm{I}^{-}$ion in the $\mathrm{PbI}_{6}{ }^{4-}$ octahedron has the smallest hopping distance to the nearest $\mathrm{I}^{-}$vacancy $(0.446 \mathrm{~nm})$ (process A in Figure 1.6), compared to $\mathrm{MA}^{+}$ and $\mathrm{Pb}^{2+}(0.628 \mathrm{~nm}) .{ }^{23}$ In fact, recent measurements of ionic and electrical conductivities in MAPI cells by varying $\mathrm{I}_{2}$ partial pressure,${ }^{24}$ in addition to wide-field photoluminescence (PL) imaging of MAPI under an applied electric field, ${ }^{25}$ provide strong evidence that the $\mathrm{I}^{-}$ion is the dominant mobile ionic species in MAPI, with an estimated diffusion coefficient of $D=10^{-8} \mathrm{~cm}^{2} \mathrm{~s}^{-1} \cdot{ }^{26-27}$ For the sake of simplicity, irrespective of which species is diffusing, the ionic movement is simply differentiated as anions and cations hereafter in this text. 


\subsubsection{Device Operation}

This section summarizes the different characteristics of PSCs that contribute to its high performance and evaluates the physical mechanisms underlying several of the unique observations reported for these cells. These include the prominent phenomenon of electrical hysteresis that depends on the history of the sample, unconventionally large capacitances and coupled resistances. A review of the transport and recombination properties of these devices is carried out, combined with the results of small perturbation techniques, culminating in a macroscopic device model that is currently favored in the community and its limitations.

\subsection{Hysteresis}

The operation of a solar cell is characterized by its current density (current per unit area) -voltage $(j-V)$ curve, which yields the parameters of efficiency, fill factor (FF) and maximum power point (MPP). Since the solar cell is a DC device, the measured points in a $j-V$ curve must correspond to steady state values. However, in practice, all $j-V$ curves are measured at a certain scan rate (V/s), however low it may be. PSCs are usually measured between scan rates of $50-200 \mathrm{mV} / \mathrm{s}$, and while there are several reports of very high efficiencies in the range of 15-20\%, it has been well documented that the PSCs suffer strongly from electrical hysteresis, which is a dependence of the $j-V$ curve values on the external parameters of the $j-V$ curve itself such as scan rate, scan direction, prebiasing voltage and light intensity. This leads to a problem of conflicting reports of PSC performance, which hinders differentiating between high and low performance cells and hence their development. Therefore, this section will summarize the various forms of hysteresis observed. 
(a)

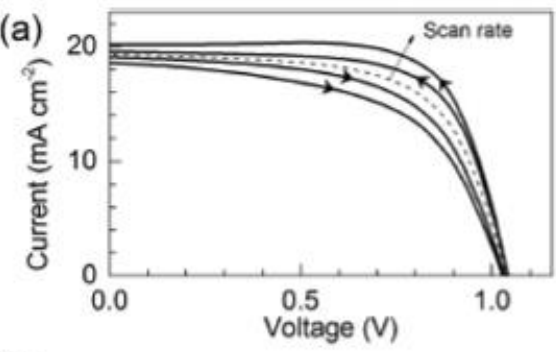

(c)

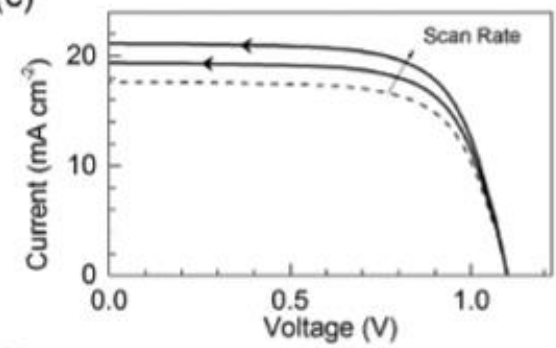

(e)

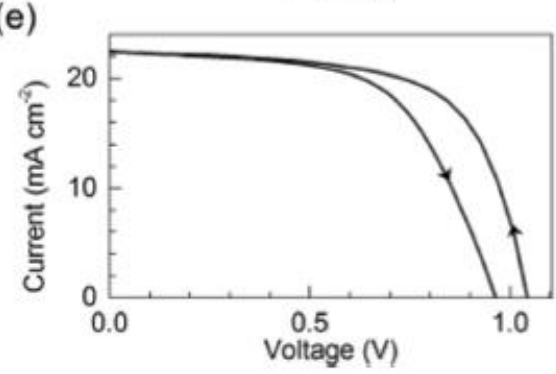

(b)

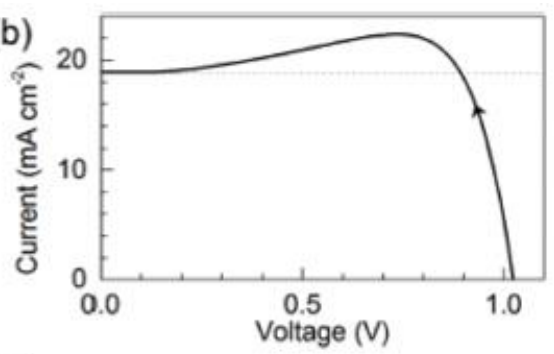

(d)

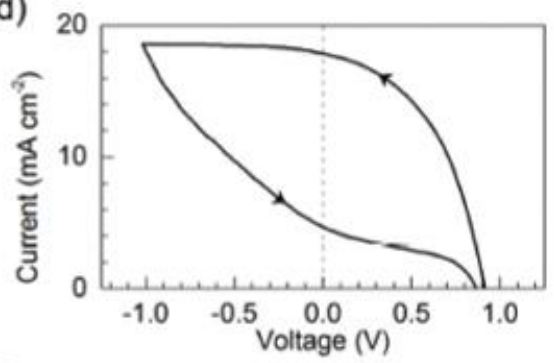

(f)

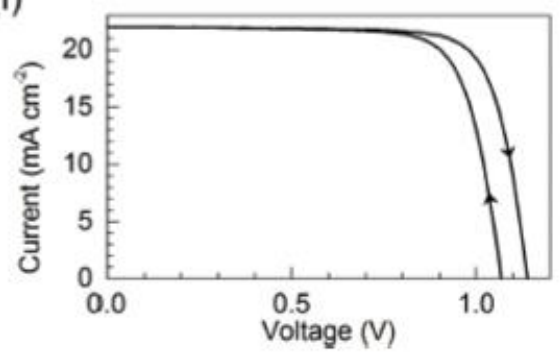

Figure 1.7 Summary of different types of electrical hysteresis observed in PSCs. The arrows indicate direction of the voltage sweep. Dashed lines indicate the steady state curve in a, c) and $j_{s c}$ and zero bias in b, d) respectively. Adapted from Ref. 11 with permission, Copyright 2018, WILEY VCH Verlag GmbH \& Co. KGaA, Weinheim.

Figure 1.7 shows the different types of electrical hysteresis commonly observed in PSCs. A voltage sweep from large forward bias (F) towards reverse bias (R) is termed the FR scan, and vice versa is termed the RF scan. These can be categorised as:

a) Capacitive hysteresis - This involves an enhancement in the current during a $j-V$ scan due to a capacitor-like discharge of current ${ }^{28-30}$ that is proportional to the scan rate and is symmetric around the steady state curve. This effect has been observed in dark conditions as well. ${ }^{31}$

b) 'Bump' close to $V_{\text {oc }}$ - A large discharge of current is observed upon the start of the FR scan which gradually diminishes. Always observed in the FR direction, influenced strongly by pre-poling at large forward bias. ${ }^{32-34}$

c) Apparent photocurrent enhancement in FR scan - This effect is similar to b) but extends over the entire range of voltage, yielding an apparent increase in the measured $j_{s c}$, while the $V_{o c}$ and forward bias resistance remain unchanged. ${ }^{32,34-37}$

d) Decay of photocurrent in RF scan - There occurs a strong reduction in the current during the RF scan, which also has a strong dependence on reverse bias prepoling. ${ }^{35,38}$ 
e) Apparent $V_{\text {oc }}$ shift for $R F$ scan - In this case, the current in the FR and RF scan are identical, except at large forward voltages, where it reduces sharply for the FR scan, yielding a smaller $V_{o c}$ for the FR scan compared to the RF scan. ${ }^{30,39-40}$ The combination of Figure 1.7(d) and 1.7(e) is the most commonly observed form of hysteresis in the PSCs and is termed 'normal hysteresis'.

f) Inverted Hysteresis - This is the opposite case of Figure 1.7(e), where the FR scan exceeds the RF scan at some point between SC and $V_{o c} \cdot{ }^{39-40}$ Usually, this is observed closer to $V_{o c}$, yielding a higher fill factor and $V_{o c}$ for the FR scan. However, in some cases, inverted hysteresis has been defined as an increase in only the $j_{s c}$ for an FR scan compared to the RF scan. ${ }^{41}$

As mentioned previously, the electrical hysteresis in PSCs is strongly dependent upon the conditions during and prior to the measurement. These parameters include voltage, light intensity, temperature and nature of contacts used. In order to understand the dependence of the PSC on these parameters, a summary of the hysteretic effects of each of these parameters is provided in the following sections.

\subsection{Effect of voltage preconditioning}

(a)

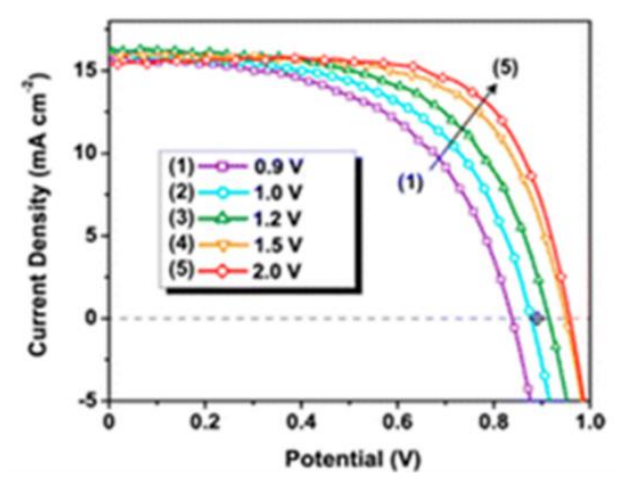

(b)

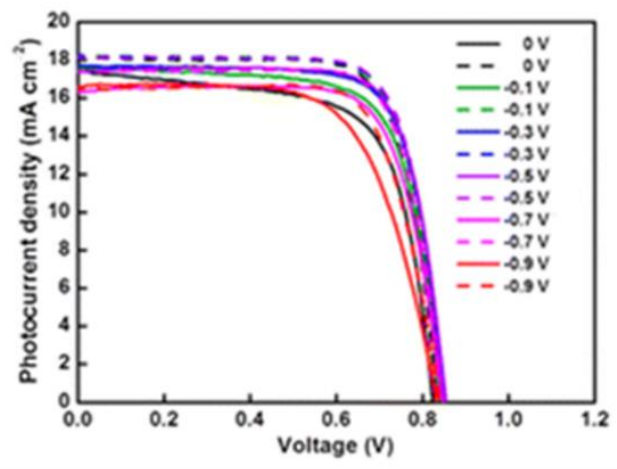

(c)

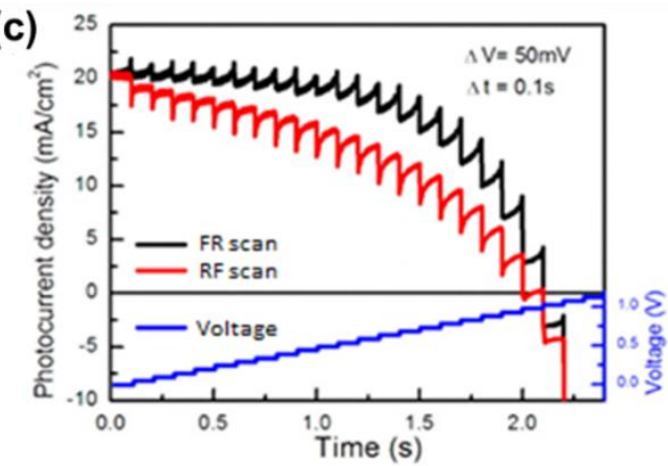

Figure 1.8 Effect of (a) forward bias and (b) reverse bias pre-poling on the $j-V$ curve of a PSC. Dashed lines and solid lines indicate FR and RF scans respectively. (c) Evolution of current versus time for stepped voltage scans, with step size, delay time and scan direction indicated in insets. The black diamond in (a) indicates stabilized $V_{o c}$ when holding the device under 1 sun illumination for 60 seconds. (a) Adapted from Ref. 37 
with permission, Copyright 2015, American Chemical Society. (b) adapted from Ref. 43, Copyright 2014, American Chemical Society. (c) adapted from Ref. 29, Copyright 2015, American Chemical Society.

Voltage pre-conditioning has a strong effect on the obtained $j-V$ curve for a PSC. This is summarised in Figure 1.8. The application of a large forward bias for an extended period of time is well known to improve the $V_{o c}$ and FF of the $j-V$ curve during a FR $\operatorname{scan}^{32,35,37,42}$ as shown in Figure 1.8(a), which can also result in a 'bump' as described in Figure 1.7(b). In the case of reverse bias pre-poling, the RF scan shows a reduced $j_{s c}$ compared to the FR scan. For large reverse biases, there occurs a large drop in $j_{s c}$ and FF for the RF scan and in some cases, even the $V_{o c}$ is severely reduced. ${ }^{35,37,43}$ These effects are also observed from stepped voltage scans, which involves measuring the current upon application of a voltage step from a given condition and maintaining the voltage for a given time period, termed the delay time, before application of the next voltage step. In these measurements (Figure 1.8(c)), the capacitive discharge is highest close to $V_{o c}$, with a drop-off on either side. ${ }^{29,32,44}$ Furthermore, the decay lifetimes of these capacitive transients has been observed to be in the order of a few hundred milliseconds to a few seconds. ${ }^{29,32-33}$

\subsection{Effect of light and temperature}

(a)

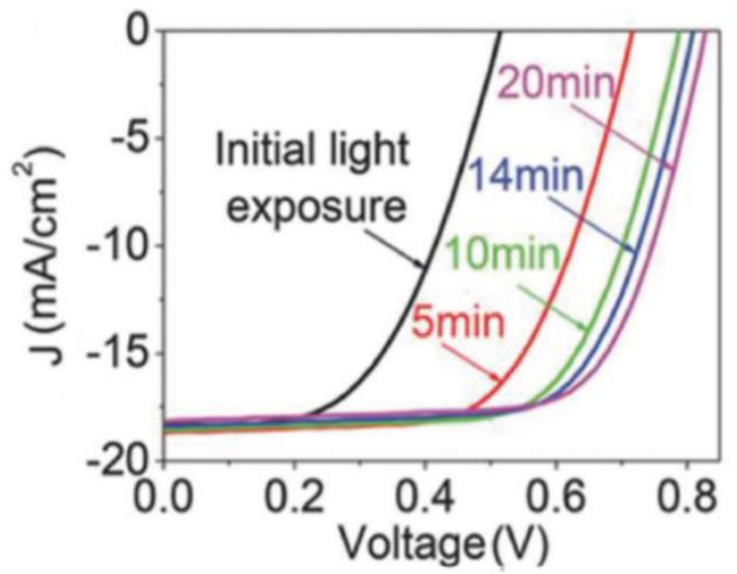

(b)

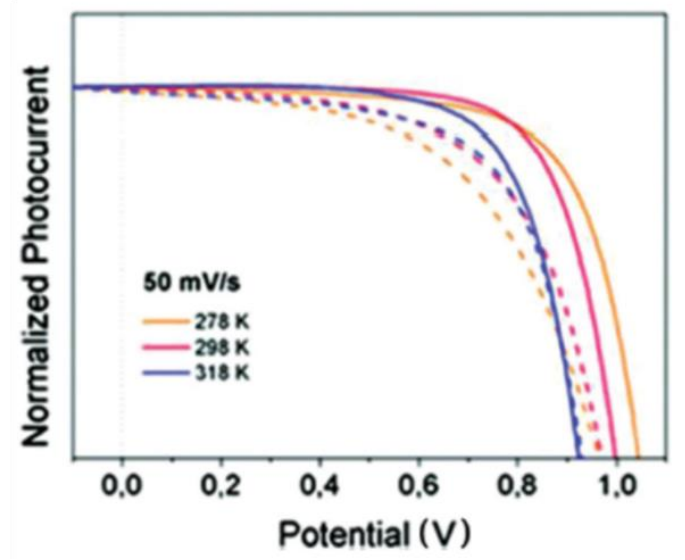

Figure 1.9 Effect of (a) light soaking and (b) temperature on the $j-V$ curves of the MAPI solar cell. Solid lines and dashed lines indicate FR and RF scans respectively. (a) Adapted from Ref. 45 with permission, Copyright 2018, WILEY VCH Verlag GmbH \& Co. KGaA, Weinheim. (b) Adapted from Ref. 48 with permission, Copyright 2016, Royal Society of Chemistry.

The effect of light soaking a PSC is shown in Figure 1.9(a). In general, light soaking improves the fill factor and $V_{o c}$ of the PSC, ${ }^{32,45-46}$ while the $j_{S C}$ has not been seen to evolve 
significantly. Light soaking does not have an effect on the shape and scaling of the type of hysteresis observed. ${ }^{35}$ The effects of light soaking have been found to be reversible, with the PSCs regaining almost their entire pre-soaking performance upon extended dark storage. ${ }^{47}$ Temperature also has a strong effect on the $j-V$ curve of a PSC, as seen in Figure 1.9(b). Hysteresis has been observed to increase at temperatures below room temperature, ${ }^{33}$, 48-49 including PSCs that are apparently 'hysteresis-free' at room temperature. ${ }^{50}$ As discussed previously, the density of mobile ions in the perovskite can alter local electrical fields, affecting charge transport, recombination and extraction. Therefore, the effect of light soaking and temperature is generally attributed to a change in the density of mobile ionic carriers in the absorber ${ }^{51}$ and their drift due to thermal activation. These processes are further complicated by ionic reactivity at the ESL/perovskite and HSL/perovskite layers, ${ }^{52-53}$ which makes development of a generalised model to explain these effects very difficult.

\subsection{Effect of Contacts}

(a)

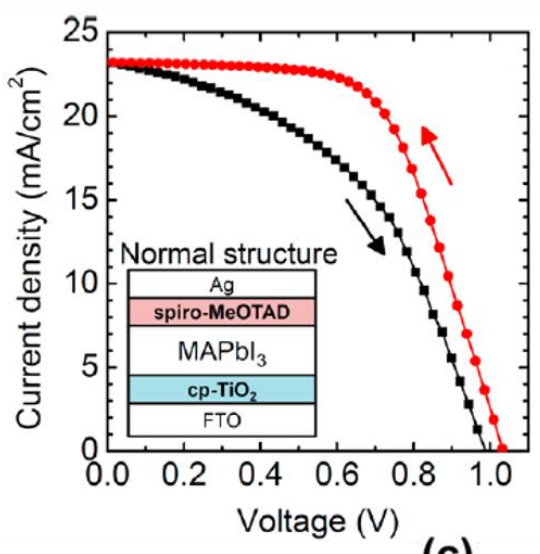

(b)

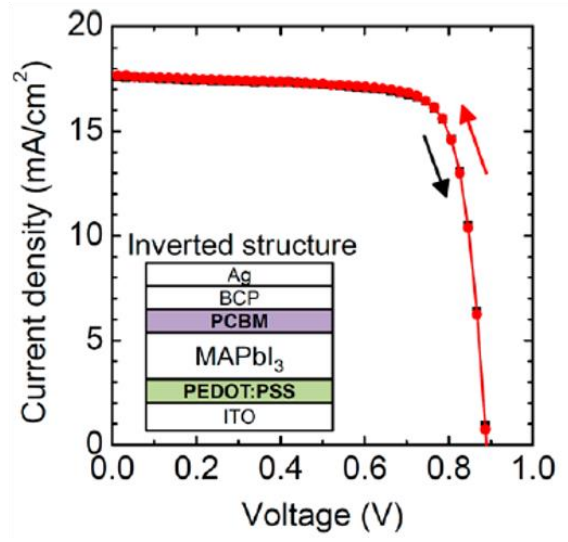

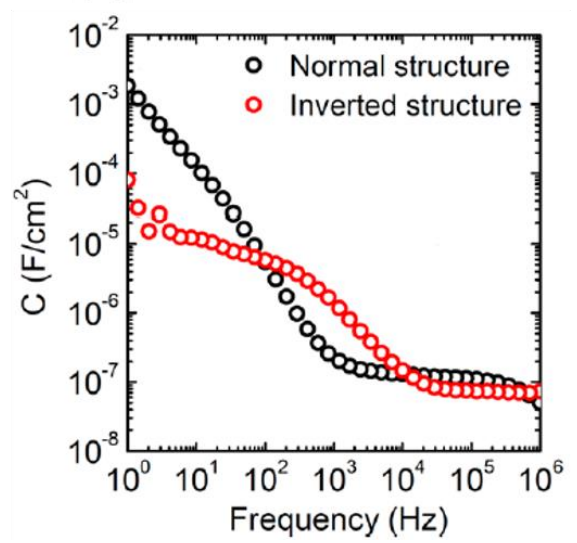

Figure 1.10 Effect of (a) $\mathrm{TiO}_{2}$ ESL (normal structure) and (b) PCBM ESL (inverted structure) on the hysteretic behaviour of MAPI solar cells. (c) Capacitance versus frequency for normal and inverted structures obtained under 1 sun illumination at short circuit conditions. Adapted from Ref. 57 with permission, Copyright 2015, American Chemical Society. 
The selective contacts in the perovskite solar cell appear to play a very important role in its operation. This is particularly prominent with respect to the ESL, where the replacement of $\mathrm{TiO}_{2}$ with PCBM, either as a compact interfacial layer ${ }^{54}$ or mixed in to the absorber in a bulk heterojunction configuration, ${ }^{55}$ shows a marked reduction in the hysteresis of the $j-V$ curve, shown in Figure 1.10(a) and 1.10(b). This effect is directly observed in the inverted configuration perovskite solar cells, whose ESL and HSL are PCBM and PEDOT:PSS respectively, show very little hysteresis in general. Capacitance measurements show a very large capacitance of the order of milliFarads $(\mathrm{mF})$ for $\mathrm{TiO}_{2}$ based cells at both $\mathrm{OC}$ and SC conditions under illumination, ${ }^{56}$ which is reduced by several orders upon replacement with PCBM, as shown in Figure 1.10(c) ${ }^{57}$ This indicates the storage of a large density of charge which is strongly affected by the perovskite/selective layer interfaces. This is discussed in detail in Section 1.2.3.3.1. Recently, the alignment of the valence bands of the perovskite and the HSL have been shown to control the hysteretic behaviour in Fullerene-based MAPI solar cells. ${ }^{58}$

In addition to hysteretic behaviour and charge accumulation, the selective contacts can also determine the built-in potential $V_{b i}$ and its distribution within the device based on the doping density of the absorber layer, hence having a strong effect on the obtained $V_{o c}{ }^{59}$ Several experiments have shown that MAPI devices without an ESL is able to perform efficiently and generate similar $V_{o c}$ values compared to a complete device. ${ }^{60}$ This indicates that the potential drop during contact equilibration is likely occurring mainly at the interfaces and hence, the perovskite absorber itself is efficient at separating and transporting photogenerated charges.

\subsection{Recombination - Fast and slow timescales}

Recombination is a fundamental process in the solar cell, where an electron that has been excited to the conduction band (CB) from the valence band (VB) by absorption of a photon, returns to the valence band. This recombination can occur radiatively, by the emission of a photon whose energy is equal to the energy difference between the $\mathrm{CB}$ and $\mathrm{VB}$, or non-radiatively through mid-gap states, where the energy is lost in the form of lattice vibrations or phonons. Due to the principle of detailed balance, which balances opposite fluxes at equilibrium, the $V_{o c}$ attainable by a solar cell is proportional to the logarithm of the external quantum efficiency of the solar cell operated as a light-emitting diode (LED), called EQE ${ }_{\text {LED. }}{ }^{61}$ This maximum $V_{o c}$ attainable, termed the radiative $V_{o c}$, is smaller than the bandgap and is governed by the radiative recombination constant or reverse saturation current density, that is determined mainly by the sharpness of the absorption spectra at its onset wavelengths. ${ }^{62}$ Therefore, it is of crucial importance to minimise non-radiative recombination via defects, traps and tail states within the bandgap in the bulk or at interfaces and promote out-coupling of emitted photons from radiatively recombining electronic carriers. The amount of radiative recombination per unit volume per second can be expressed by

$$
U=B_{\text {rad }}\left(n p-n_{i}^{2}\right)
$$


where $B_{\text {rad }}$ is the radiative recombination constant (volume second), $n$ and $p$ are the density of free electrons and holes respectively (per unit volume per second) and $n_{i}^{2}=$ $n_{0} p_{0}$, where $n_{0}$ and $p_{0}$ are the equilibrium free electron and hole densities (per unit volume per second) respectively. This is termed bimolecular recombination because the recombination depends on the densities of both free electrons and holes. In the case of a doped semiconductor, we have monomolecular recombination of the minority carriers described by

$$
U=B_{\text {rad }} N_{A}\left(n-n_{0}\right)
$$

where $N_{A}$ is the density of acceptors in a p-type material. Non-radiative recombination via traps or mid-gap states is also called Shockley-Read-Hall (SRH) recombination, given by $^{63}$

$$
U_{S R H}=\frac{p n-p_{1} n_{1}}{\tau_{p}\left(n+n_{1}\right)+\tau_{n}\left(p+p_{1}\right)}
$$

where $p_{1}$ and $n_{1}$ are the densities of electrons and holes when the Fermi level coincides with the energy level of the trap and $\tau_{p}$ and $\tau_{n}$ are the lifetimes of holes and electrons in a highly doped $\mathrm{n}$ and $\mathrm{p}$-type semiconductor respectively.

Non-radiative recombination can also occur at the blocking interface of a selective contact, for example, excess electrons at the perovskite/HSL interface or holes at the perovskite/ETL interface. This is stated in terms of a surface recombination velocity $S_{n}$

$$
j_{n}=S_{n}\left(n-n_{0}\right)
$$

where $j_{n}$ is the current density of recombining minority carriers. A contact can be blocking or absorbing when $S_{n} \rightarrow 0$ and $S_{n} \rightarrow \infty$ respectively. ${ }^{62}$

Both radiative and non-radiative recombination can be probed by several techniques in order to understand the nature and localisation of recombination processes. One such technique that has been applied to MAPI solar cells is transient photoluminescence (PL) decay, which involves the application of a monochromatic pulse of photons and subsequently measuring the decay of the luminescence. Using the continuity equation

$$
\frac{d n}{d t}=-U
$$

and the fact that the number density of emitted photons is given by the recombination current

$$
j_{r e c}=q L U
$$

where $\mathrm{L}$ is the thickness of the sample, it becomes possible to distinguish between monomolecular and bimolecular recombination using Equations 1 and 2 and appropriate boundary conditions. 
(a)

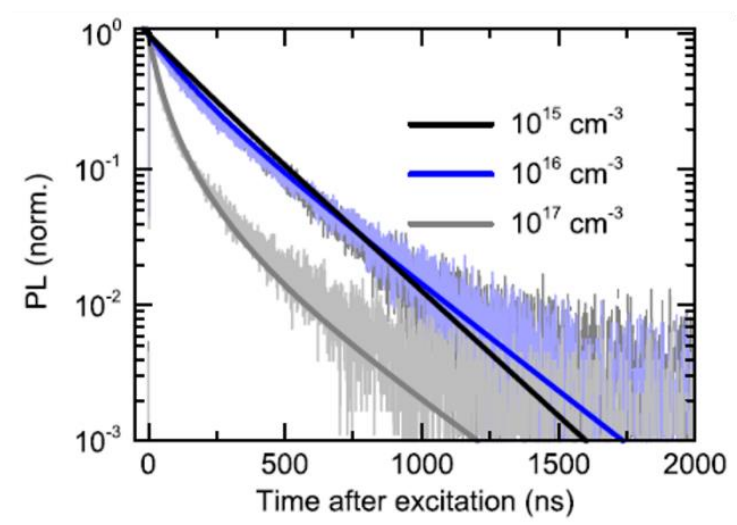

(b)

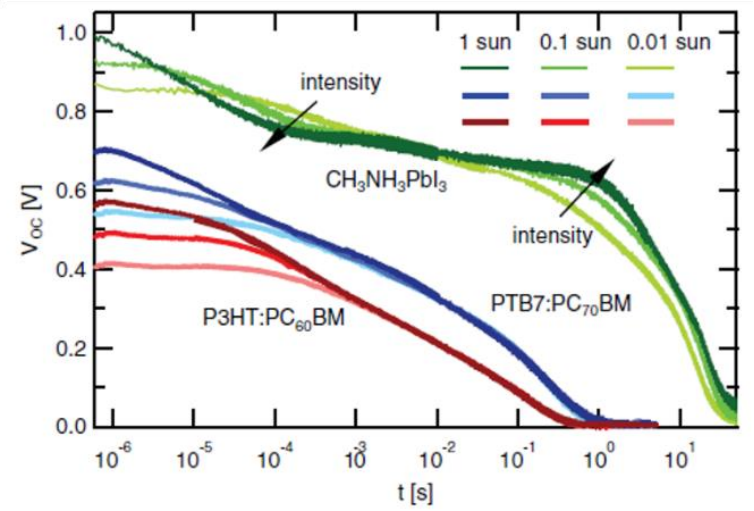

Figure 1.11 (a) Transient PL decays at $780 \mathrm{~nm}$ for different photoexcited carrier densities as shown in the legend for MAPI solar cells. Adapted from Ref. 64 with permission, Copyright 2014, American Physical Society. (b) Evolution of $V_{o c}$ versus time for MAPI (green), PTB7:PC $70 \mathrm{BM}$ (blue) and P3HT: $\mathrm{PC}_{60} \mathrm{BM}$ (red) solar cells for different steady state background illumination intensities as shown in the legend. Adapted from Ref. 66, licensed by CC BY 4.0, https://creativecommons.org/licenses/by/4.0/.

Figure 1.11(a) shows the strong dependence of the luminescence of MAPI solar cells on the light intensity. While monomolecular recombination is observed for low fluences, there occurs a transition to a power law decay at higher fluences, indicative of higher orders of recombination. This has been interpreted based on the filling of traps, where a background density of free, photodoped holes due to trapped charges dominates the recombination at low fluences, added to the recombination of photogenerated carriers at high fluences. ${ }^{64}$ In addition to the intensity-dependent fast recombination, perovskite solar cells also show unique recombination characteristics at longer timescales. These are observed from $V_{o c}$ decay measurements, which allows monitoring the evolution of the minority carrier Fermi level and can hence be used to quantify recombination lifetimes and charge densities. ${ }^{65}$ Figure 1.11(b) shows the $V_{o c}$ decay of a MAPI solar cell in comparison to two different organic solar cells under different initial steady state illumination intensities as shown in the legend. ${ }^{66}$ Unlike its organic counterparts, the MAPI solar cell shows a very slow decay between 1-100 milliseconds, which accelerates slightly at longer timescales. Even after several seconds, there is a non-zero $V_{o c}$ that exists, indicating extremely long lifetimes for the electronic carriers. This was also observed in MAPI cells without a HSL and attributed to the accumulation of majority holes and Iodide vacancies at the perovskite/ $\mathrm{TiO}_{2}$ interface under steady state illumination. ${ }^{67}$ The slow drift of these ions upon removal of the light is deeply connected to the recombination of this charge at the interface, yielding the very slow recombination timescales observed. Furthermore, these timescales are comparable to the voltage sweep times in a $j-V$ curve and hence are a strong indication of the processes that control the hysteresis patterns of 
Figure 1.7 .

\subsection{Transport}

Photogeneration creates bound pairs of electrons and holes in the $\mathrm{CB}$ and VB respectively, called excitons. These excitons have a binding energy associated with them that needs to be provided to break their interactions and generate free electrons and holes, which subsequently need to be transported to their respective contacts. This transport can occur due to an electrical field within the absorber that tilts the bands, or by diffusion due to a concentration gradient. ${ }^{62}$ In general, the electron and hole currents are given by

$$
\begin{aligned}
& j_{n}=q n \mu_{n} E+q D_{n} \frac{\partial n}{\partial x} \\
& j_{p}=q p \mu_{p} E-q D_{p} \frac{\partial p}{\partial x}
\end{aligned}
$$

where $q$ is the electronic charge, $n$ and $p$ are the densities of electrons and holes respectively, $E$ is the electrical field and $\mu_{n(p)}, D_{n(p)}$ are the conductivity and diffusion coefficient of electrons (holes). We note that $E, n$ and $p$ are all functions of position $x$ along the length of the semiconductor and that the mobility and diffusion coefficient are related by the Einstein equation

$$
D_{n(p)}=\mu_{n(p)}\left(\frac{k_{B} T}{q}\right)
$$

These currents can also be expressed in terms of the electron Fermi level $E_{F n}$ and hole Fermi level $E_{F p}$ as

$$
\begin{aligned}
& j_{n}=n \mu_{n} \frac{\partial E_{F n}}{\partial x} \\
& j_{p}=p \mu_{p} \frac{\partial E_{F p}}{\partial x}
\end{aligned}
$$

An important parameter that is used to characterize transport in a solar cell is the diffusion length, which is the distance a minority electron or hole travels before it recombines. The diffusion length is given by

$$
L_{D}=\sqrt{D \tau_{\text {rec }}}
$$

where $\tau_{\text {rec }}$ is the characteristic timescale (lifetime) of recombination of the minority carrier. $\tau_{r e c}$ is generally a function of the minority carrier Fermi level, being a constant only for first-order recombination. ${ }^{65}$

As mentioned previously, the first process of photovoltaic action upon photogeneration involves the breaking of excitons, whose difficulty is characterized by their binding energy. In the case of perovskites, this binding energy at room temperature $(298 \mathrm{~K})$ is of the order of a few millielectronvolts $(\mathrm{meV}),{ }^{68-69}$ lower than the average thermal energy per particle $k_{B} T(26 \mathrm{meV})$. This indicates that photogeneration in perovskites essentially yields free carriers. These free carriers then travel a distance equal to their diffusion length before recombining, indicating that the collecting contact must be within a diffusion length's distance of photocarrier generation. Several reports indicate very large diffusion lengths for both electrons and holes in MAPI solar cells. From transient 
photoluminescence measurements on glass/MAPI cells with one-sided selective and blocking contacts, the diffusion lengths of both electrons and holes in $\mathrm{MAPI}_{3-\mathrm{x}} \mathrm{Cl}_{\mathrm{x}}$ solar cells was found to be in excess of $1 \mu \mathrm{m}, 10$ times larger than that in MAPI solar cells. ${ }^{70}$ Diffusion lengths in the order of $\mu \mathrm{m}$ have also been measured for Bromide-based PSCs. ${ }^{71-}$ ${ }^{72}$ Similarly, large mobility values between $9-164 \mathrm{~cm}^{2} \mathrm{~V}^{-1} \mathrm{~s}^{-1}$ have been estimated ${ }^{73-76}$ for PSCs. Therefore, it appears that the perovskite is able to efficiently separate photogenerated charges to their respective selective contacts, and the role of an internal electric field must therefore be relatively inconsequential.

\subsubsection{Insights from small perturbation methods}

The $j-V$ curve of a solar cell does not provide much information regarding the underlying mechanisms driving transport, recombination and charge storage. Therefore, measuring the response of a solar cell to a small perturbation of different inputs such as voltage or light in the frequency domain is a useful technique to separate internal mechanisms occurring on different timescales, allowing to identify capacitive mechanisms and separate internal resistances. This understanding is summarised in the form of an equivalent circuit (EC), which represents the underlying physical processes in terms of passive electrical elements such as resistors, capacitors, inductors and current and voltage sources. Two prominent small perturbation techniques that have been used to study PSCs are impedance spectroscopy (IS) and intensity modulated photocurrent spectroscopy (IMPS). The information obtained from these two techniques is summarised in the following sections.

\subsection{Capacitances and Resistances}

IS has traditionally been a useful tool to study two important aspects of a solar cell, namely its internal resistances and capacitances. These resistances represent physical mechanisms of voltage drops within the solar cell, such as recombination and poor conductivity, while capacitances can provide information on doping density and density of states of the semiconductor and band bending at interfaces. The IS transfer function and definitions are provided in Section 2.1. 


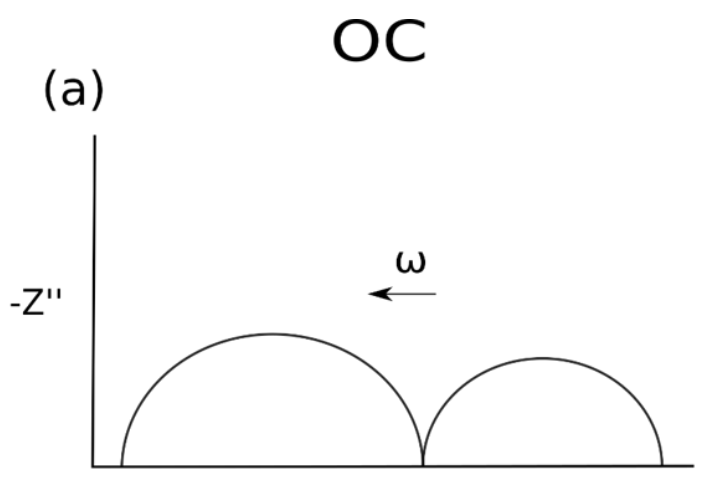

$Z^{\prime}$

(b)

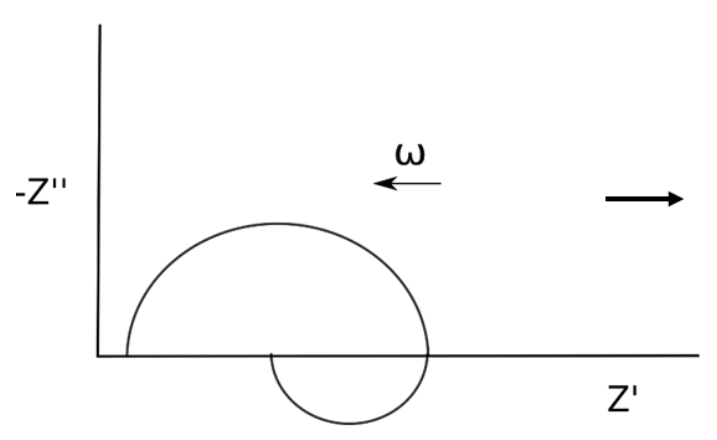

(c)

SC

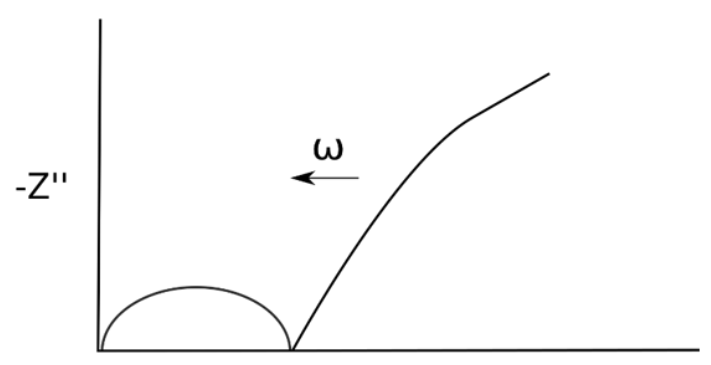

$Z^{\prime}$

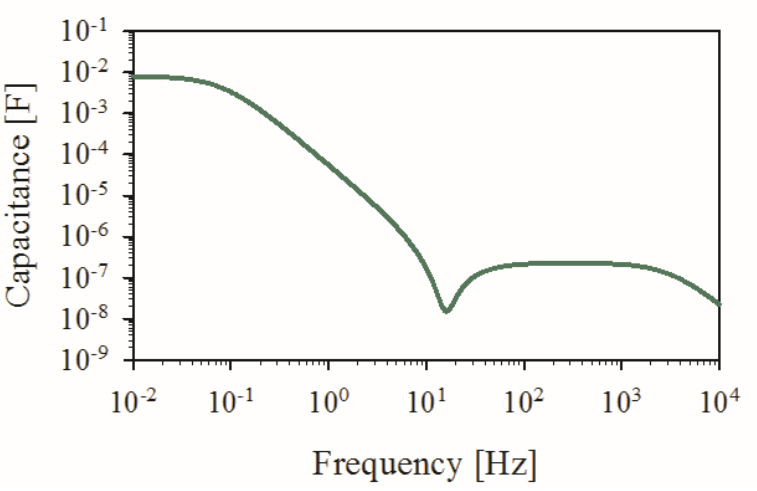

Figure 1.12 Typical IS Z-plane plots observed at (a), (b) open circuit conditions and (c) short circuit conditions under illumination.

Perovskite solar cells suffer from a large variability in the observed IS Z-plane plots, ${ }^{77}$ related in turn to a large inherent variability in materials and performance so far, which prevents identifying a consistent spectrum for interpretation. However, the most frequently observed Z-plane plots are shown in Figure 1.12. At OC conditions under 1 sun illumination, MAPI solar cells show two arcs in the upper quadrant, Figure 1.12(a). These arcs are seen to evolve in a correlated manner with voltage and illumination intensity, ${ }^{56}$ indicating a coupling between the resistances and/or capacitances. There also occurs exotic spectra, where the response moves to the lower quadrant at low frequencies, indicating the transition to a negative capacitance, as shown in Figure 1.12(b). ${ }^{28,78-79}$ While there are different ways to generate a negative capacitance such as the use of an inductor in the $\mathrm{EC},{ }^{80}$ a variable series resistance that depends on the conductivity, ${ }^{81}$ a general consensus has not yet been achieved regarding the mechanism of this effect in PSCs. Another interesting observation involves the formation of a loop in the upper quadrant, ${ }^{80,82}$ which also appears to be related to an inductive effect. At SC conditions, two arcs are observed (Figure 1.12(c)), with the low frequency (LF) arc width being very large due to the large resistances at SC, while the high frequency (HF) arc width is between $40-100 \Omega \cdot \mathrm{cm}^{2} .{ }^{56}$ Both these arcs show a diminishing trend with increasing light 
intensity.

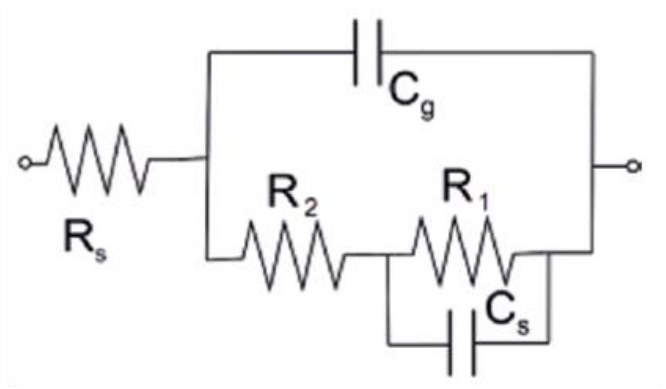

(a)

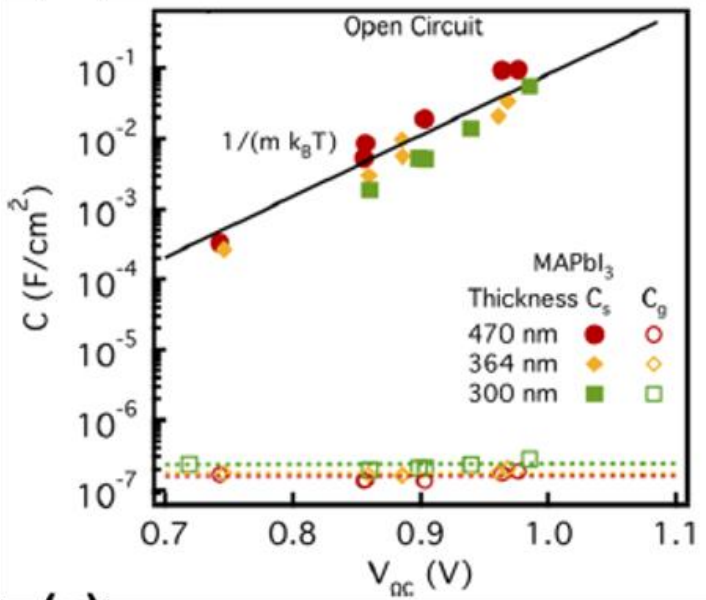

(c)

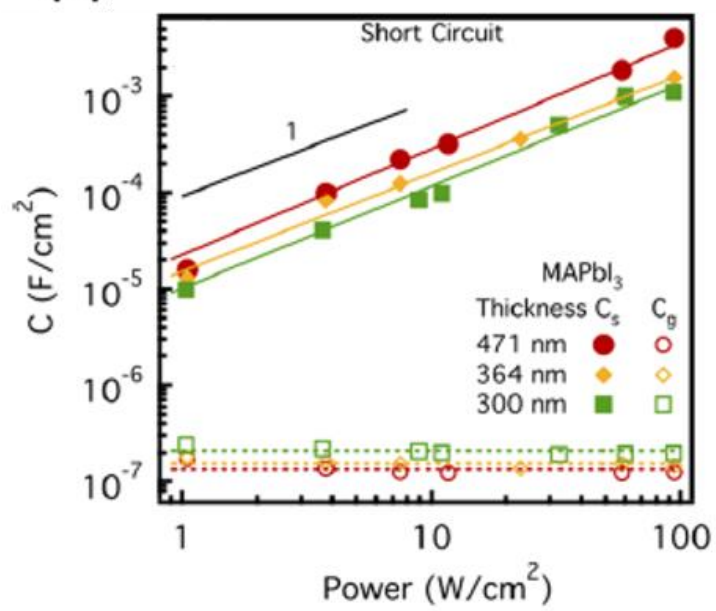

(b)

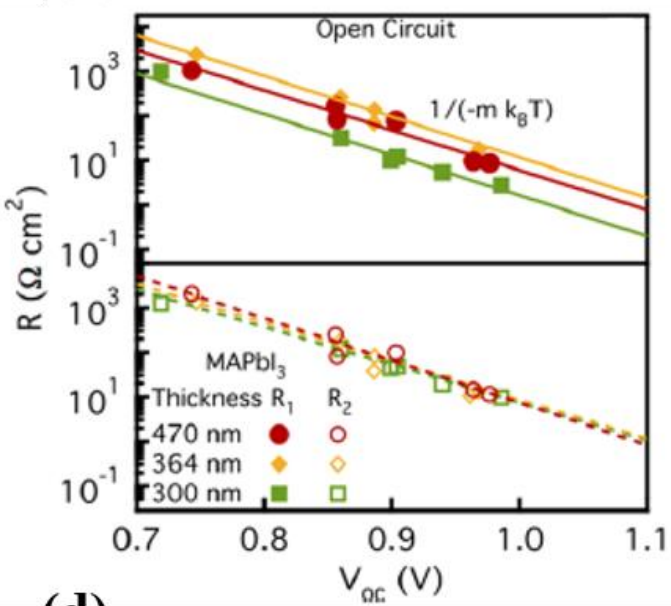

(d)

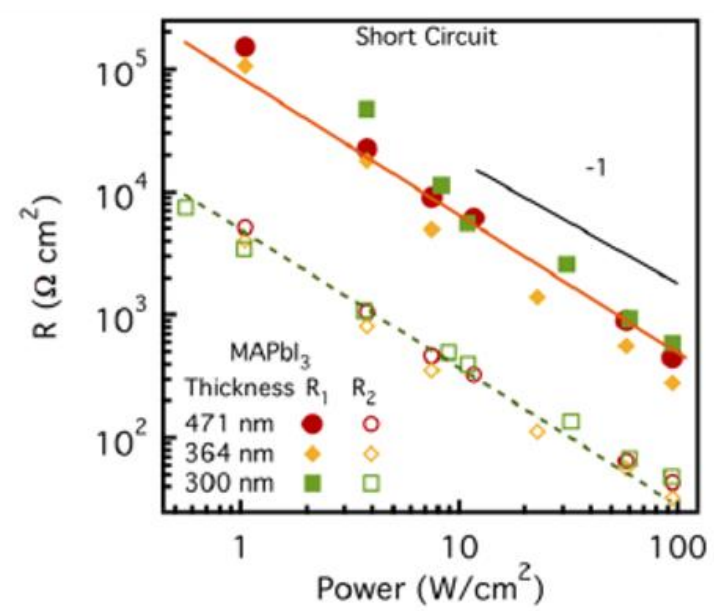

Figure 1.13 Equivalent circuit (top panel) used to fit the IS data of MAPI solar cells of different thicknesses as shown in legend. Evolution of (a), (c) capacitances and (b), (d) resistances at $\mathrm{OC}$ and $\mathrm{SC}$ conditions respectively. Black lines with values indicate slopes. Adapted from Ref. 56 with permission, Copyright 2016, American Chemical Society.

The most reliable EC used to fit the IS data of perovskite solar cells and the corresponding extracted parameters are shown in Figure 1.13. At both OC and SC conditions (Figure 1.13(a) and 1.13(c) respectively), a LF capacitance that evolves exponentially with both 
voltage and light intensity is observed, reaching values as high as a few milliFarads $(\mathrm{mF})$. Such a large value of the capacitance indicates that it is not the chemical capacitance, whose value is limited by the density of states of the absorber. Therefore, it was ascribed to electrode polarization, ${ }^{31}$ due to the accumulation of positively charged ions at the perovskite/ESL interface, akin to a Helmholtz double layer capacitance at a semiconductor/electrolyte interface. However, the huge capacitance values indicate either an unrealistically large dielectric constant or an extremely small ionic radius, which is the minimum separation between the two plates of the Helmholtz capacitor. The Helmholtz capacitance per unit area is given by

$$
C_{H}=\frac{\varepsilon \varepsilon_{0}}{l}
$$

where $\varepsilon$ is the dielectric constant of the perovskite, $\varepsilon_{0}$ is the permittivity of vacuum and $l$ is the separation between the plates of the capacitor. Another possible explanation is the existence of a diffuse layer of ions, whose capacitance is known to evolve exponentially with voltage. ${ }^{83}$ However, this would indicate a large variation in the density of ions with light intensity and furthermore, the diffuse layer capacitance is in series with the Helmholtz capacitance and hence, the net capacitance is dominated by the Helmholtz capacitance. Based on this reasoning, in addition to the observation of an unbalanced distribution of charge at either selective contact under illumination, ${ }^{84}$ the formation of an accumulation layer at OC conditions at the perovskite/ESL was proposed, shown in Figure 1.14. ${ }^{85}$ Briefly, assuming a p-type perovskite, at large forward biases or light intensities, the large amount of negative electronic charge at the ESL brings a net density of positively charged ions to the ESL/perovskite interface, which results in a net upward band bending on the perovskite side that crosses the hole Fermi level and increases the concentration of holes. Therefore, an accumulation of majority carriers (holes), facilitated by the positively charged ions, occurs at the ESL/perovskite interface. The capacitance of an accumulation layer is given by ${ }^{86}$

$$
C_{a c c}=\mathrm{q}\left(\frac{p_{b} \varepsilon_{r} \varepsilon_{0}}{2 k_{B} T}\right)^{1 / 2} e^{\left(q V / 2 k_{B} T\right)}
$$

where $p_{b}$ is the bulk majority carrier (hole) concentration, $\varepsilon_{r}$ and $\varepsilon_{0}$ are the relative permittivity of the perovskite and vacuum permittivity respectively. $V$ is the applied forward bias and $k_{B} T$ is the average thermal energy per particle. The characteristic $1 / 2 k_{B} T$ slope of an accumulation capacitance has been seen for the LF capacitance ${ }^{85,87}$ and is shown in Figure 1.13(a). This capacitance is therefore referred to as an interfacial or surface capacitance $C_{s}$, as seen in the EC of Figure 1.13. However, the origins of the large LF capacitance at SC is unclear, where carrier densities are minimized due to the fact that the Fermi levels at the contacts are aligned. 


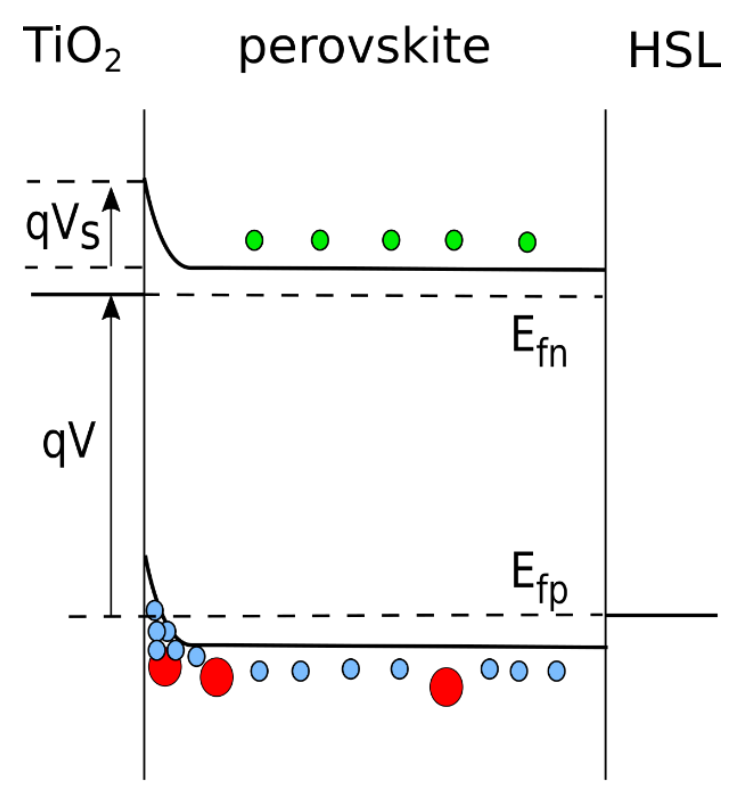

Figure 1.14 Band diagram of a PSC at large forward bias, with accumulation of majority holes (blue) and anions (red) at the $\mathrm{TiO}_{2} /$ perovskite interface, creating an electrostatic potential $V_{S}$ due to the upward band bending. Note that flat Fermi levels are drawn under the assumption of a very large diffusion coefficient for the electrons (green).

The capacitance observed at high frequencies is seen to be of the order of $10^{-7} \mathrm{~F} \cdot \mathrm{cm}^{2}$ and does not evolve with voltage or light intensity, as seen in Figure 1.13(a). This capacitance has been seen to evolve inversely with absorber thickness ${ }^{31}$ (see Figure 1.13(a)), confirming that it is indeed the bulk dielectric capacitance, which corresponds to the polarization of the entire device akin to a parallel plate capacitor of the form of Equation 13. This bulk dielectric or geometric capacitance is referred to as $C_{g}$ in the EC of Figure 1.13. The HF and LF resistances at OC (Figure 1.13(b)), $R_{2}$ and $R_{1}$ respectively in the EC of Figure 1.13, are generally seen to evolve exponentially with voltage, typical of recombination resistances given by

$$
R_{\text {rec }}=\frac{k_{B} T}{q^{2} L B_{r e c} n_{0} e^{\left(q V / m k_{B} T\right)}}
$$

In Equation 15, $m$ is the ideality factor of recombination, with $m=1$ corresponding to band-to-band recombination and $m=2$ corresponding to recombination via traps. ${ }^{88}$ While the HF resistance has been ascribed to bulk band-to-band recombination ${ }^{89}$ with $m$ values close to 1 , the LF resistance shows inconsistent $m$ values and is strongly connected to the LF capacitance. Furthermore, as seen from the EC of Figure 1.13, these resistances are coupled due to the fact that they are not parallel elements, whose mechanism has not yet been clarified. The series resistance $R_{S}$ observed generally is of the order of 10-20 $\Omega \cdot \mathrm{cm}^{2}$.

\subsection{Photocurrent kinetics}


IMPS is another useful small perturbation technique that measures the modulated extracted photocurrent in response to an input of modulated light intensity (see Section 2.2). This light modulated technique is a direct reflection of the EQE of the solar cell and is particularly useful in the case of PSCs, which are known to suffer from degradation in performance upon extended application of voltage such as in IS measurements. IMPS was used extensively to study electronic transport in DSSCs. The shielding of the electrical field within the mesoporous $\mathrm{TiO}_{2}$ layer by the ions in the electrolyte meant that transport was predominantly diffusive. ${ }^{90}$ This diffusive transport has a characteristic frequency given by

$$
\omega_{d}=\frac{D}{L^{2}}
$$

where $D$ is the diffusion coefficient of the electronic carrier and $L$ is the semiconductor layer thickness. In IMPS measurements, at frequencies greater than $\omega_{d}$, the electronic carrier is unable to reach the collecting contact and this shows a 45 degree straight line from the origin in an IMPS Q-plane plot, allowing decoupling of transport phenomena. ${ }^{91-}$ ${ }^{92}$ This response is also observed in IS measurements. ${ }^{93}$ At frequencies lower than $\omega_{d}$, an arc is formed in the upper quadrant of the Q-plane plot whose time constant is a product of the series resistance $R_{S}$ and the geometric capacitance $C_{g}$. In addition to transport processes, the IMPS spectra can provide information regarding the photovoltage distribution within the solar cell due to the modulated photon flux that can be modelled in terms of a current source.

However, in the case of PSCs, a true understanding of the IMPS response is lacking. Figure 1.15 shows the typical spectra observed in IMPS measurements of PSCs at SC. Figure 1.15(a) and 1.15(b) are the most common spectra, with the occurrence of two arcs in the upper quadrant in the Q-plane plot, or arcs in the upper and lower quadrant. In some cases, three arcs have been observed in the upper quadrant.

(a)

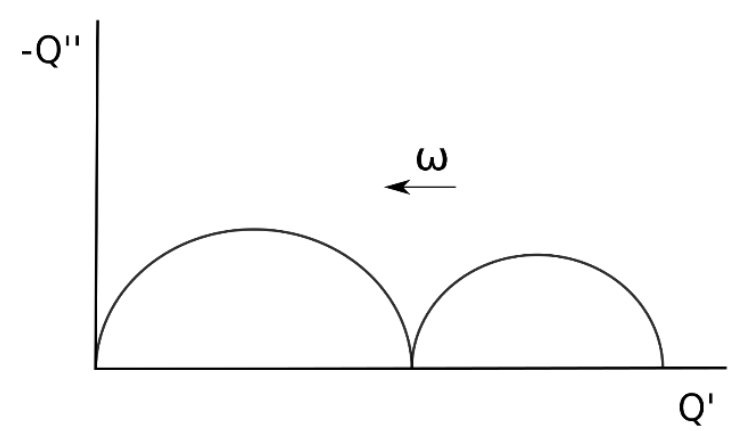

(b)

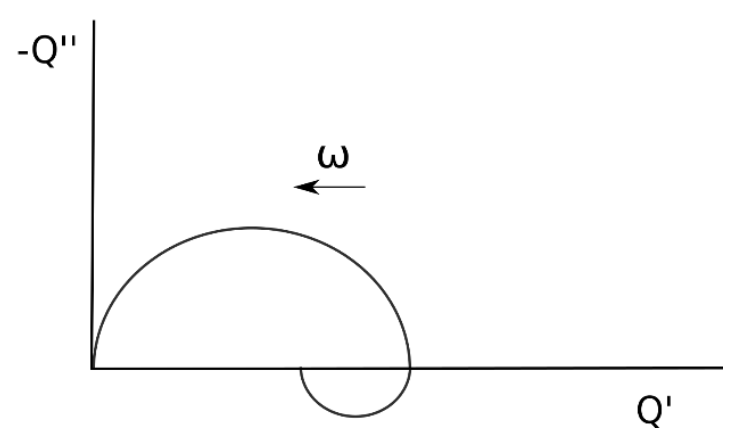

Figure 1.15 Typical IMPS spectra observed at SC conditions. (a) Two arcs in the upper quadrant and (b) low frequency arc in the lower quadrant of an IMPS Q-plane plot.

Generally, the high frequency arc in the upper quadrant has been ascribed to transport of electronic carriers in the perovskite layer ${ }^{94-95}$ while the low frequency arcs in the upper 
quadrant have been ascribed to transport of electrons in the mesoporous $\mathrm{TiO}_{2}$ layer, ${ }^{94,} 96$ and ionic diffusion. ${ }^{48,97}$ The arc in the lower quadrant has been tentatively ascribed to slow interfacial recombination. ${ }^{98}$ However, the rapid electronic transport observed ${ }^{70}$ coupled with the thin film configuration of PSCs raise doubts regarding several of the above mentioned interpretations. For example, taking a diffusion coefficient of $1 \mathrm{~cm}^{2} \mathrm{~s}^{-1}$ from reported mobility values ${ }^{99}$ of the electrons in MAPI and a film thickness of $300 \mathrm{~nm}$, the frequency of diffusion can be estimated from Equation 16 as $\sim 1.1 \times 10^{9} \mathrm{~Hz}$. This frequency is much faster than the perturbation frequencies used in both IMPS and IS measurements. A similar argument can be made for the diffusion of electrons in the mesoporous $\mathrm{TiO}_{2}$ layer through the thickness of the MAPI layer. While electronic transport in the PSC is likely too fast to be observed from IMPS and small perturbation techniques in general, the observation of the slow diffusion of the ions (reported diffusion coefficient ${ }^{26}$ of $\sim 10^{-8} \mathrm{~cm}^{2} \mathrm{~s}^{-1}$ ) cannot be ruled out. In general, the decoupling of the ionic and electronic phenomena in PSCs from small perturbation techniques is not straightforward and care must be taken while providing interpretations of the response. In addition, the IMPS Q-plane plots (Figure 1.15(b)) show a singular reduction in the external quantum efficiency (EQE) at very low frequencies, which has significant ramifications for the general measurement procedures of the EQE for PSCs and also the underlying physics governing this process. Therefore, there exist several open questions regarding the origin and significance of the arcs observed in the IMPS spectra of PSCs. 


\subsubsection{Device Modelling}

(a)

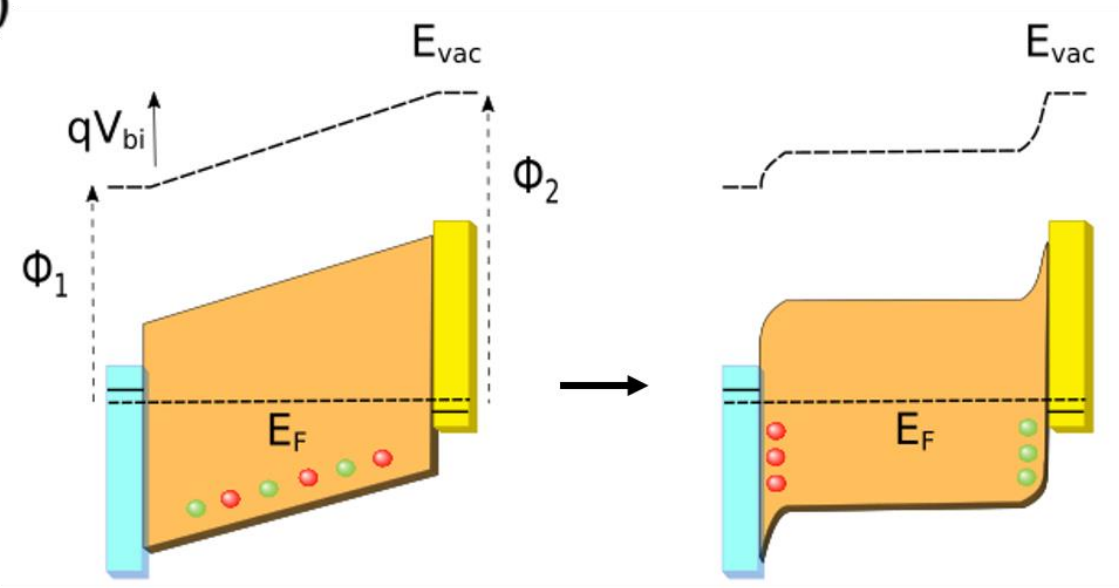

(b)

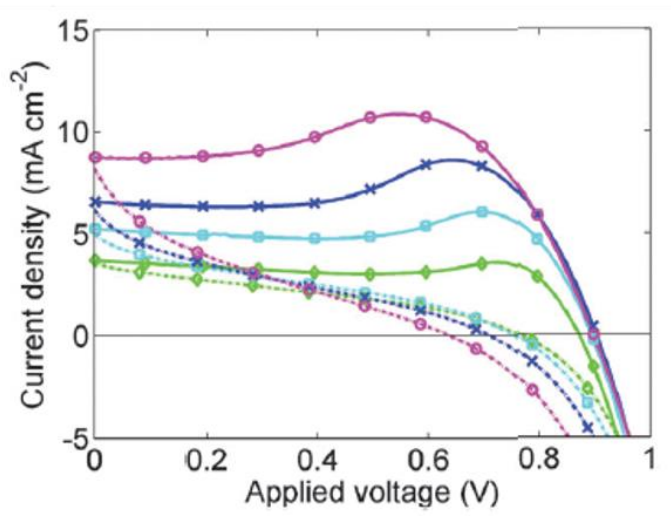

(c)

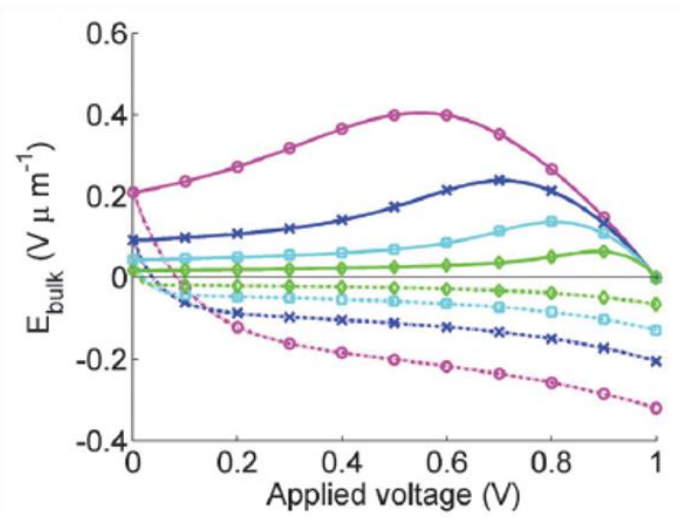

Figure 1.16 (a) n-i-p heterojunction model of a PSC with built-in electrical field through the absorber that is the difference between the workfunctions $\phi_{1}$ and $\phi_{2}$ of the external contacts, followed by ionic drift (cations - green, anions - red) along the field to yield an equilibrium field-free bulk. Adapted from Ref. 11 with permission, Copyright 2018, WILEY VCH Verlag GmbH \& Co. KGaA, Weinheim. (b) Simulated $j-V$ curves from forward bias and (c) corresponding electrical field in the bulk. Solid lines and dashed lines show FR and RF scans respectively. Magenta, blue, cyan and green colours corresponding to scan rates of 1000, 500, 250 and $100 \mathrm{mVs}^{-1}$ respectively. (b) and (c) adapted from Ref. 38, licensed by CC by 3.0, https://creativecommons.org/licenses/by/3.0/.

In order to simulate the unique features of the PSC $j-V$ response (summarized in Figure 1.7), a model of the solar cell must be created accounting for the transport, recombination and accumulation of the electronic carriers, while accounting for ionic drift and its effects on these parameters. This is carried out by solving the drift-diffusion equations coupled with the Poisson equation that yields the potential profile in the PSC.

One of the earliest works involved modelling the ESL/perovskite/HSL layer as a n-i$\mathrm{p}$ heterojunction. ${ }^{100}$ This assumes that the perovskite absorber is an intrinsic 
semiconductor with a built-in electrical field through the absorber layer that is the difference between the workfunctions of the ESL and the HSL (Figure 1.16(a)). Furthermore, hole and electron currents are assumed to be blocked at the ESL and HSL respectively and the ionic current is zero into the contacts. The Poisson equation was solved initially only for the ions at a stabilized potential $\left(V_{\text {stab }}\right)$, whose distribution was then fixed, following which the drift-diffusion equations and the Poisson equations were solved for the electronic carriers. This method is akin to establishing a steady state, where the ions redistribute and then performing a fast $j-V$ scan, which does not allow the ions time to redistribute and hence can be considered fixed. The ions are seen to shield the built-in electrical field by accumulating at the contacts forming sharp potential drops, leading to a bulk field-free region and the external voltage directly controlling the tilting of this field-free region in the absorber, which can be favourable for collecting charges or not, which obviously leads to a strong dependence of the $j-V$ curve on $V_{\text {stab }}$. An advanced model ${ }^{38}$ that works on the same principles of the $n-i-p$ model but solves the coupled drift-diffusion and Poisson equations for electronic carriers and ions yields the observed $j-V$ curve dependence on scan rate (Figure 1.16(b) and 1.16(c)), where the bulk electrical field is compensated throughout the scan at slow scan rates, intermediate scan rates yielding larger hysteresis and fast scan rates yielding low hysteresis due to the ions not having enough time to redistribute, as mentioned earlier. Similar models involve the simplifying assumption ${ }^{51,101}$ of flat sheets of charge, termed a dipolar distribution, at the contacts that create the same shielding effect, though it must be noted that such a distribution cannot yield a flat Fermi level (zero current) of the ions at steady state.

While these models reproduce some of the features of the hysteresis observed in PSCs, they are unable to explain the distinct recombination features including an extremely slow decay of the $V_{o c}$ (Figure 1.11(b)), an apparently limitless evolution of the LF capacitance with both light intensity and voltage and the strong role of the contacts (inverted configuration shows minimal hysteresis) in the observed performance. Furthermore, the $\mathrm{n}$ - $\mathrm{i}-\mathrm{p}$ model does not account for the effects of the strong injection/extraction barriers at the interfaces due to the ions on the extracted current.

Another major factor that has been attributed to the hysteresis in PSCs is trapping and de-trapping effects. ${ }^{100,102-103}$ While the effect of traps cannot be ruled out completely, it must be noted that the LF capacitance observed from IS measurements (Figure 1.13(a), $1.13(\mathrm{c})$ ) is extremely large, in the order of $\mathrm{mF}$, which yields abnormally large trap densities. Furthermore, this capacitance evolves exponentially with voltage, while a density of traps shows a characteristic maxima in capacitance at half occupancy. In addition, the timescale of hysteresis observed in the order of seconds indicates de-trapping rates of the same order, which is unrealistically slow. All these points cast strong doubts over the role of traps in the $j-V$ response and hysteretic phenomena observed in PSCs.

In summary, there is a strong need for a model that captures the hysteretic trends of the PSC based on specific experimental parameters while simultaneously accounting for the unique slow recombination and capacitance features that are characteristic of the PSCs. 


\subsubsection{References}

1. International Energy Outlook 2017. United States Energy Information Administration: 2017.

2. Shafiee, S.; Topal, E. When will fossil fuel reserves be diminished? Energy Policy 2009, 37 (1), 181-189.

3. Key world energy statistics 2017. International Energy Agency 2017.

4. Henbest, S.; Giannakopoulou, E.; Cuming, V. New Energy Outlook 2015. Bloomberg Finance LP 2015.

5. Philipps, S.; Ise, F.; Warmuth, W. Fraunhofer ISE: Photovoltaics Report 2018.

6. Annual Energy Outlook 2017. United States Energy Information Administration: 2017.

7. Kojima, A.; Teshima, K.; Shirai, Y.; Miyasaka, T. Organometal Halide Perovskites as Visible-Light Sensitizers for Photovoltaic Cells. Journal of the American Chemical Society 2009, 131 (17), 6050-6051.

8. Kim, H.-S.; Lee, C.-R.; Im, J.-H.; Lee, K.-B.; Moehl, T.; Marchioro, A.; Moon, S.-J.; Humphry-Baker, R.; Yum, J.-H.; Moser, J. E.; Grätzel, M.; Park, N.-G. Lead Iodide Perovskite Sensitized All-Solid-State Submicron Thin Film Mesoscopic Solar Cell with Efficiency Exceeding 9\%. Scientific Reports 2012, 2, 591.

9. Green, M. A.; Hishikawa, Y.; Dunlop, E. D.; Levi, D. H.; Hohl-Ebinger, J.; HoBaillie, A. W. Y. Solar cell efficiency tables (version 52). Progress in Photovoltaics: Research and Applications 2018, 26 (7), 427-436.

10. Rong, Y.; Ming, Y.; Ji, W.; Li, D.; Mei, A.; Hu, Y.; Han, H. Toward industrialscale production of perovskite solar cells: screen printing, slot-die coating, and emerging techniques. The Journal of Physical Chemistry Letters 2018, 9 (10), 2707-2713.

11. Lopez-Varo, P.; Jiménez-Tejada, J. A.; García-Rosell, M.; Ravishankar, S.; Garcia-Belmonte, G.; Bisquert, J.; Almora, O. Device physics of hybrid perovskite solar cells: theory and experiment. Advanced Energy Materials 2018, 8 (14), 1702772.

12. Almora, O.; Vaillant-Roca, L.; Garcia-Belmonte, G. Perovskite Solar Cells: A brief Introduction and some Remarks. Revista Cubana de Física; Vol 34 No 1 (2017) 2017.

13. Malinkiewicz, O.; Yella, A.; Lee, Y. H.; Espallargas, G. M.; Graetzel, M.; Nazeeruddin, M. K.; Bolink, H. J. Perovskite solar cells employing organic chargetransport layers. Nature Photonics 2014, 8 (2), 128.

14. Kim, H.-S.; Lee, C.-R.; Im, J.-H.; Lee, K.-B.; Moehl, T.; Marchioro, A.; Moon, S.-J.; Humphry-Baker, R.; Yum, J.-H.; Moser, J. E. Lead iodide perovskite sensitized allsolid-state submicron thin film mesoscopic solar cell with efficiency exceeding $9 \%$. Scientific Reports 2012, 2, 591. 
15. Eperon, G. E.; Stranks, S. D.; Menelaou, C.; Johnston, M. B.; Herz, L. M.; Snaith, H. J. Formamidinium lead trihalide: a broadly tunable perovskite for efficient planar heterojunction solar cells. Energy \& Environmental Science 2014, 7 (3), 982-988.

16. Tvingstedt, K.; Malinkiewicz, O.; Baumann, A.; Deibel, C.; Snaith, H. J.; Dyakonov, V.; Bolink, H. J. Radiative efficiency of lead iodide based perovskite solar cells. Scientific Reports 2014, 4, 6071.

17. Shockley, W.; Queisser, H. J. Detailed balance limit of efficiency of p-n junction solar cells. Journal of Applied Physics 1961, 32 (3), 510-519.

18. Tress, W.; Marinova, N.; Inganäs, O.; Nazeeruddin, M. K.; Zakeeruddin, S. M.; Graetzel, M. Predicting the open-circuit voltage of $\mathrm{CH}_{3} \mathrm{NH}_{3} \mathrm{PbI}_{3}$ perovskite solar cells using electroluminescence and photovoltaic quantum efficiency spectra: the role of radiative and non-radiative recombination. Advanced Energy Materials 2015, 5 (3), 1400812.

19. Park, N.-G. Perovskite solar cells: an emerging photovoltaic technology. Materials Today 2015, 18 (2), 65-72.

20. Jung, H. S.; Park, N.-G. Perovskite Solar Cells: From Materials to Devices. Small 2015, 11 (1), 10-25.

21. Noh, J. H.; Im, S. H.; Heo, J. H.; Mandal, T. N.; Seok, S. I. Chemical Management for Colorful, Efficient, and Stable Inorganic-Organic Hybrid Nanostructured Solar Cells. Nano Letters 2013, 13 (4), 1764-1769.

22. Yuan, Y.; Huang, J. Ion migration in organometal trihalide perovskite and its impact on photovoltaic efficiency and stability. Accounts of Chemical Research 2016, 49 (2), 286-293.

23. Eames, C.; Frost, J. M.; Barnes, P. R.; O'regan, B. C.; Walsh, A.; Islam, M. S. Ionic transport in hybrid lead iodide perovskite solar cells. Nature Communications 2015, 6, 7497.

24. Senocrate, A.; Moudrakovski, I.; Kim, G. Y.; Yang, T. Y.; Gregori, G.; Grätzel, M.; Maier, J. The nature of ion conduction in methylammonium lead iodide: a multimethod approach. Angewandte Chemie 2017, 129 (27), 7863-7867.

25. Li, C.; Guerrero, A.; Zhong, Y.; Gräser, A.; Luna, C. A. M.; Köhler, J.; Bisquert, J.; Hildner, R.; Huettner, S. Real-Time Observation of Iodide Ion Migration in Methylammonium Lead Halide Perovskites. Small 2017, 13 (42), 1701711.

26. Peng, W.; Aranda, C.; Bakr, O. M.; Garcia-Belmonte, G.; Bisquert, J.; Guerrero, A. Quantification of Ionic Diffusion in Lead Halide Perovskite Single Crystals. ACS Energy Letters 2018.

27. Li, C.; Guerrero, A.; Huettner, S.; Bisquert, J. Unravelling the role of vacancies in lead halide perovskite through electrical switching of photoluminescence. Nature 
communications 2018, 9 (1), 5113.

28. Sanchez, R. S.; Gonzalez-Pedro, V.; Lee, J.-W.; Park, N.-G.; Kang, Y. S.; MoraSero, I.; Bisquert, J. Slow dynamic processes in lead halide perovskite solar cells. Characteristic times and hysteresis. The Journal of Physical Chemistry Letters 2014, 5 (13), 2357-2363.

29. Chen, B.; Yang, M.; Zheng, X.; Wu, C.; Li, W.; Yan, Y.; Bisquert, J.; GarciaBelmonte, G.; Zhu, K.; Priya, S. Impact of capacitive effect and ion migration on the hysteretic behavior of perovskite solar cells. The Journal of Physical Chemistry Letters 2015, 6 (23), 4693-4700.

30. Chen, B.; Yang, M.; Priya, S.; Zhu, K. Origin of J-V Hysteresis in Perovskite Solar Cells. The Journal of Physical Chemistry Letters 2016, 7 (5), 905-917.

31. Almora, O.; Zarazua, I.; Mas-Marza, E.; Mora-Sero, I.; Bisquert, J.; GarciaBelmonte, G. Capacitive Dark Currents, Hysteresis, and Electrode Polarization in Lead Halide Perovskite Solar Cells. The Journal of Physical Chemistry Letters 2015, 6 (9), 1645-1652.

32. Unger, E. L.; Hoke, E. T.; Bailie, C. D.; Nguyen, W. H.; Bowring, A. R.; Heumuller, T.; Christoforo, M. G.; McGehee, M. D. Hysteresis and transient behavior in current-voltage measurements of hybrid-perovskite absorber solar cells. Energy \& Environmental Science 2014, 7 (11), 3690-3698.

33. Ono, L. K.; Raga, S. R.; Wang, S.; Kato, Y.; Qi, Y. Temperature-dependent hysteresis effects in perovskite-based solar cells. Journal of Materials Chemistry A 2015, 3 (17), 9074-9080.

34. Nemnes, G. A.; Besleaga, C.; Tomulescu, A. G.; Pintilie, I.; Pintilie, L.; Torfason, K.; Manolescu, A. Dynamic electrical behavior of halide perovskite based solar cells. Solar Energy Materials and Solar Cells 2017, 159, 197-203.

35. Tress, W.; Marinova, N.; Moehl, T.; Zakeeruddin, S. M.; Nazeeruddin, M. K.; Gratzel, M. Understanding the rate-dependent J-V hysteresis, slow time component, and aging in $\mathrm{CH}_{3} \mathrm{NH}_{3} \mathrm{PbI}_{3}$ perovskite solar cells: the role of a compensated electric field. Energy \& Environmental Science 2015, 8 (3), 995-1004.

36. Correa Baena, J. P.; Steier, L.; Tress, W.; Saliba, M.; Neutzner, S.; Matsui, T.; Giordano, F.; Jacobsson, T. J.; Srimath Kandada, A. R.; Zakeeruddin, S. M.; Petrozza, A.; Abate, A.; Nazeeruddin, M. K.; Gratzel, M.; Hagfeldt, A. Highly efficient planar perovskite solar cells through band alignment engineering. Energy \& Environmental Science 2015, 8 (10), 2928-2934.

37. Christians, J. A.; Manser, J. S.; Kamat, P. V. Best Practices in Perovskite Solar Cell Efficiency Measurements. Avoiding the Error of Making Bad Cells Look Good. The Journal of Physical Chemistry Letters 2015, 6 (5), 852-857. 
38. Richardson, G.; O'Kane, S. E.; Niemann, R. G.; Peltola, T. A.; Foster, J. M.; Cameron, P. J.; Walker, A. B. Can slow-moving ions explain hysteresis in the currentvoltage curves of perovskite solar cells? Energy \& Environmental Science 2016, 9 (4), $1476-1485$.

39. Tress, W.; Correa Baena, J. P.; Saliba, M.; Abate, A.; Graetzel, M. Inverted Current-Voltage Hysteresis in Mixed Perovskite Solar Cells: Polarization, Energy Barriers, and Defect Recombination. Advanced Energy Materials 2016, 6 (19), 1600396.

40. Nemnes, G. A.; Besleaga, C.; Stancu, V.; Dogaru, D. E.; Leonat, L. N.; Pintilie, L.; Torfason, K.; Ilkov, M.; Manolescu, A.; Pintilie, I. Normal and inverted hysteresis in perovskite solar cells. The Journal of Physical Chemistry C 2017.

41. Shen, H.; Jacobs, D. A.; Wu, Y.; Duong, T.; Peng, J.; Wen, X.; Fu, X.; Karuturi, S. K.; White, T. P.; Weber, K. Inverted hysteresis in $\mathrm{CH}_{3} \mathrm{NH}_{3} \mathrm{PbI}_{3}$ solar cells: role of stoichiometry and band alignment. The Journal of Physical Chemistry Letters 2017, 8 (12), 2672-2680.

42. Zhao, Y.; Liang, C.; Zhang, H.; Li, D.; Tian, D.; Li, G.; Jing, X.; Zhang, W.; Xiao, W.; Liu, Q.; Zhang, F.; He, Z. Anomalously large interface charge in polarity-switchable photovoltaic devices: an indication of mobile ions in organic-inorganic halide perovskites. Energy \& Environmental Science 2015, 8 (4), 1256-1260.

43. Chen, H.-W.; Sakai, N.; Ikegami, M.; Miyasaka, T. Emergence of Hysteresis and Transient Ferroelectric Response in Organo-Lead Halide Perovskite Solar Cells. The Journal of Physical Chemistry Letters 2015, 6 (1), 164-169.

44. Kim, H.-S.; Park, N.-G. Parameters affecting I-V hysteresis of $\mathrm{CH}_{3} \mathrm{NH}_{3} \mathrm{PbI}_{3}$ perovskite solar cells: effects of perovskite crystal size and mesoporous $\mathrm{TiO}_{2}$ layer. The Journal of Physical Chemistry Letters 2014, 5 (17), 2927-2934.

45. Zhao, C.; Chen, B.; Qiao, X.; Luan, L.; Lu, K.; Hu, B. Revealing underlying processes involved in light soaking effects and hysteresis phenomena in perovskite solar cells. Advanced Energy Materials 2015, 5 (14), 1500279.

46. Barrows, A. T.; Pearson, A. J.; Kwak, C. K.; Dunbar, A. D.; Buckley, A. R.; Lidzey, D. G. Efficient planar heterojunction mixed-halide perovskite solar cells deposited via spray-deposition. Energy \& Environmental Science 2014, 7 (9), 2944-2950.

47. Samu, G. F.; Janáky, C.; Kamat, P. V. A Victim of Halide Ion Segregation. How Light Soaking Affects Solar Cell Performance of Mixed Halide Lead Perovskites. ACS Energy Letters 2017, 2 (8), 1860-1861.

48. Contreras, L.; Idigoras, J.; Todinova, A.; Salado, M.; Kazim, S.; Ahmad, S.; Anta, J. A. Specific cation interactions as the cause of slow dynamics and hysteresis in dye and perovskite solar cells: a small-perturbation study. Physical Chemistry Chemical Physics 2016, 18 (45), 31033-31042. 
49. Meloni, S.; Moehl, T.; Tress, W.; Franckevičius, M.; Saliba, M.; Lee, Y. H.; Gao, P.; Nazeeruddin, M. K.; Zakeeruddin, S. M.; Rothlisberger, U. Ionic polarization-induced current-voltage hysteresis in $\mathrm{CH}_{3} \mathrm{NH}_{3} \mathrm{PbX}_{3}$ perovskite solar cells. Nature communications 2016, 7, 10334.

50. Bryant, D.; Wheeler, S.; O’Regan, B. C.; Watson, T.; Barnes, P. R.; Worsley, D.; Durrant, J. Observable hysteresis at low temperature in "hysteresis free" organicinorganic lead halide perovskite solar cells. The Journal of Physical Chemistry Letters 2015, 6 (16), 3190-3194.

51. Belisle, R. A.; Nguyen, W. H.; Bowring, A. R.; Calado, P.; Li, X.; Irvine, S. J. C.; McGehee, M. D.; Barnes, P. R. F.; O'Regan, B. C. Interpretation of inverted photocurrent transients in organic lead halide perovskite solar cells: proof of the field screening by mobile ions and determination of the space charge layer widths. Energy \& Environmental Science 2017, 10 (1), 192-204.

52. Hermes, I. M.; Hou, Y.; Bergmann, V. W.; Brabec, C. J.; Weber, S. A. The Interplay of Contact Layers: How the Electron Transport Layer Influences Interfacial Recombination and Hole Extraction in Perovskite Solar Cells. The Journal of Physical Chemistry Letters 2018, 9 (21), 6249-6256.

53. Sanchez, R. S.; Mas-Marza, E. Light-induced effects on Spiro-OMeTAD films and hybrid lead halide perovskite solar cells. Solar Energy Materials and Solar Cells 2016, 158, 189-194.

54. Shao, Y.; Xiao, Z.; Bi, C.; Yuan, Y.; Huang, J. Origin and elimination of photocurrent hysteresis by fullerene passivation in $\mathrm{CH}_{3} \mathrm{NH}_{3} \mathrm{PbI}_{3}$ planar heterojunction solar cells. Nature Communications 2014, 5, 5784.

55. Chiang, C.-H.; Wu, C.-G. Bulk heterojunction perovskite-PCBM solar cells with high fill factor. Nature Photonics 2016, 10 (3), 196-200.

56. Zarazua, I.; Han, G.; Boix, P. P.; Mhaisalkar, S.; Fabregat-Santiago, F.; MoraSeró, I.; Bisquert, J.; Garcia-Belmonte, G. Surface Recombination and Collection Efficiency in Perovskite Solar Cells from Impedance Analysis. The Journal of Physical Chemistry Letters 2016, 7 (24), 5105-5113.

57. Kim, H.-S.; Jang, I.-H.; Ahn, N.; Choi, M.; Guerrero, A.; Bisquert, J.; Park, N.-G. Control of $\mathrm{I}-\mathrm{V}$ hysteresis in $\mathrm{CH}_{3} \mathrm{NH}_{3} \mathrm{PbI}_{3}$ perovskite solar cell. The Journal of Physical Chemistry Letters 2015, 6 (22), 4633-4639.

58. Guerrero, A.; Bou, A.; Matt, G.; Almora, O.; Heumüller, T.; Garcia-Belmonte, G.; Bisquert, J.; Hou, Y.; Brabec, C. Switching Off Hysteresis in Perovskite Solar Cells by Fine-Tuning Energy Levels of Extraction Layers. Advanced Energy Materials 2018, 8 (21), 1703376.

59. Würfel, U.; Cuevas, A.; Würfel, P. Charge carrier separation in solar cells. IEEE 
Journal of Photovoltaics 2015, 5 (1), 461-469.

60. Juarez-Perez, E. J.; Wußler, M.; Fabregat-Santiago, F.; Lakus-Wollny, K.; Mankel, E.; Mayer, T.; Jaegermann, W.; Mora-Sero, I. Role of the Selective Contacts in the Performance of Lead Halide Perovskite Solar Cells. The Journal of Physical Chemistry Letters 2014, 5 (4), 680-685.

61. Rau, U. Reciprocity relation between photovoltaic quantum efficiency and electroluminescent emission of solar cells. Physical Review B 2007, 76 (8), 085303.

62. Bisquert, J. The Physics of Solar Cells: Perovskites, Organics, and Photovoltaic Fundamentals. CRC Press: 2017.

63. Shockley, W.; Read Jr, W. Statistics of the recombinations of holes and electrons. Physical Review 1952, 87 (5), 835.

64. Stranks, S. D.; Burlakov, V. M.; Leijtens, T.; Ball, J. M.; Goriely, A.; Snaith, H. J. Recombination kinetics in organic-inorganic perovskites: excitons, free charge, and subgap states. Physical Review Applied 2014, 2 (3), 034007.

65. Zaban, A.; Greenshtein, M.; Bisquert, J. Determination of the electron lifetime in nanocrystalline dye solar cells by open-circuit voltage decay measurements. ChemPhysChem 2003, 4 (8), 859-864.

66. Baumann, A.; Tvingstedt, K.; Heiber, M.; Väth, S.; Momblona, C.; Bolink, H.; Dyakonov, V. Persistent photovoltage in methylammonium lead iodide perovskite solar cells. APL Materials 2014, 2 (8), 081501.

67. Gottesman, R.; Lopez-Varo, P.; Gouda, L.; Jimenez-Tejada, J. A.; Hu, J.; Tirosh, S.; Zaban, A.; Bisquert, J. Dynamic Phenomena at Perovskite/Electron-Selective Contact Interface as Interpreted from Photovoltage Decays. Chem 2016, 1 (5), 776-789.

68. Miyata, A.; Mitioglu, A.; Plochocka, P.; Portugall, O.; Wang, J. T.-W.; Stranks, S. D.; Snaith, H. J.; Nicholas, R. J. Direct measurement of the exciton binding energy and effective masses for charge carriers in organic-inorganic tri-halide perovskites. Nature Physics 2015, 11 (7), 582-587.

69. Lin, Q.; Armin, A.; Nagiri, R. C. R.; Burn, P. L.; Meredith, P. Electro-optics of perovskite solar cells. Nature Photonics 2015, 9 (2), 106.

70. Stranks, S. D.; Eperon, G. E.; Grancini, G.; Menelaou, C.; Alcocer, M. J.; Leijtens, T.; Herz, L. M.; Petrozza, A.; Snaith, H. J. Electron-hole diffusion lengths exceeding 1 micrometer in an organometal trihalide perovskite absorber. Science 2013, 342 (6156), 341-344.

71. Shi, D.; Adinolfi, V.; Comin, R.; Yuan, M.; Alarousu, E.; Buin, A.; Chen, Y.; Hoogland, S.; Rothenberger, A.; Katsiev, K.; Losovyj, Y.; Zhang, X.; Dowben, P. A.; Mohammed, O. F.; Sargent, E. H.; Bakr, O. M. Low trap-state density and long carrier diffusion in organolead trihalide perovskite single crystals. Science 2015, 347 (6221), 519. 
72. Sheng, R.; Ho-Baillie, A.; Huang, S.; Chen, S.; Wen, X.; Hao, X.; Green, M. A. Methylammonium Lead Bromide Perovskite-Based Solar Cells by Vapor-Assisted Deposition. The Journal of Physical Chemistry C 2015, 119 (7), 3545-3549.

73. Milot, R. L.; Eperon, G. E.; Snaith, H. J.; Johnston, M. B.; Herz, L. M. Temperature-dependent charge-carrier dynamics in $\mathrm{CH}_{3} \mathrm{NH}_{3} \mathrm{PbI}_{3}$ perovskite thin films. Advanced Functional Materials 2015, 25 (39), 6218-6227.

74. Hutter, E. M.; Eperon, G. E.; Stranks, S. D.; Savenije, T. J. Charge carriers in planar and meso-structured organic-inorganic perovskites: mobilities, lifetimes, and concentrations of trap states. The Journal of Physical Chemistry Letters 2015, 6 (15), 3082-3090.

75. Semonin, O. E.; Elbaz, G. A.; Straus, D. B.; Hull, T. D.; Paley, D. W.; van der Zande, A. M.; Hone, J. C.; Kymissis, I.; Kagan, C. R.; Roy, X. Limits of Carrier Diffusion in n-Type and p-Type $\mathrm{CH}_{3} \mathrm{NH}_{3} \mathrm{PbI}_{3}$ Perovskite Single Crystals. The Journal of Physical Chemistry Letters 2016, 7 (17), 3510-3518.

76. Dong, Q.; Fang, Y.; Shao, Y.; Mulligan, P.; Qiu, J.; Cao, L.; Huang, J. Electronhole diffusion lengths $>175 \mu \mathrm{m}$ in solution grown $\mathrm{CH}_{3} \mathrm{NH}_{3} \mathrm{PbI}_{3}$ single crystals. Science 2015, aaa5760.

77. Pitarch-Tena, D.; Ngo, T. T.; Vallés-Pelarda, M.; Pauporté, T.; Mora-Seró, I. Impedance Spectroscopy Measurements in Perovskite Solar Cells: Device Stability and Noise Reduction. ACS Energy Letters 2018, 3 (4), 1044-1048.

78. Fabregat-Santiago, F.; Kulbak, M.; Zohar, A.; Vallés-Pelarda, M.; Hodes, G.; Cahen, D.; Mora-Seró, I. Deleterious Effect of Negative Capacitance on the Performance of Halide Perovskite Solar Cells. ACS Energy Letters 2017, 2 (9), 2007-2013.

79. Zohar, A.; Kedem, N.; Levine, I.; Zohar, D.; Vilan, A.; Ehre, D.; Hodes, G.; Cahen, D. Impedance spectroscopic indication for solid state electrochemical reaction in $\left(\mathrm{CH}_{3} \mathrm{NH}_{3}\right) \mathrm{PbI}_{3}$ films. The Journal of Physical Chemistry Letters 2015, 7 (1), 191-197.

80. Guerrero, A.; Garcia-Belmonte, G.; Mora-Sero, I.; Bisquert, J.; Kang, Y. S.; Jacobsson, T. J.; Correa-Baena, J.-P.; Hagfeldt, A. Properties of Contact and Bulk Impedances in Hybrid Lead Halide Perovskite Solar Cells Including Inductive Loop Elements. The Journal of Physical Chemistry C 2016, 120 (15), 8023-8032.

81. Bisquert, J. A variable series resistance mechanism to explain the negative capacitance observed in impedance spectroscopy measurements of nanostructured solar cells. Physical Chemistry Chemical Physics 2011, 13 (10), 4679-4685.

82. Anaya, M.; Zhang, W.; Hames, B. C.; Li, Y.; Fabregat-Santiago, F.; Calvo, M. E.; Snaith, H. J.; Míguez, H.; Mora-Seró, I. Electron injection and scaffold effects in perovskite solar cells. Journal of Materials Chemistry C 2017, 5 (3), 634-644.

83. Bard, A. J.; Faulkner, L. R.; Leddy, J.; Zoski, C. G. Electrochemical methods: 
fundamentals and applications. wiley New York: 1980; Vol. 2.

84. Bergmann, V. W.; Guo, Y.; Tanaka, H.; Hermes, I. M.; Li, D.; Klasen, A.; Bretschneider, S. A.; Nakamura, E.; Berger, R. d.; Weber, S. A. Local time-dependent charging in a perovskite solar cell. ACS Applied Materials \& Interfaces 2016, 8 (30), 19402-19409.

85. Zarazua, I.; Bisquert, J.; Garcia-Belmonte, G. Light-induced space-charge accumulation zone as photovoltaic mechanism in perovskite solar cells. The Journal of Physical Chemistry Letters 2016, 7 (3), 525-528.

86. Mönch, W. Semiconductor surfaces and interfaces. Springer Science \& Business Media: 2013; Vol. 26.

87. Almora, O.; Cho, K. T.; Aghazada, S.; Zimmermann, I.; Matt, G. J.; Brabec, C. J.; Nazeeruddin, M. K.; Garcia-Belmonte, G. Discerning recombination mechanisms and ideality factors through impedance analysis of high-efficiency perovskite solar cells. Nano Energy 2018, 48, 63-72.

88. Sze, S. M.; Ng, K. K. Physics of semiconductor devices. John wiley \& sons: 2006.

89. Correa-Baena, J.-P.; Turren-Cruz, S.-H.; Tress, W.; Hagfeldt, A.; Aranda, C.; Shooshtari, L.; Bisquert, J.; Guerrero, A. Changes from Bulk to Surface Recombination Mechanisms between Pristine and Cycled Perovskite Solar Cells. ACS Energy Letters 2017, 2, 681-688.

90. Soedergren, S.; Hagfeldt, A.; Olsson, J.; Lindquist, S.-E. Theoretical Models for the Action Spectrum and the Current-Voltage Characteristics of Microporous Semiconductor Films in Photoelectrochemical Cells. The Journal of Physical Chemistry 1994, 98 (21), 5552-5556.

91. Ponomarev, E.; Peter, L. A generalized theory of intensity modulated photocurrent spectroscopy (IMPS). Journal of Electroanalytical Chemistry 1995, 396 (1-2), 219-226.

92. Dloczik, L.; Ileperuma, O.; Lauermann, I.; Peter, L. M.; Ponomarev, E. A.; Redmond, G.; Shaw, N. J.; Uhlendorf, I. Dynamic Response of Dye-Sensitized Nanocrystalline Solar Cells: Characterization by Intensity-Modulated Photocurrent Spectroscopy. The Journal of Physical Chemistry B 1997, 101 (49), 10281-10289.

93. Bisquert, J. Theory of the impedance of electron diffusion and recombination in a thin layer. The Journal of Physical Chemistry B 2002, 106 (2), 325-333.

94. Guillén, E.; Ramos, F. J.; Anta, J. A.; Ahmad, S. Elucidating TransportRecombination Mechanisms in Perovskite Solar Cells by Small-Perturbation Techniques. The Journal of Physical Chemistry C 2014, 118 (40), 22913-22922.

95. Giordano, F.; Abate, A.; Baena, J. P. C.; Saliba, M.; Matsui, T.; Im, S. H.; Zakeeruddin, S. M.; Nazeeruddin, M. K.; Hagfeldt, A.; Graetzel, M. Enhanced electronic properties in mesoporous $\mathrm{TiO}_{2}$ via lithium doping for high-efficiency perovskite solar 
cells. Nature Communications 2016, 7, 10379.

96. Todinova, A.; Idígoras, J.; Salado, M.; Kazim, S.; Anta, J. A. Universal Features of Electron Dynamics in Solar Cells with $\mathrm{TiO}_{2}$ Contact: From Dye Solar Cells to Perovskite Solar Cells. The Journal of Physical Chemistry Letters 2015, 6 (19), 39233930.

97. Correa-Baena, J.-P.; Anaya, M.; Lozano, G.; Tress, W.; Domanski, K.; Saliba, M.; Matsui, T.; Jacobsson, T. J.; Calvo, M. E.; Abate, A.; Grätzel, M.; Míguez, H.; Hagfeldt, A. Unbroken Perovskite: Interplay of Morphology, Electro-optical Properties, and Ionic Movement. Advanced Materials 2016, 28 (25), 5031-5037.

98. Pockett, A.; Eperon, G. E.; Peltola, T.; Snaith, H. J.; Walker, A.; Peter, L. M.; Cameron, P. J. Characterization of planar lead halide perovskite solar cells by impedance spectroscopy, open-circuit photovoltage decay, and intensity-modulated photovoltage/photocurrent spectroscopy. The Journal of Physical Chemistry C 2015, 119 (7), 3456-3465.

99. Herz, L. M. Charge-carrier mobilities in metal halide perovskites: fundamental mechanisms and limits. ACS Energy Letters 2017, 2 (7), 1539-1548.

100.van Reenen, S.; Kemerink, M.; Snaith, H. J. Modeling Anomalous Hysteresis in Perovskite Solar Cells. The Journal of Physical Chemistry Letters 2015, 6 (19), 38083814 .

101.Jacobs, D. A.; Wu, Y.; Shen, H.; Barugkin, C.; Beck, F. J.; White, T. P.; Weber, K.; Catchpole, K. R. Hysteresis phenomena in perovskite solar cells: the many and varied effects of ionic accumulation. Physical Chemistry Chemical Physics 2017, 19 (4), 30943103 .

102.Sherkar, T. S.; Momblona, C.; Gil-Escrig, L. n.; Ávila, J.; Sessolo, M.; Bolink, H. J.; Koster, L. J. A. Recombination in perovskite solar cells: significance of grain boundaries, interface traps, and defect ions. ACS energy letters 2017, 2 (5), 1214-1222.

103.Sherkar, T. S.; Momblona, C.; Gil-Escrig, L.; Bolink, H. J.; Koster, L. J. A. Improving Perovskite Solar Cells: Insights From a Validated Device Model. Advanced Energy Materials 2017, 7 (13), 1602432. 


\section{Chapter 2}

\section{Methods}

\subsection{Impedance Spectroscopy (IS)}

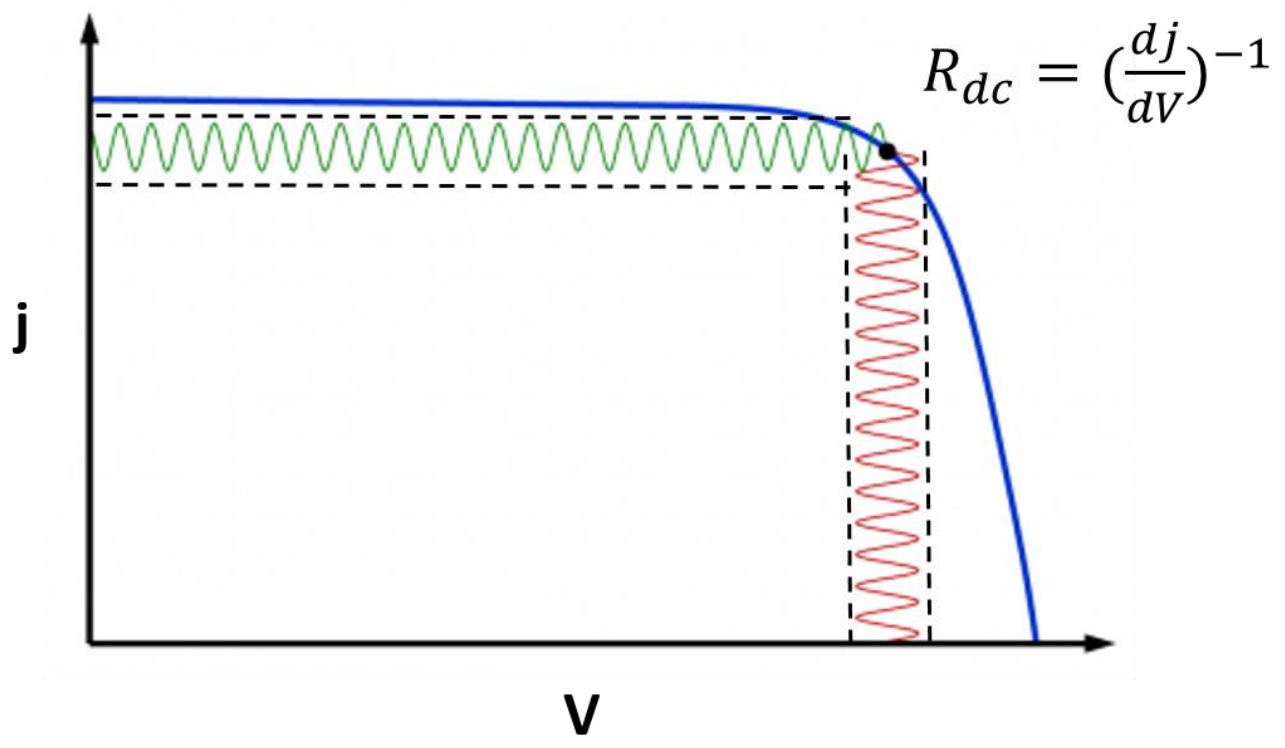

Figure 2.1 Illustration of the technique of impedance spectroscopy. A small perturbation of modulated voltage around a given steady state point (black dot) in the $j-V$ curve is applied and the resulting modulated current response is measured. The low frequency limit of the transfer function is the differential DC resistance at that point in the $j-V$ curve.

This technique involves the application of a small perturbation of modulated voltage $\tilde{V}=V_{0} e^{i \omega t}$, where $V_{0}$ is the amplitude of the wave, to a solar cell from a given steady state voltage $\bar{V}$ and measuring the corresponding modulated current density response $\tilde{J}$ superimposed on top of the steady state current $\bar{J}$. This response can be written using the Taylor series and small perturbation condition $(\bar{V} \gg \tilde{V})$ as

$$
\mathrm{j}(\bar{V}+\tilde{V})=\overline{\mathrm{j}}+\frac{d j(\bar{V})}{d \bar{V}} \tilde{V}+\frac{1}{2 !} \frac{d^{2} j(\bar{V})}{d \bar{V}^{2}} \tilde{V}^{2}+\frac{1}{3 !} \frac{d^{3} j(\bar{V})}{d \bar{V}^{3}} \tilde{V}^{3} \ldots \ldots
$$

which yields

$$
\tilde{J}=\frac{d j(\bar{V})}{d \bar{V}} \tilde{V}
$$

These conditions ensure that the current response is linear with the applied voltage perturbation, in the form of Equation 18. The current response can be shifted in phase with respect to the voltage perturbation as shown in Figure 2.1 and can be written as $j_{0} e^{i(\omega t+\phi)}$. Here, $\phi$ is the phase difference and $j_{0}$ is the amplitude of the modulated output. The modulated current density response is measured over a range of input 
frequencies $\omega$ of the modulated voltage, generally ranging between $1 \mathrm{MHz}-10 \mathrm{mHz}$. Therefore, the IS transfer function $Z\left(\Omega \cdot \mathrm{cm}^{2}\right)$ is formally defined as ${ }^{1}$

$Z(\omega)=\frac{\widetilde{V}(\omega)}{\tilde{\jmath}(\omega)}$

The IS transfer function is a complex number, whose LF limit is the DC differential resistance at the given steady state point of measurement, as shown in Figure 2.1. The great advantage of IS measurements is that it can be measured at several different conditions, such as over a range of voltages and in dark or illuminated conditions. This provides information of the solar cell at different points of operation, allowing to understand characteristic evolution of mechanistic timescales. The IS data is generally represented in the form of a Z-plane plot, ie: real part of $Z\left(Z^{\prime}\right)$ versus imaginary part of $Z\left(Z^{\prime \prime}\right)$ over the range of measured frequencies. This allows separating different internal mechanistic processes occurring on different characteristic timescales. These characteristic times can be represented in terms of combinations of passive electrical elements that yield time constants. These passive elements are electrical replicas of physical mechanisms occurring in the solar cell such as recombination and charge storage.
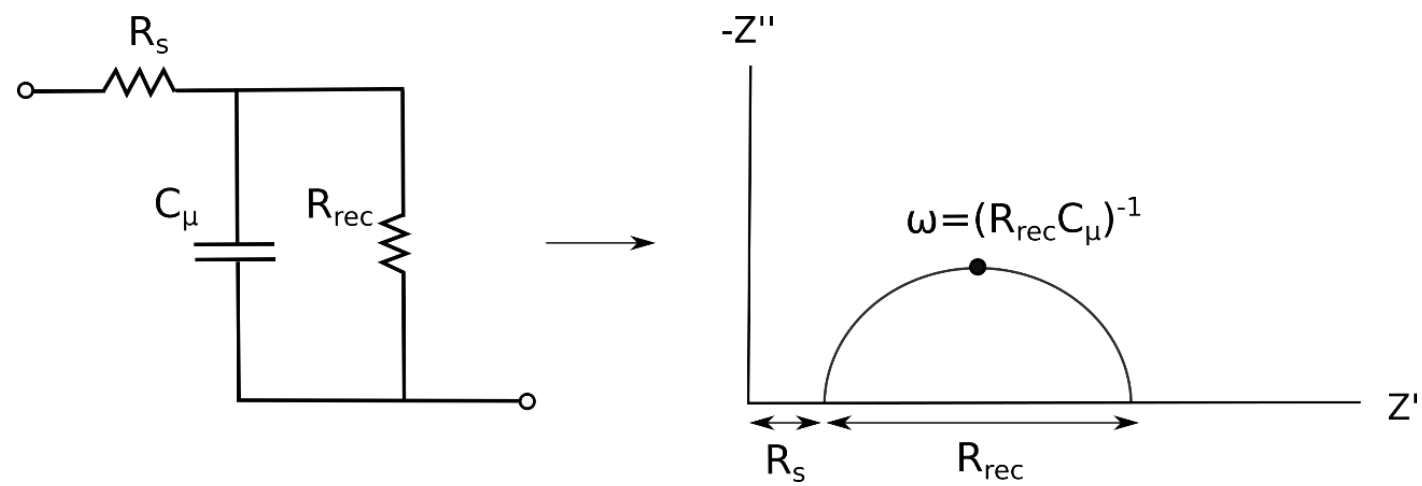

Figure 2.2 Basic solar cell electrical model consisting of series resistance $R_{S}$, chemical capacitance $C_{\mu}$ and recombination resistance $R_{\text {rec }}$ and corresponding Z-plane response.

As an example, Figure 2.2 shows the basic solar cell model that consists of a series resistance $R_{s}$, with a parallel combination of the chemical capacitance $C_{\mu}$ related to the filling of the density of states of the absorber and recombination resistance $R_{\text {rec }}$ that describes the density of recombination of electronic carriers. The Z-plane response consists of an arc in the upper quadrant whose width is given by $R_{\text {rec }}$ and which is displaced along the x-axis by a value equal to $R_{S}$. The ability to distinguish the value of the series resistance of the solar cell in a straightforward manner is in fact one of the major advantages of IS. Furthermore, the frequency of the maxima of the imaginary part, which is the top of the arc in the Z-plane plot in Figure 2.2, corresponds to the inverse of the time constant formed by the product of $R_{r e c}$ and $C_{\mu}$. Other processes occurring on different timescales can be added to the response of Figure 2.2 depending on their characteristic response, leading to the de-convolution of several complex phenomena using IS. In general, these characteristic times can provide information about fundamental processes and parameters in the solar cell. For example, the time constant shown in Figure 
2.2, the product of the recombination resistance and chemical capacitance, is in fact the fundamental lifetime $\tau_{\text {rec }}$ of recombination of minority carrier density $n$, given that the recombination $U$ is linear with the injected charge. This can be seen from the definition of the lifetime ${ }^{2}$

$$
\tau_{\text {rec }}=\left(\frac{d U}{d n}\right)^{-1}
$$

However, in more complex cases, such as when traps contribute to the net lifetime, the time constant in IS is more generally referred to as the response time, which is different from the lifetime. Another useful representation is the frequency dependent capacitance expressed as

$$
C(\omega)=\frac{1}{i \omega Z(\omega)}
$$

This representation allows the identification of the type of capacitances being observed over the range of frequencies. For example, for the simple circuit shown in Figure 2.2, at frequencies much faster than its characteristic frequency, the capacitor acts like a short circuit and the capacitance is essentially zero. Moving towards lower frequencies, the capacitance increases, reaching its maximum value and remaining constant. Therefore, the capacitance versus frequency plot generally shows a number of increments and plateaus depending on the timescales of different charge storage mechanisms. These include the space charge capacitance, the chemical capacitance, bulk dielectric capacitance and surface state capacitance, which can be identified from their singular evolution with voltage or sample thickness (see Figure 2.6(a)). This in turn allows extraction of useful parameters of the solar cell such as the doping density, density of states and dielectric constant.

IS was initially used in the field of photoelectrochemistry, in order to understand charge densities and transfer rates at semiconductor/electrolyte interfaces. It has since become a very useful tool to characterize solar cells, most famously in the field of the dye-sensitized solar cell (DSSC). Therefore, some seminal achievements of IS in this field are summarised in the subsequent sections to provide a glimpse of the power of this technique. 


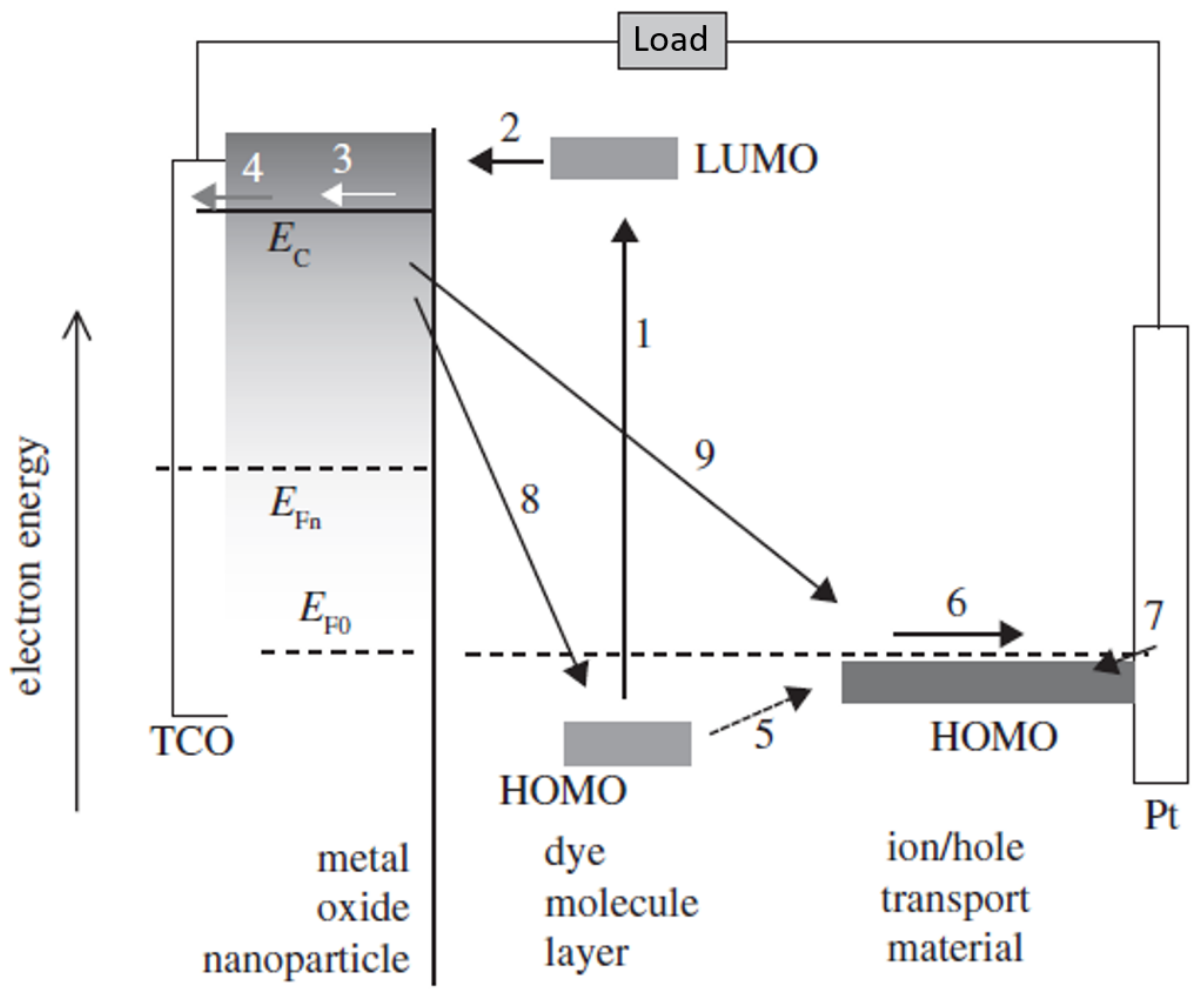

Figure 2.3 Schematic illustration of the different processes involved in the action of a dye-sensitized solar cell. (1) Absorption of incident light by dye molecules to generate electrons and holes in the highest occupied molecular orbital (HOMO) and lowest unoccupied molecular orbital (LUMO) respectively. (2) Transfer of photogenerated holes to the conduction band of the $\mathrm{TiO}_{2}$. (3) Transport of electrons within the $\mathrm{TiO}_{2}$. (4) Transfer of electrons from $\mathrm{TiO}_{2}$ to the TCO. (5) Transfer of photogenerated holes from the LUMO of the dye molecules to the redox couple in the electrolyte or the HOMO of a hole transport material. (6) Transport of holes within the electrolyte or hole transport material. (7) Recombination of electrons driven through the load with the holes. Recombination of electrons in the conduction band of $\mathrm{TiO}_{2}$ with (8) holes in the HOMO of the dye molecules and (9) holes in the electrolyte or HOMO of the hole transport material. Adapted with permission from Ref. 1.

The DSSC consists of a nanostructured, mesoporous $\mathrm{TiO}_{2}$ electrode deposited on top of a transparent conducting oxide (TCO) and is coated with photoactive dye molecules. This is further in contact with an electrolyte that consists of a $\mathrm{I}^{-} / \mathrm{I}_{3}{ }^{-}$redox couple. The circuit is completed by connecting the $\mathrm{TiO}_{2}$ electrode through a load to a platinum (Pt) electrode. Upon illumination, the photoactive dye molecules absorb the light and generate electron-hole pairs. These electrons are then transferred from the dye to the conduction band of the $\mathrm{TiO}_{2}$ electrode, travelling through the thickness of the electrode and collected by the TCO. The photogenerated holes are transferred to the electrolyte via the redox couple and transferred to the Pt electrode and reach the collected electrons to complete the circuit. The nanostructured, mesoporous nature of the $\mathrm{TiO}_{2}$ electrode is to maximise 
loading of the dye and hence absorption of incoming light by enhancing the surface area and to maximise contact of the electrolyte with the dye molecules to transfer the photogenerated holes, respectively. A schematic of the different processes occurring in a DSSC is shown in Figure 2.3.

IS measurements on DSSCs provided a large amount of information regarding the transport, recombination and capacitance characteristics of the electrons in the $\mathrm{TiO}_{2}$ electrode. This was possible due to knowledge of the fact that the electrons in the conduction band of the $\mathrm{TiO}_{2}$ are shielded from any internal electric fields due to the electrolyte ions surrounding it. ${ }^{3-4}$ Therefore, electrons reach the collecting contact by diffusion through the $\mathrm{TiO}_{2}$ layer. The efficiency of reaching the collecting contact is determined by the diffusion length, which accounts for the recombination of these electrons with holes in the electrolyte or in the dye molecules themselves. The combination of these two facets yields the diffusion-recombination model ${ }^{4}$, where the continuity equation is given by

$$
\frac{d n}{d t}=D \frac{d^{2} n}{d x^{2}}-k\left(n-n_{0}\right)+G
$$

where $n$ is the electron density and $n_{0}$ is the equilibrium electron density, $x$ is the position along the length $L$ of the semiconductor, $D$ is the diffusion coefficient of electrons, $k$ is the rate constant for recombination and $G$ is the generation rate of electron-hole pairs. The boundary conditions are given by the voltage-modulated electron density on one side

$$
n(\mathrm{x}=0)=n_{0} \exp \left(\frac{q V}{k_{B} T}\right)
$$

and a blocking or reflective boundary at the $\mathrm{TiO}_{2} /$ electrolyte interface

$$
\frac{d n}{d x}(x=L)=0
$$

The total extracted current is given by

$$
j=q D \frac{d n}{d x}(x=0)
$$

From Equations 22, 23 and 24, the electron concentration at steady state (represented by an overbar) can be calculated and is given by

$$
\overline{\mathrm{n}}=n_{0}+n_{0}\left[\exp \left(\frac{q \bar{V}}{k_{B} T}\right)-1\right]\left[\cosh \left(\frac{x}{L_{n}}\right)-\operatorname{coth}\left(\frac{L}{L_{n}}\right) \sinh \left(\frac{x}{L_{n}}\right)\right]
$$

where $L_{n}$ is the diffusion length of the electrons. By applying a perturbation to Equations 25 and 26 and applying the Laplace transform, the IS transfer function is obtained as ${ }^{5}$

$$
\mathrm{Z}(\omega)=\left(\frac{R_{W} R_{k}}{1+\frac{i \omega}{\omega_{k}}}\right)^{\frac{1}{2}} \operatorname{coth}\left[\left(\frac{\omega_{k}}{\omega_{d}}\right)^{\frac{1}{2}}\left(1+\frac{i \omega}{\omega_{k}}\right)^{\frac{1}{2}}\right]
$$

where $R_{W}$ is the diffusion resistance, given by

$$
R_{W}=\frac{L}{q A D n_{0} \exp \left(\frac{q V}{k_{B} T}\right)}
$$

$R_{k}$ is the reaction resistance, given by 


$$
R_{k}=\left(\frac{\omega_{d}}{k}\right) R_{W}
$$

$\omega_{d}$ is the characteristic frequency of diffusion, given by

$$
\omega_{d}=\frac{D}{L^{2}}
$$

and $\omega_{k}$ is the characteristic frequency of recombination, given by

$$
\omega_{k}=k
$$
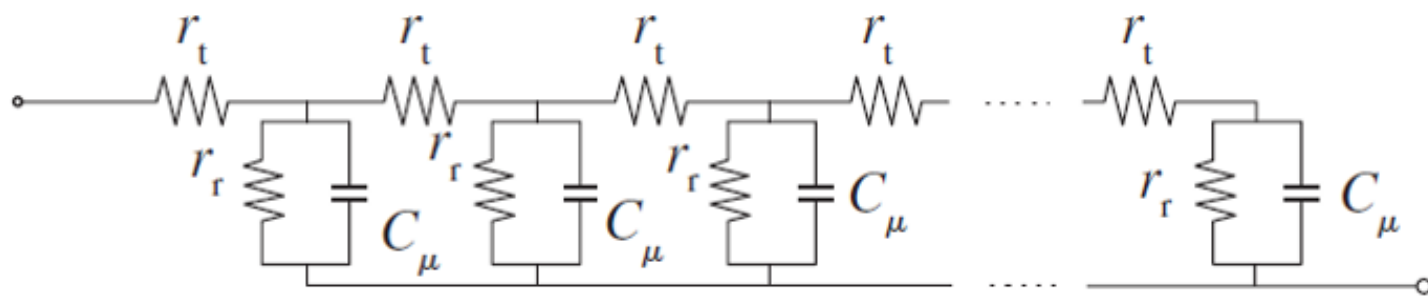

Figure 2.4 Transmission line equivalent circuit for the diffusion-recombination model with a reflecting boundary. $r_{t}$ is the transport resistance per unit length, $r_{r}$ is the recombination resistance per unit length and $C_{\mu}$ is the chemical capacitance. Adapted from Ref. 1 with permission.

The transfer function of Equation 27 is represented in the form of a transmission line EC as shown in Figure 2.4. The elements of the model are given by

$$
\begin{aligned}
& r_{t}=\frac{R_{W}}{L} \\
& r_{r}=L R_{k} \\
& C_{\mu}=\frac{L}{R_{W} D}
\end{aligned}
$$

(a)

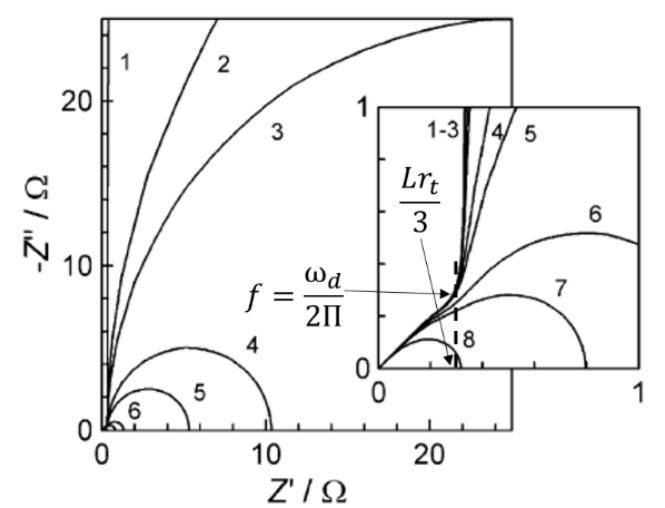

(b)

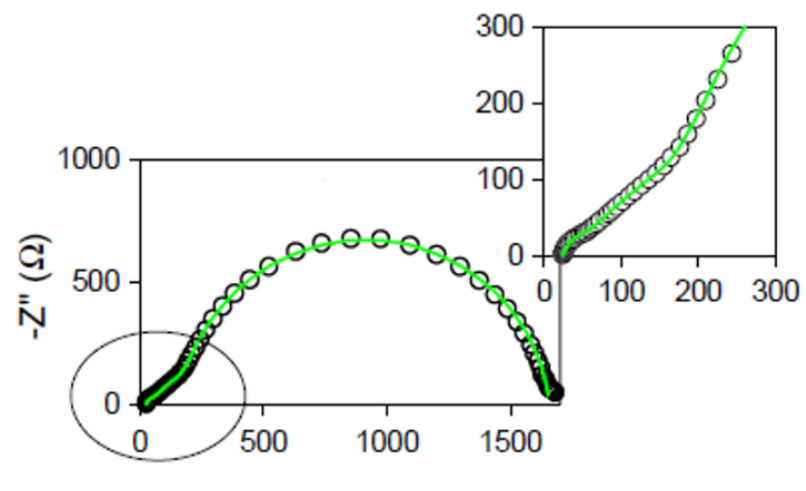

Figure 2.5 (a) Simulations of the diffusion-recombination model impedance of Equation 45 using the transmission line EC of Figure 20. 1-8 varies $r_{r}$ from very high to very low values keeping $r_{t}$ constant. $r_{r}<r_{t}$ for 7-8. (b) Fitting (green line) of measured IS data (circles) of a DSSC with the transmission line model. Adapted from Ref. 6 with permission, Copyright 2004, Elsevier B.V. 
The diffusion-recombination model has been used very successfully to understand, interpret and extract parameters from IS measurements of DSSCs. Simulated IS spectra and an example of measured data fitted to the transmission line are shown in Figure 2.5(a) and 2.5(b) respectively. ${ }^{6}$ At scanning frequencies greater than the characteristic frequency of diffusion $\omega_{d}$, the carriers are unable to diffuse through the semiconductor and reach the collecting electrode to generate a response. This yields a straight line sloped at $45^{\circ}$ to the $\mathrm{x}$-axis in a Z-plane plot. At scanning frequencies lower than $\omega_{d}$, the response depends on the boundary condition. For the reflecting boundary condition as has been discussed, an arc is obtained due to the time constant formed by the parallel combination of $C_{\mu}$ and $r_{r}$, corresponding to filling the density of states of the $\mathrm{TiO}_{2}$ and a finite reaction or recombination resistance that causes the electrons in the conduction band to be lost. Depending on the difference in characteristic frequencies between the diffusion and recombination processes, the response of each is either well differentiated or not, as seen in the inset of Figure 2.5(a).

From the resistance and capacitance values extracted from the fitting and using the time constants of each process, several important physical parameters describing the solar cell transport and recombination properties can be obtained. Figure 2.6(a) shows the different capacitances measured in a DSSC. At low voltages, the capacitance is of the order of tens of $\mu \mathrm{F}$, which is the capacitance of the counter electrode. At intermediate voltages, the typical exponential evolution of the chemical capacitance related to filling of the density of states of $\mathrm{TiO}_{2}$ is observed.
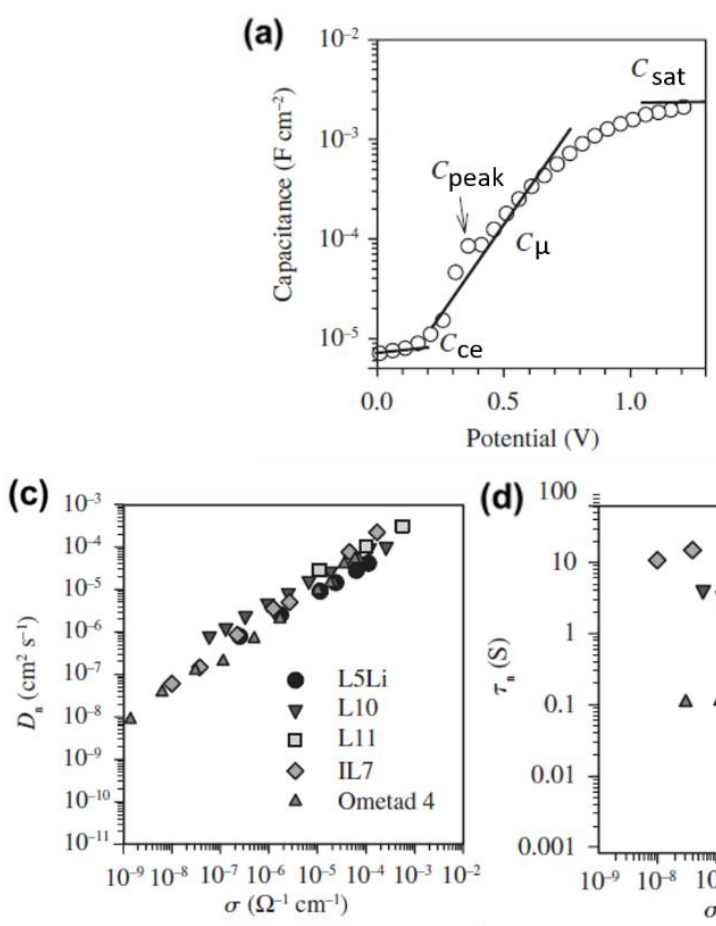
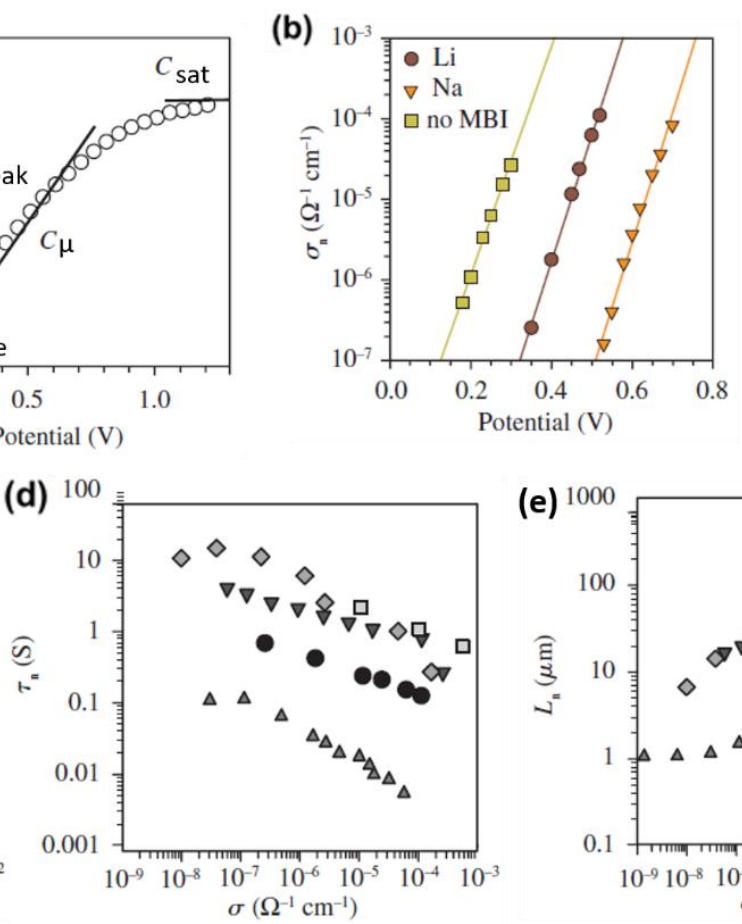

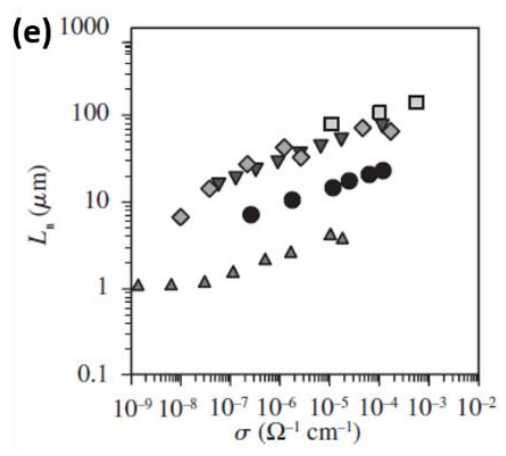

Figure 2.6 Extracted (a) capacitance (b) conductivity (c) diffusion coefficient (d) lifetime and (e) diffusion length of electrons from fitting of IS data with the transmission line EC and their evolution with voltage and conductivity as shown. The legend in (b) corresponds 
to different electrolytes used while the legend for (c, d, e), shown in (c), correspond to different hole selective materials. L stands for liquid electrolyte, IL stands for ionic liquid electrolyte and Ometad is a solid hole conductor. Adapted from Ref. 1 with permission.

A peak in the capacitance can also be observed, which is related to the half occupation of a trap state. ${ }^{7}$ The observation of different capacitances involved in the system indicate that the signal of one capacitance can be masked by that of another depending on their magnitude and whether they are in a parallel or series configuration. Therefore, care must always be taken to distinguish these capacitances by measuring over a large range of voltages. From the transport resistance, the conductivity of the electrons is extracted, shown in Figure 2.6(b). The extracted diffusion coefficient and lifetime values for the electrons (Figure 2.6(c), (d) respectively) yield the diffusion length shown in Figure 2.6(e) according to

$$
L_{n}=\sqrt{D \tau_{\text {rec }}}
$$

Therefore, IS is a very powerful small perturbation technique that provides knowledge of several physical processes occurring on different timescales in the solar cell in a very simplistic and straightforward manner, without the need of expensive and complicated instrumental setups. Furthermore, it simultaneously provides fundamental parameters and constants of processes of recombination, transport and charge storage in a solar cell that can be used to quantify the physics governing these devices.

\subsection{Intensity Modulated Photocurrent Spectroscopy (IMPS)}

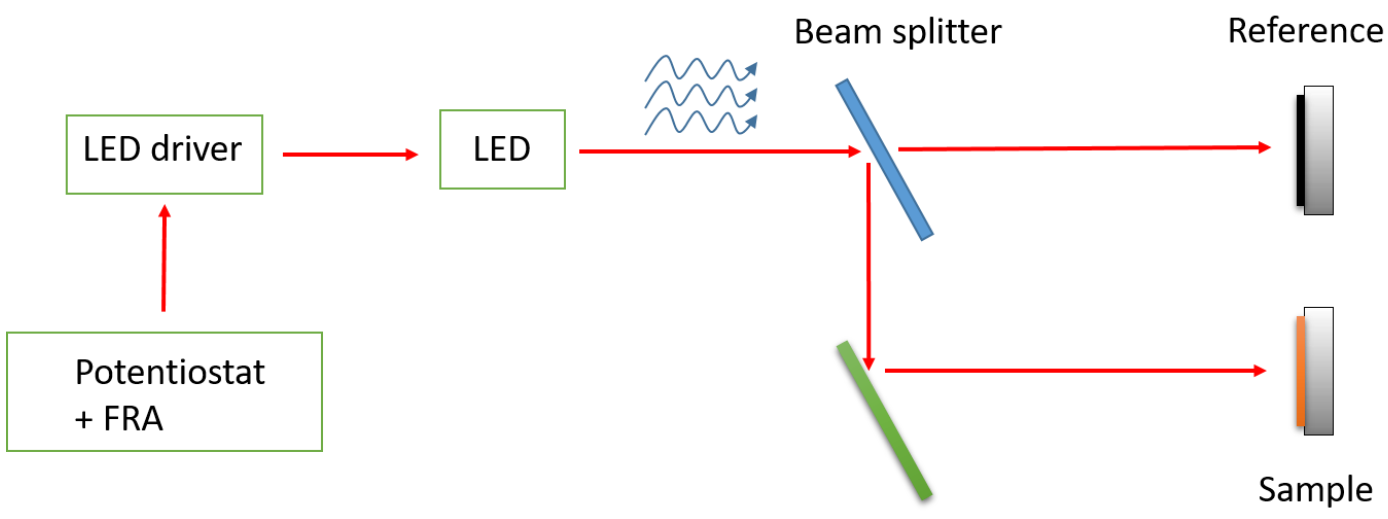

Mirror

Figure 2.7 Illustration of IMPS setup. A potentiostat drives a modulated current to the LED via the LED driver to generate the AC small perturbation of monochromatic light intensity over a given steady state light intensity. This is fed to both a reference and a sample and the modulated current responses are collected to generate the transfer function. In some cases, the light source is calibrated independently without the use of a beam splitter. The frequency modulation is provided using a frequency response analyzer 
(FRA).

This technique involves the measurement of a modulated output current density $\tilde{\jmath}=$ $j_{0} e^{i(\omega t+\varphi)}$ upon application of a small perturbation of input monochromatic photon flux expressed as a current density $\widetilde{J_{\phi}}=j_{\phi, 0} e^{i \omega t}$, superimposed on top of a steady state photon current density $\overline{J_{\phi}}$ that produces a steady state current $\overline{J_{e}} \cdot \varphi$ is the phase difference and $j_{0}$ and $j_{\phi, 0}$ are the amplitudes of the modulated output and input signals respectively. The condition of the perturbation amplitude being much smaller than the steady state photon flux in order to obtain a linear response, as discussed for IS measurements in Section 2.1, must be met. The range of frequencies of the modulated input is usually $1 \mathrm{MHz}-10 \mathrm{mHz}$. Therefore, we have the dimensionless transfer function ${ }^{8}$ ranging from 0 to 1 ,

$$
\mathrm{Q}(\omega)=\frac{\tilde{J}}{\widetilde{J \phi}}
$$

This measurement can be carried out using an external bias and/or a DC background photon current density given by

$$
\overline{J_{\phi}}=q \bar{\phi}
$$

where $\phi$ is the input photon flux. The LF limit of the IMPS transfer function is given by

$$
Q(0)=\frac{d \bar{\jmath}}{d \bar{\jmath} \phi}
$$

It can be seen from Equation 38 that the LF limit is a differential EQE from a given steady state. This quantity is exactly what is calculated in a traditional EQE measurement, where a small perturbation in monochromatic light intensity is applied to the solar cell from a given steady state and the corresponding response is measured, termed the differential spectral response method. ${ }^{9}$ Therefore, IMPS measurements are a reflection of the EQE of the solar cell and hence, measured generally at SC conditions. 
(a)

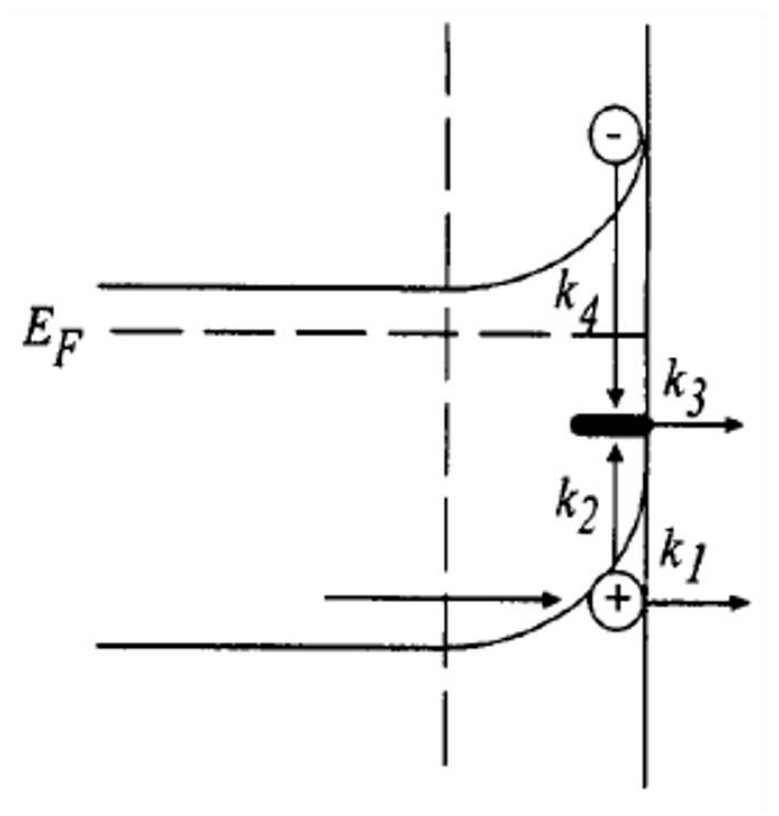

(b)
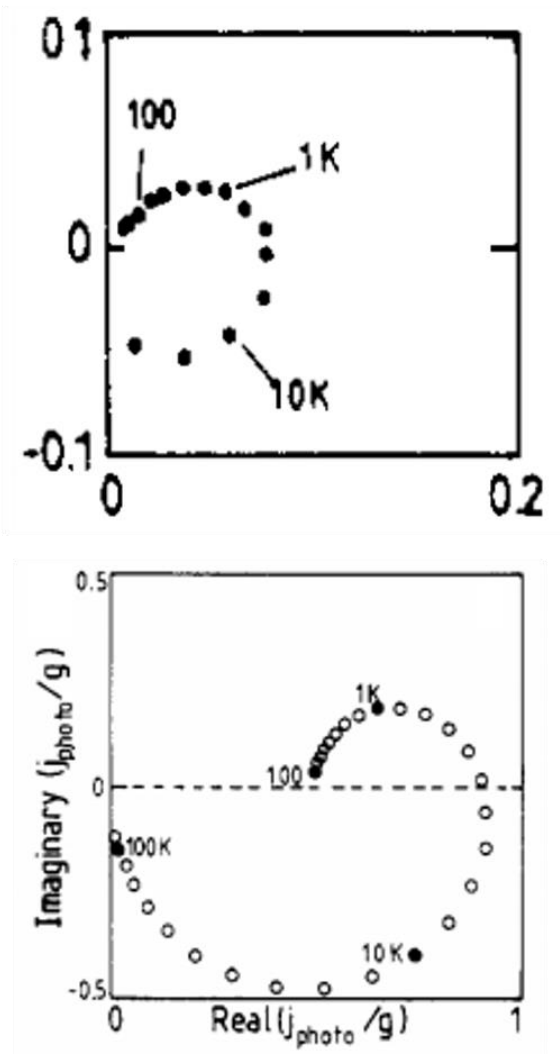

Figure 2.8 (a) Schematic of different processes occurring at the n-type semiconductor/electrolyte interface probed by IMPS and their respective rate constants. $k_{1}$ is the rate of transfer of holes from semiconductor to the electrolyte, $k_{2}$ is the rate of capture of holes in the valence band by a surface state, $k_{3}$ is the transfer of holes from the surface state to the electrolyte and $k_{4}$ is the rate of capture of electrons in the conduction band by a surface state. Dashed lines indicate width of the depletion region. Adapted from Ref. 11 with permission, Copyright 1995, Elsevier B.V. (b) Experimental (top panel) IMPS Q-plane plots of a passive iron electrode in $0.1 \mathrm{M} \mathrm{KOH}$ at $0.5 \mathrm{~V}$ vs $\mathrm{Hg} / \mathrm{HgO}$ with the addition of $\mathrm{K}_{4} \mathrm{Fe}(\mathrm{CN})_{6}$, whose concentration in $\mathrm{mM}$ is shown. Simulated IMPS Qplane plots are shown in the bottom panel based on the processes in (a). The points corresponding to different perturbation frequencies are shown in the plots. Adapted from Ref. 10 with permission, Copyright 1990, American Chemical Society.

IMPS has been used extensively in the study of photoelectrochemical (PEC) systems, involving the decoupling of electron transfer from other processes such as mass transfer and other chemical reactions at a semiconductor/electrolyte interface. ${ }^{10}$ This technique was then adopted to study the properties of the DSSC. The main advantage of using IMPS to study PEC systems is that it is able to provide a small modulation of the minority carrier Fermi level via the modulated photon flux. Therefore, the response provides information directly regarding the minority carriers, which play the dominant role in both photovoltaic and PEC action. This is not the case in IS measurements, which can only modulate the 
majority carrier Fermi level from the semiconductor side via an external modulated voltage and therefore, cannot yield information directly regarding the minority carriers in a PEC system. In addition, light modulated techniques such as IMPS are less destructive on the sample compared to voltage modulated techniques such as IS. A useful strategy is to combine the inferences obtained from both IMPS and IS as they provide the same information in different forms based on the underlying EC, as established by Bertoluzzi and Bisquert. ${ }^{8}$

Figure 2.8(a) shows the different processes involved at a semiconductor/electrolyte interface during an IMPS experiment. An n-type semiconductor is considered, which yields a depletion layer with an upward band bending upon reaching equilibrium with the electrolyte. Note that the majority carrier (electron) Fermi level is controlled by the external contact. Upon the application of a light perturbation, electron-hole pairs are generated. The electrons travel to the collecting electrode while the holes are driven to the semiconductor/electrolyte interface, from where they are transferred to the electrolyte. The sum of the electron and hole currents yields the measured current. In addition to these currents, there are also other processes that occur. Recombination of photogenerated electrons and holes can occur both in the bulk and at the semiconductor/electrolyte interface. This recombination can either occur directly from the conduction band to the valence band, or can involve an intermediary step due to a trap or surface state within the forbidden band gap, which has different rates of electron and hole trapping and release. Further complications can arise from charge transfer of minority holes from the surface state to the electrolyte. The characteristic timescales of these processes shown in Figure 2.8(a) also yield a characteristic response that provides information and de-convolutes these effects from each other, as shown in the Q-plane plot of Figure 2.8(b). A HF arc is obtained whose characteristic frequency is determined by the time constant formed between the series resistance and the geometric or bulk capacitance of the semiconductor electrode. ${ }^{11}$ This occurs because the modulated current is extracted through the series resistance and the bulk capacitance offers a parallel pathway for the current to pass through based on its frequency-dependent impedance. Therefore, their magnitudes determine the net current that is extracted through the circuit and hence the IMPS transfer function at a particular frequency, with a minimum value (0) at very high frequencies related to the capacitor behaving like a short circuit, while at low frequencies, the capacitor is an open circuit and all the current is driven through the series resistance. Therefore, at the lowest frequency of the HF time constant, which is faster than the recombination processes, the maximum photoconversion efficiency is obtained. For lower frequencies, an arc is obtained in the quadrant opposite to the HF arc. The time constant of this LF arc is determined by the competing processes of hole transfer to the electrolyte at the rate $k_{\text {trans }}$ and annihilation of holes by majority carriers through trapping and surface recombination at the rate $k_{r e c} .{ }^{10}$ This is given by

$$
\tau_{L F}=\frac{1}{k_{\text {trans }}+k_{\text {rec }}}
$$

In addition to applications in photoelectrochemistry, IMPS was also used to 
characterize DSSCs. Similar to the theory of IS of DSSCs developed in Section 2.1, the IMPS transfer function was developed by solving the continuity equation, given by ${ }^{12}$

$$
\frac{d n}{d t}=D \frac{d^{2} n}{d x^{2}}-k n+\alpha I_{0} e^{-\alpha x}
$$

where $\alpha$ is the absorption coefficient of the dye molecules, $I_{0}$ is the incident photon flux and $x$ is the position along the length of the $\mathrm{TiO}_{2}$. This Equation is similar to Equation 22, with the addition of the Beer-Lambert expression for absorption of photons by the dye molecules attached along the length of the semiconductor.

(a)

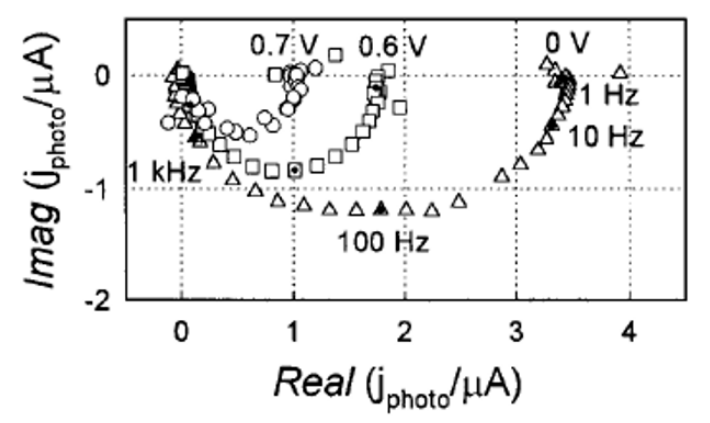

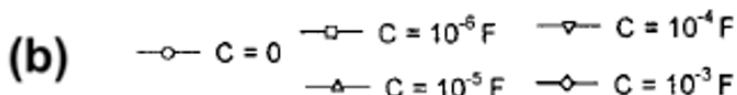

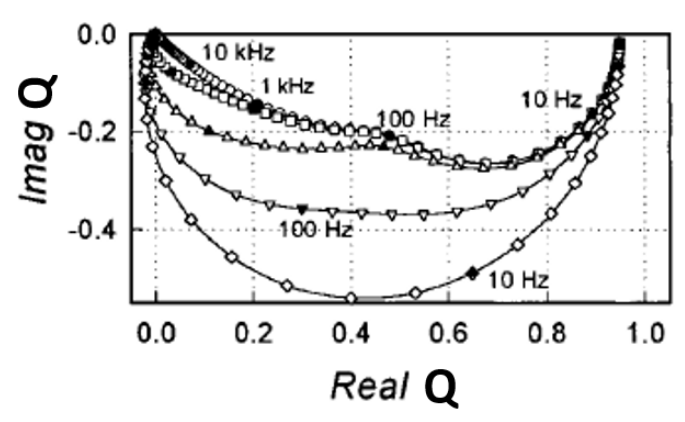

Figure 2.9 (a) Experimental IMPS spectra of a DSSC at different applied potentials as shown and (b) Simulated IMPS response from the diffusion-recombination model for different geometric capacitances of the $\mathrm{TiO}_{2}$ electrode as shown in the legend. Adapted from Ref. 12 with permission, Copyright 1997, American Chemical Society.

By applying a perturbation to Equation 40, using the boundary conditions of Equations 23 and 24, followed by a Laplace transform to the frequency domain, the IMPS transfer function for illumination from the substrate side was calculated as

$$
\begin{aligned}
& \mathrm{Q}(\omega)=\left(\frac{\alpha}{\alpha+\gamma}\right) \frac{e^{\gamma L}-e^{-\gamma L}+2 \alpha \frac{e^{-\alpha L}-e^{-\gamma L}}{\gamma-\alpha}}{e^{\gamma L}-e^{-\gamma L}} \\
& \gamma=\sqrt{\frac{1}{D \tau}+\frac{i \omega}{D}}
\end{aligned}
$$

where $D$ and $\tau$ are the effective diffusion coefficient and effective lifetime of the electrons respectively and $L$ is the thickness of the $\mathrm{TiO}_{2}$ layer. The measured and simulated Q-plane plots of a DSSC is shown in Figure 2.9. At high frequencies, greater than the characteristic frequency of diffusion of electrons, the diffusion of the electrons is observed in the form of a straight line sloped at $45^{\circ}$ to the x-axis, followed by an arc related to the $R_{s} C_{g}$ attenuation. Depending on the characteristic frequency of this process, the characteristic signal of diffusion of the electrons can be masked, as shown for different values of the electrode capacitance in Figure 2.9(b). By obtaining $\tau$ from Intensity Modulated Photovoltage Spectroscopy (IMVS), the value was used in Equation 41 to fit the experimental IMPS Q-plane plots and extract the effective diffusion coefficient values. As is shown in Figure 2.10, both the effective diffusion coefficient and effective lifetime 
for electrons evolve with light intensity, due to the action of trapping and de-trapping in the density of states within the bandgap of the $\mathrm{TiO}_{2}$. However, the square root of the product of the two quantities is the diffusion length, which has a very weak dependence on light intensity. ${ }^{13}$ This was explained by Bisquert and Vikhrenko using a multipletrapping model, ${ }^{14}$ where the trapping and de-trapping of free electronic carriers to and from localized states inside the bandgap play a role in the kinetic measurements of diffusion coefficient and lifetime, but do not affect steady state quantities such as the diffusion length and conductivity.

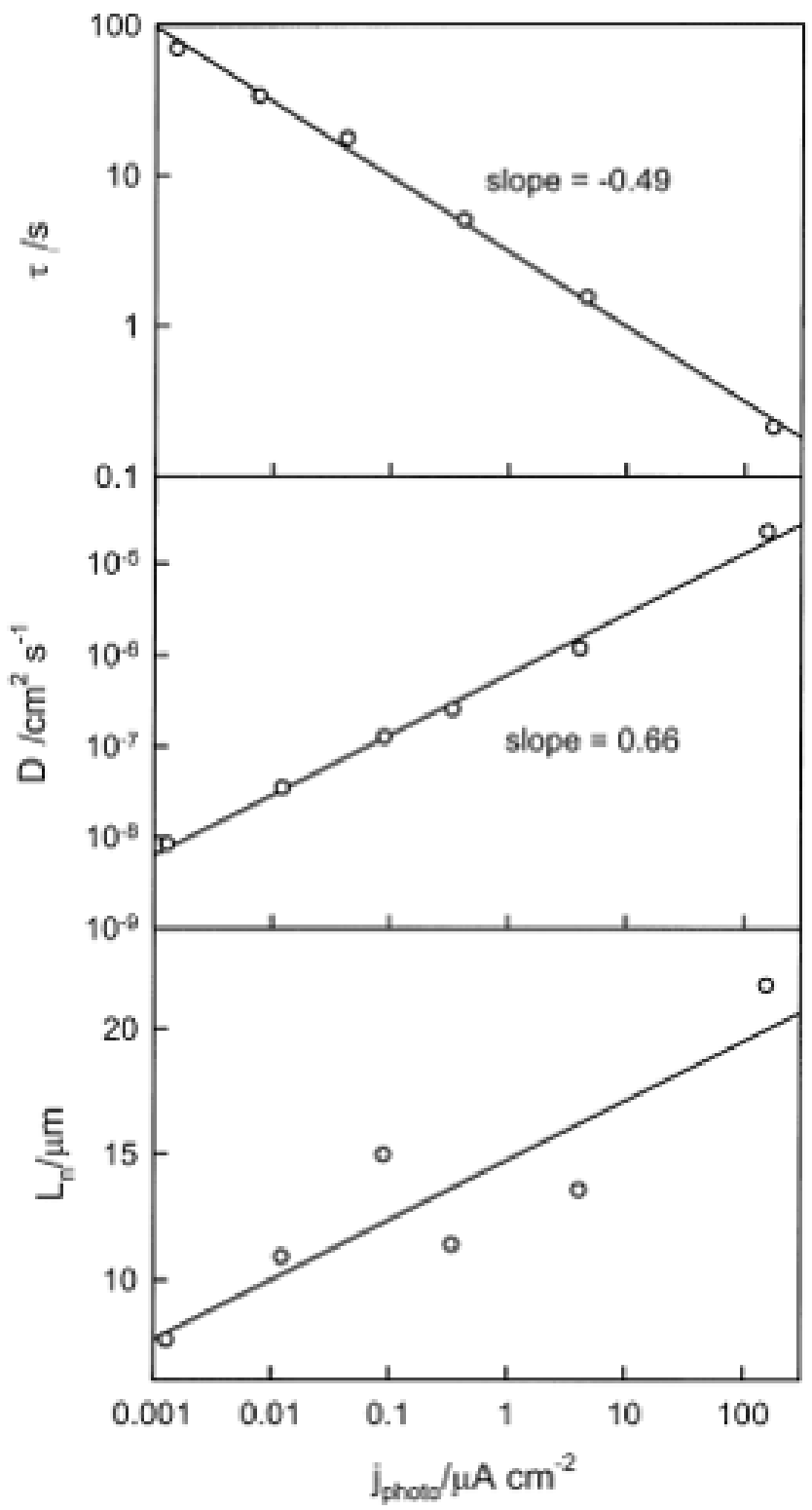

Figure 2.10 Dependence of effective electron lifetime $\tau$, effective electron diffusion coefficient $D$ and extracted electron diffusion length $L_{n}$ on DC short circuit photocurrent density (light intensity) for a DSSC. Adapted from Ref. 13 with permission, Copyright 1999, Elsevier B.V. 


\subsubsection{Measurement protocols}

The IMPS measurements carried out in this thesis were made using a blue LED, emitting at $470 \mathrm{~nm}$. The DC light intensity corresponding to the input DC current from the LED driver was calculated using a reference Si photodiode. The transfer function calculated by the software is the ratio of the modulated extracted current and the modulated input current to the LED that produces the modulated light intensity. Therefore, a factor needs to be introduced in the transfer function that converts the modulated input current to the LED to the modulated current density of input photons. This is carried out by:

1) Calculation of the DC light intensity for different input currents to the LED using the calibrated Si reference photodiode.

2) Interpolation of the DC light intensity versus input LED current plot is carried out. For the required DC light intensities for the IMPS measurement, the required amplitude of the input LED current that produces a $10 \%$ modulation of the DC light intensity is calculated. These values are then introduced into the FRA software that creates the current perturbation to the LED.

3) The slope $k$ of the light intensity $(I)$ vs LED current $\left(j_{L E D}\right)-d j_{L E D} / d I$ is calculated and the transfer function in the software is multiplied by $(h c k / q \lambda)$, where $h$ is Planck's constant, $c$ is the speed of light, $\lambda$ is the wavelength of the input photons. Dividing this value by the area of illumination yields the IMPS transfer function $Q$ defined in Equation 36. 


\subsection{References}

1. Bisquert, J.; Fabregat-Santiago, F. Impedance spectroscopy: a general introduction and application to dye-sensitized solar cells 2010.

2. Zaban, A.; Greenshtein, M.; Bisquert, J. Determination of the electron lifetime in nanocrystalline dye solar cells by open-circuit voltage decay measurements. ChemPhysChem 2003, 4 (8), 859-864.

3. Zaban, A.; Meier, A.; Gregg, B. A. Electric Potential Distribution and Short-Range Screening in Nanoporous $\mathrm{TiO}_{2}$ Electrodes. The Journal of Physical Chemistry B 1997, 101 (40), 7985-7990.

4. Soedergren, S.; Hagfeldt, A.; Olsson, J.; Lindquist, S.-E. Theoretical Models for the Action Spectrum and the Current-Voltage Characteristics of Microporous Semiconductor Films in Photoelectrochemical Cells. The Journal of Physical Chemistry 1994, 98 (21), 5552-5556.

5. Bisquert, J. Theory of the impedance of electron diffusion and recombination in a thin layer. The Journal of Physical Chemistry B 2002, 106 (2), 325-333.

6. Fabregat-Santiago, F.; Bisquert, J.; Garcia-Belmonte, G.; Boschloo, G.; Hagfeldt, A. Influence of electrolyte in transport and recombination in dye-sensitized solar cells studied by impedance spectroscopy. Solar Energy Materials and Solar Cells 2005, 87 (14), 117-131.

7. Bisquert, J. Theory of the impedance of charge transfer via surface states in dyesensitized solar cells. Journal of Electroanalytical Chemistry 2010, 646 (1-2), 43-51.

8. Bertoluzzi, L.; Bisquert, J. Investigating the consistency of models for water splitting systems by light and voltage modulated techniques. The Journal of Physical Chemistry Letters 2016, 8 (1), 172-180.

9. Cowan, S. R.; Wang, J.; Yi, J.; Lee, Y.-J.; Olson, D. C.; Hsu, J. W. Intensity and wavelength dependence of bimolecular recombination in P3HT: PCBM solar cells: A white-light biased external quantum efficiency study. Journal of Applied Physics 2013, 113 (15), 154504.

10. Peter, L. M. Dynamic aspects of semiconductor photoelectrochemistry. Chemical Reviews 1990, 90 (5), 753-769.

11. Ponomarev, E.; Peter, L. A generalized theory of intensity modulated photocurrent spectroscopy (IMPS). Journal of Electroanalytical Chemistry 1995, 396 (1-2), 219-226.

12. Dloczik, L.; Ileperuma, O.; Lauermann, I.; Peter, L. M.; Ponomarev, E. A.; Redmond, G.; Shaw, N. J.; Uhlendorf, I. Dynamic Response of Dye-Sensitized Nanocrystalline Solar Cells: Characterization by Intensity-Modulated Photocurrent Spectroscopy. The Journal of Physical Chemistry B 1997, 101 (49), 10281-10289.

13. Peter, L. M.; Wijayantha, K. G. U. Intensity dependence of the electron diffusion 
length in dye-sensitised nanocrystalline $\mathrm{TiO}_{2}$ photovoltaic cells. Electrochemistry Communications 1999, 1 (12), 576-580.

14. Bisquert, J.; Vikhrenko, V. S. Interpretation of the time constants measured by kinetic techniques in nanostructured semiconductor electrodes and dye-sensitized solar cells. The Journal of Physical Chemistry B 2004, 108 (7), 2313-2322. 


\section{Chapter 3}

\section{Critical Overview}

\subsection{Goals and strategies}

Perovskite solar cells (PSCs) have shown a rapid improvement in performance, currently in excess of $20 \%$, since their inception in 2009. These high efficiencies, coupled with ease of production using simple solution-processed methods and large tunability in properties, is generating great interest in the PSC as a viable addition to the devices that will satiate the huge projected demand for clear and renewable energy in the near future.

However, a major impediment to the commercialization of the PSC is its low tolerance to relative humidity and ambient environmental conditions, showing deterioration in performance over timescales ranging from a few hours to several months, depending on materials and methods. ${ }^{1-2}$ This mechanism is reflected in the hysteresis observed in the current-voltage $(j-V)$ curve of a PSC, which is deeply connected to the mixed ionicelectronic conduction in PSCs, where charged lattice defects or ions hop along the crystal lattice. ${ }^{3}$ This hysteresis depends strongly on measurement conditions such as biasing voltage, scan direction, scan speed and light intensity preconditioning. ${ }^{4-5}$ This leads to erroneous estimation of the stabilized power output of the PSC, which is a critical parameter in its description. In order to understand the mechanisms driving this effect, several device models have been proposed that involve modelling the PSC as a n-i-p heterojunction. ${ }^{6}$ This involves assuming an intrinsic perovskite absorber with a constant built-in electrical field which is responsible for photocarrier collection, established by the difference in workfunctions of the selective contacts. The ions drift, shield and modulate this electrical field depending on external measurement conditions. This charge collection model, in conjunction with trapping and de-trapping of electronic carriers, has been used to explain and reproduce some of the hysteretic trends observed in PSCs. ${ }^{7}$ However, these models are unable to explain several of the unique physical mechanisms observed in the PSCs, such as the apparently unlimited large LF capacitance of the order of milliFarads $(\mathrm{mF}),{ }^{8}$ extremely slow recombination timescales in the order of seconds ${ }^{9}$ and a strong dependence of these parameters and hence the performance on the selective contact materials. ${ }^{10}$ Furthermore, the role of charge extraction at the interfaces is either neglected or unclear, which is modulated strongly by the potential drops created by the ions at a given steady state. In addition, there is strong evidence of very large diffusion lengths of the order of micrometres $(\mu \mathrm{m})$ in PSCs, ${ }^{11-12}$ indicating that the role of a built-in electrical field in transport, charge collection and ultimately obtained $V_{o c}$ should be minor. Therefore, there is an urgent need to develop a PSC device model that incorporates all of these features while reproducing its unique hysteretic behaviour. In summary, the main goal of this thesis is:

Development of a robust device model and equivalent circuit of the perovskite 
solar cell that can reproduce its unique response and hysteretic behaviour that is aligned with the physical inferences obtained from several other techniques, irrespective of materials and methods, thereby leading to an understanding of its operating mechanisms to direct further material modifications.

In order to achieve this goal, the following questions need to be explored:

- How do ions affect the relationship between the internal and external voltage of the PSC in a kinetic measurement?

- How is recombination, capacitance and charge extraction affected by ions at the selective contact/perovskite interface?

- What is the role of a built-in electrical field in the transport of photogenerated charges in a PSC? Do the selective contact workfunctions limit the maximum $V_{o c}$ obtained?

- Can the proposed EC explain the response of the PSC from another small perturbation technique, since the underlying circuit is the same?

The answers to these questions and the subsequent development of this model can help understand regions that store charge preferentially, such as the interfaces between the perovskite and the selective contacts, which can serve as strong recombination centers. These interfaces can then be passivated suitably to improve performance of the PSC. Identifying the importance of the built-in electrical field for transport of photogenerated carriers in the PSC will allow for understanding the dependence of the $V_{o c}$ on the contact workfunctions, thereby allowing or blocking a range of materials that can be explored as selective contacts. Finally, understanding the charge extraction at the perovskite/selective contact interface that is strongly modulated by the potential drops created by the ions, will provide an improved understanding of the interfacial kinetics that limit the performance of the PSC.

In order to achieve these goals, variations in device structure and formulation are carried out as per requirement, which is explained in the subsequent paragraphs. The main methods used to probe the recombination, charge storage and transport properties are Impedance Spectroscopy (IS) and Intensity Modulated Photocurrent Spectroscopy (IMPS), which are small perturbation methods that obtain information through measurement of the response of the PSC to an input modulated voltage and photon flux respectively. Further details of these techniques are provided in Chapter 2. Simulations and numerical methods were carried out using MATLAB and/or Wolfram Mathematica.

\subsection{Interfacial polarization}

Based on the stated goals, an initial model termed the surface polarization model (SPM) was developed to explain the various hysteresis patterns observed in kinetic measurements, specifically the $j-V$ curve of a PSC. This model assumes a p-type MAPI absorber with dopant density $p_{0}$ and involves the formation of an electrostatic potential 
$V_{s}$ at the ESL/MAPI perovskite interface at large steady state forward bias (close to $\left.V_{o c}\right){ }^{8}$ This potential is reflected in the upward band bending at this interface due to the arrival of positively charged ions under large injection or illumination conditions. This upward band bending can enter the majority carrier (hole) Fermi level, creating a huge density of both accumulated ionic and electronic holes $p_{s}$ at this interface, as has also been measured using Scanning force microscopy (SFM) measurements on PSCs under illumination. This is shown in Figure 3.1. ${ }^{13}$ Upon cycling the external voltage from this state, the response of the internal voltage, which is generally extremely fast in conventional solar cells, is delayed in the PSC due to the ions that drift away from the interface at a slow rate. The slow diffusion of the ions leaves a density of electronic charge remaining at the interface, which can either be extracted or can subsequently recombine at the interface. It is this density of electronic charge that yields the very slow evolution of the $V_{o c}$ in open-circuit voltage decay measurements, as the contacts measure the electronic Fermi levels only.

(a)

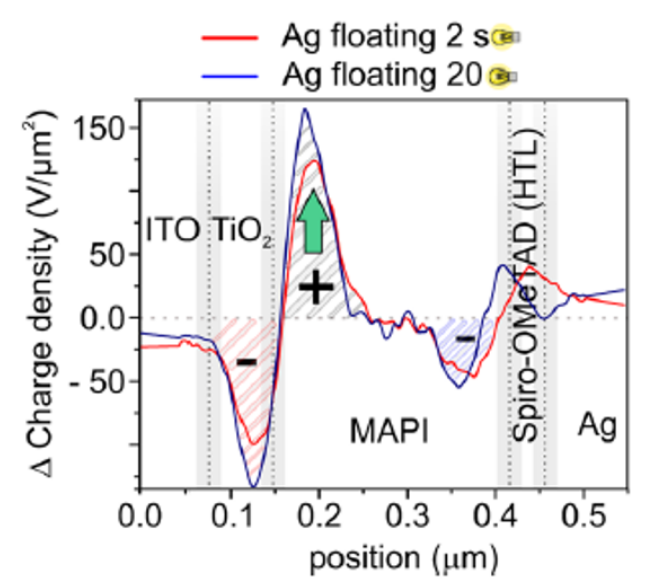

(b)

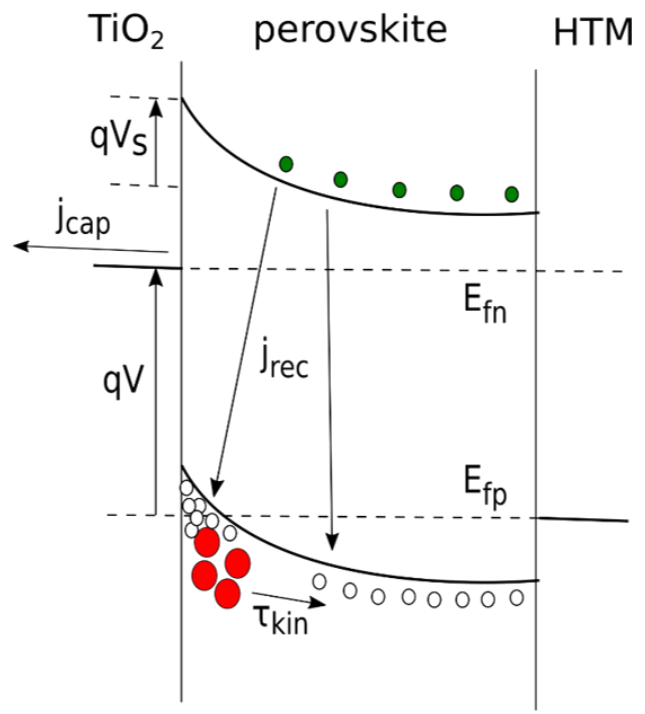

Figure 3.1 (a) Charge densities calculated at OC conditions under illumination from scanning force microscopy measurements on MAPI solar cells with the Ag electrode floating. Note the unbalanced, excess positive charge density at the $\mathrm{TiO}_{2} / \mathrm{MAPI}$ interface that increases with illumination time. Adapted with permission from Ref. 13, Copyright 2016, American Chemical Society. (b) Schematic of the surface polarization model with $V$ being the external potential and and $V_{S}$ being the electrostatic potential due to ionic accumulation at the interface respectively. $E_{f n}$ and $E_{f p}$ are the electron and hole Fermi levels respectively, which are assumed to be flat because of a large diffusion coefficient. $\tau_{\text {kin }}$ is the timescale of drift of ions away from the interface. Electrons are shown as green spheres, holes as white spheres and cations as red spheres.

Therefore, the connection between the internal and external voltage of the PSC at steady state (overbar) can be defined as 


$$
\bar{V}_{s}=\bar{V}-V_{b i}
$$

where $V_{b i}$ is the built-in potential at equilibrium. Upon cycling the voltage from a steady state condition, the evolution of the surface voltage is given by

$$
\frac{d V_{s}}{d t}=\frac{V_{s}-\left(V-V_{b i}\right)}{\tau_{k i n}}
$$

where $\tau_{k i n}$ is the timescale of ionic drift away from the interface. The excess charge density at the interface is

$$
Q_{s}=q p_{s}=Q_{s 0} \exp \left(q V_{s} / \gamma k_{B} T\right)
$$

yielding the accumulation capacitance

$$
C_{a c c}=\frac{d Q_{s}}{d V_{s}}=\left(\frac{q Q_{s 0}}{\gamma k_{B} T}\right) \exp \left(q V_{s} / \gamma k_{B} T\right)
$$

Note that the ideal value of $\gamma$ is 2 . The discharge of the accumulated holes yields a capacitive current given by

$$
j_{c a p}=\frac{d Q_{s}}{d t}=C_{a c c} \frac{d V_{s}}{d t}
$$

A recombination current is created by the recombination of the accumulated holes at the interface and the bulk holes with injected electrons, given by

$$
j_{\text {rec }}=k_{\text {rec }} n Q_{s}=j_{\text {rec } 0} \exp \left(q V_{b i} / \gamma k_{B} T\right) \exp \left[q\left(V_{s} / \gamma+V / \beta\right) / k_{B} T\right]
$$

Here, $j_{\text {rec } 0}$ is the reverse saturation current density and $\beta$ is the ideality of recombination. The equations 47 and 48 are then superimposed on the photogenerated current as

$$
\mathrm{j}=\overline{J_{p h}}-j_{\text {rec } 0} \exp \left[\frac{q\left(V_{s} / \gamma+V / \beta+V_{b i} / \gamma\right)}{k_{B} T}\right]+C_{a c c} \frac{d V_{S}}{d t}
$$

At steady state, Equation 49 breaks down into the standard diode form given by

$$
\overline{\mathrm{J}}=\overline{J_{p h}}-j_{\text {rec } 0} \exp \left[\frac{q \overline{\mathrm{v}}}{m k_{B} T}\right]
$$

where

$$
\mathrm{m}=\frac{\beta \gamma}{\beta+\gamma}
$$

The SPM is able to reproduce several of the hysteretic trends and their strong dependence on measurement parameters for PSCs, shown in Figure 3.2, especially the 'bump' in the $j-V$ curve close to $V_{o c}$, capacitive behaviour with respect to scan rate and the apparent shunt resistance effect described in Figure 1.7(a),(b). It is also able to reproduce the case where the apparent $j_{s c}$ is enhanced, due to very slow discharge of the accumulated carriers as seen in Figure 1.7(c). In addition, its features allow the explanation of the huge capacitances observed for PSCs, the extremely slow $V_{o c}$ decay and strong dependence of hysteresis and capacitance on the ESL material. It must be noted that this model is a recombination-based model as transport of photocarriers is assumed to be excellent through the PSC, with the main governing factor being the interface. 
(a)

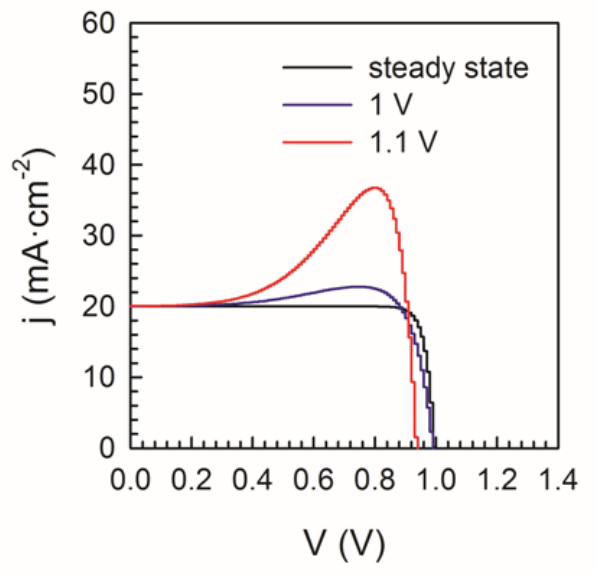

(c)

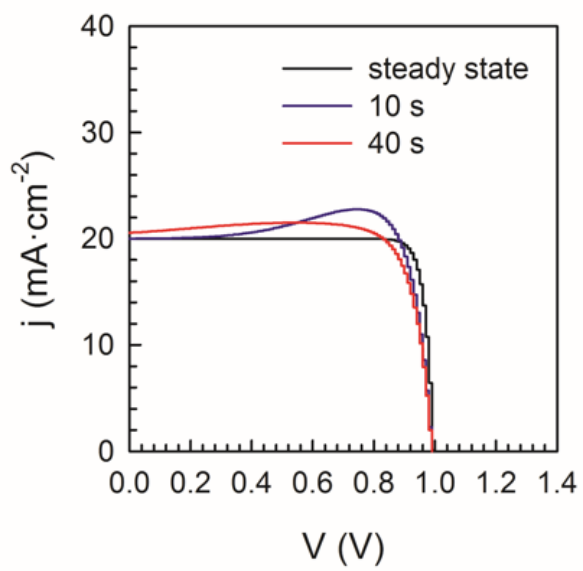

(b)

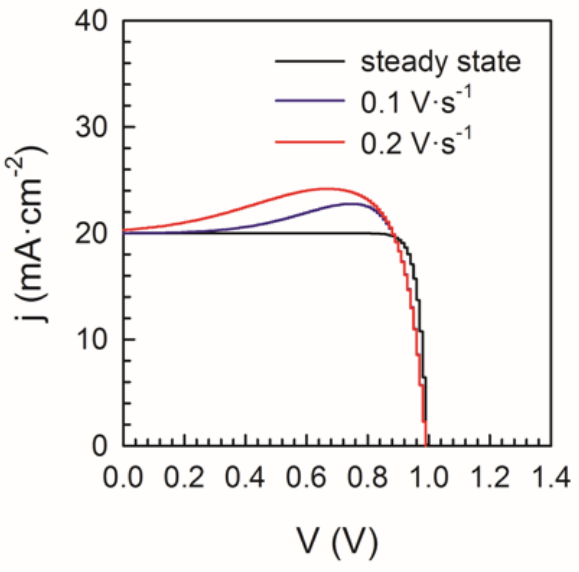

(d)

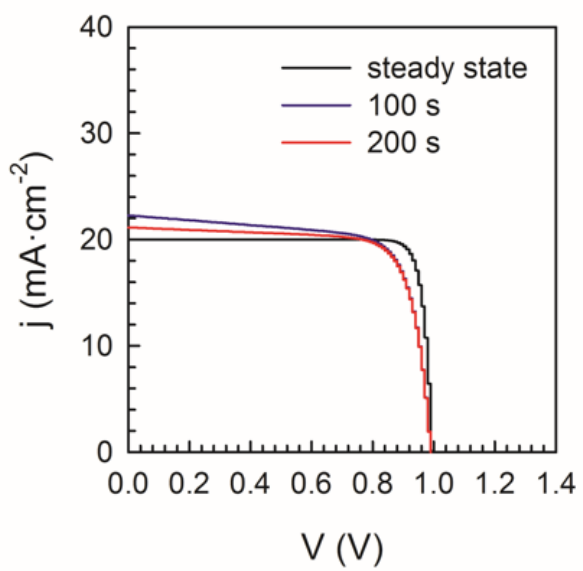

Figure 3.2 (a) Effect of pre-bias voltage (b) scan rate (c, d) small and large $\tau_{\text {kin }}$ values respectively, simulated from the SPM. Parameters used were: $Q_{s 0}=4.4 \times 10^{-8} \mathrm{C} \cdot \mathrm{cm}^{-2}, b=$ $100 \mathrm{mV} \cdot \mathrm{s}^{-1}, V_{b i}=350 \mathrm{mV}, \beta=2, \gamma=2, j_{p h}=20 \mathrm{~mA} \cdot \mathrm{cm}^{-2}, \mathrm{j}_{\mathrm{rec} 0}=4.8 \times 10^{-16} \mathrm{~mA} \cdot \mathrm{cm}^{-2}, \tau_{k i n}$ $=10 \mathrm{~s}$ for $(\mathrm{a}),(\mathrm{b})$.

\subsection{ESL/perovskite interface recombination}

However, a question arises - How does the perovskite sustain such large densities of charge at the $\mathrm{TiO}_{2} /$ perovskite interface at steady state? It then becomes apparent that an extra recombination pathway must be considered, between the accumulated holes in the perovskite side and the corresponding electrons in the $\mathrm{TiO}_{2}$ side, especially in the case of voltage-based hysteresis. This is exactly what was observed in the unique hysteretic trends of stable triple layer mesoscopic PSCs, whose structure is shown in Figure 3.3(a). These cells showed variations in only the $V_{o c}$, with $j_{s c}$ and FF values very similar, as seen in Figure 3.3(c). Furthermore, the nature of the hysteresis could be tuned depending on the thickness of the compact $\mathrm{TiO}_{2}$ layer, with a change from normal hysteresis to inverted hysteresis (as defined in Section 1.2.3.2.1) with reducing thickness of the $\mathrm{c}-\mathrm{TiO}_{2}$ layer. In this case, it becomes very difficult to explain the hysteretic trends using charge collection 
models since the currents are almost identical in all cases, with variations in only the recombination properties that affect the $V_{o c}$.

(a)

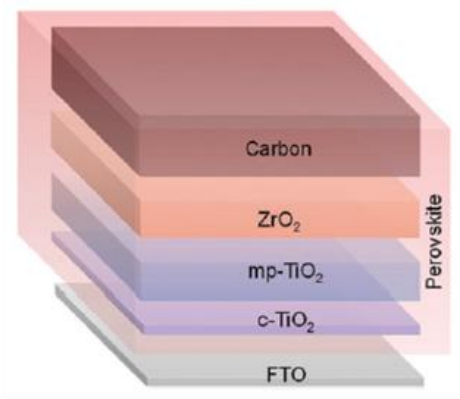

(b)

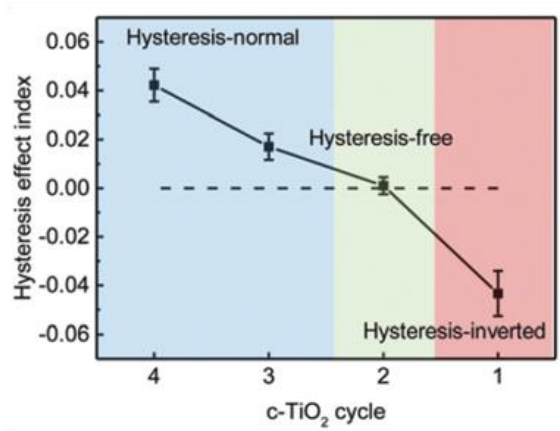

(c)
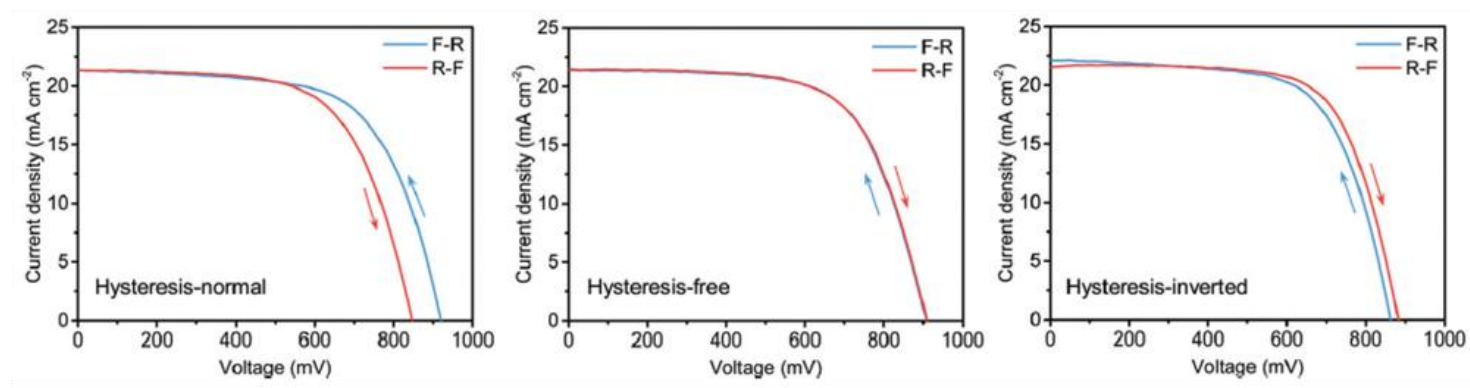

(d)

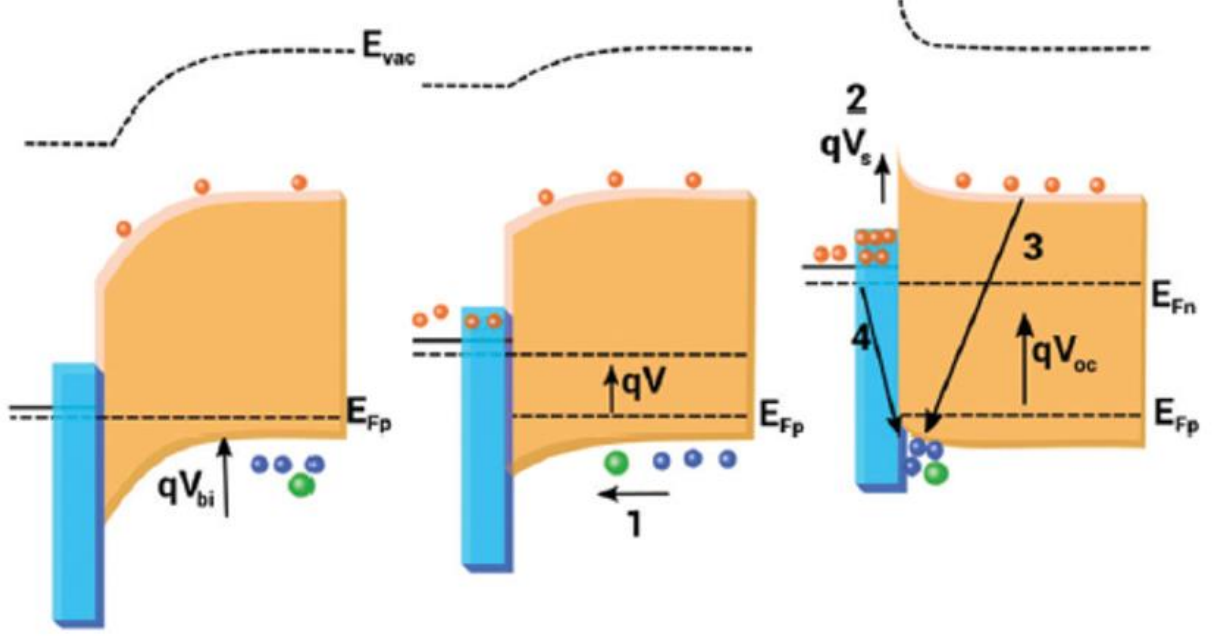

Figure 3.3 (a) Device structure of triple layer mesoscopic PSCs (b) variation of nature of hysteresis depending upon number of cycles of spray-coated c- $\mathrm{TiO}_{2}$ layer (c) $j-V$ curves of devices with corresponding $\mathrm{c}-\mathrm{TiO}_{2}$ layer thicknesses shown in (b), yielding a unique hysteresis only in $V_{o c}$ values. F-R indicates forward to reverse bias scan and vice versa for R-F scan. (d) Schematic of different processes occurring at the ESL (blue)/perovskite (brown) interface in a kinetic measurement. Upon the application of a forward bias, holes (purple) and cations (green) are brought towards the ESL/perovskite interface (process 1) by the injected electrons (orange) from the ESL side. At large forward biases, accumulation of holes and cations leads to a surface electrostatic potential $V_{s}$, causing the 
bands to bend upward at the perovskite side (process 2). The steady state voltage obtained is determined by the recombination of the accumulated holes with electrons in the perovskite bulk (process 3) and electrons in the ESL side (process 4).

Therefore, the SPM was extended in order to account for this phenomenon. Briefly, as described previously, the model considers the accumulation of a large density of cations and holes at the ESL/perovkite interface at large forward (F) bias, which is almost a steady state value and hence does not depend on the kinetics of the ions. This yields a $V_{o c}$ that is reduced in a reverse to forward (RF) bias scan due to the slow kinetics of the ions, which take time to arrive at the ESL/perovskite interface and create accumulation of holes to increase the $V_{o c}$. This causes the 'normal' hysteresis shown in Figure 3.3(c), where the $V_{o c}$ of the FR scan is larger than the RF scan. However, reducing the thickness of the $\mathrm{c}-\mathrm{TiO}_{2}$ layer opens up an extra recombination pathway between the accumulated holes on the perovskite side and the corresponding accumulated electrons on the $\mathrm{TiO}_{2}$ side. This recombination reduces the net $V_{o c}$ obtained at steady state $\mathrm{F}$ voltages while not being significant during an RF scan due to the slow accumulation kinetics. This means that for smaller thicknesses of the c- $\mathrm{TiO}_{2}$ layer, the $V_{o c}$ in an FR scan is reduced, while the $V_{o c}$ of the RF scan is unaffected, yielding the hysteresis patterns of Figure 3.3(b) and 3.3(c). Therefore, by qualitatively accounting for surface recombination of accumulated holes with the electrons in the $\mathrm{c}-\mathrm{TiO}_{2}$ layer, the interplay between surface recombination and accumulation explained the variation from normal to inverted $V_{o c}$ hysteresis in these PSCs. 


\subsection{Role of the built-in electrical field}

(a)

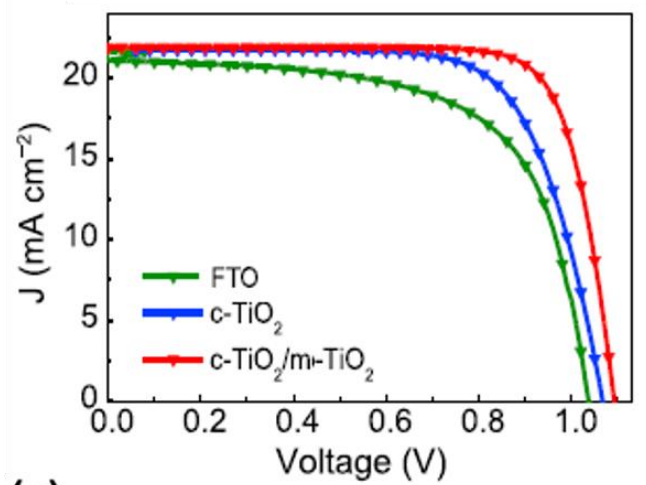

(c)

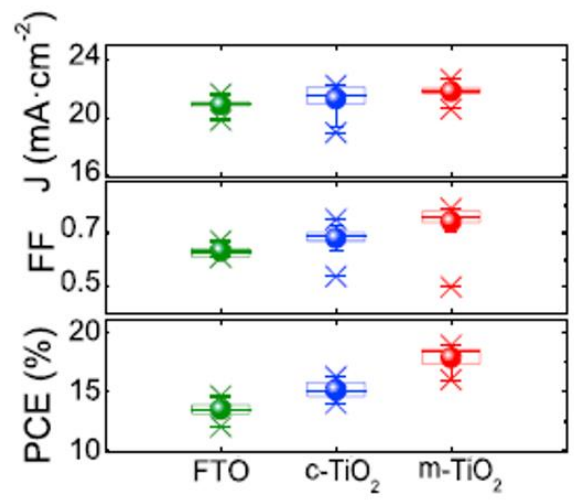

(b)

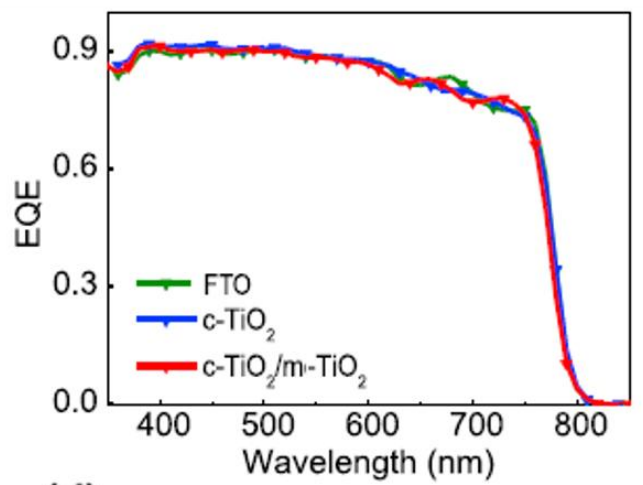

(d)

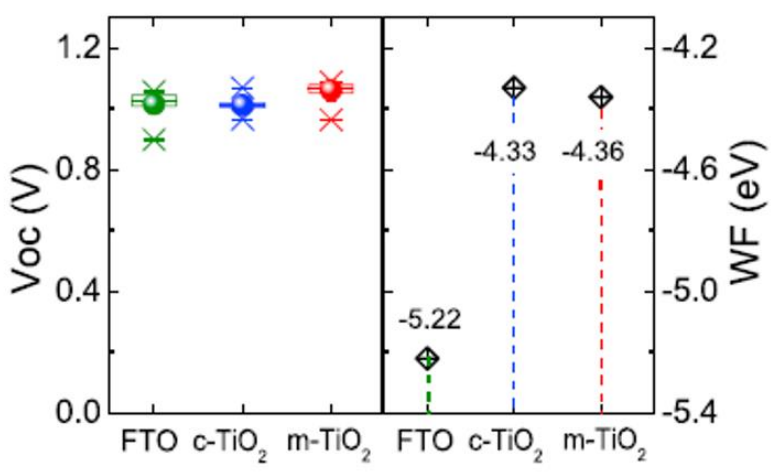

Figure 3.4 (a) $j-V$ curves of MAPI perovskite solar cells with the configuration ESL/perovskite/Spiro-OMeTAD/Au, with different ESLs as shown in legend. (b) External quantum efficiency of the different measured cells as shown in legend. (c) Statistics of the short circuit current density, fill factor (FF) and photoconversion efficiency (PCE) of cells with different ESLs as shown. (d) Obtained $V_{o c}$ and corresponding workfunction values of cells with different ESLs.

After establishing a robust recombination-based kinetic model for PSCs, a deeper understanding of the nature of transport in a PSC was required due to the fact that PSCs show large variations in the photocurrent depending on measurement conditions even though reported diffusion lengths are very large (in the order of $\mu \mathrm{m}$ ), several times larger than the perovskite film thickness. Further development and validation of the model required an understanding of the equilibrium potential distribution in the PSC, to identify if the potential drop due to contact workfunction (WF) difference is absorbed at the interfaces in a small layer or leads to a built-in electrical field through the whole absorber layer. This distribution of the equilibrium potential can have strong implications on the nature of transport of photogenerated carriers and the obtained $V_{o c}$. Therefore, a systematic study of the $V_{o c}$ values was carried out on high efficiency $\mathrm{CH}_{3} \mathrm{NH}_{3} \mathrm{PbI}_{3}$ (MAPI) cells by selective removal and/or variation of the different layers constituting the ESL. As seen from Figure 3.4(a), (b) and (c), the only differentiating factor in the performance of 
the cells was the variation in $\mathrm{FF}$, with $j_{s c}$ and $V_{o c}$ showing very little change. From ultraviolet photoelectron spectroscopy (UPS) measurements, the WF of the different ESLs was measured. As seen from Figure 3.4(d), an almost $1 \mathrm{eV}$ variation in the WF from FTO to $\mathrm{m}_{-} \mathrm{TiO}_{2}$ yielded a change in $V_{o c}$ of less than $100 \mathrm{mV}$. These observations have significant ramifications on the understanding of the origin of the photovoltage in PSCs and their relation to the selective contacts. As described in Section 1.2.3.4 and shown in Figure 3.5(a) and (b), the PSC is frequently modelled as an intrinsic absorber with a builtin electrical field at equilibrium conditions through the absorber that is generated by the difference in WF of the two contacts. This creates a favourable condition for the separation and extraction of photogenerated carriers, with the electrons and holes driven by the electrical field to the ESL and HSL respectively. Upon applying a forward bias, the tilting of the bands is reduced, reducing the driving force for carrier separation, Figure 3.5(c). Therefore, in such a model, the maximum $V_{o c}$ obtained is equal to or slightly larger than the $V_{b i}$ and the WF difference between the ESL and HSL becomes critical in determining the photovoltage. ${ }^{6}$ However, it can be seen that there is no correlation between the WF of the ESL and the $V_{o c}$ obtained for high efficiency MAPI PSCs. This indicates firstly that the $V_{b i}$ due to equilibration between the perovskite absorber and the selective contacts occurs in a small layer close to the interfaces, with a bulk electrical field-free region. Secondly, it also indicates that the perovskite is very efficient at separating and transporting photogenerated carriers without the requirement of an electric field within the absorber. Therefore, further developments in high efficiency PSCs will require tuning of the interfacial recombination and extraction characteristics rather than focussing on improving charge collection. A wide range of alternate materials can be explored based on the fact that the $V_{o c}$ obtained in the PSC is independent of the WF of the contacts. 
(a)

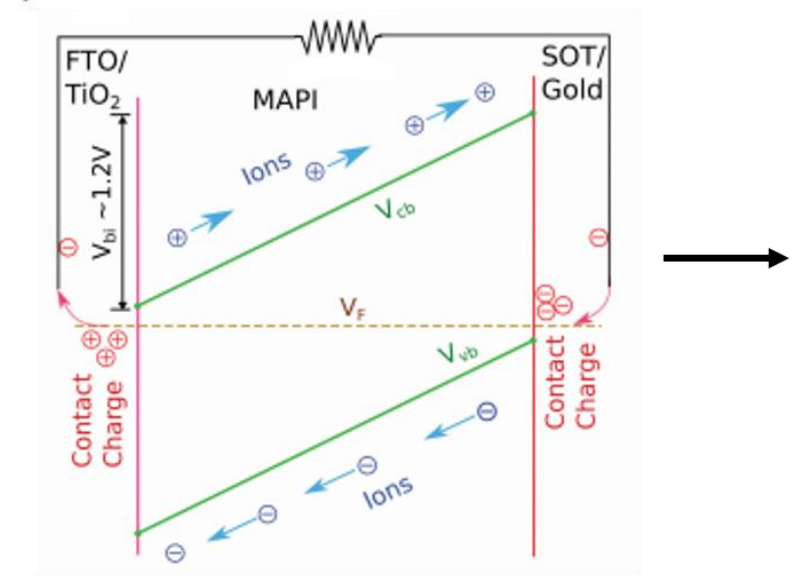

(b)

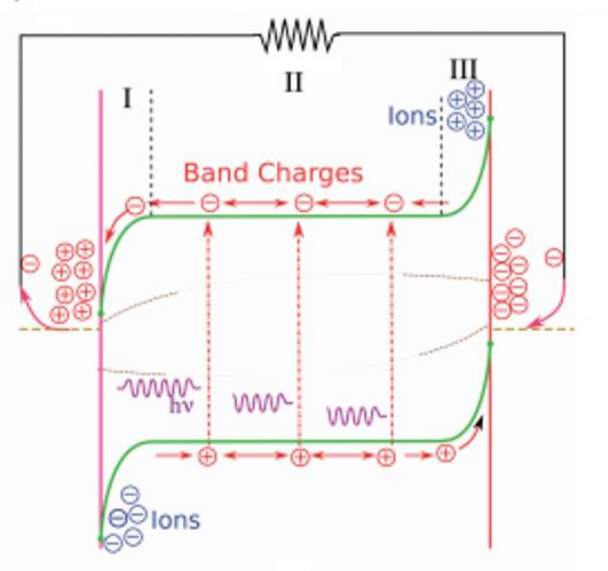

(c)

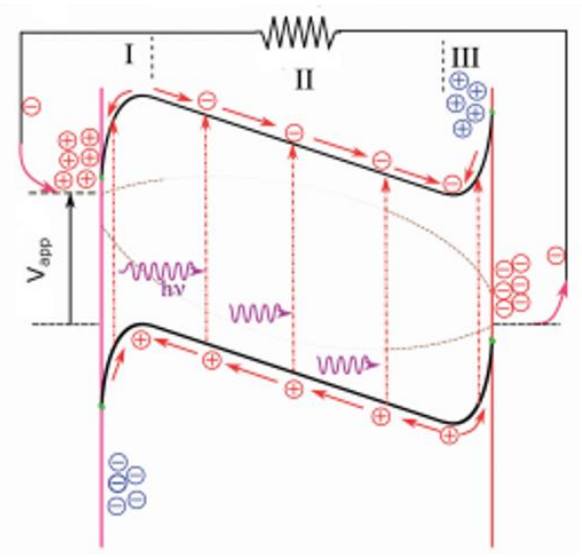

Figure 3.5 (a) n-i-p model of the perovskite solar cell, with a built-in potential $V_{b i}$ through the absorber that is the difference in workfunctions of the selective contacts. At equilibrium, the ions drift towards the respective contacts and shield the $V_{b i}$, creating a bulk field-free region with sharp potential drops at the contacts to extract photogenerated charges as shown in (b). (c) shows the effect of applied forward bias that tilts the bands unfavourably for carrier extraction. Regions I, III contain sharp potential drops due to the ions and region II is the bulk. Adapted from Ref. 6, licensed by CC BY 3.0, https://creativecommons.org/licenses/by/3.0/.

\subsection{Interfacial charge extraction and EQE}

After developing suitable models to account for the transport and accumulation of charge and its subsequent recombination at the ESL/perovskite interface, the next step in understanding the PSC operation was to identify the nature of charge extraction at the interfaces, where there are strong potential drops modulated by the ions. It must be mentioned that charge extraction is assumed to be ideal in the SPM and hence, a more detailed model would require knowledge of this effect. Therefore, IMPS measurements were carried out at SC conditions on MAPI solar cells. IMPS involves the application of a small perturbation of input photon flux $\tilde{\phi}$ and subsequent measurement of the modulated 
current response $\tilde{\jmath}$, scanned over a range of frequencies. Therefore, the IMPS transfer function is the frequency dependent $\mathrm{EQE}$ of the solar cell, given by

$$
Q(\omega)=\frac{1}{q} \frac{\tilde{J}}{\widetilde{\phi}}=\frac{\tilde{J}}{\widetilde{J \phi}}
$$

and the LF limit is then given by

$$
Q(0)=\frac{d \bar{\jmath}}{d \overline{J_{\phi}}}
$$

It is important to note that in a standard EQE measurement, it is the quantity in Equation 53 that is measured, though at a non-zero frequency, with an input modulated photon flux at a given wavelength, followed by measurement of the modulated current response, ultimately scanning over the entire range of wavelengths of interest. The modulation is provided internally by the measurement device or is provided either mechanically using a chopper or from directly modulating the light source. This experiment is generally carried out under a DC white light bias. Therefore, a proper definition of the EQE must be provided, depending on the nature of the measurement. This can be made by starting with a general relation between the DC extracted photocurrent $\overline{J_{e}}$ and the DC input photon flux expressed as a current density $\overline{J_{\phi}}$, given by

$$
\overline{J_{e}}(\lambda)=k{\overline{J_{\phi}}}^{n}(\lambda)
$$

where $k$ is a proportionality constant and $n$ is an exponent. Therefore, the steady state EQE can be defined as

$$
E Q E_{P V-s s}=\frac{\overline{J_{e}}}{\overline{J_{\phi}}}=k{\overline{J_{\phi}}}^{n-1}
$$

The EQE obtained from small perturbation methods or differential EQE can be defined as

$$
E Q E_{P V-\text { diff }}=\frac{d \overline{J_{e}}}{d \overline{J_{\phi}}}=\mathrm{n} k \bar{J}_{\phi}^{n-1}
$$

From Equations 55 and 56, it can be seen that $E Q E_{P V-s S}=E Q E_{P V-\text { diff }}$ only when $n=1$ ie: when the photocurrent response is linear with respect to the input photon flux. Therefore, care must be taken in defining the nature of the EQE that is being measured and reported. After establishing definitions for the EQE of a solar cell, IMPS measurements were made on $\approx 15 \%$ efficient, regular MAPI solar cells. All IMPS measurements were made with blue $(470 \mathrm{~nm})$ light to avoid absorption losses, ensuring that the EQE values obtained reflect the internal quantum efficiency (IQE) related to transport and recombination of carriers only. The obtained IMPS Q-plane (Q',-Q') plot is shown in Figure 3.6(c), with an arc in the upper quadrant at high frequencies followed by an arc in the lower quadrant at low frequencies. A small arc in the upper quadrant is also observed at intermediate frequencies, which is discussed subsequently. The evolution of the real part of the IMPS transfer function versus frequency is shown in Figure 3.6(d), indicating that the EQE of the PSC can vary strongly depending on the frequency at which it is measured. This was confirmed experimentally from standard EQE measurements by varying the chopper frequency. As can be seen in Figure 3.6(a) and (b), the variation in 
the EQE is as high as $10 \%$ for chopper frequencies between $10-500 \mathrm{~Hz}$ with or without a DC bias light intensity. This explains the large variation in the $j_{s c}$ values reported in literature, obtained from integration of the EQE over the solar spectrum. This indicates that rigid protocols are required to report the EQE of PSCs at standard chopper frequencies in a typical differential spectral response measurement, preferentially by measuring the response with several different frequencies and/or reporting the perturbation frequency used for the measurement.
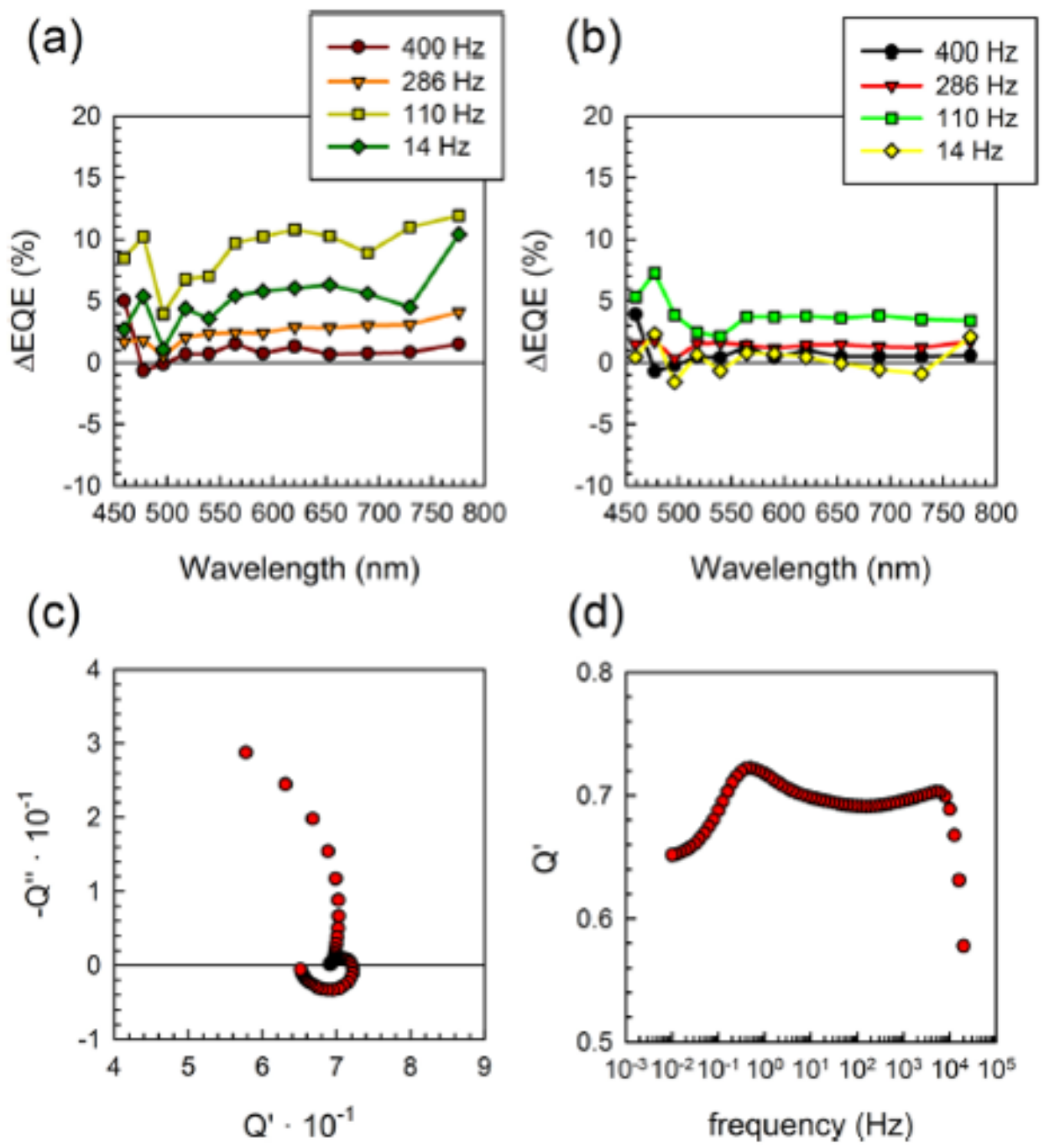

Figure 3.6 (a, b) Change in external quantum efficiency (EQE) for different chopper frequencies as shown in legend in dark and $10 \mathrm{~mW} / \mathrm{cm}^{2} \mathrm{DC}$ white light illumination respectively, with $500 \mathrm{~Hz}$ value as reference. (c) IMPS Q-plane plot measured under 33 $\mathrm{mA} / \mathrm{cm}^{2}$ blue $(470 \mathrm{~nm})$ light DC bias and (d) variation of real part of the IMPS transfer function with frequency as obtained from data of (c).

The evolution of the EQE of the PSC shows a unique reduction at low frequencies, below $10 \mathrm{~Hz}$, as seen from Figure 3.6(d). In order to understand this phenomenon, the basic solar cell model is shown in Figure 3.7(a), consisting of a parallel combination of 
chemical capacitance $C_{\mu}$ and recombination resistance $R_{r e c}$, with a current source in parallel to these elements that is related to photogeneration of carriers. The photocurrent is driven through the series resistance $R_{S}$. The IMPS response of this model can be easily explained by considering that the total current driven through the series resistance depends on the resistance of the $R_{\text {rec }} \| C_{\mu}$ line. At high frequencies, the capacitor acts like a short circuit and hence, there is no current extracted and at low frequencies, the capacitor acts as an open circuit, yielding a net resistance for the line governed by $R_{r e c}$. Therefore, the IMPS Q-plane response (Figure 3.7(b)) is an arc in the first quadrant with a characteristic frequency given by $\omega_{H F}=\left(R_{S} C_{\mu}\right)^{-1}$ and the HF and LF intercepts on the Q'-axis are 0 and $\left[1+\left(R_{S} / R_{r e c}\right)\right]^{-1}$ respectively. Therefore, the response can be considered similar to an $\mathrm{RC}$ attenuator where the capacitor controls the frequency-dependent response through the equipotential lines of the circuit.

(a)

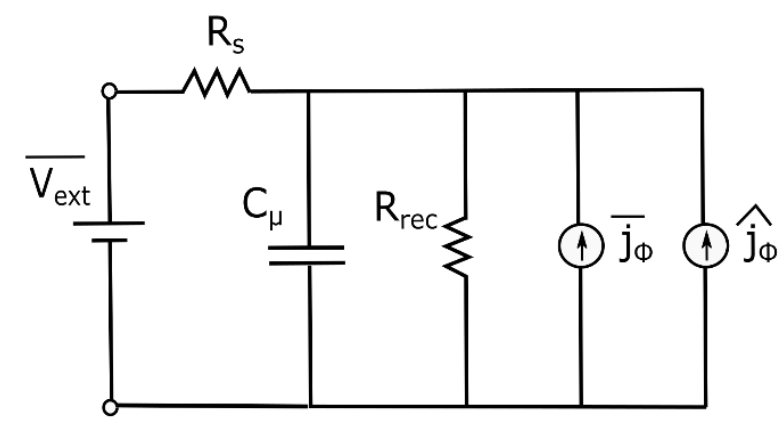

(c)

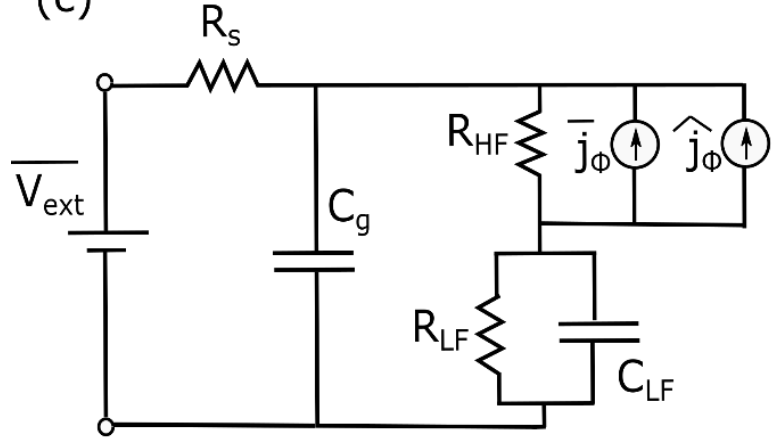

(b)

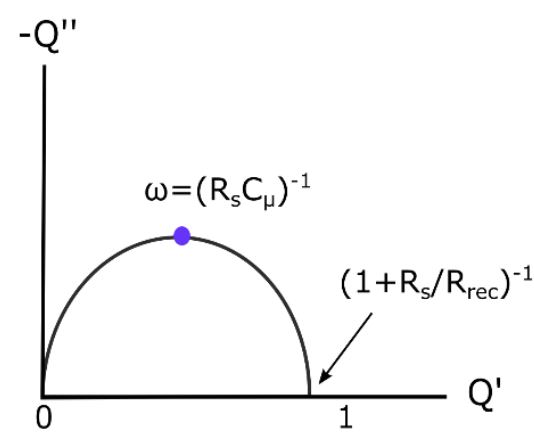

(d)

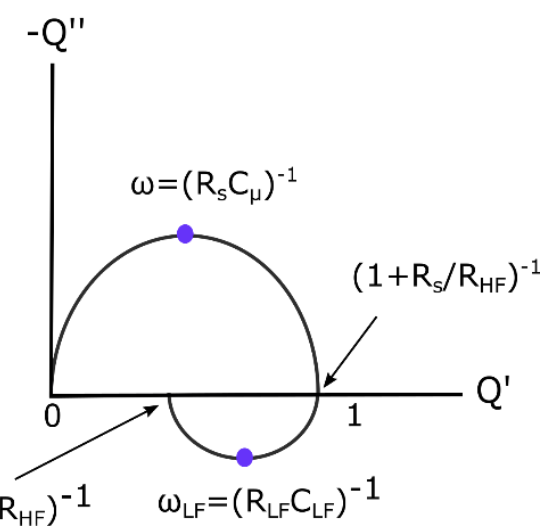

Figure 3.7 (a) Basic solar cell model and (c) perovskite solar cell model with corresponding IMPS Q-plane response ((b) and (d) respectively). $R_{S}, R_{r e c}, R_{H F}$ and $R_{L F}$ are the series resistance, recombination resistance, high frequency and low frequency resistances respectively. $C_{\mu}, C_{g}$ and $C_{L F}$ are the chemical, geometric and low frequency capacitances respectively. $\overline{J_{\phi}}$ and $\widehat{J_{\phi}}$ are the DC and modulated input photon current densities respectively. 
However, in the case of a PSC, the current source is placed only across the HF resistance, which means the potential drops encountered by the input and output current densities are not the same. This shows that the total photovoltage is separated in the PSC internally, as seen in Figure 3.7(c), which could be related to the fact that the LF phenomena are related to ionic accumulation at the interfaces, since the ionic densities are unaffected under illumination. The IMPS transfer function of the perovskite solar cell model shown in Figure 3.7(c) is given by

$$
Q(\omega)=\left(1+\frac{R_{S}}{R_{H F}}+i \omega R_{S} C_{g}+\left[\frac{R_{L F}}{R_{H F}}\right]\left[\frac{1+i \omega R_{S} C_{g}}{1+i \omega R_{L F} C_{L F}}\right]\right)^{-1}
$$

The HF arc in this case is again created by the time constant formed by $R_{S}$ and $C_{g}$, as described in Section 2.2. $C_{g}$ is the bulk dielectric or geometric capacitance of the perovskite absorber that is related to net polarisation of the sample over the device thickness, akin to a parallel-plate capacitor. This capacitance has also been well established from IS measurements for the PSC, described in Section 1.2.3.3.1. At low frequencies, the LF capacitor becomes a blocking element, which causes the LF resistor to act like an extra series resistor for the extracted photocurrent. This reduces the measured EQE, forming an arc in the lower quadrant (Figure 3.7(d)) depending on the time constant formed between $R_{L F}$ and $C_{L F}$. The Q-plane response, EC, time constants and intercepts for both the basic solar cell model and the PSC are summarised in Figure 3.7.

Therefore, the slow kinetic effects observed in recombination times and voltage decays are inevitably reflected in the charge extraction kinetics of the PSC. The physical mechanism governing this reduction in the EQE, which is quantitatively described by IMPS measurements, is governed strongly by the interactions between the ions and electronic carriers at the perovskite/selective contact interfaces, whose further understanding is critical to resolve this effect. This work provided a novel interpretation of the IMPS response of PSCs and the meaning of the arcs, their intercepts and the time constants observed in a Q-plane plot. 


\subsection{Developing the equivalent circuit of the PSC}

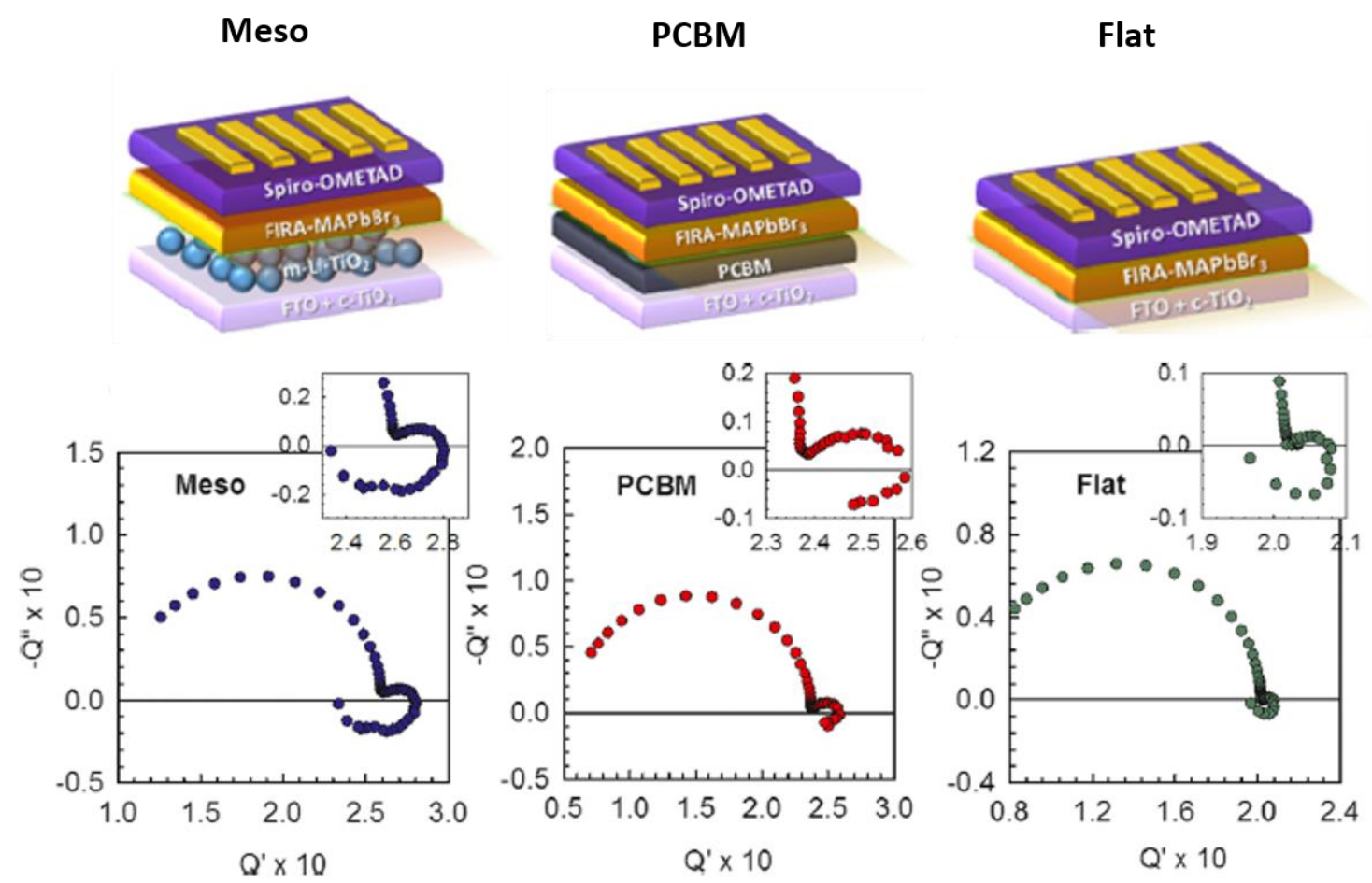

Figure 3.8 Schematic of device configurations of the different PSCs measured and corresponding IMPS Q-plane plots at OC conditions using blue (470 nm) light. Insets show the zoomed image of the intermediate and low frequency response.

After establishing the PSC EC at SC conditions, the next step was to confirm this EC at $\mathrm{OC}$ conditions, considering that the EC must remain the same in all conditions. In addition, the IS response of PSCs is known to be quite variable in general, depending a lot on the nature of the samples. This makes it difficult to obtain a reproducible and repeatable response and hence model from IS measurements. The application of a DC biasing voltage for extended periods of time in these measurements also causes degradation in the performance. ${ }^{14}$ Therefore, it becomes important to validate and complement the results from IS measurements on PSCs with another small perturbation technique. Hence, IMPS measurements on PSCs at OC conditions were studied systematically for the first time. The measured PSCs were stable and reproducible $\mathrm{CH}_{3} \mathrm{NH}_{3} \mathrm{PbBr}_{3}$ cells with different ESLs, whose configuration and $j-V$ curves are shown in Figure 3.8 and 3.10(a) respectively. It can be seen that the ESL plays a strong role in the obtained $j_{s c}$ value and the FF.

The IMPS Q-plane response of these cells at OC conditions are shown in Figure 3.8. The reduced values of the real part of the transfer function at $\mathrm{OC}$ conditions that represents the EQE of the solar cell compared to SC conditions can be understood from the fact that the intercept values are governed by the recombination resistance $R_{\text {rec }}$, as seen from Figure 3.7. All the cells show a response similar to that of Figure 3.7(d), with a HF arc in 
the upper quadrant and an LF arc in the lower quadrant. However, upon closer inspection, a small intermediate frequency (IF) arc can be observed in the upper quadrant at characteristic frequencies around $10 \mathrm{~Hz}$, as seen in the inset of Figure 3.8. Therefore, the EC of the PSC was further extended to include an IF capacitor charged through a resistor $R_{I F}$, as seen in Figure 3.9(a). The simulated Q-plane response of the EC reproduces the three arcs observed, while the simulated IS response yields the two arcs in the upper quadrant this is usually observed for stable and efficient PSCs. The corresponding time constants for the arcs observed in both IMPS and IS measurements at OC conditions are shown in Figure 3.9(b) and 3.9(c) respectively. The inverse of these time constants correspond to the characteristic frequencies of each process, represented hereafter as $\omega_{\tau}$, where $\tau$ is the corresponding time constant represented as the product of a resistance and capacitance. Fitting of the Q-plane data of the cells yielded values for the capacitances and resistances, which are summarised in Table 3.1.

\begin{tabular}{|l|l|l|l|l|l|l|l|l|}
\hline & $\begin{array}{l}R_{s} \\
\left(\Omega \cdot \mathrm{cm}^{2}\right)\end{array}$ & $\begin{array}{l}R_{H F} \\
\left(\Omega \cdot \mathrm{cm}^{2}\right)\end{array}$ & $\begin{array}{l}R_{L F} \\
\left(\Omega \cdot \mathrm{cm}^{2}\right)\end{array}$ & $\begin{array}{l}R_{I F} \\
\left(\Omega \cdot \mathrm{cm}^{2}\right)\end{array}$ & $\begin{array}{l}C_{g, I M P S} \\
\left(\mathrm{~F} \cdot \mathrm{cm}^{-2}\right)\end{array}$ & $\begin{array}{l}C_{g, I S} \\
\left(\mathrm{~F} \cdot \mathrm{cm}^{-2}\right)\end{array}$ & $\begin{array}{l}C_{I F} \\
\left(\mathrm{~F} \cdot \mathrm{cm}^{-2}\right)\end{array}$ & $\begin{array}{l}C_{L F} \\
\left(\mathrm{~F} \cdot \mathrm{cm}^{-2}\right)\end{array}$ \\
\hline Meso & 95 & 37 & 26 & 366 & $2.6 \times 10^{-7}$ & $2 \times 10^{-7}$ & $1.3 \times 10^{-4}$ & $4.8 \times 10^{-2}$ \\
\hline Flat & 125 & 33 & 9 & 994 & $2.0 \times 10^{-7}$ & $4 \times 10^{-7}$ & $8.0 \times 10^{-4}$ & $7.0 \times 10^{-2}$ \\
\hline PCBM & 125 & 43 & 11 & 392 & $2.5 \times 10^{-7}$ & $3 \times 10^{-7}$ & $8.0 \times 10^{-4}$ & $1.8 \times 10^{-1}$ \\
\hline
\end{tabular}

Table 3.1 Obtained parameters from fitting of IMPS data at OC conditions. $C_{g, I S}$ is the geometric capacitance value extracted from IS measurements at OC conditions.

Firstly, it can be noticed that the bulk dielectric capacitance $C_{g}$ obtained from IMPS is in good agreement with that obtained from IS measurements (see also Figure 1.13(c)). The large LF capacitance of the order of a few $\mathrm{mF}$, attributed to the accumulation of holes and cations at the ESL/perovskite interface, and the values of $R_{H F}$ and $R_{L F}$ are also well established from IS measurements. ${ }^{15}$ The IF capacitance observed only from IMPS measurements is two orders smaller than the LF capacitance. This was attributed to the accumulation of anions at the perovskite/HSL interface that likely includes an electronic component, due to the large values of capacitance observed. Furthermore, the IF resistance varies significantly for the three cells. The significance of the IF resistance is apparently unclear because it is in series with the IF capacitor and hence, cannot contribute to the steady state or DC response of the PSC. However, it must be noted that the $j-V$ curve of a solar cell is actually measured at a certain voltage scan rate, however slow it may be, which means the IF resistance can contribute to the device resistance depending on its IS time constant and the $j-V$ scan frequency. The net impedance of the EC shown in Figure 3.9(a) is given by

$$
Z_{n e t}(\omega)=R_{s}+\left(i \omega C_{g}+\frac{1}{R_{I F}+\frac{1}{i \omega C_{I F}}}+\frac{1}{R_{H F}+\frac{1}{\frac{1}{R_{L F}}+i \omega C_{L F}}}\right)^{-1}
$$

For $\omega_{\tau_{H F}} \gg \omega \gg \omega_{\tau_{I F}}, \omega_{\tau_{L F}}$ in the Z-plane plot of Figure 3.9(c), we have,

$$
Z_{\text {net }}=R_{S}+\left(\frac{1}{R_{H F}}+\frac{1}{R_{I F}}\right)^{-1}
$$


Therefore, the net device resistance at these frequencies is controlled by the parallel combination of the HF recombination resistance and the IF charging resistance. At OC conditions, the HF recombination resistance is very small, of the order of a few tens of $\Omega \cdot \mathrm{cm}^{2}$, and hence dominates the net impedance. However, at lower forward biases, the recombination resistance values increase exponentially, consequently making the IF resistance control the net device resistance. This is shown in the simulations of Figure 3.10(b), where the frequency range of standard $j-V$ scans coincides with this situation. Therefore, the IF resistance is a critical factor in controlling the net device resistance of PSCs, which can explain the large disparity in FFs for different PSCs, especially the large reduction in $\mathrm{FF}$ when scanning from $\mathrm{R}$ to $\mathrm{F}$ voltages. ${ }^{4}$
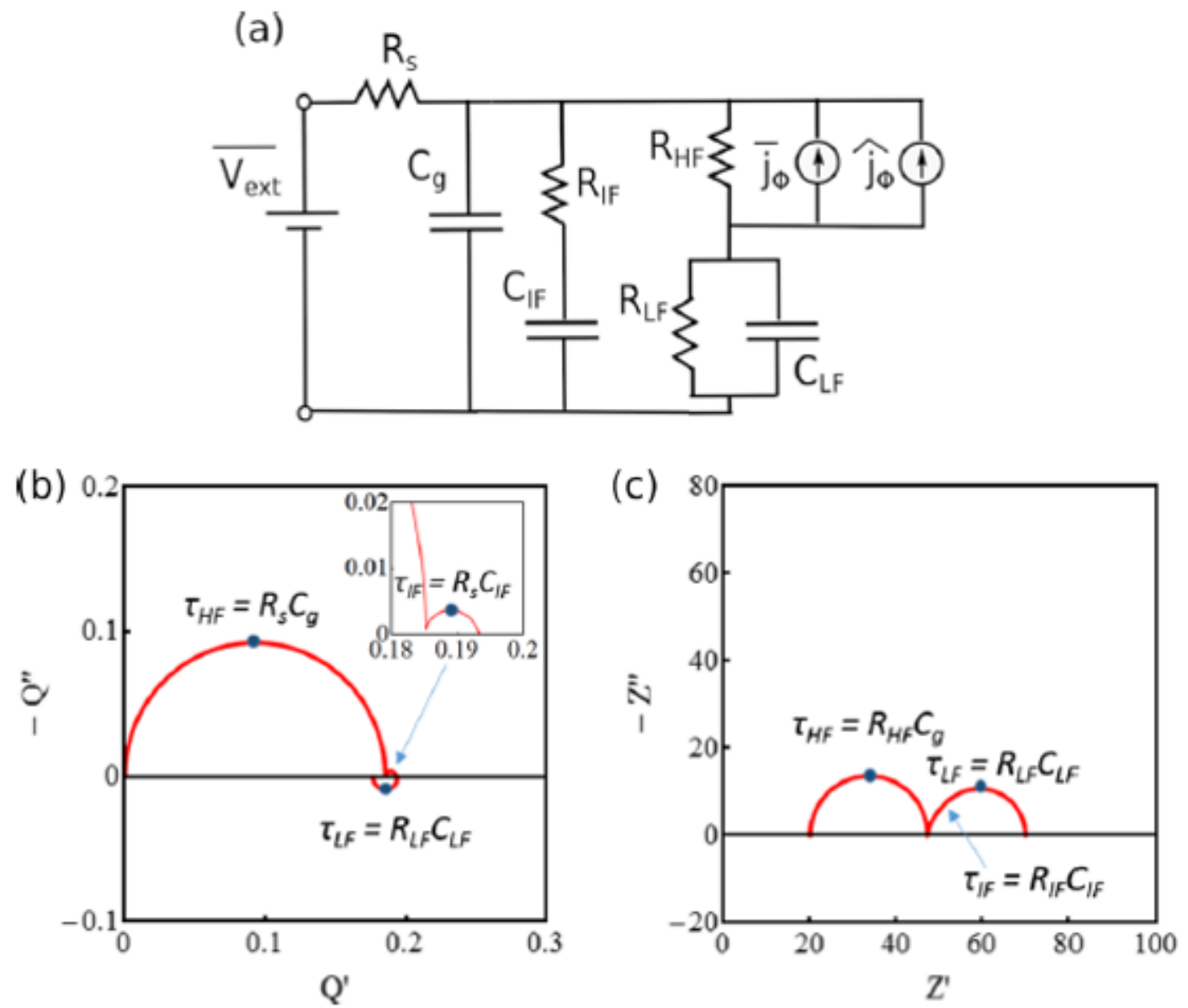

Figure 3.9 (a) Proposed EC for the PSC. (b) Simulated IMPS $Q$-plane plot and (c) IS Zplane plot at OC conditions using circuit of (a), with corresponding time constants at OC shown. Parameters used were $R_{s}=120$ and $20 \Omega \cdot \mathrm{cm}^{2}$ for (b) and (c) respectively, $R_{H F}=$ $30 \Omega \cdot \mathrm{cm}^{2}, R_{L F}=20 \Omega \cdot \mathrm{cm}^{2}, R_{I F}=300 \Omega \cdot \mathrm{cm}^{2}, C_{g}=10^{-7} \mathrm{~F} \cdot \mathrm{cm}^{-2}, C_{I F}=10^{-4} \mathrm{~F} \cdot \mathrm{cm}^{-2}, C_{L F}$ $=10^{-2} \mathrm{~F} \cdot \mathrm{cm}^{-2}$.

It must also be noted that the values of $R_{S}$ obtained from IMPS are of the order of 100 $\Omega \cdot \mathrm{cm}^{2}$, while IS measurements yield values of around $20 \Omega \cdot \mathrm{cm}^{2}$. This points to the fact that the EC of Figure 3.9(a) is incomplete, and likely includes an extra resistance in series 
with $R_{S}$ or in parallel along the other lines of the EC. An obvious resistance that needs to be considered is the transport resistance $R_{t}\left(\Omega \cdot \mathrm{cm}^{2}\right)$ given by

$$
R_{t}=\frac{L}{\sigma}
$$

where $\sigma=q p \mu_{p}$ is the conductivity of holes, $L$ is the thickness of the sample, $p$ is the density of majority carrier holes and $\mu_{p}$ is the hole mobility. Taking an average value of $40 \mathrm{~cm}^{2} \mathrm{~V}^{-1} \mathrm{~s}^{-1}$ for $\mu_{p}$ from reported values ${ }^{16}$ in the literature and a hole density of $2 \times 10^{16}$ $\mathrm{cm}^{-3}$ corresponding to $90 \mathrm{~mW} \cdot \mathrm{cm}^{2} \mathrm{DC}$ illumination of blue $(470 \mathrm{~nm})$ photons impinging on the sample yields a $R_{t}$ value of $\sim 2.3 \times 10^{-4} \Omega \cdot \mathrm{cm}^{2}$ for a $300 \mathrm{~nm}$ thick sample. This value is negligibly small and hence, the transport resistance is not a contributing factor to the EC, indicating that the extra resistance should be placed along the parallel lines of the EC.

(a)

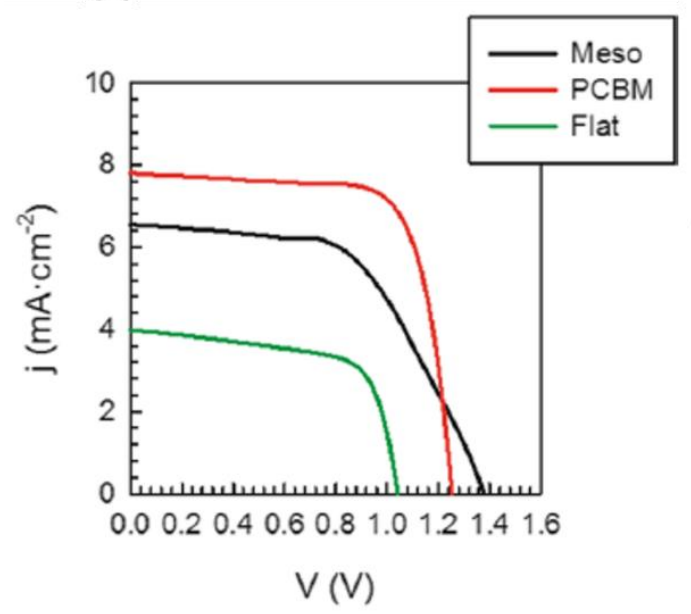

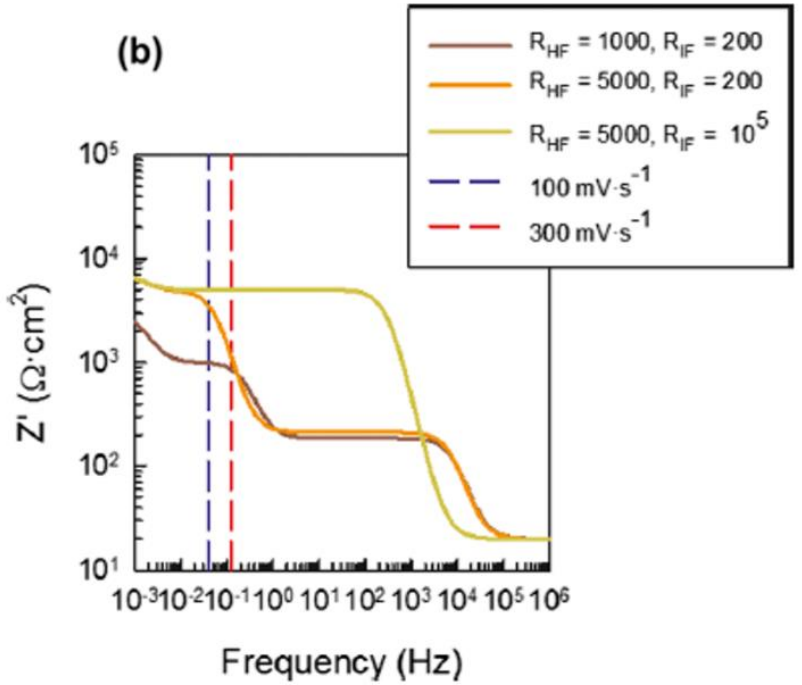

Figure 3.10 (a) $j-V$ curves of the different PSCs measured and (b) simulated variation of net device resistance depending on the values of the IF resistance in relation to the HF and LF resistances shown in the legend. The dotted lines indicate typical scan rates used in a $j-V$ measurement. The effect of the IF resistance is to alter the FF of the cells as seen in (a), with Meso cells showing the lowest FF and IF resistance (see Table 3.1).

The obtained results and insights from the IMPS measurements on PSCs are very significant. The observation of an IF process in IMPS that is not observable in IS indicates that the time constants formed in either measurement are different, as is shown in Figure 3.9. In IS, the IF time constant is similar in value to the LF time constant, which prevents them being decoupled, unlike in IMPS where the arcs in the upper quadrant are always formed by the series resistance and capacitors in associated lines of the circuit and hence, the IF and LF time constants are different and decoupled. Furthermore, these time constants do not have any physical meaning unless derived from a specific transport/recombination model, which currently does not exist for the PSC. Therefore, associating electronic and ionic transport phenomena to the time constants observed in an 
IMPS Q-plane plot, which is a frequently adopted procedure in the field, is erroneous. While observation of ionic transport cannot be ruled out due to their slow drift, the electronic transport is very difficult to observe, with the characteristic frequency of diffusion $\omega_{d}$ given by ${ }^{17}$

$$
\omega_{d}=\frac{D}{L^{2}}
$$

where $D$ is the diffusion coefficient of the electronic carrier and $L$ is the device thickness. Taking a $D$ value of $1 \mathrm{~cm}^{2} \mathrm{~s}^{-1}$ from reported values in the literature and a device thickness of $300 \mathrm{~nm}$ yields a $\omega_{d}$ value of $1.1 \times 10^{9} \mathrm{~Hz}$, which is well out of the frequency range of small perturbation techniques such as IS and IMPS. In addition, the characteristic response of transport in both $\mathrm{IS}^{17}$ and IMPS ${ }^{18}$ is the observation of a $45^{\circ}$ straight line at high frequencies, which is not observed for PSCs. Hence, in the absence of an established transport/recombination model to study the small perturbation response of PSCs, it is imperative to establish the underlying EC from different small perturbation techniques to develop a suitable transport/recombination model for the solar cell that is being probed.

In summary, the time constants observed in IMPS spectra are not related to transport of photogenerated carriers in the perovskite absorber or in the selective contacts, as is commonly interpreted. Secondly, it allows the identification of an extra capacitance from IMPS that is not observable from IS measurements due to different combinations of the time constants involved in each measurement. This capacitance is one or two orders smaller than the LF capacitance and the resistance associated to charging this capacitor is a prominent factor in determining the net resistance of the PSC at low voltages, where the recombination resistances are very large in magnitude. This effect can explain the large variations in FF for PSCs, whose origins and mechanisms are still unresolved. The resulting EC is able to reproduce both the IMPS and IS response of PSCs effectively, providing a reliable and robust focal point for understanding the physical mechanisms of transport, charge storage and recombination in a device that shows a huge variation in properties and response depending on materials and methods.

\subsection{Conclusions}

The following conclusions are drawn about the working mechanisms of PSCs from the work carried out in this thesis:

- There is no correlation observed between the contact workfunctions and the maximum $V_{O c}$ obtained. This means that the built-in voltage in PSCs is mostly absorbed at the perovskite/selective contact interfaces in a small layer, with the bulk being electrical-field free and hence, the predominant mode of transport of photocarriers is diffusion. Therefore, it is recombination in the bulk and at interfaces that control the maximum $V_{o c}$, rather than charge collection due to an electrical field.

- The slow release of charges accumulated at the interfaces control the kinetics of several parameters of the PSC such as the recombination rates and the $V_{o c}$ 
and is inevitably reflected in the EQE of PSCs, showing a singular evolution at low frequencies that is related to internal displacement of the photovoltage in the PSC.

- Transport of photogenerated carriers within the absorber is excellent in high efficiency PSCs, evidenced by the absence of characteristic transport signals in both IMPS and IS.

- IMPS measurements show the existence of an additional capacitor of the order of $10^{-4} \mathrm{~F} \cdot \mathrm{cm}^{-2}$, related to charging of the perovskite/HSL interface, that is not observable from IS measurements due to the nature of the time constants formed in either measurement. The resistance associated to charging this capacitor is crucial in controlling the net device resistance at low forward biases and hence the fill factor of the device.

- The hysteretic trends and performance metrics of high efficiency PSCs are predominantly based on variations in $\mathrm{FF}$ and/or $V_{o c}$, strongly controlled by ionic and electronic storage and subsequent recombination at the perovskite/selective contact interfaces. Therefore, these interfaces must be tuned efficiently to gain control over the capacitive properties and their interactions with the ions that influence the kinetic behaviour of PSCs. 


\subsection{References}

1. Berhe, T. A.; Su, W.-N.; Chen, C.-H.; Pan, C.-J.; Cheng, J.-H.; Chen, H.-M.; Tsai, M.-C.; Chen, L.-Y.; Dubale, A. A.; Hwang, B.-J. Organometal halide perovskite solar cells: degradation and stability. Energy \& Environmental Science 2016, 9 (2), 323-356.

2. Domanski, K.; Correa-Baena, J.-P.; Mine, N.; Nazeeruddin, M. K.; Abate, A.; Saliba, M.; Tress, W.; Hagfeldt, A.; Grätzel, M. Not all that glitters is gold: metalmigration-induced degradation in perovskite solar cells. ACS Nano 2016, 10 (6), 63066314.

3. Azpiroz, J. M.; Mosconi, E.; Bisquert, J.; De Angelis, F. Defect migration in methylammonium lead iodide and its role in perovskite solar cell operation. Energy \& Environmental Science 2015, 8 (7), 2118-2127.

4. Tress, W.; Marinova, N.; Moehl, T.; Zakeeruddin, S. M.; Nazeeruddin, M. K.; Gratzel, M. Understanding the rate-dependent J-V hysteresis, slow time component, and aging in $\mathrm{CH}_{3} \mathrm{NH}_{3} \mathrm{PbI}_{3}$ perovskite solar cells: the role of a compensated electric field. Energy \& Environmental Science 2015, 8 (3), 995-1004.

5. Unger, E. L.; Hoke, E. T.; Bailie, C. D.; Nguyen, W. H.; Bowring, A. R.; Heumuller, T.; Christoforo, M. G.; McGehee, M. D. Hysteresis and transient behavior in current-voltage measurements of hybrid-perovskite absorber solar cells. Energy \& Environmental Science 2014, 7 (11), 3690-3698.

6. Belisle, R. A.; Nguyen, W. H.; Bowring, A. R.; Calado, P.; Li, X.; Irvine, S. J. C.; McGehee, M. D.; Barnes, P. R. F.; O'Regan, B. C. Interpretation of inverted photocurrent transients in organic lead halide perovskite solar cells: proof of the field screening by mobile ions and determination of the space charge layer widths. Energy \& Environmental Science 2017, 10 (1), 192-204.

7. Lopez-Varo, P.; Jiménez-Tejada, J. A.; García-Rosell, M.; Anta, J. A.; Ravishankar, S.; Bou, A. n.; Bisquert, J. Effects of Ion Distributions on Charge Collection in Perovskite Solar Cells. ACS Energy Letters 2017, 2, 1450-1453.

8. Zarazua, I.; Bisquert, J.; Garcia-Belmonte, G. Light-induced space-charge accumulation zone as photovoltaic mechanism in perovskite solar cells. The Journal of Physical Chemistry Letters 2016, 7 (3), 525-528.

9. Gottesman, R.; Lopez-Varo, P.; Gouda, L.; Jimenez-Tejada, J. A.; Hu, J.; Tirosh, S.; Zaban, A.; Bisquert, J. Dynamic Phenomena at Perovskite/Electron-Selective Contact Interface as Interpreted from Photovoltage Decays. Chem 2016, 1 (5), 776-789.

10. Juarez-Perez, E. J.; Sanchez, R. S.; Badia, L.; Garcia-Belmonte, G.; Kang, Y. S.; Mora-Sero, I.; Bisquert, J. Photoinduced giant dielectric constant in lead halide perovskite solar cells. The Journal of Physical Chemistry Letters 2014, 5 (13), 2390-2394. 
11. Stranks, S. D.; Eperon, G. E.; Grancini, G.; Menelaou, C.; Alcocer, M. J.; Leijtens, T.; Herz, L. M.; Petrozza, A.; Snaith, H. J. Electron-hole diffusion lengths exceeding 1 micrometer in an organometal trihalide perovskite absorber. Science 2013, 342 (6156), 341-344.

12. Shi, D.; Adinolfi, V.; Comin, R.; Yuan, M.; Alarousu, E.; Buin, A.; Chen, Y.; Hoogland, S.; Rothenberger, A.; Katsiev, K. Low trap-state density and long carrier diffusion in organolead trihalide perovskite single crystals. Science 2015, 347 (6221), 519-522.

13. Bergmann, V. W.; Guo, Y.; Tanaka, H.; Hermes, I. M.; Li, D.; Klasen, A.; Bretschneider, S. A.; Nakamura, E.; Berger, R. d.; Weber, S. A. Local time-dependent charging in a perovskite solar cell. ACS Applied Materials \& Interfaces 2016, 8 (30), 19402-19409.

14. Pitarch-Tena, D.; Ngo, T. T.; Vallés-Pelarda, M.; Pauporté, T.; Mora-Seró, I. Impedance Spectroscopy Measurements in Perovskite Solar Cells: Device Stability and Noise Reduction. ACS Energy Letters 2018, 3 (4), 1044-1048.

15. Zarazua, I.; Han, G.; Boix, P. P.; Mhaisalkar, S.; Fabregat-Santiago, F.; MoraSeró, I.; Bisquert, J.; Garcia-Belmonte, G. Surface Recombination and Collection Efficiency in Perovskite Solar Cells from Impedance Analysis. The Journal of Physical Chemistry Letters 2016, 7 (24), 5105-5113.

16. Herz, L. M. Charge-carrier mobilities in metal halide perovskites: fundamental mechanisms and limits. ACS Energy Letters 2017, 2 (7), 1539-1548.

17. Bisquert, J. Theory of the impedance of electron diffusion and recombination in a thin layer. The Journal of Physical Chemistry B 2002, 106 (2), 325-333.

18. Dloczik, L.; Ileperuma, O.; Lauermann, I.; Peter, L. M.; Ponomarev, E. A.; Redmond, G.; Shaw, N. J.; Uhlendorf, I. Dynamic Response of Dye-Sensitized Nanocrystalline Solar Cells: Characterization by Intensity-Modulated Photocurrent Spectroscopy. The Journal of Physical Chemistry B 1997, 101 (49), 10281-10289. 


\section{Chapter 4}

\section{Publication 1}

\section{Surface Polarization Model for the Dynamic Hysteresis in Perovskite Solar Cells}

Ravishankar, S.; Almora, O.; Echeverría-Arrondo, C.; Ghahremanirad, E.; Aranda, C.; Guerrero, A.; Fabregat-Santiago, F.; Zaban, A.; Garcia-Belmonte, G.; Bisquert, J. The Journal of Physical Chemistry Letters 2017, 8 (5), 915-921. Reprinted with permission, Copyright 2017, American Chemical Society.

\section{Candidate's contribution}

\begin{tabular}{|c|c|}
\hline Nature of Contribution & Extent of Contribution \\
\hline $\begin{array}{l}\text { - Contributed to development of the } \\
\text { model } \\
\text { - Performed the simulations } \\
\text { - Performed fitting of experimental } \\
\text { data } \\
\text { - Wrote part of the manuscript } \\
\text { - Wrote first draft of the reply to the } \\
\text { referees }\end{array}$ & $60 \%$ \\
\hline
\end{tabular}

\section{Introduction}

This work establishes the role of the ions in creating an excess majority carrier density at the ESL/perovskite interface at forward bias, whose kinetics are controlled by the slow movement of the ions away from this interface. The capacitive discharge of these accumulated carriers and their recombination yield several of the unique hysteresis trends observed in PSCs and their dependence on scan rate, direction and pre-biasing.

\section{Published manuscript}




\title{
Surface Polarization Model for the Dynamic Hysteresis of Perovskite Solar Cells
}

\author{
Sandheep Ravishankar, ${ }^{1}$ Osbel Almora, ${ }^{1}$ Carlos Echeverría-Arrondo, ${ }^{1}$ Elnaz \\ Ghahremanirad, ${ }^{1,2}$ Clara Aranda, ${ }^{1}$ Antonio Guerrero, ${ }^{1}$ Francisco Fabregat- \\ Santiago, ${ }^{1}$ Arie Zaban, ${ }^{3}$ Germà Garcia-Belmonte, ${ }^{1}$ Juan Bisquert ${ }^{1 *}$
}

${ }^{1}$ Institute of Advanced Materials (INAM), Universitat Jaume I, 12006 Castelló, Spain

${ }^{2}$ Nano-photonics and Optoelectronics Research Laboratory (NORLab), Shahid Rajaee Teacher Training University, 16788- 15811, Lavizan, Tehran, Iran

${ }^{3}$ Department of Chemistry, Institute for Nanotechnology \& Advanced Materials, BarIlan University, Ramat Gan 52900, Israel.

Email: bisquert@uji.es

\begin{abstract}
The dynamic hysteresis of perovskite solar cells consists of the occurrence of significant deviations of the current density-voltage curve shapes depending on the specific conditions of measurement such as starting voltage, waiting time, scan rate and other factors. Dynamic hysteresis is a serious impediment to stabilized and reliable measurement and operation of the perovskite solar cells. In this paper, we formulate a model for the dynamic hysteresis based on the idea that the cell accumulates huge quantity of surface electronic charge at forward bias that is released on voltage sweeping, causing extra current over the normal response. The charge shows a retarded dynamics due to the slow relaxation of the accompanying ionic charge, causing variable shapes depending on scan rate or poling value and time. We show that the quantitative model provides a consistent description of experimental results and allows us to determine significant parameters of the perovskite solar cell both for the transient and steady state performance.
\end{abstract}

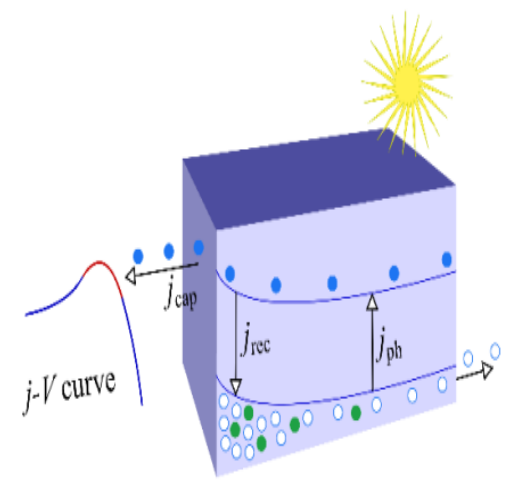


Lead halide perovskite has established a major scientific and technical research field in the search for new low cost versatile materials for solar energy conversion and optoelectronics. ${ }^{1-3}$ The current density-voltage $(j-V)$ performance characteristic of a solar cell is the central property that provides the main parameters of steady-state behaviour, namely the photovoltage $\left(V_{o c}\right)$, the short-circuit current $\left(j_{s c}\right)$ and the fill factor (FF), which when combined establish the power conversion efficiency at the specified solar cell conditions. Measuring the $j-V$ curve necessitates a dynamic measurement involving the sweeping of the voltage $V$ at least from open circuit to short circuit, or vice versa, while recording the current density $j$, which should provide a stable response representing the long term operation values of the solar cell under sunlight. Dynamic hysteresis of perovskite solar cells is the set of phenomena that make the current values fluctuate upon voltage cycling depending on details of measurement.

Since the advent of perovskite solar cells, dynamic hysteresis has been found to affect widely the supposedly steady state performance. ${ }^{4-6}$ The photocurrent displays diverse and sometimes apparently random behaviour as a function of the starting voltage, the scan velocity $b=d V / d t$ and the delay time $\Delta t$ between two consecutive voltage steps. ${ }^{6-7}$ This behaviour casts doubt on the validity of the performance values that are measured/reported, and it questions the efforts to improve the solar cell, requiring careful protocols to ensure a stabilized response. ${ }^{8-9}$ Dynamic hysteresis has been found in several types of solar cells in the past but it is especially challenging in the lead halide perovskite solar cell as it is a very large effect in typical slow measuring times and the phenomenology is widely varied. According to an extended opinion, the dynamic hysteresis indicates the existence of physical phenomena occurring in the perovskite solar cell that need to be understood. Some authors have reported hysteresis-free devices, ${ }^{10}$ but unfortunately, these are yet marginal in the field and do not represent most of the work that it is currently being carried out in the area. There is no shortage of explanations for the frequently observed hysteresis using a wide set of different hypotheses, mainly involving traps, ion motion, band bending, boundary layers, grain boundaries and other factors. Obviously, all these models capture to some extent the specific qualities of the hysteresis in current-voltage curves. What has not been achieved so far is a systematic and predictive model of how the different observed dynamic hysteresis effects depend on the previous conditioning and specific protocol of measurement. Furthermore, the model should have physical meaning consistent with observation obtained for different experimental techniques.

In this work, we will first describe the main trends of experimental observations reported so far, following which we will introduce a model based on the observation that the interface can be charged electronically that will quantitatively explain important characteristic features of phenomenology, consistently with important features previously observed by impedance spectroscopy and time transient methods. We will finish by showing experimental support that explains dynamic hysteresis in terms of large transient currents predicted by the surface polarization model and by calculating physical 
parameters by direct fitting of $j-V$ curves measured at different conditions.

\section{Characteristic features of dynamic hysteresis in perovskite solar cells}

We start the description of the features of dynamic hysteresis. In this paper, forward $(\mathrm{F})$ is the voltage around open circuit, and reverse $(\mathrm{R})$ is the voltage beyond short circuit. For example, we can sweep the voltage in the direction FR or in the other direction RF. But a FR scan will be very different if we cycle continuously in both directions, than if we wait a little bit with the cell sitting at constant $\mathrm{F}$ bias beyond $V_{o c}$ for a "preconditioning" or poling time. The value of the current will be characteristically larger and the efficiency is amplified. To check stability of response, one usually cycles RF and backwards FR and the result of such procedure is currently indispensable experimental evidence for reporting. But it turns out to be very different to make FR and RF in separate runs, than to cycle continuously the solar cell. The sensitivity of dynamic hysteresis to the details of measurements has thus been broadly acknowledged. The work of Unger et al. ${ }^{7}$ provided a sound recognition of these phenomena and the influence of the ionic conduction effects on the varying behaviours of the solar cells. Recent works have emphasized the role of contacts and ion migration effects in the occurrence of hysteresis and long time stability. ${ }^{11-12}$ 
(a)

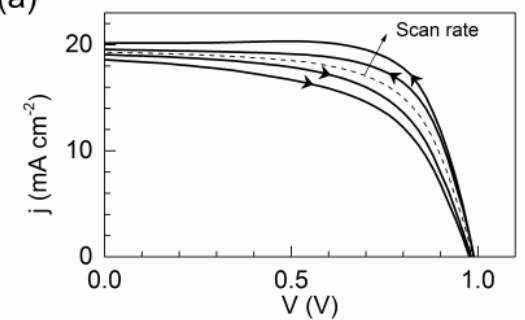

(c)

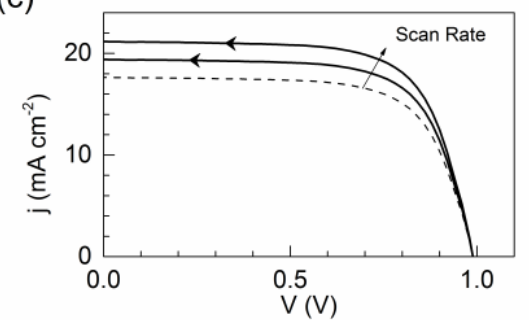

(e)

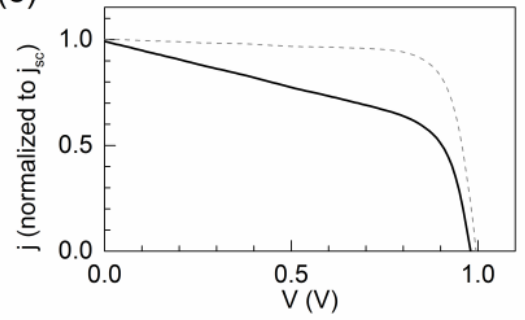

(g)

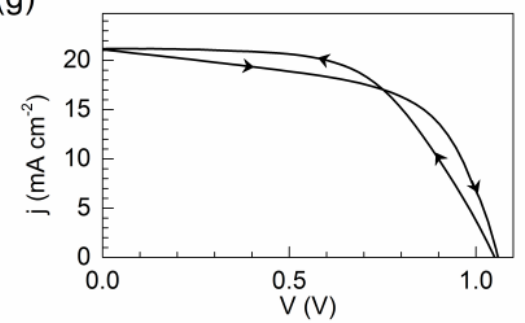

(b)

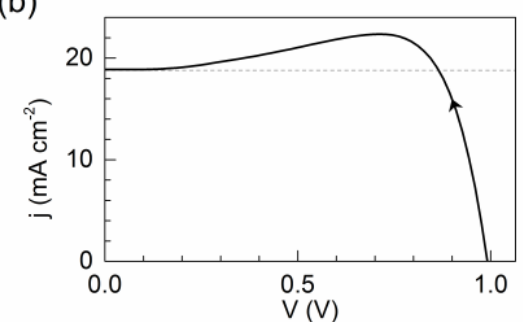

(d)

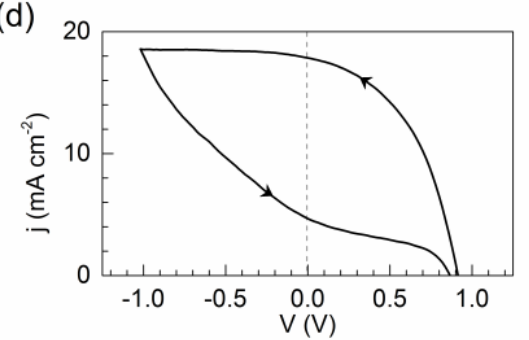

(f)

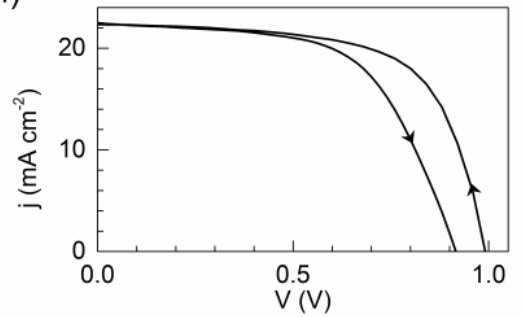

Fig. 1. Characteristic patterns of hysteresis behaviour in perovskite solar cells. The large arrows on the solid black lines indicate the scan sense. The black dashed line shows the steady state curve (a, c). In (b) and (d), the auxiliary dashed lines signal $j_{s c}$ and zero applied bias respectively, while in (e), it represents a typical diode curve.

In the very large number of published results, we can distinguish a specific set of characteristic patterns that need to be explained by models of dynamic hysteresis. These patterns are shown schematically in Fig. 1 and can be described as follows. Additional discussion, references and experimental measurements are presented in SI.

(a) Capacitive hysteresis. This behaviour can be readily understood as an instantaneous capacitive current added on the steady state diode curve. ${ }^{4,10,13-16}$ Here, it is apparent that the non-steady state curves with same voltage scan rate but opposite scan sense are symmetrically disposed with respect to the steady state current and additionally, the capacitive current increases linearly with scan rate.

(b) Starting from F to R, the current displays a maximum ("bump") and decreases to a 
stable photocurrent so that current close to $V_{o c}$ is actually higher than short-circuit current. This phenomenon has been widely observed with a typical direct dependence on the time and/or value of poling in forward bias. ${ }^{7,-17}$ Differently to case (a), the RF scans have no symmetry with respect to the steady state curve and are quite dependent on the time and/or value of reverse polarization.

(c) Apparent photocurrent enhancement in FR direction. When increasing the scan rate $b$, a plateau current is achieved (as in normal diode behaviour) that increases significantly the final photocurrent with increasing scan rate. ${ }^{7,18-20}$ Here, $j_{s c}$ is modified while the low forward bias resistance and $V_{o c}$ are unaltered. Unlike (a) and similarly to (b), the RF curves are very sensitive to other experimental parameters and diverse forms can be delivered.

(d) Strong decay of photocurrent in RF direction. This behaviour has been reported ${ }^{19-}$ ${ }^{21}$ as result of specific polarization routines. In a possibly related extreme case, the RF photocurrent achieves the opposite sign. ${ }^{22-23}$

(e) Remarkable ohmic-like behaviour at low forward and reverse applied bias. The current increases in FR direction in an apparent shunt resistance effect, however, it is dependent on bias, scan rate and direction, ${ }^{17-20}$ illumination ${ }^{7,24}$ and temperature. ${ }^{17}$ This feature also modifies the $j_{s c}$.

(f) Apparent $V_{o c}$ shift. The RF measurement delivers a lower $V_{o c}$ than the one for the FR curve. ${ }^{24-26}$ Here, the $j_{s c}$ and the resistance at low forward bias are not modified. Subsequently, a mismatch between initial and final current after a loop may occur. ${ }^{25}$

(g) Crossing between curves at the same scan rate with opposite directions. Here, the RF curve exceeds the FR within some region between short circuit and open circuit, ${ }^{10,24-25}$ unlike all previous patterns where the absolute current is always lower in RF direction than in FR. Furthermore, the crossing can occur at low forward or reverse bias meaning that a larger current is achieved for the RF scan in practically the whole first quadrant (larger FF). ${ }^{27}$

It should be also remarked that hysteretic effects practically disappear at room temperature when fullerene contacts are used instead of $\mathrm{TiO}_{2}$ as electron-transport material. This indicates that ion migration is a necessary but not sufficient condition for interfacial accumulation to occur. The fact that fullerene-based perovskite solar cells do not exhibit significant capacitance in excess of the geometrical value corroborates the interface polarization origin of the hysteresis. ${ }^{28}$ Several papers have explored specific features of the $\mathrm{TiO}_{2}$ /perovskite interface responsible for its charge accumulation ability. Ti-I bonds easily accommodate excess or defect ionic charge in a highly reversible way causing the capacitive currents. ${ }^{29}$ Also the morphology (disorder degree) at the interface has been related to hysteresis reduction signaling that $\mathrm{MAPbI}_{3}$ at the interface should be both homogenous and crystalline. ${ }^{12}$ With respect to the role of anodes (HTL/perovskite contacts), several recent papers have directly 
observed that charge accumulation at the $\mathrm{TiO}_{2}$ interface is larger than that occurring at spiro-OMeTAD/perovskite interfaces. Weber et al. ${ }^{30}$ have shown that the positive space-charge near the $\mathrm{TiO}_{2}$ contact exceeds that at the HTL. We also observed by comparing symmetrical devices of structure $\mathrm{TiO}_{2} /$ perovskite $/ \mathrm{TiO}_{2}$ and spiroOMeTAD/perovskite/spiro-OMeTAD, that it is precisely the $\mathrm{TiO}_{2} /$ perovskite interface that is able to reversibly accumulate charge producing capacitive currents while spiroOMeTAD contacts do not. ${ }^{31}$

\section{Physical effects leading to dynamic hysteresis in perovskite solar cells}

In the literature, there are two main models based on detailed simulation of transportrecombination approach. The model of Snaith et al. ${ }^{32}$ combines ionic drift and surface trapping to explain the pattern in Fig. 1d but this model does not systematically implement dynamic scans. On the other hand, Walker et al. ${ }^{21}$ describe the solar cell as a macrocapacitor where ions can be accumulated at the interface. This model reproduces by this feature the capacitive properties observed in experiments and also describes well the large asymmetry in Fig. 1d, however the electronic features are simplified and the $j-V$ curve is described in terms of charge collection controlled by the electric field. Therefore, the model cannot describe the wide phenomenology commented before.

Our approach in this Letter is to design a simple model that captures the physical core of the dynamic hysteresis without the burden of massive computational cost. In early work, a persistent voltage was observed in photovoltage decays spanning such a long time scale that it was concluded that it cannot be due to electronic recombination alone. ${ }^{33-34}$ Many authors have attributed the slow modification of the cell response to the ion redistribution ${ }^{35-37}$ but the central question is how the ionic effect influences the electronic features that are finally measured in $j-V$ performance. One natural possibility is to relate the ionic redistribution to the modification of charge collection efficiency by the changes of internal electric field in the solar cell. ${ }^{20-21,36}$ However, the lead halide perovskite solar cell has extremely long diffusion length, ${ }^{38-39}$ thus electric field should be a minor factor in the $j-V$ curve, unless in extreme situations. Such type of situation may be produced if the perovskite solar cell is polarized for a substantial period of time at reverse bias, in which case a drift of ions can heavily modify the condition of operation by establishing a pseudo built-in voltage that decreases the charge collection efficiency, as recognized by Tress et al. ${ }^{20}$ and Walker et al. ${ }^{21}$ In fact, Snaith et al. ${ }^{32}$ have nicely shown that the feature of Fig. 1d becomes more pronounced when the poling voltage becomes progressively more reverse. Under exaggerated pre-treatment, the contacts can be severely modified so that the solar cell can be forced to produce negative values of the photocurrent. ${ }^{23}$ However, we show in Fig. SIld that one can also observe the characteristic features of dynamic hysteresis in the dark, which therefore cannot be primarily due to changes of charge collection properties. It has also been shown ${ }^{14}$ that if a scan goes in RF direction at successive voltage steps without pre-treatment at $\mathrm{R}$, and then FR, only the instantaneous capacitive behaviour with a symmetric current around steady state value is 
observed, as in Fig. 1a. Thus, the spurious reverse pre-polarization effects can be avoided. Therefore, we leave these phenomena arising from sustained forcing of the cell at $\mathrm{R}$ bias out of the scope of our model in which we will analyse the FR scan predominantly.

\section{Evidence from capacitance results for surface polarization}

Our model is based on the observation that in F polarization, the interface can be charged electronically (with electrons and holes at either side of the interface), which creates a significant transient current that causes strong hysteresis effect in a FR scan measurement. This picture is consistent with several physical observations on the operation of hybrid perovskite solar cells. ${ }^{19}, 26,30,40-42$ As we know from plenty of evidence, the interfaces can be affected by strong electronic charging assisted by the presence of mobile ions. ${ }^{15,37,40-41}$ The meaning of capacitance vs. frequency has been well understood. ${ }^{43-44}$ The low frequency large capacitance in the dark is associated to the ionic polarization at the perovskite interface with the contact while the higher frequency plateau is a dielectric polarization in the bulk of the perovskite. ${ }^{15,45} \mathrm{An}$ interpretation of the huge capacitance observed under illumination was suggested by Zarazua et al. in terms of accumulation of majority carriers at the interface. ${ }^{40}$ They provided a picture of an electrostatic component to the photovoltage, produced by upward band-bending at the electron contact. Gottesmann et al. showed that the electrostatic photovoltage can explain long time transients in open circuit voltage decay. ${ }^{41}$ This observation does not rule out the presence of interfacial electronic states at the contact that may produce slow kinetic effects. But as shown later, the charge stored is so large that traps cannot explain this effect, therefore trapping of carriers is not a primary consideration in our model.

However, why is the kinetics of the large capacitance very slow? This question was analysed with a study of photovoltage decay as a function of illumination preconditioning. ${ }^{41}$ By combining the analysis of decays and detailed simulation of the interface, it was found that accumulation of cations at the interface assists the formation of the electronic accumulation. Previously, the decay of external voltage was described by the relaxation of voltage. ${ }^{34}$ Pintillie et al. ${ }^{19}$ have provided a model description of the dynamic hysteresis under cycling in terms of internal depolarization, but their model is for a dielectric relaxation. Weber et al. identified in situ the distribution of space charge across the absorber layer, situated at the contact interfaces. ${ }^{30}$ A correlation between interfacial capacitance and the amount of hysteresis was realized, ${ }^{27,46}$ and especially the $\mathrm{TiO}_{2}$ electron contact displays an enormous capacitance combined with a large hysteresis effect.

According to the previous observations, the interface changes dramatically with the voltage, producing a huge accumulation of charge, with the kinetics controlled by ion diffusion. Interface recombination is a major impediment to charge collection. The diffusion of ions and its influence on the global electrical field appear to be of secondary interest, rather it is the accumulation of ions at the interface and their rate of arrival and departure from there being the central effect governing the operational properties of the 
cell.

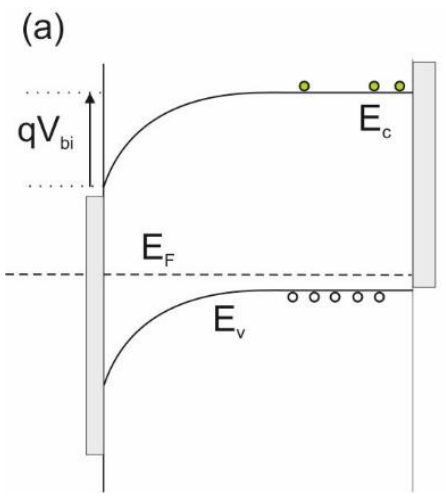

(b)

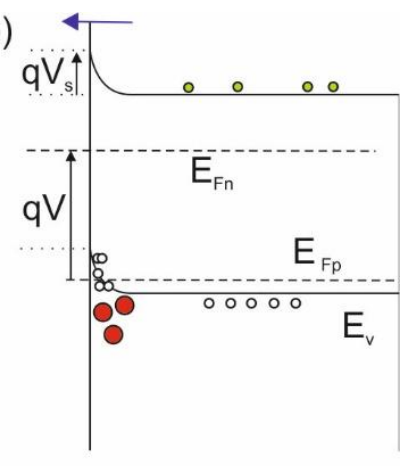

Fig. 2. Schematic representation of the energy diagram of a perovskite solar cell with electron selective contact at the left and hole selective contact at the right side. (a) equilibrium in the dark. (b) Open circuit under illumination. $E_{c}, E_{V}$ : Edges of conduction and valence band $E_{F}$ : Fermi Level $, E_{F p}: E_{F n}$ quasi-Fermi level of electrons and holes. $V$ : external voltage of the contacts. $V_{b i}$ : the constant built-in voltage. $V_{s}$ : The variable surface polarization voltage. Indicated in (b) are the accumulation of holes and cations and the tunnelling of electrons across the surface barrier. Note that electrons at the contact side of the interface that contribute to charge compensation are not shown.

\section{Surface polarization model of dynamic hysteresis}

The description of $j-V$ curve measurement results in Fig. 1 requires a model for the current obtained under illumination when we sweep the voltage at rate $b$. We now describe such a model based on the diagrams of Fig. 2. The main assumption adopted here is a distinction of the external voltage $V$ and the surface polarization voltage $V_{s}$. The former corresponds to the voltage at the contacts which can be changed instantaneously, while $V_{s}$ is an internal voltage by upward bending at the surface that responds by internal kinetics and determines the excess charge density of holes at the interface. ${ }^{40-41}$ In a first approximation, the surface charge is

$$
Q_{s}=q p_{s}=Q_{s 0} e^{q V_{s} / 2 k_{B} T}
$$

in terms of surface hole density $p_{s}$, the elementary electrical charge $q$, the thermal energy $k_{B} T$ and a general exponent $\gamma$ that in the ideal case takes the value 2 . The accumulation capacitance is

$$
C_{a c c}=\frac{d Q_{s}}{d V_{s}}=\frac{q Q_{s 0}}{\gamma k_{B} T} e^{q V_{s} / \gamma_{B} T} .
$$

The surface charge parameter can be estimated from the following expression, ${ }^{40}$ where $p_{0}$ is the equilibrium hole density

$$
Q_{s 0}=\sqrt{2} q p_{0} L_{D}
$$

and $L_{D}$ is the Debye length 


$$
L_{D}=\sqrt{\varepsilon \varepsilon_{0} k_{\mathrm{B}} T / q^{2} p_{0}} .
$$

At steady state condition at a given voltage $V$, the surface polarization depends on the built-in potential $V_{b i}$ as follows

$$
V_{s}=V-V_{b i} \text {. }
$$

However, as described before, the interfacial charge distribution is strongly determined by the presence of ions, ${ }^{41}$ therefore $V_{s}$ cannot instantaneously follow the external voltage. Considering that the internal voltage $V_{s}$ strives for the equilibrium condition indicated in Eq. (5), we propose a kinetic condition of the type

$$
\frac{d V_{s}}{d t}=-\frac{V_{s}-\left(V-V_{b i}\right)}{\tau_{k i n}}
$$

where the relaxation kinetic constant $\tau_{\text {kin }}$ should be determined by the rapidity of ion displacement at the given illumination and temperature. ${ }^{17,19,34,47}$ Note that Eq. 6 is a central assumption that combines the dynamics and distribution of surface charge (ionic and electronic). A more elaborate model could be based on detailed charge compensation at the surface given by the expression

$$
Q_{s}+q c_{c}=q n_{e t l}
$$

where $c_{c}$ is the surface density of cations, and $n_{e t l}$ is the density of electrons in the outer electron transport layer.

Recombination current at the surface ${ }^{42}$ depends on both electron and hole concentration

$$
j_{\text {rec }}=k_{\text {rec }} n Q_{s}
$$

with a recombination rate constant $k_{\text {rec }}$. The bulk electron density is $n=$ $n_{0} \exp \left(q V / \beta k_{B} T\right)$ where $\beta=1$ is the ideal parameter value. Hence, the recombination current in transient conditions is

$$
j_{r e c}=j_{\text {rec } 0} e^{q V_{b i} / \gamma k_{B} T} e^{q\left(V_{s} / \gamma+V / \beta\right) / k_{B} T},
$$

where the recombination parameter is

$$
j_{r e c 0}=k_{r e c} Q_{s 0} n_{0} e^{-q V_{b i} / \gamma k_{B} T} .
$$

The extracted current is composed of the photocurrent $j_{p h}$ minus the transient recombination current. To these terms, we add the current of extracted excess electrons from the contact layer, which at strong $\mathrm{F}$ bias is $q n_{e t l} \approx Q_{s}$. Hence

$$
j=j_{p h}-j_{r e c 0} e^{q V_{b i} / \gamma k_{B} T} e^{q\left(V_{s} / \gamma+V / \beta\right) / k_{B} T}+\frac{d Q_{s}}{d t} .
$$

Modifying the last term, we obtain

$$
j=j_{p h}-j_{r e c 0} e^{q\left(V_{s} / \gamma+V / \beta+V_{b i} / \gamma\right) / k_{B} T}+C_{a c c} \frac{d V_{s}}{d t} .
$$

The steady state current is obtained by imposing the equilibrium value of surface 
voltage determined by the external potential, Eq. 5. Including the constant dark current in reverse, we obtain the diode equation

$$
j=j_{p h}+j_{\text {rec } 0}\left(e^{q V / m k_{B} T}-1\right)
$$

where

$$
m=\frac{\beta \gamma}{\beta+\gamma} .
$$

The model is complete and allows the calculation of the results of a number of techniques. In this paper, we treat only the usual voltage ramp in the direction FR, that consists of the time dependence

$$
V=V_{\text {in }}+b t
$$

where $V_{\text {in }}$ is the initial voltage and the scan rate $b$ is negative. We assume that the system starts at steady state condition at $V_{\text {in }}$. We also define the initial surface voltage

$$
V_{s 0}=V_{\text {in }}-V_{b i}
$$

(a)

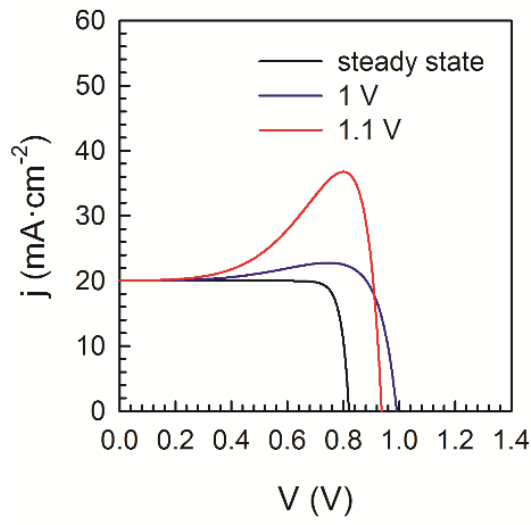

(c)

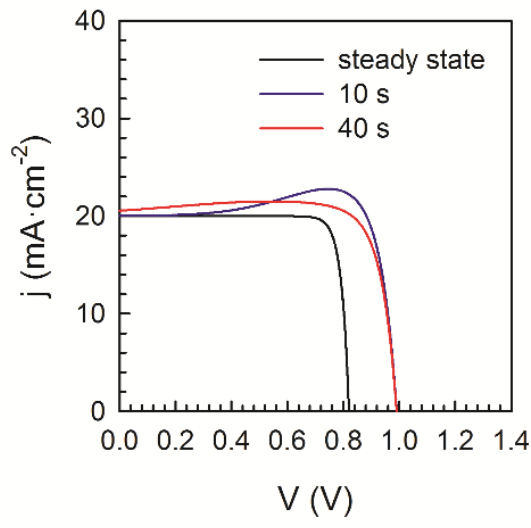

(b)

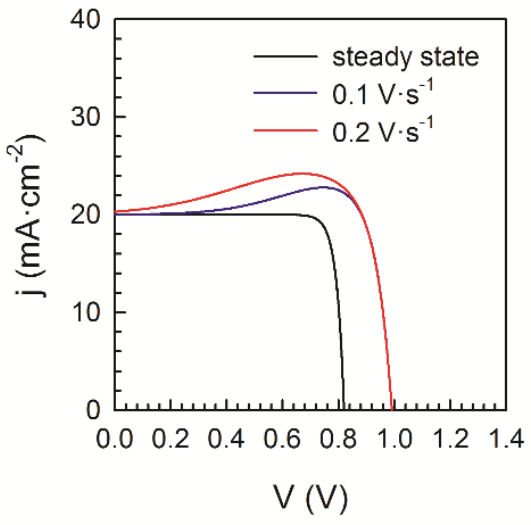

(d)

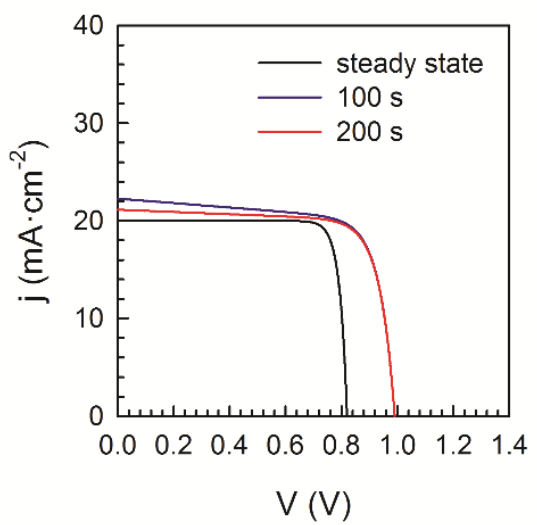

Fig. 3. Simulated FR $j$ - $V$ curves for different (a) initial voltages $\mathrm{V}_{\text {in }}$ (b) scan rates (c) small $\tau_{k i n}$ and (d) large $\tau_{k i n}$ for $b=100 \mathrm{kV} \cdot \mathrm{s}^{-1}$. The parameters for the curves were: $Q_{s 0}=4.4 \times 10^{-8} \mathrm{C} \cdot \mathrm{cm}^{-2}, b=100 \mathrm{mV} \cdot \mathrm{s}^{-1}, V_{b i}=350 \mathrm{mV}, \beta=2, \gamma=2, j_{p h}=20 \mathrm{~mA} \cdot \mathrm{cm}^{-2}, \mathrm{j}_{\mathrm{rec} 0}$ 
$=4.8 \times 10^{-16} \mathrm{~mA} \cdot \mathrm{cm}^{-2}, \tau_{k i n}=10 \mathrm{~s}$ for $(\mathrm{a}),(\mathrm{b})$.

The analytical solution to these conditions is described in the SI. A summary of the pertinent features of the $j$ - $V$ curves simulated from our model is shown in Fig. 3, with a simulated RF scan also shown in Fig. SI3(a). The surface polarization decay yields a "bump" in the current whose magnitude increases with increasing $V_{\text {in }}$ and also with increasing scan rate, as shown in Fig. 3a,b. This "bump" and its evolution is well documented in literature and has already been depicted in Fig. 1b. This "bump" eventually decays down to the photocurrent value depending on the kinetic relaxation time $\tau_{k i n}$. If the kinetic delay time is large enough compared to the scan rate, the obtained short-circuit current value is higher than the maximum photocurrent value, as shown in Fig. 3c. For very large scan rates and large $\tau_{k i n}$ values, we obtain the apparent photocurrent enhancement with the plateau as in a normal diode as described earlier in Fig. $3 \mathrm{~d}$. The slope of this plateau depends inversely on $\tau_{\text {kin }}$, see SI for the mathematical expression. We have also simulated the effect of a reduced accumulation layer at the contacts on the performance of the cell, see Fig. SI2(b).

\section{Model description of experimental results of dynamic hysteresis}

As our model successfully reproduces several of the typical behaviours of FR dynamic hysteresis, we then proceeded to fit some experimental FR $j$ - $V$ curves for $\mathrm{CH}_{3} \mathrm{NH}_{3} \mathrm{PbI}_{3}$ perovskite for different $V_{\text {in }}$ to obtain relevant parameters of the model. We have measured the $j$ - $V$ curves at different forward polarization values which provide increasing values of the "bump" in FR measurement as shown in Fig. 4a. To describe such curves is a challenge since traps at the electron contact should be saturated at large forward bias so that the observed changes cannot be explained only by de-trapping effects. On the other hand, an accumulation capacitance does not have any limitation for additional charging at increasing forward bias as indicated in Eq. 1, and it explains very well the growing current by the release of the accumulated charge.

Here we employed a least square fitting method consisting of a maximum of 7 free parameters, namely $Q_{s 0}, V_{s 0}, \tau_{k i n}, j^{\prime}{ }_{r e c 0}=j_{r e c 0} \exp \left(q V_{b i} / 2 k_{B} T\right), j_{p h}, \beta$ and $\gamma$. The value of $Q_{s 0}$ and $\gamma=2$ was estimated based on the accumulation capacitance of Eqs. 2 and 3. For a doping density of $10^{17} \mathrm{~cm}^{-3}$ and relative dielectric constant $\varepsilon=26$, we obtained $Q_{s} 0$ $=4.4 \times 10^{-8} \mathrm{C} \mathrm{cm}^{-2}$. Subsequent fits of the experimental data yielded an average value for $\beta$ of 6 , that was used to fix this parameter in the model. Note that this means a diode ideality factor value of $m=1.5$, expressed in Eq. 14. The final fitting lines obtained with fixed $Q_{s}, \beta$ and $\gamma$ parameters and their evolution with respect to $V_{i n}$ are shown in Fig 4 and they provide excellent agreement with the data. 

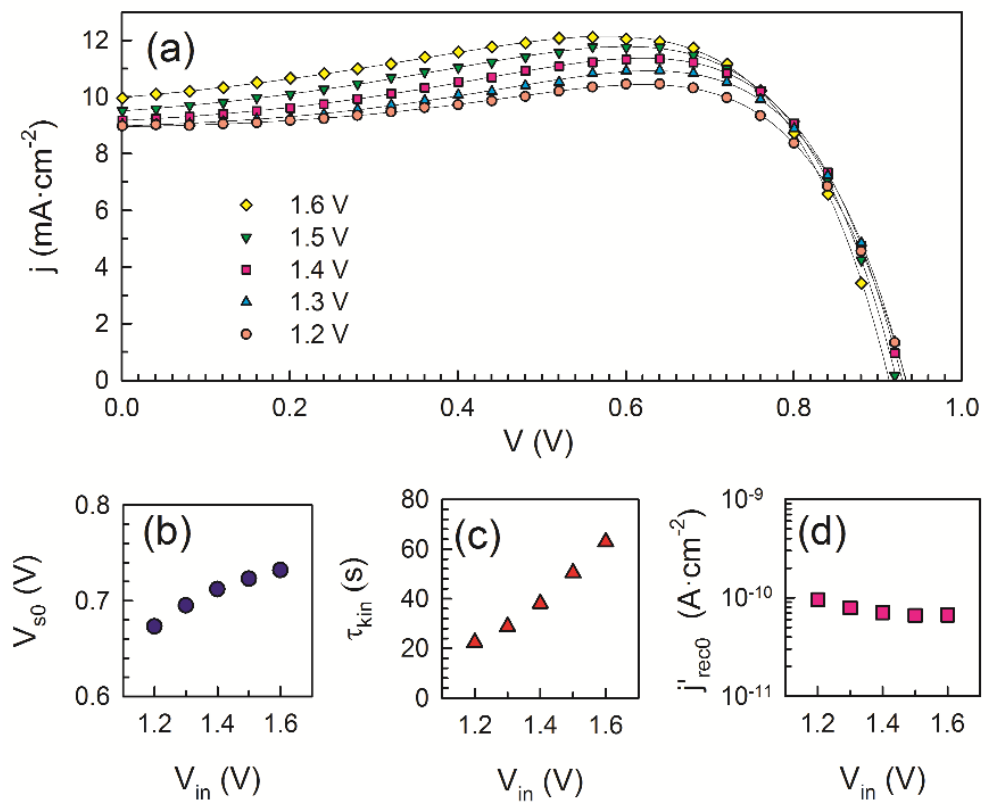

Fig.4. Hysteresis in FR scans of $\mathrm{TiO}_{2} / \mathrm{CH}_{3} \mathrm{NH}_{3} \mathrm{PbI}_{3} /$ spiro-OMeTAD solar cell and the fits of the surface polarization model. (a) Experimental FR $j$ - $V$ curves measured at $b=100$ $\mathrm{mV} / \mathrm{s}$ (symbols) and corresponding fitted curves (lines) for different initial applied voltage $V_{i n}$ as indicated. Parameters (b) $V_{s 0}$ (c) $\tau_{k i n}$ and (d) $j_{r e c 0} \exp \left(q V_{b i} / 2 k_{B} T\right)$ obtained from least square fitting.

We observe in Fig. $4 \mathrm{~b}$ that the initial surface voltage $V_{s 0}$ increases sluggishly with the applied voltage as $V_{s 0} \approx V_{i n} / 2$. Given that $V_{b i}$ in Eq. 16 is a constant, this discrepancy could be related to the fact that the accumulation at the interface depends strongly on several factors such as poling time, the kinetic relaxation time of the ions and the interfacial properties of the contact, leading to deviations from our simplified model. A more complete model using detailed simulation of the interface incorporating Eq. 7 could account for these variations, nevertheless we consider that our simplified model gives an excellent physical description of the charge accumulation and its release, explaining the dynamic hysteresis in FR scan. The suitability of our model for dynamic hysteresis is further confirmed by the fact that the accumulation charge calculated from Eq. 1 and that obtained by integrating the transient part of the $j-V$ curves closely match; the corresponding charge values are given in Fig. SI3. The kinetic relaxation time itself evolves linearly with $V_{i n}$, which is expected, as the large upward band bending of the accumulation layer, which increases with $V_{i n}$, opposes the flow of cations away from the interface. These obtained parameters can therefore serve as a good reference point for understanding the nature of the accumulation at the interface and recombination in perovskite solar cells, while providing useful information about internal parameters of the cell in a rather simplistic manner.

In conclusion, we have developed a dynamic hysteresis model based on the charging 
of the contact interface by the formation of an accumulation layer of holes and ions compensated by electrons at the contact. This surface voltage strives for the equilibrium condition based on a kinetic relaxation time of displacement of the ions, leading to a discharge current upon cycling the voltage. By including surface recombination, we reproduce many of the observed cases of experimental hysteresis in perovskites depending on experimental parameters such as the poling voltage and the scan rate. The model provides a quantitative explanation of different typical hysteretic features that occur in measurements from forward to reverse voltage scans. Our results highlight that hysteresis can be explained by ionic movement and electrode polarization exclusively without the effect of trap assisted recombination. The detailed analytical equations establish for the first time the possibility to systematically investigate quantitatively the effect of previous conditions applied to the solar cell. Furthermore, the model is consistent with other methods such as impedance spectroscopy, open circuit voltage decays and Kelvin probe methods, which opens the opportunity to investigate by different independent methodologies the peculiar kinetic phenomena widely observed in perovskite solar cells.

\section{ASSOCIATED CONTENT}

Supporting Information

The Supporting Information is available free of charge on the

ACS Publications website at

Preparation methods; Features of current-voltage curves; Analytical solutions of $\mathrm{j}-\mathrm{V}$ curves; Accumulated charge at the interface

\section{AUTHOR INFORMATION}

Corresponding Author

*E-mail: bisquert@uji.es (J.B.).

ORCID

Juan Bisquert: 0000-0003-4987-4887

Notes

The authors declare no competing financial interest.

\section{Acknowledgements}

We acknowledge funding from MINECO of Spain under Project MAT2016-76892-C31-R and Generalitat Valenciana Project PROMETEOII/2014/020. S. R. and O. A. acknowledge Generalitat Valenciana for the grants (GRISOLIAP2014/034) and (GRISOLIA/2014/035) respectively. 


\section{References}

1. Kojima, A.; Teshima, K.; Shirai, Y.; Miyasaka, T. Organometal Halide Perovskites as Visible-Light Sensitizers for Photovoltaic Cells. Journal of the American Chemical Society 2009, 131 (17), 6050-6051.

2. Park, N.-G.; Grätzel, M.; Miyasaka, T. Organic-Inorganic Halide Perovskite Photovoltaics: From Fundamentals to Device Architectures. 1 ed.; Springer: 2016; p VIII, 366.

3. Lee, M. M.; Teuscher, J.; Miyasaka, T.; Murakami, T. N.; Snaith, H. J. Efficient Hybrid Solar Cells Based on Meso-Superstructured Organometal Halide Perovskites. Science 2012, 338 (6107), 643-647.

4. Sanchez, R. S.; Gonzalez-Pedro, V.; Lee, J.-W.; Park, N.-G.; Kang, Y. S.; Mora-

Sero, I.; Bisquert, J. Slow Dynamic Processes in Lead Halide Perovskite Solar Cells. Characteristic Times and Hysteresis. The Journal of Physical Chemistry Letters 2014, 5 (13), 2357-2363.

5. Snaith, H. J.; Abate, A.; Ball, J. M.; Eperon, G. E.; Leijtens, T.; Noel, N. K.; Stranks, S. D.; Wang, J. T.-W.; Wojciechowski, K.; Zhang, W. Anomalous Hysteresis in Perovskite Solar Cells. The Journal of Physical Chemistry Letters 2014, 5 (9), 1511-1515.

6. Kim, H.-S.; Park, N.-G. Parameters Affecting I-V Hysteresis of $\mathrm{CH}_{3} \mathrm{NH}_{3} \mathrm{PbI}_{3}$ Perovskite Solar Cells: Effects of Perovskite Crystal Size and Mesoporous $\mathrm{TiO}_{2}$ Layer. The Journal of Physical Chemistry Letters 2014, 5 (17), 2927-2934.

7. Unger, E. L.; Hoke, E. T.; Bailie, C. D.; Nguyen, W. H.; Bowring, A. R.; Heumuller, T.; Christoforo, M. G.; McGehee, M. D. Hysteresis and Transient Behavior in Current-Voltage Measurements of Hybrid-Perovskite Absorber Solar Cells. Energy \& Environmental Science 2014, 7 (11), 3690-3698.

8. Christians, J. A.; Manser, J. S.; Kamat, P. V. Best Practices in Perovskite Solar Cell Efficiency Measurements. Avoiding the Error of Making Bad Cells Look Good. The Journal of Physical Chemistry Letters 2015, 6 (5), 852-857.

9. Zimmermann, E.; Wong, K. K.; Müller, M.; Hu, H.; Ehrenreich, P.; Kohlstädt, M.; Würfel, U.; Mastroianni, S.; Mathiazhagan, G.; Hinsch, A.; Gujar, T. P.; Thelakkat, M.; Pfadler, T.; Schmidt-Mende, L. Characterization of Perovskite Solar Cells: Towards a Reliable Measurement Protocol. APL Materials 2016, 4 (9), 091901.

10. Almora, O.; Aranda, C.; Zarazua, I.; Guerrero, A.; Garcia-Belmonte, G. Noncapacitive Hysteresis in Perovskite Solar Cells at Room Temperature. ACS Energy Letters 2016, 1, 209-215.

11. Domanski, K.; Roose, B.; Matsui, T.; Saliba, M.; Turren-Cruz, S.-H.; CorreaBaena, J.-P.; Carmona, C. R.; Richardson, G.; Foster, J. M.; De Angelis, F.; Ball, J. M.; Petrozza, A.; Mine, N.; Nazeeruddin, M. K.; Tress, W.; Gratzel, M.; Steiner, U.; Hagfeldt, 
A.; Abate, A. Migration of Cations Induces Reversible Performance Losses over Day/Night Cycling in Perovskite Solar Cells. Energy \& Environmental Science 2017, DOI: $10.1039 / \mathrm{C6EE03352K}$

12. Patel, J. B.; Wong-Leung, J.; Van Reenen, S.; Sakai, N.; Wang, J. T. W.; Parrott, E. S.; Liu, M.; Snaith, H. J.; Herz, L. M.; Johnston, M. B. Influence of Interface Morphology on Hysteresis in Vapor-Deposited Perovskite Solar Cells. Advanced Electronic Materials 2016, 1600470-n/a.

13. Chen, B.; Yang, M.; Zheng, X.; Wu, C.; Li, W.; Yan, Y.; Bisquert, J.; GarciaBelmonte, G.; Zhu, K.; Priya, S. Impact of Capacitive Effect and Ion Migration on the Hysteretic Behavior of Perovskite Solar Cells. The Journal of Physical Chemistry Letters 2015, 6 (23), 4693-4700.

14. Ono, L. K.; Raga, S. R.; Remeika, M.; Winchester, A. J.; Gabe, A.; Qi, Y. PinholeFree Hole Transport Layers Significantly Improve the Stability of $\mathrm{MAPbI}_{3}$-based Perovskite Solar Cells under Operating Conditions. Journal of Materials Chemistry A 2015, 3 (30), 15451-15456.

15. Almora, O.; Zarazua, I.; Mas-Marza, E.; Mora-Sero, I.; Bisquert, J.; GarciaBelmonte, G. Capacitive Dark Currents, Hysteresis, and Electrode Polarization in Lead Halide Perovskite Solar Cells. The Journal of Physical Chemistry Letters 2015, 6 (9), 1645-1652.

16. Valles-Pelarda, M.; Hames, B. C.; García-Benito, I.; Almora, O.; Molina-Ontoria, A.; Sánchez, R. S.; Garcia-Belmonte, G.; Martín, N.; Mora-Sero, I. Analysis of the Hysteresis Behavior of Perovskite Solar Cells with Interfacial Fullerene Self-Assembled Monolayers. The Journal of Physical Chemistry Letters 2016, 7 (22), 4622-4628.

17. Ono, L. K.; Raga, S. R.; Wang, S.; Kato, Y.; Qi, Y. Temperature-Dependent Hysteresis Effects in Perovskite-Based Solar Cells. Journal of Materials Chemistry A 2015, 3 (17), 9074-9080.

18. Correa-Baena, J. P.; Steier, L.; Tress, W.; Saliba, M.; Neutzner, S.; Matsui, T.; Giordano, F.; Jacobsson, T. J.; Srimath Kandada, A. R.; Zakeeruddin, S. M.; Petrozza, A.; Abate, A.; Nazeeruddin, M. K.; Gratzel, M.; Hagfeldt, A. Highly Efficient Planar Perovskite Solar Cells through Band Alignment Engineering. Energy \& Environmental Science 2015, 8 (10), 2928-2934.

19. Nemnes, G. A.; Besleaga, C.; Tomulescu, A. G.; Pintilie, I.; Pintilie, L.; Torfason, K.; Manolescu, A. Dynamic Electrical Behavior of Halide Perovskite Based Solar Cells. Solar Energy Materials and Solar Cells 2017, 159, 197-203.

20. Tress, W.; Marinova, N.; Moehl, T.; Zakeeruddin, S. M.; Nazeeruddin, M. K.; Gratzel, M. Understanding the Rate-Dependent J-V Hysteresis, Slow Time Component, and Aging in $\mathrm{CH}_{3} \mathrm{NH}_{3} \mathrm{PbI}_{3}$ Perovskite Solar Cells: The Role of a Compensated Electric 
Field. Energy \& Environmental Science 2015, 8 (3), 995-1004.

21. Richardson, G.; O'Kane, S. E. J.; Niemann, R. G.; Peltola, T. A.; Foster, J. M.; Cameron, P. J.; Walker, A. B. Can Slow-Moving Ions Explain Hysteresis in the CurrentVoltage Curves of Perovskite Solar Cells? Energy \& Environmental Science 2016, 9 (4), 1476-1485.

22. Park, N.-G. Perovskite Solar Cells: Switchable Photovoltaics. Nature Materials 2015, 14 (2), 140-141.

23. Xiao, Z.; Yuan, Y.; Shao, Y.; Wang, Q.; Dong, Q.; Bi, C.; Sharma, P.; Gruverman, A.; Huang, J. Giant Switchable Photovoltaic Effect in Organometal Trihalide Perovskite Devices. Nature Materials 2015, 14 (2), 193-198.

24. Chen, B.; Yang, M.; Priya, S.; Zhu, K. Origin of J-V Hysteresis in Perovskite Solar Cells. The Journal of Physical Chemistry Letters 2016, 7 (5), 905-917.

25. Giacomo, G.; Koichi, Y. Zero-Dipole Molecular Organic Cations in Mixed Organic-Inorganic Halide Perovskites: Possible Chemical Solution for the Reported Anomalous Hysteresis in the Current-Voltage Curve Measurements. Nanotechnology 2015, 26 (44), 442001.

26. Contreras, L.; Idigoras, J.; Todinova, A.; Salado, M.; Kazim, S.; Ahmad, S.; Anta, J. A. Specific Cation Interactions as the Cause of Slow Dynamics and Hysteresis in Dye and Perovskite Solar Cells: a Small-Perturbation Study. Physical Chemistry Chemical Physics 2016, 18 (45), 31033-31042.

27. Sepalage, G. A.; Meyer, S.; Pascoe, A.; Scully, A. D.; Huang, F.; Bach, U.; Cheng, Y.-B.; Spiccia, L. Copper(I) Iodide as Hole-Conductor in Planar Perovskite Solar Cells: Probing the Origin of J-V Hysteresis. Advanced Functional Materials 2015, 25 (35), $5650-5661$.

28. Garcia-Belmonte, G.; Bisquert, J. Distinction between Capacitive and Noncapacitive Hysteretic Currents in Operation and Degradation of Perovskite Solar Cells. ACS Energy Letters 2016, 1 (4), 683-688.

29. Roiati, V.; Mosconi, E.; Listorti, A.; Colella, S.; Gigli, G.; De Angelis, F. Stark Effect in Perovskite/TiO 2 Solar Cells: Evidence of Local Interfacial Order. Nano Letters 2014, 14 (4), 2168-2174.

30. Bergmann, V. W.; Guo, Y.; Tanaka, H.; Hermes, I. M.; Li, D.; Klasen, A.; Bretschneider, S. A.; Nakamura, E.; Berger, R.; Weber, S. A. L. Local Time-Dependent Charging in a Perovskite Solar Cell. ACS Applied Materials and Interfaces 2016, 8 (30), 19402-19409.

31. Carrillo, J.; Guerrero, A.; Rahimnejad, S.; Almora, O.; Zarazua, I.; Mas-Marza, E.; Bisquert, J.; Garcia-Belmonte, G. Ionic Reactivity at Contacts and Aging of Methylammonium Lead Triiodide Perovskite Solar Cells. Advanced Energy Materials 
2016, 6 (9), 1502246.

32. van Reenen, S.; Kemerink, M.; Snaith, H. J. Modeling Anomalous Hysteresis in Perovskite Solar Cells. The Journal of Physical Chemistry Letters 2015, 6 (19), 38083814.

33. Baumann, A.; Tvingstedt, K.; Heiber, M. C.; Väth, S.; Momblona, C.; Bolink, H. J.; Dyakonov, V. Persistent Photovoltage in Methylammonium Lead Iodide Perovskite Solar Cells. APL Materials 2014, 2 (8), 081501.

34. Bertoluzzi, L.; Sanchez, R. S.; Liu, L.; Lee, J.-W.; Mas-Marza, E.; Han, H.; Park, N.-G.; Mora-Sero, I.; Bisquert, J. Cooperative Kinetics of Depolarization in $\mathrm{CH}_{3} \mathrm{NH}_{3} \mathrm{PbI}_{3}$ Perovskite Solar Cells. Energy \& Environmental Science 2015, 8 (3), 910-915.

35. Azpiroz, J. M.; Mosconi, E.; Bisquert, J.; De Angelis, F. Defects Migration in Methylammonium Lead Iodide and their Role in Perovskite Solar Cells Operation. Energy \& Environmental Science 2015, 8 (7), 2118-2127

36. Eames, C.; Frost, J. M.; Barnes, P. R. F.; O/'Regan, B. C.; Walsh, A.; Islam, M. S. Ionic Transport in Hybrid Lead Iodide Perovskite Solar Cells. Nature Communications 2015, 6, 7497.

37. Almora, O.; Guerrero, A.; Garcia-Belmonte, G. Ionic Charging by Local Imbalance at Interfaces in Hybrid Lead Halide Perovskites. Applied Physics Letters 2016, 108 (4), 043903.

38. Stranks, S. D.; Eperon, G. E.; Grancini, G.; Menelaou, C.; Alcocer, M. J. P.; Leijtens, T.; Herz, L. M.; Petrozza, A.; Snaith, H. J. Electron-Hole Diffusion Lengths Exceeding 1 Micrometer in an Organometal Trihalide Perovskite Absorber. Science 2013, 342 (6156), 341-344.

39. Xing, G.; Mathews, N.; Sun, S.; Lim, S. S.; Lam, Y. M.; Grätzel, M.; Mhaisalkar, S.; Sum, T. C. Long-Range Balanced Electron- and Hole-Transport Lengths in OrganicInorganic $\mathrm{CH}_{3} \mathrm{NH}_{3} \mathrm{PbI}_{3}$. Science 2013, 342 (6156), 344-347.

40. Zarazua, I.; Bisquert, J.; Garcia-Belmonte, G. Light-Induced Space-Charge Accumulation Zone as Photovoltaic Mechanism in Perovskite Solar Cells. The Journal of Physical Chemistry Letters 2016, 7 (3), 525-528.

41. Gottesman, R.; Lopez-Varo, P.; Gouda, L.; Jimenez-Tejada, Juan A.; Hu, J.; Tirosh, S.; Zaban, A.; Bisquert, J. Dynamic Phenomena at Perovskite/Electron-Selective Contact Interface as Interpreted from Photovoltage Decays. Chem 2016, 1 (5), 776-789.

42. Guerrero, A.; Garcia-Belmonte, G.; Mora-Sero, I.; Bisquert, J.; Kang, Y. S.; Jacobsson, T. J.; Correa-Baena, J.-P.; Hagfeldt, A. Properties of Contact and Bulk Impedances in Hybrid Lead Halide Perovskite Solar Cells Including Inductive Loop Elements. The Journal of Physical Chemistry C 2016, 120 (15), 8023-8032.

43. Bisquert, J.; Garcia-Belmonte, G.; Mora-Sero, I. Characterization of Capacitance, 
Transport and Recombination Parameters in Hybrid Perovskite and Organic Solar Cells. In Unconventional Thin Film Photovoltaics, Como, E. D.; Angelis, F. D.; Snaith, H.; Walker, A., Eds. The Royal Society of Chemistry: London, 2016.

44. Bisquert, J.; Garcia-Belmonte, G.; Guerrero, A. Impedance Characteristics of Hybrid Organometal Halide Perovskite Solar Cells. In Organic-Inorganic Halide Perovskite Photovoltaics: From Fundamentals to Device Architectures, Park, N.-G.; Grätzel, M.; Miyasaka, T., Eds. Springer: 2016.

45. Almora, O.; Aranda, C.; Mas-Marzá, E.; Garcia-Belmonte, G. On Mott-Schottky Analysis Interpretation of Capacitance Measurements in Organometal Perovskite Solar Cells. Applied Physics Letters 2016, 109 (17), 173903.

46. Kim, H.-S.; Jang, I.-H.; Ahn, N.; Choi, M.; Guerrero, A.; Bisquert, J.; Park, N.-G. Control of I-V Hysteresis in $\mathrm{CH}_{3} \mathrm{NH}_{3} \mathrm{PbI}_{3}$ Perovskite Solar Cell. The Journal of Physical Chemistry Letters 2015, 6 (22), 4633-4639.

47. Bryant, D.; Wheeler, S.; O’Regan, B. C.; Watson, T.; Barnes, P. R. F.; Worsley, D.; Durrant, J. Observable Hysteresis at Low Temperature in "Hysteresis Free" OrganicInorganic Lead Halide Perovskite Solar Cells. The Journal of Physical Chemistry Letters 2015, 6 (16), 3190-3194. 


\title{
Surface Polarization Model for the Dynamic Hysteresis of Perovskite Solar Cells
}

\author{
Sandheep Ravishankar, ${ }^{1}$ Osbel Almora, ${ }^{1}$ Carlos Echeverría-Arrondo, ${ }^{1}$ Elnaz \\ Ghahremanirad, ${ }^{1,2}$ Clara Aranda, ${ }^{1}$ Antonio Guerrero, ${ }^{1}$ Francisco Fabregat- \\ Santiago, ${ }^{1}$ Arie Zaban, ${ }^{3}$ Germà Garcia-Belmonte, ${ }^{1}$ Juan Bisquert ${ }^{1 *}$
}

${ }^{1}$ Institute of Advanced Materials (INAM), Universitat Jaume I, 12006 Castelló, Spain

${ }^{2}$ Nano-photonics and Optoelectronics Research Laboratory (NORLab), Shahid Rajaee Teacher Training University, 16788- 15811, Lavizan, Tehran, Iran

${ }^{3}$ Department of Chemistry, Institute for Nanotechnology \& Advanced Materials, BarIlan University, Ramat Gan 52900, Israel.

Email: bisquert@uji.es

\section{Preparation method}

FTO/TiO 2 (compact) $/ \mathrm{TiO}_{2}$ (mesoporous) $/ \mathrm{CH}_{3} \mathrm{NH}_{3} \mathrm{PbI}_{3} /$ spiro-OMeTAD/Au cells were prepared over FTO coated glasses $(25 \times 25 \mathrm{~mm}$, Pilkington TEC15, $\sim 15 \Omega /$ sq resistance $)$, which were partially etched with zinc powder and $\mathrm{HCl}(2 \mathrm{M})$ in order to avoid short circuits, obtaining $0.25 \mathrm{~cm}^{2}$ of active electrode area. The substrates were cleaned with soap (Hellmanex) and rinsed with Milli-Q water and ethanol. Then, the sheets were sonicated for $15 \mathrm{~min}$ in a solution of acetone:isopropanol $(1: 1 \mathrm{v} / \mathrm{v})$, rinsed with ethanol, and dried with compressed air. After that, the substrates were treated in a $\mathrm{UV}-\mathrm{O}_{3}$ chamber for $10 \mathrm{~min}$.

For the growth of the electron selective contact, a compact $\mathrm{TiO}_{2}$ blocking layer was deposited onto the substrates by spray pyrolysis at $450{ }^{\circ} \mathrm{C}$, using a titanium diisopropoxidebis(acetylacetonate) (75\% in isopropanol, Sigma-Aldrich) solution diluted in absolute ethanol $(0.2 \mathrm{M})$, with oxygen as carrier gas. The spray was performed in 3 steps of $6 \mathrm{~s}$, spraying a total of $5 \mathrm{~mL}$ (approx.), and waiting $30 \mathrm{~s}$ between steps. After the spraying process, the films were kept at $450{ }^{\circ} \mathrm{C}$ for $30 \mathrm{~min}$.

Subsequently, the mesoporous $\mathrm{TiO}_{2}$ layer was deposited by spin coating $100 \mu \mathrm{L}$ at $2000 \mathrm{rpm}$ during $10 \mathrm{~s}$ using a $\mathrm{TiO}_{2}$ paste (Dyesol 30NRd, $30 \mathrm{~nm}$ average particle size) diluted in ethanol (1:5, weight ratio). After drying at $100{ }^{\circ} \mathrm{C}$ for $10 \mathrm{~min}$, the $\mathrm{TiO}_{2}$ mesoporous layer was heated at $500{ }^{\circ} \mathrm{C}$ for $30 \mathrm{~min}$ and later cooled to room temperature. Samples were transferred to a nitrogen filled glovebox equiped with a thermal evaporator.

The perovskite precursor solution was prepared following the process reported by Ahn et al.. ${ }^{1}$ A $50 \mu \mathrm{L}$ perovskite solution containing MAI (Dyesol), PbI2 (TCI, 99.999\%) and DMSO (1:1:1 mol \%) in a DMF solution (50 wt \%) was spin coated at $4000 \mathrm{rpm}$ during 50 seconds with acceleration of $4000 \mathrm{rpm}$. Non-polar diethyl ether was poured onto the spinning substrate after the initial 5-7 s to selectively wash the DMF. A transparent film 
generated after the spin coating and a heat treatment at $65^{\circ} \mathrm{C}$ for 1 min was effective to remove the DMSO. Hence shiny and dark brown $\mathrm{MAPbI}_{3}$ films were obtained by further annealing at $100^{\circ} \mathrm{C}$ for 2 minutes.

For the hole selective contact the perovskite films were covered with $50 \mu \mathrm{L}$ of spiroOMeTAD solution spin coated at $4000 \mathrm{rpm}$ for $30 \mathrm{~s}$ under nitrogen conditions. The spiroOMeTAD solution was prepared by dissolving in $1 \mathrm{~mL}$ of chlorobenzene $72.3 \mathrm{mg}$ of (2,2',7,7'-tetrakis(N,N'-di-p-methoxyphenylamine)-9,9'-spirobifluorene), $28.8 \mu \mathrm{L}$ of 4tert-butylpyridine, and $17.5 \mu \mathrm{L}$ of a stock solution of $520 \mathrm{mg} / \mathrm{mL}$ of lithium bis(trifluoromethylsulfonyl)imide in acetonitrile.

Finally, $60 \mathrm{~nm}$ of gold was thermally evaporated on top of the device to form the electrode contacts using a commercial Univex 250 chamber, from Oerlikon Leybold Vacuum. Before beginning the evaporation, the chamber was evacuated until pressure of $2 \times 10^{-6}$ mbar. The active electrode area of $0.25 \mathrm{~cm}^{2}$ per pixel lied defined by the FTO and the Au contacts. This recipe has been earlier described in previous works. ${ }^{2-3}$

\section{Features of current-voltage curves: literature survey}

Complementary discussion, with respect to the patterns of Fig. 1 of the main text:

(h) Capacitive hysteresis. This behaviour has been earlier identified in other PV technologies ${ }^{4-5}$ and it has been reported, ${ }^{6-8}$ mainly at near steady state (low $b$ values) as also shown in figure SIa in between $75 \mathrm{mV} \cdot \mathrm{s}^{-1}$ and $5 \mathrm{mV} \cdot \mathrm{s}^{-1}$. Specifically in dark $j$ - $V$ curves, several studies ${ }^{2,8-10}$ have reported on the clear linear trend of the additive capacitive currents. Nevertheless, it has been also pointed that this capacitive hysteresis only occurs in perovskite solar cells with $\mathrm{TiO}_{2}$ as electron selective contact, being directly related with the low frequency capacitance increase. $^{2,11}$

(i) Maximum higher than $j_{s c}$ in the elbow of the $j$-V curve when FR scan. This widely reported behaviour in perovskite solar cells ${ }^{8,12-15}$ has been also seeing in other PV technologies ${ }^{16}$ and related with ionic mechanisms. Figures SI1c,d illustrate the characteristic dependence with the forward bias polarization initial value. Interestingly, as shown in figure SI1d, this mechanism also occurs in dark.

(j) Apparent photocurrent enhancement in FR sense. Here we highlight that this commonly reported ${ }^{13-15,17-20}$ feature can be understood and observed as a transition of (b) to (c) when increasing the scan rates. ${ }^{21}$ Figures SIla,c,d display this behaviour.

(k) Strong decay of photocurrent in RF direction. About the several reports ${ }^{14,17,21}$ on this pattern it is worthy to pay special attention on the singular conditions. As with the possibly related extreme giant photovoltaic effect, ${ }^{22-24}$ this is not an usual pattern found just by measuring the $j$ - $V$ curve when testing the device efficiency. Specific routines or pre-treatments are needed for this case, as shown in figures 
SI1a,b.

(l) Ohmic-like behaviour at low $F$ and $R$ applied bias. This shunt resistance $\left(R_{s h}\right)$ lowering effect and its parameters dependency (bias, scan rate and sense, ${ }^{12-14,17,25}$ illumination $^{10,15,19,26-27}$ and temperature ${ }^{12,28-29}$ ) occurs in two possible cases. In a first situation, like figure SI1a, it seems that the actual steady state $j$ - $V$ curve $(b$ less than $75 \mathrm{mV} \cdot \mathrm{s}^{-1}$ ) present low $R_{s h}$ and "good" $j$ - $V$ curves (higher $j_{s c}$ and FF) are only artefacts of the measurements due to the hysteresis at large $b$. On the other hand, as observable in figure SI1b, this low $R_{s h}$-like behaviour can be also obtained in the RF scan sense subsequently after a FR sweep at a particular scan rate and bias window.

(m)Apparent $V_{\text {oc }}$ shift. This feature is among the most reported patterns ${ }^{18-20,25,28,30-31}$ and it is also illustrated in figure SI1b. The mismatch between initial and final current after the loop ${ }^{20}$ is also pointed in figure SI1b. However it persist in other patterns, as signalled in figure SI1c, where extra poling is used. Interestingly, the $V_{o c}$ shift seems to have no direct dependence on the light absorption since a mere vertical displacement of dark $j-V$ curves of figure SI1d may result in a similar trend (figures $1 \mathrm{f}$ and SI1b).

(n) Crossing between curves at the same scan rate with opposite senses. The several reported crossings ${ }^{19-20,25,32-35}$ have been found not only to occur at low forward or reverse bias with the consequent larger current for the RF scan in practically the whole first quadrant (larger FF). ${ }^{35-36}$ Singularly, multiple crossing directly on the "elbow" of $j-V$ curves have been also reported. ${ }^{37}$ On the other hand, when the crossing lies beyond the $V_{o c}$, it is usually overlooked and the patterns from (a) to (f) may be obtained. This later situation is pointed in figure SI1c.

\section{Features of current-voltage curves: experimental results}

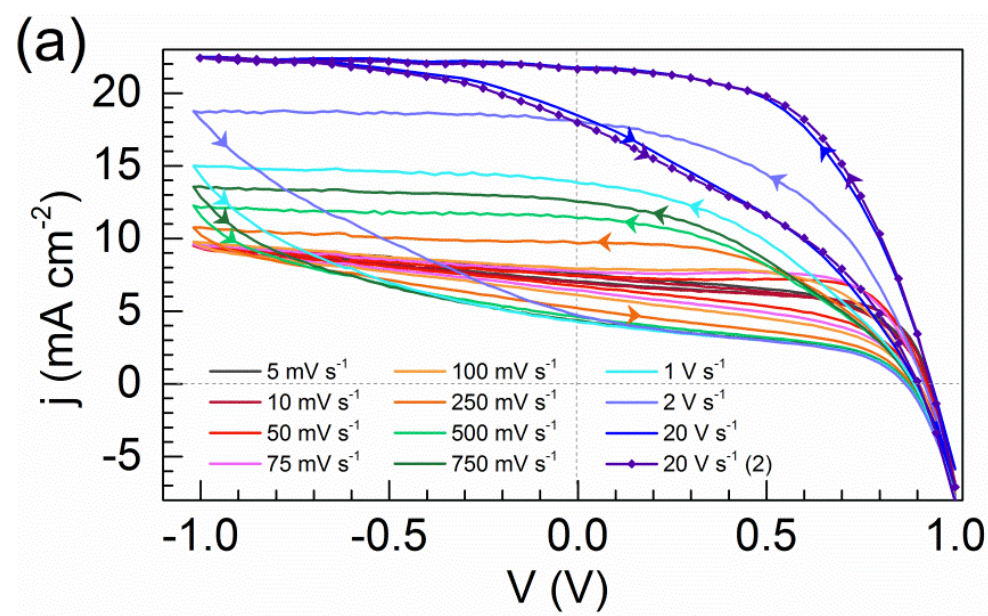



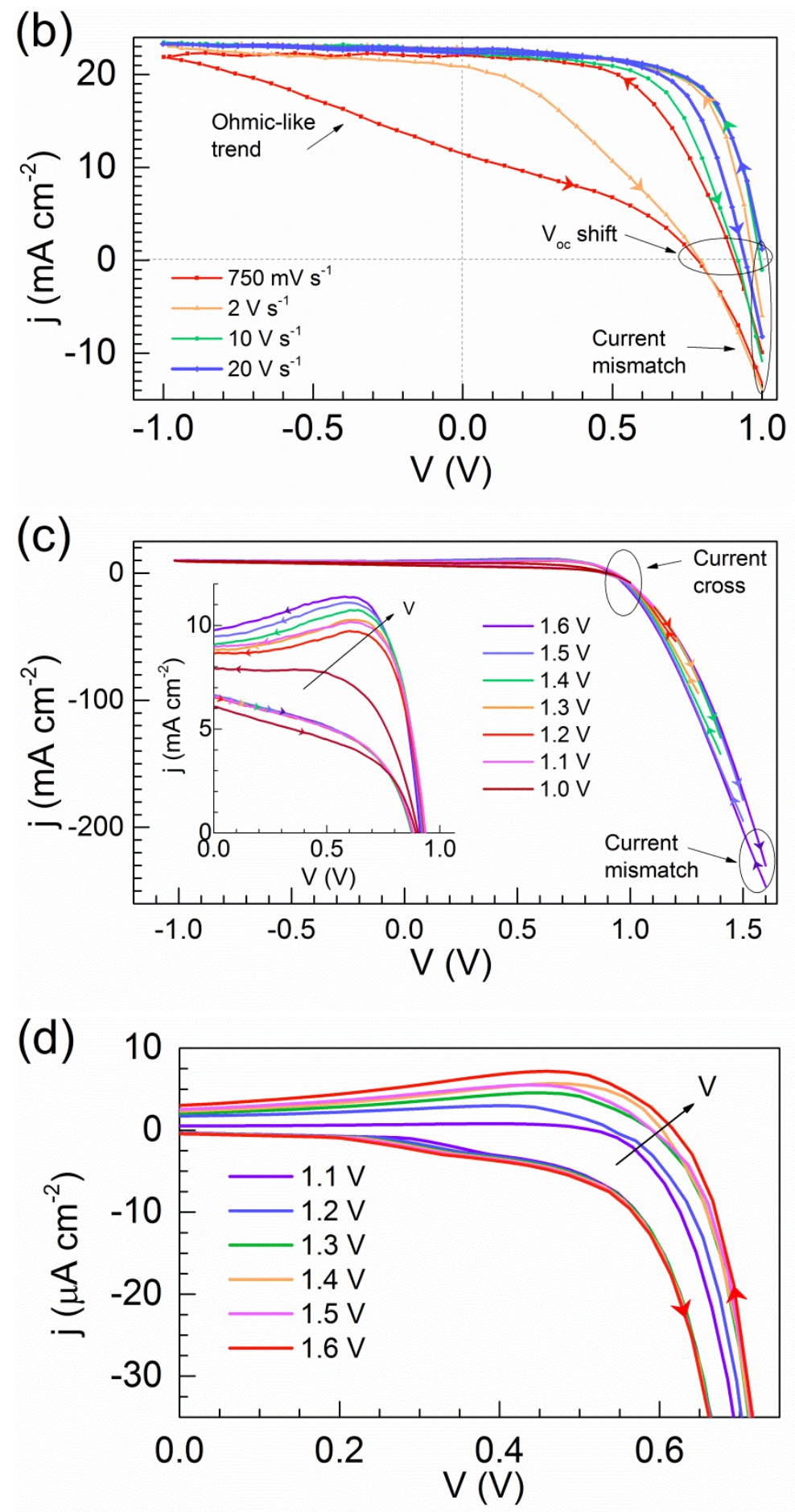

Figure SI1: Experimental current density-voltage curves of FTO/TiO 2 (compact)/TiO 2 (mesoporous) $/ \mathrm{CH}_{3} \mathrm{NH}_{3} \mathrm{PbI}_{3} /$ spiro-OMeTAD/Au devices at room temperature and atmosphere (20-40\% humidity). For (a, b, c) a one sun $\left(100 \mathrm{~mW} \mathrm{~cm}^{-2}\right)$ AM1.5G illumination was used and different $(a, b)$ scan rates and (c) initial forward bias $\left(100 \mathrm{mV} \mathrm{s}^{-1}\right)$ were explored, as indicated. For (c) dark condition at different initial forward bias $\left(500 \mathrm{mV} \mathrm{s}^{-1}\right)$ was considered, as specified. In all cases the colored arrows signals the bias scan sense. In (a) the scan 
rates were checked from the fastest $\left(20 \mathrm{~V} \mathrm{~s}^{-1}\right)$ to the slowest $\left(5 \mathrm{mV} \mathrm{s}^{-1}\right)$, and hence another fast measurement (line and symbols $20 \mathrm{~V} \mathrm{~s}^{-1}$ ) was made for verifying absence of significant degradation effects. The inset in (c) reproduces the region between short circuit and open circuit.

\section{Analytical solutions for $j-V$ curves}

In the proposed model, the total current under constant scan rate is given as a combination of $j_{p h}$ photocurrent, $j_{r e c}$ recombination current, and $j_{c a p}$ capacitor current:

$$
j_{\text {total }}=j_{p h}-j_{\text {rec } 0} e^{\frac{q V_{b i}}{\gamma k_{B} T}} e^{\left(\frac{q V_{s}}{\gamma k_{B} T}+\frac{q V}{\beta k_{B} T}\right)}-\frac{q Q_{s 0}}{\gamma k_{B} T}\left(\frac{d V_{s}}{d t}\right) e^{\frac{q V_{s}}{\gamma k_{B} T}}
$$

The surface polarization potential $V_{s}$ appearing in this expression comes from the solution of Eq. (6):

$$
V_{s}(V)=V-V_{b i}+b \tau_{k i n}\left(1-e^{\left(V-V_{i n}\right) / b \tau_{k i n}}\right)
$$

and since $V=V_{\text {in }}-b t$,

$$
V_{s}(t)=V_{i n}-V_{b i}+b \tau_{k i n}\left(1-e^{-t / \tau_{k i n}}\right)-b t
$$

For large scan rates,

$$
j_{\text {total }}=j_{p h}-j_{\text {rec } 0} e^{\frac{q V_{b i}}{\gamma k_{B} T}} e^{\left(\frac{q V_{s 0}}{\gamma k_{B} T}+\frac{q V}{\beta k_{B} T}\right)}+\frac{q Q_{s 0}}{\gamma k_{B} T}\left(\frac{V_{s 0}-V+V_{b i}}{\tau_{k i n}}\right) e^{\frac{q V_{s 0}}{\gamma k_{B} T}}
$$

and

$$
\frac{d j_{\text {total }}}{d V}=-\frac{q j_{\text {rec } 0}}{\beta k_{B} T} e^{\frac{q V_{b i}}{\gamma k_{B} T}} e^{\left(\frac{q V_{s 0}}{\gamma k_{B} T}+\frac{q V}{\beta k_{B} T}\right)}-\frac{q Q_{s 0}}{\tau_{k i n} \gamma k_{B} T} e^{\frac{q V_{s 0}}{\gamma k_{B} T}}
$$

where it is considered that $t \ll \tau_{k i n} \rightarrow e^{-t / \tau_{k i n}} \approx 1-t / \tau_{\text {kin }} \rightarrow V_{s}=V_{i n}-V_{b i}=V_{s 0} \rightarrow$ $d V_{s} / d t=-\left(V_{s 0}-V+V_{b i}\right) / \tau_{k i n}$.

For large scan rates and applied voltages smaller than $\sim 0.6 \mathrm{~V}$, the $j$ - $V$ curves become straight with slopes inversely dependent on $\tau_{k i n}$, since then it turns out that

$$
\frac{d j_{\text {total }}}{d V} \approx-\frac{q Q_{s 0}}{\tau_{k i n} \gamma k_{B} T} e^{\frac{q V_{s 0}}{\gamma k_{B} T}}
$$




\section{Effect of PCBM layer}

(a)

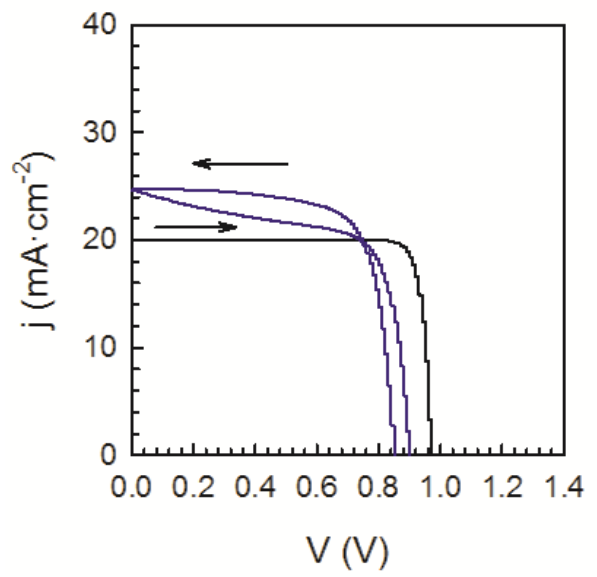

(b)

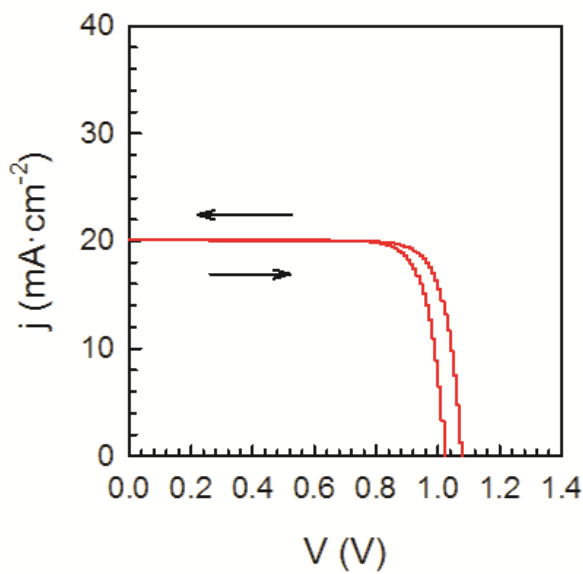

Figure SI2: Simulated $j-V$ curves (a) in both RF and FR direction (black line is the corresponding steady state curve) and (b) by reduction of surface voltage $V_{s 0}$ by a factor 0.75. Parameters for the curves were $-V_{0}-1.1 \mathrm{~V}, \beta=1.9, \gamma=2, V_{b i}=350 \mathrm{mV}, j_{\text {rec } 0}=4.8$ x $10^{-16} \mathrm{~mA} \cdot \mathrm{cm}^{-2}, \tau_{k i n}=200 \mathrm{~s}$

\section{Accumulated charge at the interface}

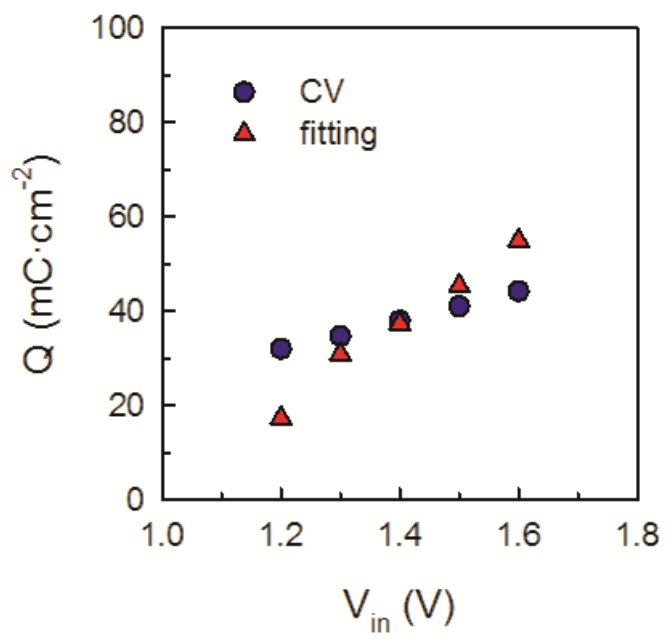

Figure SI3: Total accumulated charge obtained from integration of transient part (subtraction of the steady state curve) of FR $j$ - $V$ curves shown in Fig. 4 from cyclic voltammetry measurements (circles), denoted $\mathrm{CV}$, and from fitting of experimental data with the model (triangles). 


\section{References}

1. Ahn, N.; Son, D.-Y.; Jang, I.-H.; Kang, S. M.; Choi, M.; Park, N.-G. Highly Reproducible Perovskite Solar Cells with Average Efficiency of $18.3 \%$ and Best Efficiency of $19.7 \%$ Fabricated via Lewis Base Adduct of Lead(II) Iodide. Journal of the American Chemical Society 2015, 137 (27), 8696-8699.

2. Almora, O.; Aranda, C.; Zarazua, I.; Guerrero, A.; Garcia-Belmonte, G. Noncapacitive Hysteresis in Perovskite Solar Cells at Room Temperature. ACS Energy Letters 2016, 1 (1), 209-215.

3. Almora, O.; Aranda, C.; Mas-Marzá, E.; Garcia-Belmonte, G. On Mott-Schottky Analysis Interpretation of Capacitance Measurements in Organometal Perovskite Solar Cells. Applied Physics Letters 2016, 109 (17), 173903.

4. Herman, M.; Jankovec, M.; Topic, M. Optimal I-V Curve Scan Time of Solar Cells and Modules in Light of Irradiance Level. International Journal of Photoenergy 2012, $2012,151452$.

5. Albin, D. S.; del Cueto, J. A. In Effect of hysteresis on measurements of thin-film cell performance, Proc. of SPIE 7773, Reliability of Photovoltaic Cells, Modules, Components, and Systems III, San Diego, California, August 18, 2010; Dhere, N. G.; Wohlgemuth, J. H.; Lynn, K., Eds. SPIE-The International Society for Optical Engineering. : San Diego, California, 2010; pp 77730N-1-77730N-9.

6. Chen, B.; Yang, M.; Zheng, X.; Wu, C.; Li, W.; Yan, Y.; Bisquert, J.; GarciaBelmonte, G.; Zhu, K.; Priya, S. Impact of capacitive effect and ion migration on the hysteretic behavior of perovskite solar cells. The Journal of Physical Chemistry Letters 2015, 6, 4693-4700.

7. Ono, L. K.; Raga, S. R.; Remeika, M.; Winchester, A. J.; Gabe, A.; Qi, Y. Pinholefree hole transport layers significantly improve the stability of MAPbI3-based perovskite solar cells under operating conditions. Journal of Materials Chemistry A 2015, 3 (30), 15451-15456.

8. Valles-Pelarda, M.; Hames, B. C.; García-Benito, I.; Almora, O.; Molina-Ontoria, A.; Sánchez, R. S.; Garcia-Belmonte, G.; Martín, N.; Mora-Sero, I. Analysis of the Hysteresis Behavior of Perovskite Solar Cells with Interfacial Fullerene Self-Assembled Monolayers. The Journal of Physical Chemistry Letters 2016, 7 (22), 4622-4628.

9. Almora, O.; Zarazua, I.; Mas-Marza, E.; Mora-Sero, I.; Bisquert, J.; GarciaBelmonte, G. Capacitive Dark Currents, Hysteresis, and Electrode Polarization in Lead Halide Perovskite Solar Cells. The Journal of Physical Chemistry Letters 2015, 6 (9), 1645-1652. 
10. Almora, O. A Characterization of Hybrid Lead Halide Perovskite Solar Cells with $\mathrm{TiO}_{2}$ Mesoporous Scaffold. Universitat Jaume I, Castellón, Spain, 2015.

11. Garcia-Belmonte, G.; Bisquert, J. Distinction between Capacitive and Noncapacitive Hysteretic Currents in Operation and Degradation of Perovskite Solar Cells. ACS Energy Letters 2016, 1 (4), 683-688.

12. Ono, L. K.; Raga, S. R.; Wang, S.; Kato, Y.; Qi, Y. Temperature-dependent hysteresis effects in perovskite-based solar cells. Journal of Materials Chemistry A 2015, 3 (17), 9074-9080.

13. Correa-Baena, J. P.; Steier, L.; Tress, W.; Saliba, M.; Neutzner, S.; Matsui, T.; Giordano, F.; Jacobsson, T. J.; Srimath Kandada, A. R.; Zakeeruddin, S. M.; Petrozza, A.; Abate, A.; Nazeeruddin, M. K.; Gratzel, M.; Hagfeldt, A. Highly efficient planar perovskite solar cells through band alignment engineering. Energy \& Environmental Science 2015, 8, 2928-2934.

14. Nemnes, G. A.; Besleaga, C.; Tomulescu, A. G.; Pintilie, I.; Pintilie, L.; Torfason, K.; Manolescu, A. Dynamic electrical behavior of halide perovskite based solar cells. Solar Energy Materials and Solar Cells 2017, 159, 197-203.

15. Unger, E. L.; Hoke, E. T.; Bailie, C. D.; Nguyen, W. H.; Bowring, A. R.; Heumuller, T.; Christoforo, M. G.; McGehee, M. D. Hysteresis and transient behavior in current-voltage measurements of hybrid-perovskite absorber solar cells. Energy \& Environmental Science 2014, 7 (11), 3690-3698.

16. Contreras, L.; Idigoras, J.; Todinova, A.; Salado, M.; Kazim, S.; Ahmad, S.; Anta, J. A. Specific Cation Interactions as the Cause of Slow Dynamics and Hysteresis in Dye and Perovskite Solar Cells: a Small-Perturbation Study. Physical Chemistry Chemical Physics 2016, 18 (45), 31033-31042.

17. Tress, W.; Marinova, N.; Moehl, T.; Zakeeruddin, S. M.; Nazeeruddin, M. K.; Gratzel, M. Understanding the rate-dependent J-V hysteresis, slow time component, and aging in $\mathrm{CH}_{3} \mathrm{NH}_{3} \mathrm{PbI}_{3}$ perovskite solar cells: the role of a compensated electric field. Energy \& Environmental Science 2015, 8 (3), 995-1004.

18. De Bastiani, M.; Dell'Erba, G.; Gandini, M.; D'Innocenzo, V.; Neutzner, S.; Kandada, A. R. S.; Grancini, G.; Binda, M.; Prato, M.; Ball, J. M.; Caironi, M.; Petrozza, A. Solar Cells: Ion Migration and the Role of Preconditioning Cycles in the Stabilization of the J-V Characteristics of Inverted Hybrid Perovskite Solar Cells. Advanced Energy Materials 2016, 6 (2), n/a-n/a.

19. Chen, B.; Yang, M.; Priya, S.; Zhu, K. Origin of J-V Hysteresis in Perovskite Solar Cells. The Journal of Physical Chemistry Letters 2016, 7 (5), 905-917.

20. Giacomo, G.; Koichi, Y. Zero-dipole molecular organic cations in mixed organicinorganic halide perovskites: possible chemical solution for the reported anomalous 
hysteresis in the current-voltage curve measurements. Nanotechnology 2015, 26 (44), 442001.

21. Richardson, G.; O'Kane, S. E. J.; Niemann, R. G.; Peltola, T. A.; Foster, J. M.; Cameron, P. J.; Walker, A. B. Can slow-moving ions explain hysteresis in the currentvoltage curves of perovskite solar cells? Energy \& Environmental Science 2016, 9 (4), 1476-1485.

22. Park, N.-G. Perovskite solar cells: Switchable photovoltaics. Nature Materials 2015, 14 (2), 140-141.

23. Xiao, Z.; Yuan, Y.; Shao, Y.; Wang, Q.; Dong, Q.; Bi, C.; Sharma, P.; Gruverman, A.; Huang, J. Giant switchable photovoltaic effect in organometal trihalide perovskite devices. Nature Materials 2015, 14 (2), 193-198.

24. Zhao, Y.; Liang, C.; Zhang, H.; Li, D.; Tian, D.; Li, G.; Jing, X.; Zhang, W.; Xiao, W.; Liu, Q.; Zhang, F.; He, Z. Anomalously large interface charge in polarity-switchable photovoltaic devices: an indication of mobile ions in organic-inorganic halide perovskites. Energy \& Environmental Science 2015, 8 (4), 1256-1260.

25. Wei, J.; Zhao, Y.; Li, H.; Li, G.; Pan, J.; Xu, D.; Zhao, Q.; Yu, D. Hysteresis analysis based on the ferroelectric effect in hybrid perovskite solar cells. The Journal of Physical Chemistry Letters 2014, 5 (21), 3937-3945.

26. Zarazua, I.; Han, G.; Boix, P. P.; Mhaisalkar, S.; Fabregat-Santiago, F.; MoraSeró, I.; Bisquert, J.; Garcia-Belmonte, G. Surface Recombination and Collection Efficiency in Perovskite Solar Cells from Impedance Analysis. The Journal of Physical Chemistry Letters 2016, 5105-5113.

27. Rajagopal, A.; Williams, S. T.; Chueh, C.-C.; Jen, A. K. Y. Abnormal CurrentVoltage Hysteresis Induced by Reverse Bias in Organic-Inorganic Hybrid Perovskite Photovoltaics. The Journal of Physical Chemistry Letters 2016, 7 (6), 995-1003.

28. Yu, H.; Lu, H.; Xie, F.; Zhou, S.; Zhao, N. Native Defect-Induced Hysteresis Behavior in Organolead Iodide Perovskite Solar Cells. Advanced Functional Materials 2016, 26 (9), 1411-1419.

29. Bryant, D.; Wheeler, S.; O’Regan, B. C.; Watson, T.; Barnes, P. R. F.; Worsley, D.; Durrant, J. Observable Hysteresis at Low Temperature in "Hysteresis Free" OrganicInorganic Lead Halide Perovskite Solar Cells. The Journal of Physical Chemistry Letters 2015, 6 (16), 3190-3194.

30. Huang, C.; Liu, C.; Di, Y.; Li, W.; Liu, F.; Jiang, L.; Li, J.; Hao, X.; Huang, H. Efficient Planar Perovskite Solar Cells with Reduced Hysteresis and Enhanced Open Circuit Voltage by Using $\mathrm{PW}_{12}-\mathrm{TiO}_{2}$ as Electron Transport Layer. ACS Applied Materials and Interfaces 2016, 8 (13), 8520-8526.

31. Yin, X.; Que, M.; Xing, Y.; Que, W. High efficiency hysteresis-less inverted 
planar heterojunction perovskite solar cells with a solution-derived NiOx hole contact layer. Journal of Materials Chemistry A 2015, 3 (48), 24495-24503.

32. Yang, G.; Tao, H.; Qin, P.; Ke, W.; Fang, G. Recent progress in electron transport layers for efficient perovskite solar cells. Journal of Materials Chemistry A 2016, 4 (11), 3970-3990.

33. Zhang, H.; Liang, C.; Zhao, Y.; Sun, M.; Liu, H.; Liang, J.; Li, D.; Zhang, F.; He, Z. Dynamic interface charge governing the current-voltage hysteresis in perovskite solar cells. Physical Chemistry Chemical Physics 2015, 17 (15), 9613-9618.

34. Wei, Z.; Chen, H.; Yan, K.; Zheng, X.; Yang, S. Hysteresis-free multi-walled carbon nanotube-based perovskite solar cells with a high fill factor. Journal of Materials Chemistry A 2015, 3 (48), 24226-24231.

35. Sepalage, G. A.; Meyer, S.; Pascoe, A.; Scully, A. D.; Huang, F.; Bach, U.; Cheng, Y.-B.; Spiccia, L. Copper(I) Iodide as Hole-Conductor in Planar Perovskite Solar Cells: Probing the Origin of J-V Hysteresis. Advanced Functional Materials 2015, 25 (35), $5650-5661$

36. Nie, W.; Tsai, H.; Asadpour, R.; Blancon, J.-C.; Neukirch, A. J.; Gupta, G.; Crochet, J. J.; Chhowalla, M.; Tretiak, S.; Alam, M. A.; Wang, H.-L.; Mohite, A. D. Highefficiency solution-processed perovskite solar cells with millimeter-scale grains. Science 2015, 347 (6221), 522-525.

37. Tress, W.; Correa Baena, J. P.; Saliba, M.; Abate, A.; Graetzel, M. Inverted Current-Voltage Hysteresis in Mixed Perovskite Solar Cells: Polarization, Energy Barriers, and Defect Recombination. Advanced Energy Materials 2016, 6 (19), n/a-n/a. 


\section{Chapter 5}

\section{Publication 2}

\section{Tunable Hysteresis Effect for Perovskite Solar Cells}

Rong, Y.; Hu, Y.; Ravishankar, S.; Liu, H.; Hou, X.; Sheng, Y.; Mei, A.; Wang, Q.; Li, D.; Xu, M.; Bisquert, J.; Han, H. Tunable hysteresis effect for perovskite solar cells. Energy \& Environmental Science 2017. Reprinted with permission from The Royal Society of Chemistry.

\section{Candidate's contribution}

\begin{tabular}{|l|l|}
\hline Nature of Contribution & Extent of Contribution \\
\hline - & $\begin{array}{l}\text { Proposed experiments to identify } \\
\text { hysteretic mechanisms }\end{array}$ \\
- $\begin{array}{l}\text { Contributed to the development of } \\
\text { the hysteresis model }\end{array}$ & \\
- $\begin{array}{l}\text { Wrote the part of the manuscript } \\
\text { related to the developed model }\end{array}$ & \\
- Contributed to reply to the referees & \\
\hline
\end{tabular}

\section{Introduction}

The surface polarization model (SPM) developed in the previous chapter is extended to account for recombination of the accumulated holes in the perovskite side with the electrons in the $\mathrm{c}-\mathrm{TiO}_{2}$ side, which has a critical dependence on the $\mathrm{c}-\mathrm{TiO}_{2}$ layer thickness. For stable and efficient PSCs, the capacitive discharge is reduced and variations in mainly the $V_{o c}$ and FF are observed. Using the extended SPM, the interplay between charge accumulation and recombination explains the unique $V_{o c}$ hysteresis trends of mesoscopic triple layer architecture PSCs.

\section{Published manuscript}




\title{
Tunable hysteresis effect for perovskite solar cells
}

\author{
Yaoguang Rong ${ }^{\mathrm{a} \dagger}$, Yue $\mathrm{Hu}^{\mathrm{a} \dagger}$, Sandheep Ravishankar ${ }^{\mathrm{b} \dagger}$, Huawei Liu ${ }^{\mathrm{a}}$, Xiaomeng \\ Hou ${ }^{a}$, Yusong Shenga, Anyi Meia, Qifei Wang ${ }^{\mathrm{a}}$, Daiyu $\mathrm{Li}^{\mathrm{a}}$, Mi Xua ${ }^{\mathrm{a}}$, Juan \\ Bisquert $^{\text {b,c } *}$, Hongwei Han ${ }^{\mathrm{a} *}$
}

a Michael Grätzel Center for Mesoscopic Solar Cells, Wuhan National Laboratory for Optoelectronics, School of Optical and Electronic Information, Huazhong University of Science and Technology, Wuhan 430074, Hubei, PR China.

' Institute of Advanced Materials (INAM), Universitat Jaume I, 12006 Castelló, Spain.

'Department of Chemistry, Faculty of Science, King Abdulaziz University, Jeddah, Saudi Arabia.

Email: bisquert@uji.es, hongwei.han@mail.hust.edu.cn

\begin{abstract}
Perovskite solar cells (PSCs) usually suffer an anomalous hysteresis in current-voltage measurements that leads to an inaccurate estimation of the device efficiency. Although ion migration, charge trapping/de-trapping and accumulation have been proposed as a basis for the hysteresis, the origin of hysteresis has not been apparently unraveled. Herein we reported a tunable hysteresis effect based uniquely on open-circuit voltage variations in printable mesoscopic PSCs with a simplified triple-layer $\mathrm{TiO}_{2} / \mathrm{ZrO}_{2} /$ Carbon architecture. The electrons are collected by the compact $\mathrm{TiO}_{2} /$ mesoporous $\mathrm{TiO}_{2}$ (c$\mathrm{TiO}_{2} / \mathrm{mp}-\mathrm{TiO}_{2}$ ) bilayer, and the holes are collected by the carbon layer. By adjusting the spray deposition cycles for the $\mathrm{c}-\mathrm{TiO}_{2}$ layer, we achieved hysteresis-normal, hysteresisfree, and hysteresis-inverted PSCs. Such unique trends of tunable hysteresis are analysed by considering the polarization of the $\mathrm{TiO}_{2}$ /perovskite interface, which can accumulate positive charges reversibly. Successfully tuning the hysteresis effect clarifies the critical importance of the $\mathrm{c}-\mathrm{TiO}_{2} /$ perovskite interface in controlling the hysteretic trends observed, providing important insights towards the understanding of this rapidly developing photovoltaic technology.
\end{abstract}




\section{Introduction}

Recently, organic-inorganic metal halide perovskite solar cells (PSCs) using a methylammonium lead halide $\left(\mathrm{MAPbX}_{3}, \mathrm{X}=\right.$ halogen) and its analogues as the light absorber have rapidly developed. ${ }^{1-3}$ These perovskites have a large absorption coefficient, high carrier mobility, long diffusion length and direct band gap, which make them ideal semiconductors for efficient photovoltaics. ${ }^{4-6}$ Besides, they are comprised of earth-abundant materials and can be deposited by low-temperature solution methods, thus providing a promising prospect for applications. In the past several years, great efforts have been devoted to achieving high efficiency and developing beneficial device fabrication processes, launching the power conversion efficiencies (PCEs) to over $22 \% .{ }^{7}$ At the same time, many attempts to understand the fundamental working principle and mechanism of PSCs have been reported, ${ }^{8-11}$ especially the anomalous current-voltage $(J-V)$ hysteresis behavior. ${ }^{12-}$ 16 This anomalous hysteresis behaviour has been further complicated by the emergence of an 'inverted hysteresis' phenomenon recently. In general, 'normal' hysteresis is the case where there occurs a large loss of photocurrent and fill factor (FF) during the reverse to forward voltage (R-F) scan, (also leading to a reduced open-circuit voltage, $V_{\mathrm{OC}}$ ) as compared to the forward to reverse (F-R) scan. ${ }^{14}$ Inverted hysteresis has been observed as a reduction in the short-circuit current $\left(J_{\mathrm{SC}}\right)$ for F-R scans compared to R-F scans by Shen et al. ${ }^{17}$ and Nemnes et al. ${ }^{18}$ while Tress et al. ${ }^{19}$ observed a loss in the FF in the form of an S-shaped kink for the F-R scan. Both normal and inverted hysteresis trends have been observed to become more acute for faster scans.

Since the observed trends in literature are mostly strong variations in the photocurrent, the general models employed to explain these types of hysteresis were based on variations in the collection efficiency of PSCs, which is modulated by the screening of the internal electric field by the mobile ions. ${ }^{14,20}$ Therefore, normal hysteresis was explained based on the fact that a forward bias voltage corresponded to a favourable charge extraction situation as compared to short-circuit, while this trend is reversed for inverted hysteresis due to a possible extraction barrier at the $\mathrm{TiO}_{2}$ /perovskite interface, where the accumulation of anions at short-circuit creates a dipole that facilitates band alignment and hence efficient charge extraction. ${ }^{19}$

Previously, we have developed a triple-layer architecture of $\mathrm{TiO}_{2} / \mathrm{ZrO}_{2} / \mathrm{Carbon}$ to fabricate a hole-conductor-free printable mesoscopic PSC. ${ }^{21-22}$ By optimizing the mesoporous scaffold and perovskite absorber, hysteresis-less devices have been obtained with $\mathrm{TiO}_{2}$ as the electron transporting layer (ETL). ${ }^{23-24}$ In this study, it was found that the type of hysteresis effect in printable mesoscopic PSCs was

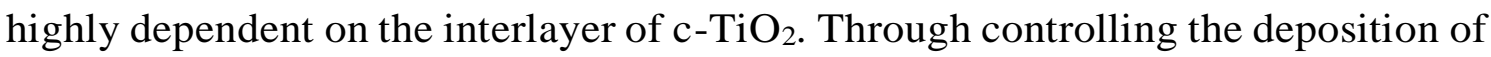
c- $\mathrm{TiO}_{2}$ layer, we realized normal and inverted hysteresis effect for such printable mesoscopic PSCs. Furthermore, the observed hysteresis was uniquely different from the typical hysteresis for PSCs as detailed previously, with the main difference 
in $J$ - $V$ curves between forward and backward scans being the $V_{\mathrm{OC}}$, not $J_{\mathrm{SC}}$ or FF. We analysed the trends of such tunable hysteresis effect by considering the polarization of the $\mathrm{c}-\mathrm{TiO}_{2} /$ perovskite interface, and proposed this phenomenon is associated with the kinetics of accumulation and recombination of ions and charges at this interface during $J$ - $V$ scans.

a

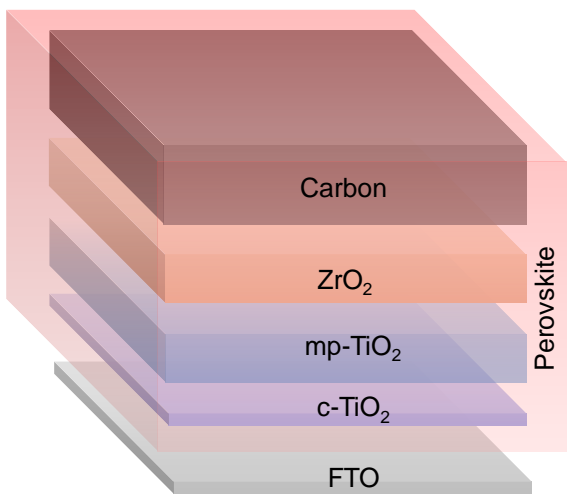

b

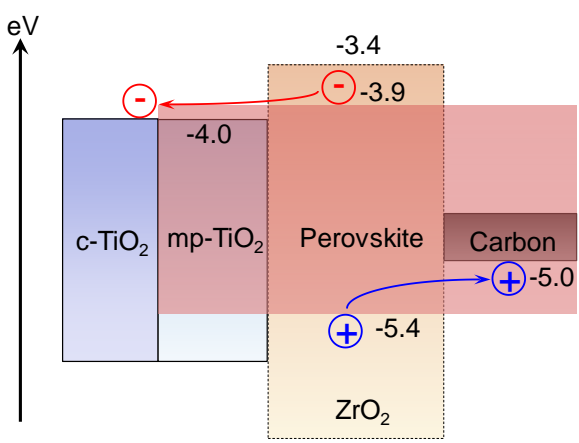

Fig. 1 Device configuration and corresponding working mechanism of a typical printable mesoscopic PSC. (a) Device architecture of $\mathrm{FTO} / \mathrm{c}-\mathrm{TiO}_{2} / \mathrm{mp}-\mathrm{TiO}_{2} / \mathrm{ZrO}_{2}$ /Carbon/Perovskite. The perovskite of (5-AVA)x(MA)1-xPbI3 (5-AVA = 5ammoniumvaleric acid) is infiltrated in the mesoporous layers of $\mathrm{mp}-\mathrm{TiO}_{2}, \mathrm{ZrO}_{2}$ and carbon by simple drop-casting method. (b) Band energy diagram of printable mesoscopic PSCs. The electron-hole pairs generated in the perovskite absorber separates by injecting the electron into $\mathrm{mp}-\mathrm{TiO}_{2}$ and transporting the holes to the carbon electrodes.

At present, there have been many reports of hysteresis-less PSCs that in cases have been obtained by combination of excellent bulk morphologies and manipulation of the contacts, often avoiding the use of metal-oxides. ${ }^{13,25-26}$ In this work, we show that robust devices fabricated with carbon hole extraction electrodes, still show a degree of dynamic hysteresis that is completely controlled by the quality of the thin $\mathrm{TiO}_{2}$ contact layer. We relate the extent of hysteresis observed in different conditions of dynamic scans and waiting times. Remarkably, the transient effects maintain the good features of photocurrent and the shape of $J$ $V$ curves, but affect mainly the apparent photovoltage. The results will be interpreted in terms of the interaction of charge accumulation and recombination effects, following previous observations of transient decays dependence on slow ionic kinetics. ${ }^{27}$ The analysis of photovoltage changes provides a general standpoint for understanding of the operation of contacts in the perovskite based photovoltaics.

\section{Results}




\subsection{Device configuration and working mechanism}

The device architecture used in this study is shown in Fig. 1a, where c-TiO $2, m p-\mathrm{TiO}_{2}$, $\mathrm{ZrO}_{2}$ and carbon layers are deposited on a single FTO glass substrate layer by layer as the scaffold for depositing perovskite absorber. Typically, the three mesoporous layers have a thickness of ca. 1.0, 2.0 and $10.0 \mu \mathrm{m}$, respectively. A mixed-cation perovskite of (5AVA) $x(\mathrm{MA}) 1-\mathrm{xPbI} 3$ (5-AVA $=5$-ammoniumvaleric acid) is infiltrated into the three mesoporous layers as the light absorber, and no HTM layer is needed in the device. The energy band alignment of the device (Fig. 1b) demonstrates that the electron-hole pairs generated in the perovskite absorber separates by injecting the electron into mp- $\mathrm{TiO}_{2}$ and transporting the holes to the carbon back contact. ${ }^{22}$ In this process, the $\mathrm{ZrO}_{2}$ layer effectively prevents the direct contact between $\mathrm{TiO}_{2}$ and carbon layer. The $\mathrm{c}-\mathrm{TiO}_{2}$ layer prevents the valence band holes in the perovskite from reaching the FTO-covered front electrode. With the construction of these selective contacts, the electrons generated in the perovskite absorber are effectively collected by the ETL of the mp- $\mathrm{TiO}_{2}$ and transferred to FTO front electrode via the $\mathrm{c}-\mathrm{TiO}_{2}$, and the holes are collected by the carbon back contact. The mp- $\mathrm{TiO}_{2}$ layer has been considered mainly responsible for the hysteresis in conventional structured PSCs. ${ }^{13,25}$ Particularly, the thickness, pore size and treatment process of the $\mathrm{mp}-\mathrm{TiO}_{2}$ layer have been reported to influence the hysteresis of the devices.28-30 Here, for printable mesoscopic PSCs using a 1- $\mu$ m-thick mp- $\mathrm{TiO}_{2}$ layer, hysteresis-less devices have been fabricated and reported. ${ }^{22}$ We consider the mp- $\mathrm{TiO}_{2}$ scaffold with extremely large surface area is able to provide efficient pathways for electron extraction and transportation rather than cause charge accumulation and possible hysteresis. On the contrary, for the $\mathrm{c}-\mathrm{TiO}_{2}$ layer that blocks hole injection from perovskite into FTO layer, it is critical to achieve a balance between recombination suppression and charge carrier transportation. We have found that the properties of the $\mathrm{c}-\mathrm{TiO}_{2}$ layer and corresponding interfaces significantly affect the $J-V$ hysteresis in the simplified triplelayer architecture based PSCs. For the deposition of c- $\mathrm{TiO}_{2}$ layer, we firstly treated the FTO glass substrates with UV-Ozone for $2.5 \mathrm{~min}$, and then employed an aerosol spray pyrolysis method to prepare a c- $\mathrm{TiO}_{2}$ layer with different spraying cycles (Fig. S1, ESI $\dagger$ ).
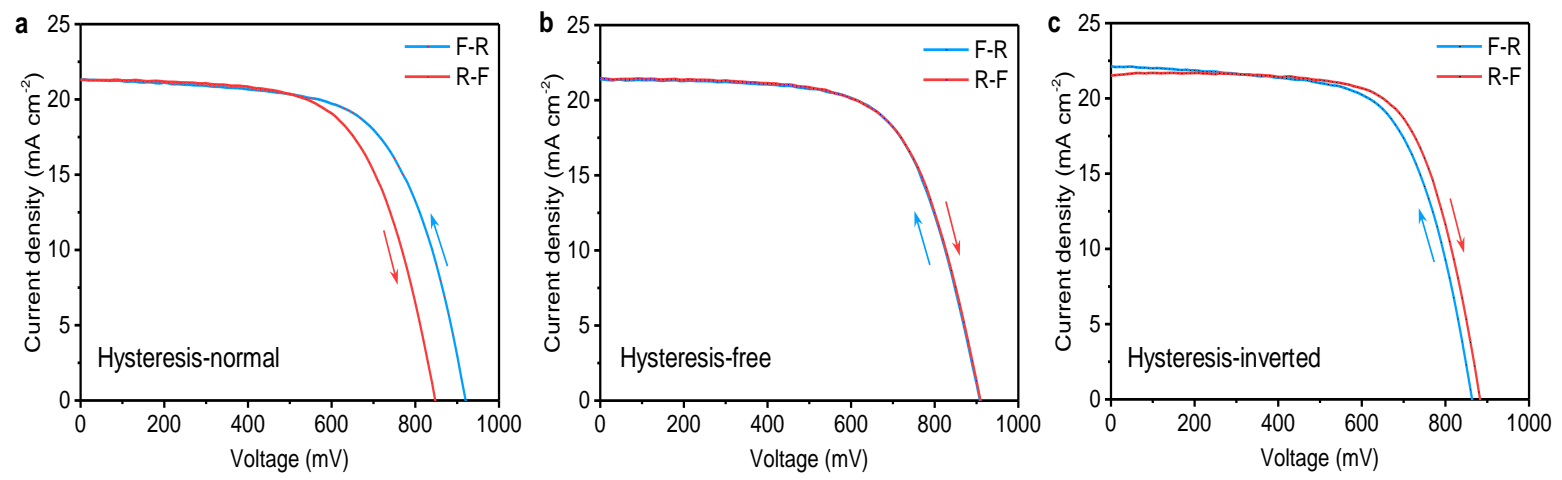

Fig. $2 \mathrm{~J}-V$ curves of printable mesoscopic PSCs with different hysteresis behaviors. (a) Hysteresis-normal device that F-R scan shows better performance than R-F scan; (b) 
Hysteresis-free device that F-R scan and R-F scan show nondistinctive performance; (c) Hysteresis-inverted device that F-R scan shows slightly lower performance than R-F scan. (F-R: from forward bias to reverse bias; R-F: from reverse bias to forward bias; scan rate $\left.250 \mathrm{mVs}^{-1}\right)$.

To evaluate the hysteresis effect of the devices based on a c- $\mathrm{TiO}_{2}$ layer with different spray deposition cycles, $J$ - $V$ curves were measured in forward scan direction (from reverse bias to forward bias, R-F) and backward scan direction (from forward bias to reverse bias, F-R) under simulated AM1.5 $100 \mathrm{~mW} \mathrm{~cm}^{-2}$ sunlight at a scan rate of $250 \mathrm{mV} \mathrm{s}^{-1}$. Note that the scan direction is indicated R-F or F-R, and any waiting time at R or F bias is noted. Before $J-V$ scans, the devices were illuminated for several minutes, which can minimize the influence of the trap states and obtain a steady state. For typical $J-V$ hysteresis phenomenon in PSCs, the photocurrent and efficiency for F-R scan is higher than that for R-F scan. When 4 or 3 spraying cycles of $\mathrm{c}-\mathrm{TiO}_{2}$ layer was applied for printable mesoscopic PSCs, the same trend was observed and we defined this case as hysteresis-normal (Fig. 2a). When we reduced the spraying cycles, it was found that the normal hysteresis effect could be firstly eliminated for 2 cycles (Fig. 2b), and then be inverted (Fig. 2c) completely for 1 cycle, which is regarded as tunable hysteresis effect for printable mesoscopic PSCs. The corresponding devices are coded as hysteresisnormal, hysteresis-free and hysteresis-inverted. Besides the spraying cycles of $\mathrm{c}-\mathrm{TiO}_{2}$ layer, such tunable hysteresis effect is also related to the UV-Ozone treatment (Table S1, ESI $\dagger$ ). It is important to remark that the dynamic hysteresis in our devices mainly affects the apparent photovoltage, and as a consequence the FF, which indicates that recombination changes occurring along the dynamic scan might play a dominant role in the observed changes. Further details on interpretation are presented in the last section of this work.

The photovoltaic parameters of devices showing different hysteresis effects are summarized in Table 1. Generally, the devices fabricated with different cycles sprayed c$\mathrm{TiO}_{2}$ layers show similar performance, especially for the $J_{\mathrm{SC}}$ and PCE. When the spray deposition cycle was reduced from 4 to 1 , the $V_{\mathrm{OC}}$ decreased from $0.92 \mathrm{~V}$ to $0.86 \mathrm{~V}$, and the FF increased from 0.63 to 0.69 . For the parameters measured with F-R and R-F scans, the main differences lie in $V_{\mathrm{OC}}$ rather than $J_{\mathrm{SC}}$ or $\mathrm{FF}$. This is quite different from the trend of typical hysteresis phenomenon for PSCs. ${ }^{12}$ 

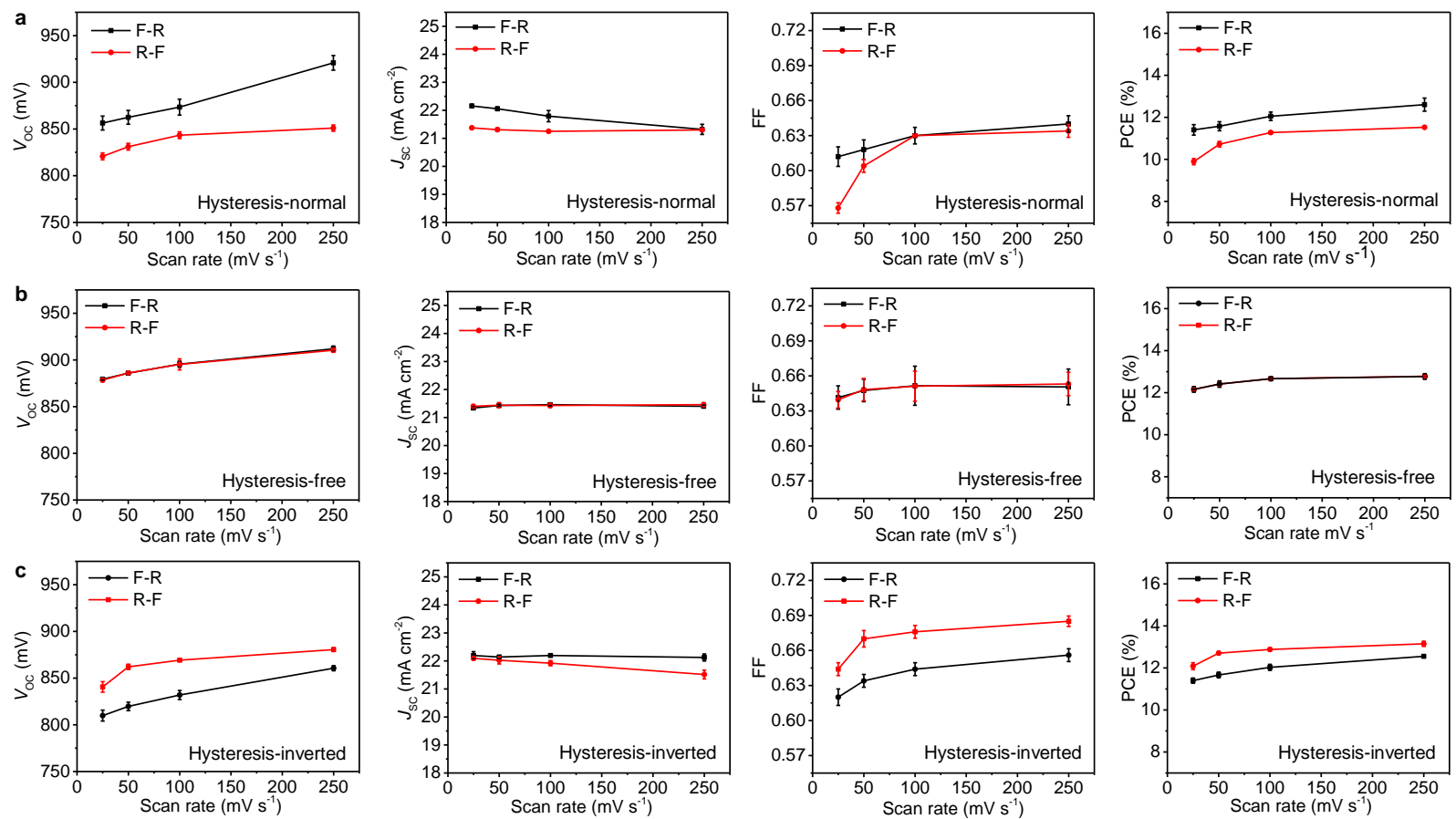

Fig. 3 Dependence of photovoltaic parameters on scan directions and scan rates. (a) hysteresis-normal, (b) hysteresis-free and (c) hysteresis-inverted printable mesoscopic PSCs.

Besides scan directions, the scan rates also influence the hysteresis behaviors of PSCs. To investigate the rate-dependent hysteresis effect of the devices, the photovoltaic parameters were measured at a scan rate of $25 \mathrm{mV} \mathrm{s}^{-1}, 50 \mathrm{mV} \mathrm{s}^{-1}, 100 \mathrm{mV} \mathrm{s}^{-1}$ and $250 \mathrm{mV}$ $\mathrm{s}^{-1}$, and compared in Fig. 3. Although the parameters measured at different scan rates varied, the differences between the values obtained with F-R and R-F scans still provide essential information for the hysteresis behaviors of printable mesoscopic PSCs. For hysteresis-normal devices (Fig. 3a), the difference in $V_{\mathrm{OC}}$ for F-R and R-F scans reduced from $\sim 70 \mathrm{mV}$ to $\sim 20 \mathrm{mV}$ when the scan rate decreased from $250 \mathrm{mV} \mathrm{s}^{-1}$ to $25 \mathrm{mV} \mathrm{s}^{-1}$, while the difference in $J_{\mathrm{SC}}$ increased slightly. For the overall PCE, F-R at all scan rates led to a higher value than R-F. For hysteresis-free devices (Fig. 3b), F-R and R-F scans showed almost the same photovoltaic parameters of $V_{\mathrm{OC}}, J_{\mathrm{SC}}, \mathrm{FF}$ and PCE at all different scan rates, though the values decreased slightly when the scan slowed down. For hysteresis-inverted devices (Fig. 3c), R-F scan resulted in higher $V_{\mathrm{OC}}$ and FF while lower $J_{\mathrm{SC}}$ than F-R scan, leading to higher PCE, and the difference between R-F and F-R scans did not change much for all the scan rates. 


\begin{tabular}{|c|c|c|c|c|c|c|c|}
\hline Hysteresis & $\mathrm{c}-\mathrm{TiO}_{2}$ & Scan direction & $V_{O C}(\mathrm{~V})$ & $\left.J_{\mathrm{SC}}(\mathrm{mA} \mathrm{cm})^{-2}\right)$ & $\mathrm{FF}$ & PCE & HEI \\
\hline \multirow{2}{*}{ Normal } & \multirow{2}{*}{4 cycle } & F-R & 0.92 & 21.32 & 0.64 & 12.60 & \multirow{2}{*}{$0.0422 \pm 0.0068$} \\
\hline & & $\mathrm{R}-\mathrm{F}$ & 0.85 & 21.30 & 0.63 & 11.52 & \\
\hline \multirow{2}{*}{ Normal } & \multirow{2}{*}{3 cycle } & F-R & 0.92 & 21.40 & 0.63 & 12.37 & \multirow{2}{*}{$0.0171 \pm 0.0054$} \\
\hline & & $\mathrm{R}-\mathrm{F}$ & 0.89 & 21.33 & 0.63 & 11.87 & \\
\hline \multirow{2}{*}{ Free } & \multirow{2}{*}{2 cycle } & F-R & 0.91 & 21.40 & 0.65 & 12.77 & \multirow{2}{*}{$0.0010 \pm 0.0035$} \\
\hline & & R-F & 0.91 & 21.45 & 0.65 & 12.78 & \\
\hline \multirow{2}{*}{ Inverted } & \multirow{2}{*}{1 cycle } & F-R & 0.86 & 22.14 & 0.66 & 12.55 & \multirow{2}{*}{$-0.0434 \pm 0.0093$} \\
\hline & & R-F & 0.88 & 21.51 & 0.69 & 13.15 & \\
\hline
\end{tabular}

Table 1 Hysteresis effect, photovoltaic parameters and hysteresis effect index (HEI) of the devices fabricated using $\mathrm{c}-\mathrm{TiO}_{2}$ layer with different spray deposition cycles.

Series resistance $\left(R_{\mathrm{S}}\right)$ and shunt resistance $\left(R_{\mathrm{SH}}\right)$ of devices with different hysteresis behaviors are also summarized in Table S2 (ESI $\dagger$ ) and compared in Figure S2 (ESI $\dagger$ ). All $R_{\mathrm{S}}$ slightly decreased as the scan rate increased from 50 to $250 \mathrm{mV} \mathrm{s}^{-1}$, while $R_{\mathrm{SH}}$ shows much more stable values as scan rates increased. Particularly, hysteresis-free devices showed slightly larger $R_{\mathrm{S}}$, but similar $R_{\mathrm{SH}}$, compared with hysteresis-normal and hysteresis-inverted devices independently of the scan rates. For $R_{\mathrm{S}}$ and $R_{\mathrm{SH}}$ measured at F-R and R-F scans, hysteresis-normal and hysteresis-inverted devices showed similar $R_{\mathrm{S}}$, but larger $R_{\mathrm{SH}}$ at F-R scans. Hysteresis-free devices showed similar $R_{\mathrm{SH}}$, but smaller $R_{\mathrm{S}}$ at F-R scans.
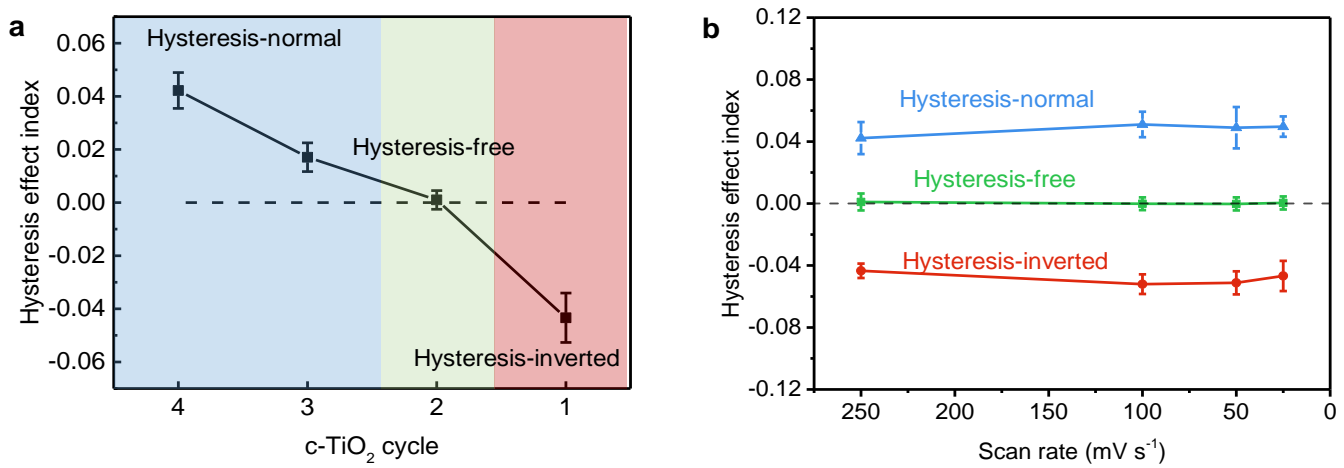

Fig. 4 Dependence of hysteresis effect index (HEI) (a) on c- $\mathrm{TiO}_{2}$ layer spray deposition cycles and (b) on scanning rates for hysteresis-normal, hysteresis-free and hysteresisinverted devices. 


\subsection{Hysteresis index characterization}

A hysteresis index has been defined to describe the differences in $J-V$ curves appearing near open-circuit condition. ${ }^{28}$ In our case, since the hysteresis has been inverted causing the R-F scan to show higher current density and overall performance, a modified $J-V$ hysteresis effect index (HEI) is defined by Equation 1.

$$
\text { Hysteresis effect index }(\mathrm{HEI})=\frac{J_{R S(0.8 \mathrm{Voc})}-J_{F S(0.8 V o c)}}{\left(J_{R S\left(0.8 V_{o c}\right)}+J_{F S(0.8 V o c)}\right) / 2}
$$

where $J_{\mathrm{RS}(0.8 \mathrm{Voc})}$ and $J_{\mathrm{FS}(0.8 \mathrm{Voc})}$ represent current-density at $80 \%$ of $V_{\mathrm{OC}}$ for F-R and R-F scans, respectively. Even though our observations differ markedly for the early observations that lead to the definition of HEI in terms of current difference, ${ }^{15}$ the HEI shown in Fig. 4 still serves to illustrate the consistent evolution of $J$ - $V$ curves depending on the changes of the $\mathrm{TiO}_{2}$ compact layer. For hysteresis-normal devices, the $J_{\mathrm{RS}(0.8 V O C)}$ is

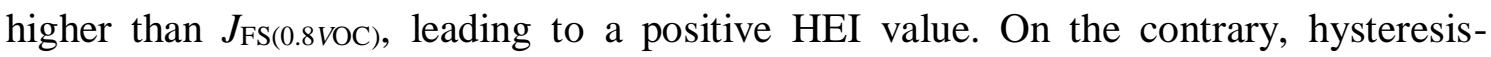
inverted devices show negative HEI values, as shown in Table 1. The reduction of $\mathrm{c}-\mathrm{TiO}_{2}$ spray deposition cycles resulted in significant decrease of HEI from positive values of 0.0422 and 0.017 to 0.001 , then to a negative value of -0.0434 (Fig. 4a). The transition of HEI from hysteresis-normal to hysteresis-inverted is almost linear to the spray deposition cycles of $\mathrm{c}-\mathrm{TiO}_{2}$. Due to the relatively stable $J_{\mathrm{SC}}$ at different scan rates, the HEI values of all the three types of devices also stayed almost unchanged as the scan rate decreased (Fig. 4b). Considering that such printable mesoscopic PSCs show different trends for hysteresis effect, the variations in $V_{\mathrm{OC}}$ provide more key information for analysing such tunable hysteresis for printable mesoscopic PSCs.
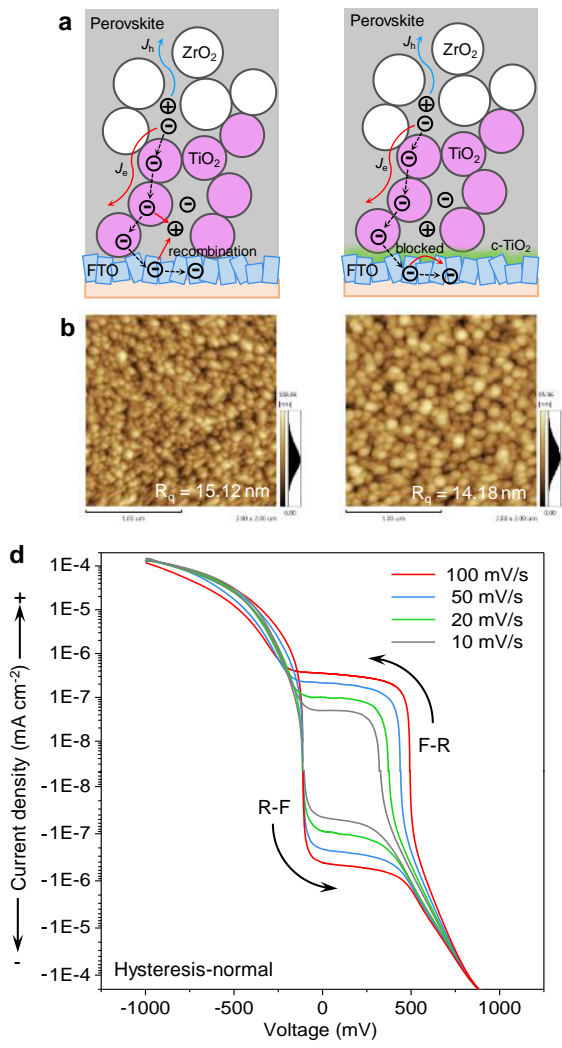
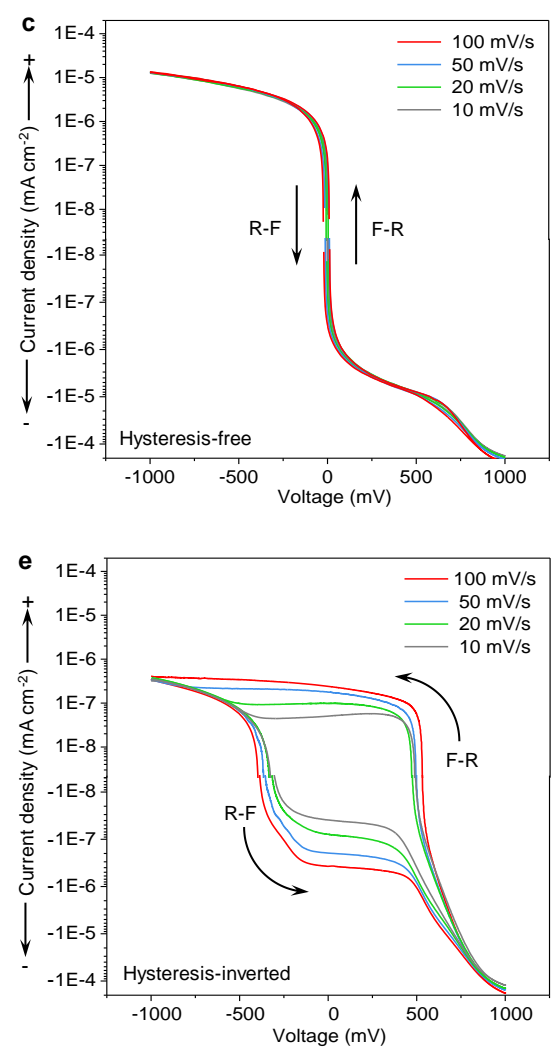
Fig. 5 Functions of $\mathrm{c}-\mathrm{TiO}_{2}$ layer in printable mesoscopic PSCs and capacitance analysis of the devices. (a) Electron and hole separation/transportation in FTO/mp$\mathrm{TiO}_{2} / \mathrm{ZrO}_{2} /$ perovskite, and $\mathrm{FTO} / \mathrm{c}-\mathrm{TiO}_{2} / \mathrm{mp}-\mathrm{TiO}_{2} / \mathrm{ZrO}_{2} /$ perovskite. (b) AFM images of bare FTO substrate and $\mathrm{c}-\mathrm{TiO}_{2}$ sprayed FTO substrate. (c-e) dark $J-V$ curves at room temperature in logarithm scaled current representation at different scan rates for hysteresis-free, hysteresis-normal and hysteresis-inverted devices. The arrows in vertical scale indicate the direction of increasing current, either positive or negative.

\subsection{Characterization of $\mathrm{c}-\mathrm{TiO}_{2}$ layer}

Ion migration with the perovskite film, high trap-state density for charge carriers at the perovskite surface and unbalanced charge carrier transport have been considered as the origins of the hysteresis effect for PSCs. ${ }^{3,27,28}$ Great efforts have been made to develop deposition methods that are able to obtain high-quality perovskite crystals. Typically, perovskite layers with large grain size, uniform morphology and appropriate composition will possess low defect density and show restricted ion migrations, which enable hysteresis-free/less devices with various architectures. ${ }^{25,31-32}$ In our case, the formation of the perovskite crystals was firstly templated by 5-AVA cations with preferential growth in the normal direction, and then hosted by the scaffold of $\mathrm{TiO}_{2} / \mathrm{ZrO}_{2} /$ Carbon triple-layer. Thus, the hysteresis effect originating from the quality of perovskite crystals has been minimized. Considering the remarkable device stability under working conditions, we propose that the $J$ - $V$ hysteresis effect in our devices is mainly due to the charge accumulation at the interfaces rather than composition change in the perovskite crystals, which would cause severe performance degradation of the devices. ${ }^{33-34}$ In printable mesoscopic PSCs, the mp- $\mathrm{TiO}_{2}$ collects and transfer the electrons as the ETL, while c$\mathrm{TiO}_{2}$ layer works as a selective contact layer that transports electrons but blocks holes. Without a c- $\mathrm{TiO}_{2}$ layer, the perovskite absorber will directly contact FTO front electrode, resulting in significant charge recombination and voltage loss for the device (Fig. 5a). Unfortunately, the electron mobility of $\mathrm{TiO}_{2}$ is only about $10^{-4} \mathrm{~cm}^{2} \mathrm{~V}^{-1} \mathrm{~s}^{-1}, 35-36$ which is much lower than the transportation of charge carriers in $\mathrm{MAPbI}_{3}\left(10^{-2}-10^{0} \mathrm{~cm}^{2} \mathrm{~V}^{-1} \mathrm{~s}^{-1}\right) .{ }^{37}$ Thus, it was proposed that the $\mathrm{mp}-\mathrm{TiO}_{2}$ layer in conventional structured PSCs would limit the electron transport, and cause capacitive hysteresis. ${ }^{38-39}$ Herein we show that the hysteresis in c- $\mathrm{TiO}_{2}$ and $\mathrm{mp}-\mathrm{TiO}_{2}$ based PSCs is mainly dependent on the $\mathrm{c}-\mathrm{TiO}_{2}$ layer and associated interfaces, not on the $\mathrm{mp}-\mathrm{TiO}_{2}$ layer. As we show later, the $\mathrm{c}-\mathrm{TiO}_{2}$ surface meets the perovskite layer and here the ionic and electronic carriers find a barrier and establish essential charge accumulation and bandbending that when combined with surface recombination rates play a key role in the apparent voltage in transient conditions. In addition, the accumulation of electrons at the thin $\mathrm{c}-\mathrm{TiO}_{2}$ itself is also an important factor to control the measured voltage. These elements will form the main considerations in our model, which motivates further analysis of the structure of the $\mathrm{c}-\mathrm{TiO}_{2}$ layer.

It should be noted that the deposition of $\mathrm{c}-\mathrm{TiO}_{2}$ layer between mp- $\mathrm{TiO}_{2}$ and $\mathrm{FTO}$ layers also improve the contact and facilitate the charge transfer at the interfaces. Atomic force 
microscope (AFM) and scanning electron microscope (SEM) measurements were performed to unravel the differences of the surface morphology of bare and $\mathrm{c}-\mathrm{TiO}_{2}$ sprayed FTO substrates (Fig. 5b and Fig. S3†). The FTO substrate possessed a polyhedron composed morphology with a root-mean-square (RMS) roughness of $15.12 \mathrm{~nm}$. After spraying a $\mathrm{c}-\mathrm{TiO}_{2}$ layer, the surface turned to be composed of sphere-shape particles and the roughness decreased slightly to $14.18 \mathrm{~nm}$. Due to the rough surface of FTO layer, we cannot accurately obtain the thickness of the $\mathrm{c}-\mathrm{TiO}_{2}$ layer sprayed on FTO substrates with 1 to 4 cycles. Instead, we sprayed the $\mathrm{c}-\mathrm{TiO}_{2}$ layer on a silicon wafer with 8 cycles, and measured its thickness by a profilometer. The thickness of the $\mathrm{c}-\mathrm{TiO}_{2}$ layer was $52.7 \pm 5.50$ $\mathrm{nm}$. Thus, we suppose the thickness of $\mathrm{c}-\mathrm{TiO}_{2}$ layers sprayed with $1-4$ cycles was $6.59 \pm 0.69 \mathrm{~nm}, 13.18 \pm 1.37 \mathrm{~nm}, 19.76 \pm 2.06 \mathrm{~nm}$ and $26.35 \pm 2.75 \mathrm{~nm}$, respectively. It is expected that the FTO particles are wrapped by an ultra-thin $\mathrm{TiO}_{2}$ film, and this can be regarded as an interface modification rather than formation of an extra interlayer.
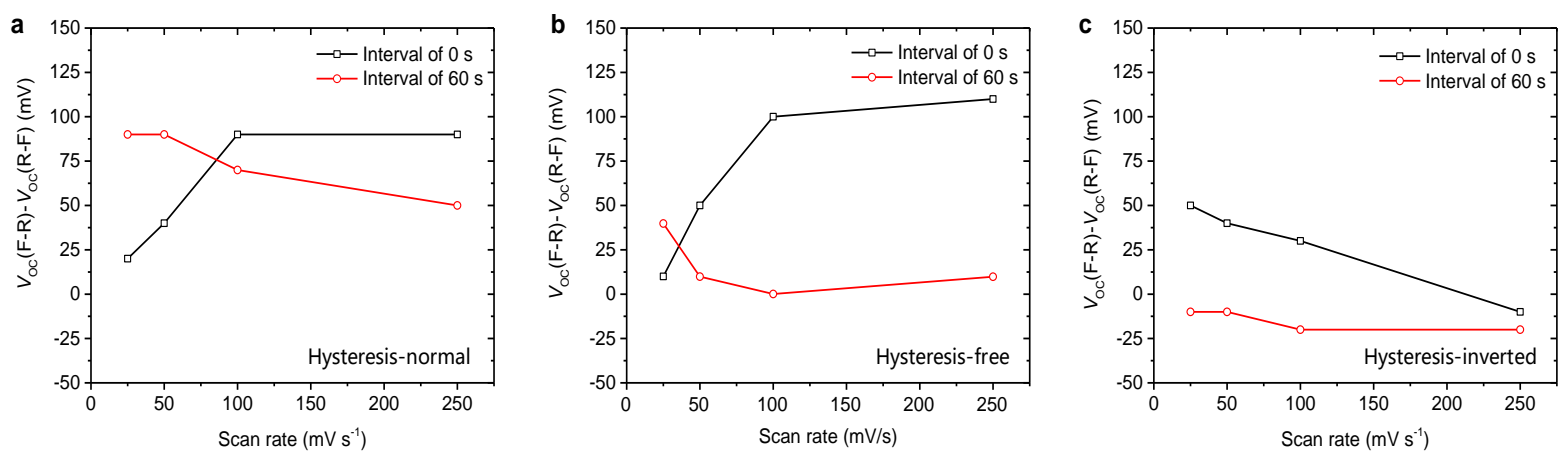

Fig. 6 Dependence of $\left[\mathrm{V}_{\mathrm{OC}}(\mathrm{F}-\mathrm{R})-\mathrm{V}_{\mathrm{OC}}(\mathrm{R}-\mathrm{F})\right]$ on scan rates. The hysteresis (a) -normal, (b) -free and (c) -inverted devices were continuously scanned with an interval of $0 \mathrm{~s}$ or discontinuously scanned with an interval of $60 \mathrm{~s}$ at $250 \mathrm{mV} \mathrm{s}-1,100 \mathrm{mV} \mathrm{s}-1,50 \mathrm{mV} \mathrm{s}-1$ and $25 \mathrm{mV}$ s-1 firstly in F-R direction, and then in R-F direction. The $\mathrm{V}_{\mathrm{OC}}$ values were summarized and compared as $\left[\mathrm{V}_{\mathrm{OC}}(\mathrm{F}-\mathrm{R})-\mathrm{V}_{\mathrm{OC}}(\mathrm{R}-\mathrm{F})\right]$ versus scan rates.

XPS measurements were carried out to investigate the Ti element in the 1-4 cycle c$\mathrm{TiO}_{2}$ sprayed FTO substrates. The Ti peak areas were summarized and compared using Sn peak as a reference peak. The Ti/Sn ratio increased significantly from 1.581 to 3.93 , then to 10.3 and 22.1 for $1-4$ cycle c-TiO 2 spraying (Fig. S4, ESI $\uparrow$ ). In addition, the work function of the $\mathrm{c}-\mathrm{TiO}_{2}$ sprayed FTO substrates were characterized, which decreased slightly from $4.79 \mathrm{eV}$ for the bare FTO to $4.45-4.62 \mathrm{eV}$ for the 1-4 cycle $\mathrm{c}-\mathrm{TiO}_{2}$ sprayed FTO (Fig. S5, ESI $\dagger$ ). The influence of the $\mathrm{c}^{-\mathrm{TiO}_{2}}$ layer on the charge extraction was investigated by time-resolved photoluminescence (TRPL) spectra (Fig. S6, ESI $\uparrow$ ). Semidevices of $\mathrm{FTO} / \mathrm{c}-\mathrm{TiO}_{2} / \mathrm{mp}-\mathrm{TiO}_{2} / \mathrm{ZrO}_{2} /$ Perovskite were fabricated with 1-4 cycles sprayed c- $\mathrm{TiO}_{2}$ layer. A very fast decay followed by a slower decay was observed for all samples, as also observed elsewhere. ${ }^{40}$ The curves were fitted by a biexponential decay function, and the results are summarized in Table S3. It was found that $\tau_{1}$ and $\tau_{2}$ are almost identical as the $\mathrm{c}-\mathrm{TiO}_{2}$ spraying cycles increased, indicating thicker $\mathrm{c}-\mathrm{TiO}_{2}$ layer does not 
significantly modify the charge extraction of the c- $\mathrm{TiO}_{2} / \mathrm{mp}-\mathrm{TiO}_{2}$ bilayer.

\subsection{Capacitive hysteresis characterization}

Capacitance analysis of voltage sweep under dark was performed to investigate the capacitive hysteresis effect in printable mesoscopic PSCs (Fig. 5c-e), which was proposed to be caused by the charge accumulation at the $\mathrm{TiO}_{2} /$ perovskite interface. ${ }^{38-39}$ The voltage variation, $\Delta V$, applied to the solar cell produces a transient response before reaching the steady state. The time interval $\Delta t$ between applied voltage steps might be shorter than the time of relaxation. In the cases in which charging process occurs, an additional transient current is expected to be observed caused by the time variation of the accumulated charges. Furthermore, capacitive currents should vanish as the scan rate approaches steady-state conditions. For the hysteresis-normal device (Fig. 5d), significant capacitive hysteresis was observed along with similar dark current density with hysteresis-free and hysteresis-inverted devices at a forward (positive) bias. When spraying cycles for c- $\mathrm{TiO}_{2}$ layer decreased from 4 to 1, the capacitive hysteresis effect firstly vanished (Fig. 5c), and then came back (Fig. 5e). The dark capacitive trends indicate a strong polarization may exist at the $\mathrm{c}-\mathrm{TiO}_{2} /$ perovskite interface, ${ }^{38}$ which can have a significant impact on the hysteretic trends under illumination.

\subsection{Effect of delay time}

As mentioned in the previous section, for the hysteresis effect of printable mesoscopic PSCs, the main difference between F-R and R-F scans lies in $V_{\mathrm{OC}}$ rather than $J_{\mathrm{SC}}$ or FF. Thus, we summarized and compared the $V_{\mathrm{OC}}$ for F-R and R-F scans, presented as [ $V_{\mathrm{OC}}(\mathrm{F}-$ $\left.\mathrm{R})-V_{\mathrm{OC}}(\mathrm{R}-\mathrm{F})\right]$ versus scan rates in Fig. 6. Notably, we found the intervals and sequence between $J$ - $V$ scans also influence the device performance, which can provide us with some key information. In this case the $V_{\mathrm{OC}}$ was measured by sequential scans with interval of 0 $\mathrm{s}$ or $60 \mathrm{~s}$ at $250 \mathrm{mV} \mathrm{s}^{-1}, 100 \mathrm{mV} \mathrm{s}^{-1}, 50 \mathrm{mV} \mathrm{s}^{-1}$ and $25 \mathrm{mV} \mathrm{s}^{-1}$ firstly in F-R direction, and then in R-F direction. The dependence of $V_{\mathrm{OC}}$ on scan rates is shown in Fig. S7 (ESI†).

Without any delay (interval of $0 \mathrm{~s}$ ) between scans, $\left[V_{\mathrm{OC}}(\mathrm{F}-\mathrm{R})-V_{\mathrm{OC}}(\mathrm{R}-\mathrm{F})\right]$ decreased as the scan rate slowed down from $250 \mathrm{mV} \mathrm{s}^{-1}$ to $25 \mathrm{mV} \mathrm{s}^{-1}$ for hysteresis-normal and hysteresis-free devices, while increased for the hysteresis-inverted device. Remarkably, in this condition, the hysteresis-inverted device showed higher $V_{\mathrm{OC}}$ and PCE for F-R scans. In other words, this hysteresis-inverted device showed normal hysteresis effect. When an interval of $60 \mathrm{~s}$ was applied between the scans, the evolution of the $V_{\mathrm{OC}}$ changed significantly. For hysteresis-normal and hysteresis-free devices, [ $\left.V_{\mathrm{OC}}(\mathrm{F}-\mathrm{R})-V_{\mathrm{OC}}(\mathrm{R}-\mathrm{F})\right]$ increased as the scan rate slowed down, which showed completely opposite trends against the results obtained with interval of $0 \mathrm{~s}$. Significantly, the values of $\left[V_{\mathrm{OC}}(\mathrm{F}-\mathrm{R})-V_{\mathrm{OC}}(\mathrm{R}-\mathrm{F})\right]$ for hysteresis-inverted device became negative, and was consistent with the results discussed in the previous section.

\section{Discussion}


(a)

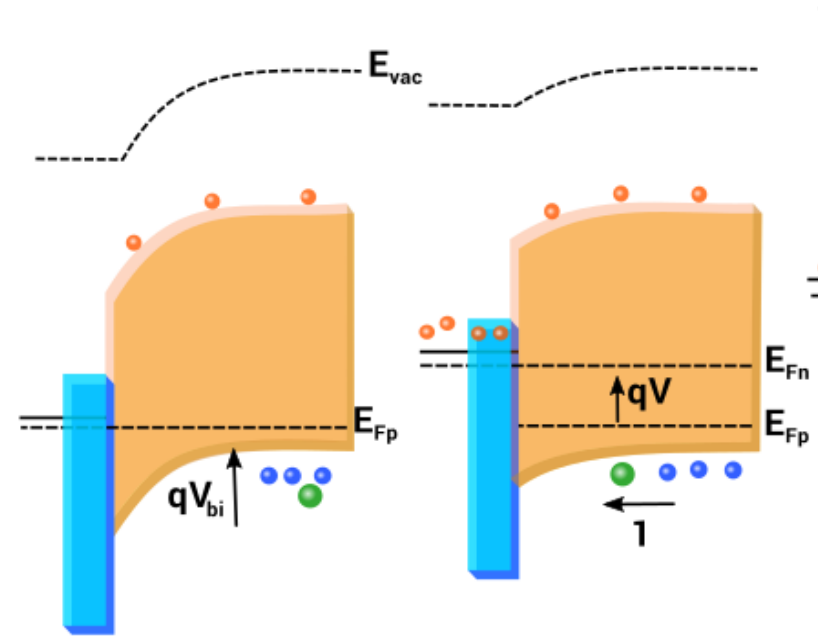

(c)

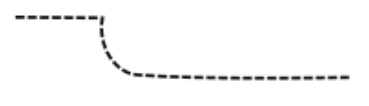

2

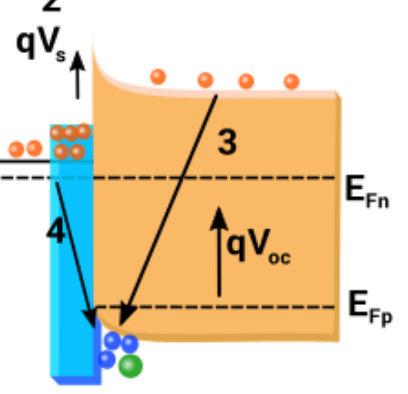

Fig. 7 Schematic diagram of the $\mathrm{c}-\mathrm{TiO}_{2}$ (blue layer)/perovskite (orange film) interface indicating the processes governing the $\mathrm{V}_{\mathrm{OC}}$. We consider a p-type perovskite at short circuit condition shown in (a) with a built-in potential at the perovskite/c- $\mathrm{TiO}_{2}$ interface (electrons represented in red, holes and cations in blue and green respectively). (b) The PSC under an applied forward bias. Process 1 indicates the kinetics of drift of cations and holes towards the interface. (c) The PSC at large forward bias ( $\mathrm{V}_{\text {OC }}$ ). The accumulation of cations and holes at the interface creates an upward band bending which can be described by a surface voltage $V_{s}$ represented in 2 . These accumulated charges can act as a preferential zone for both recombination with electrons in the bulk (3) and in the c$\mathrm{TiO}_{2} /$ FTO region. Recombination pathway 4 crucially dependent on the thickness of the c- $-\mathrm{TiO}_{2}$ layer, and is the dominant mechanism controlling recombination rates of accumulated charges and the output $\mathrm{V}_{\mathrm{OC}}$ in a transient scan.

Since the $V_{\mathrm{OC}}$ is the main photovoltaic variable modified in the type of hysteresis in our cells, the mechanisms of hysteresis cannot be explained based on the models of charge collection presently dominant in the literature. The observation of a consistent $J_{\mathrm{SC}}$ irrespective of scan rate and direction indicates that charge transport and extraction is identical at low $\mathrm{F}$ biases, pointing to a preferential recombination mechanism at large $\mathrm{F}$ biases, modifying the output voltage. In consideration of the above arguments, we explain the trends observed by considering the dominant contribution of the $\mathrm{c}-\mathrm{TiO}_{2} /$ perovskite interface. Fig. 7 provides a picture of the charge accumulation and polarization features at the interface. The formation of an accumulation of cations and electronic holes at the c- $-\mathrm{TiO}_{2} /$ perovskite interface under illumination has been discussed ${ }^{41}$ and is shown in Fig. 7c. Note that the morphology of the mesoporous layer is neglected as it is assumed that rather narrow band bending occurs at the perovskite side. This accumulation of cations and electronic holes in a stabilized situation creates excess potential at the c$\mathrm{TiO}_{2} /$ perovskite interface that enhances $V_{\mathrm{OC}}$. It also serves as a strong source of surface 
recombination, by electrons coming from the $\mathrm{TiO}_{2}$ side of the contact.

Slow kinetic features result in hysteresis patterns that are mainly induced by the sluggish ionic dynamics, as already remarked on the capacitive response in Fig. 5. The ionic displacement is completed at F voltage at the start of an F-R scan, causing the large accumulation, Fig. 7c, which is almost a steady-state value that is not modulated by ionic drift rates. Contrarily, for the R-F scan, the accumulation is delayed, which creates an asymmetry of $V_{\mathrm{OC}}$ values. In an R-F scan, the ions are moved away at $\mathrm{R}$ bias, so that the voltage cannot reach the same value as that of the F-R scan, explaining the normal hysteresis. It is noted from Fig. 2 that the F-R $V_{\mathrm{OC}}$ is identical for normal hysteresis and hysteresis-free devices but is reduced by $\sim 50 \mathrm{mV}$ for the inverted hysteresis samples. This indicates that another major factor to consider in the variation of photovoltage is the amount of recombination, as commented before. For thinner c- $\mathrm{TiO}_{2}$ layer, the covering of FTO may be incomplete, facilitating recombination of surface electrons as indicated in Fig. 7c. (process 4). The R-F scan $V_{\mathrm{OC}}$ is increased by $\sim 70 \mathrm{mV}$ for the hysteresis-free and inverted hysteresis devices. This is also attributed to the larger work function of the thin c- $\mathrm{TiO}_{2}$ layers (Fig. S5, ESI $\uparrow$ ), creating smaller built-in potential for the perovskite, which allows for larger accumulation at a given $F$ voltage in the R-F scan. For the inverted hysteresis devices, the large R-F voltage is sustained likely due to the sluggish kinetics of the surface recombination indicated in (4) in Fig. 7c. The improved R-F $V_{\mathrm{OC}}$ values contribute to the hysteresis-free behavior, and in conjunction with the $V_{\mathrm{OC}}$ drop in the F$\mathrm{R}$ direction for a very thin $\mathrm{c}-\mathrm{TiO}_{2}$ layer, yields the inverted hysteresis trend in our samples. TRPL analysis showed that the kinetics of charge extraction is rather similar in the different thickness cases so that differences of interfacial kinetics does not appear a significant factor controlling the changes of hysteresis pattern.

We now proceed to explain the rich variety of behaviors of the dependence of $V_{\mathrm{OC}}$ on the scan rates (Fig. 3) and waiting times (Fig. S7, ESI $\dagger$ ). For the F-R scan, the accumulated positive charges at the surface at steady state recombine immediately upon cycling the voltage. Therefore, faster scans provide a larger $V_{\mathrm{OC}}$ in the F-R direction. This is observed for all devices, irrespective of the type of hysteresis, Fig. 3. The effect of waiting time under illumination is identical, where the accumulation is enhanced due to the photogenerated carriers, providing larger output voltages in all devices except the hysteresis-inverted devices. This could occur due to the increased surface recombination of photogenerated carriers for very thin $\mathrm{c}-\mathrm{TiO}_{2}$ layers previously described. For the $\mathrm{R}-\mathrm{F}$ scans, the $V_{\mathrm{OC}}$ improves for faster scan rates since the positive ions do not have enough time to drift away from the $\mathrm{c}-\mathrm{TiO}_{2}$ layer, thereby improving the $V_{\mathrm{OC}}$. The effect of waiting time under illumination is for the photovoltage to drive positive ions towards the $\mathrm{c}^{-\mathrm{TiO}_{2}}$, thereby facilitating accumulation and also enhanced $V_{\mathrm{OC}}$ during the RF scan.

In summary, we observe that the interplay of slow dynamics of charge accumulation and changes of recombination rates explains the observed hysteresis patterns. Hysteresis in PSCs is regarded as a combination of processes inherent to the operation of this device and not a single effect that can be removed. However, high quality of materials and 
interface do minimize the influence of dynamic hysteresis, which mainly affects the photovoltage according to the scanning procedure.

\section{Conclusions}

We have identified a tunable hysteresis effect consisting of $V_{\mathrm{OC}}$ variations where the type of hysteresis can be altered solely through modification of the $\mathrm{c}-\mathrm{TiO}_{2}$ layer. The trends of tunable hysteresis effect are considered here to be caused by the polarization of the $\mathrm{c}-\mathrm{TiO}_{2} /$ perovskite interface, and is related to the kinetics of accumulation of positive charges at this interface during $J-V$ scans, in combination with the extent of surface recombination. The nature and properties of the $\mathrm{c}-\mathrm{TiO}_{2} /$ perovskite interface are critical for the recombination rates of accumulated charges at this interface, which is the dominant mechanism controlling the hysteretic trends in the PSCs. The demonstration of such tunable hysteresis effect in printable mesoscopic PSCs shows that the properties of c$\mathrm{TiO}_{2} /$ perovskite interface are crucial for controlling the device performance metrics. We showed that hysteresis-free devices can be formed by adapting specific conditions of the contact from a broad range of possibilities, and that hysteresis-free devices may not coincide with optimal solar cell operation. Therefore, we suggest that understanding the phenomenon of hysteresis is necessary for a better control of PSCs materials and interfaces.

\section{Acknowledgements}

The authors acknowledge financial support from the National Natural Science Foundation of China (91433203, 61474049, 51502141, 21702069), the Ministry of Science and Technology of China (2015AA034601), the 111 Project (No. B07038), and the China Postdoctoral Science Foundation (2016M600588). We also acknowledge funding from MINECO of Spain under Project MAT2016-76892-C3-1-R and Generalitat Valenciana Project PROMETEOII/2014/020. S. R. acknowledges Generalitat Valenciana for the grant (GRISOLIA/2014/034).

\section{Notes and references}

1. Kojima, A.; Teshima, K.; Shirai, Y.; Miyasaka, T. Organometal Halide Perovskites as Visible-Light Sensitizers for Photovoltaic Cells. Journal of the American Chemical Society 2009, 131 (17), 6050-6051.

2. Kim, H.-S.; Lee, C.-R.; Im, J.-H.; Lee, K.-B.; Moehl, T.; Marchioro, A.; Moon, S.-J.; Humphry-Baker, R.; Yum, J.-H.; Moser, J. E.; Grätzel, M.; Park, N.-G. Lead iodide perovskite sensitized all-solid-state submicron thin film mesoscopic solar cell with efficiency exceeding 9\%. Scentific Reports 2012, 2, 591.

3. Green, M. A.; Ho-Baillie, A.; Snaith, H. J. The emergence of perovskite solar cells. Nature Photonics 2014, 8 (7), 506-514. 
4. Im, J.-H.; Lee, C.-R.; Lee, J.-W.; Park, S.-W.; Park, N.-G. $6.5 \%$ efficient perovskite quantum-dot-sensitized solar cell. Nanoscale 2011, 3 (10), 4088-4093.

5. Xing, G.; Mathews, N.; Sun, S.; Lim, S. S.; Lam, Y. M.; Grätzel, M.; Mhaisalkar, S.; Sum, T. C. Long-range balanced electron-and hole-transport lengths in organicinorganic $\mathrm{CH}_{3} \mathrm{NH}_{3} \mathrm{PbI}_{3}$. Science 2013, 342 (6156), 344-347.

6. Dong, Q.; Fang, Y.; Shao, Y.; Mulligan, P.; Qiu, J.; Cao, L.; Huang, J. Electronhole diffusion lengths $>175 \mu \mathrm{m}$ in solution-grown $\mathrm{CH}_{3} \mathrm{NH}_{3} \mathrm{PbI}_{3}$ single crystals. Science 2015, 347 (6225), 967-970.

7. Yang, W. S.; Park, B.-W.; Jung, E. H.; Jeon, N. J.; Kim, Y. C.; Lee, D. U.; Shin, S. S.; Seo, J.; Kim, E. K.; Noh, J. H.; Seok, S. I. Iodide management in formamidiniumlead-halide-based perovskite layers for efficient solar cells. Science 2017, 356 (6345), 1376.

8. Eames, C.; Frost, J. M.; Barnes, P. R. F.; O'Regan, B. C.; Walsh, A.; Islam, M. S. Ionic transport in hybrid lead iodide perovskite solar cells. Nature Communications 2015, 6, 7497.

9. Kim, H.-S.; Mora-Sero, I.; Gonzalez-Pedro, V.; Fabregat-Santiago, F.; JuarezPerez, E. J.; Park, N.-G.; Bisquert, J. Mechanism of carrier accumulation in perovskite thin-absorber solar cells. Nature Communications 2013, 4, 2242.

10. Bertoluzzi, L.; Sanchez, R. S.; Liu, L.; Lee, J.-W.; Mas-Marza, E.; Han, H.; Park, N.-G.; Mora-Sero, I.; Bisquert, J. Cooperative kinetics of depolarization in $\mathrm{CH}_{3} \mathrm{NH}_{3} \mathrm{PbI}_{3}$ perovskite solar cells. Energy \& Environmental Science 2015, 8 (3), 910-915.

11. Gonzalez-Pedro, V.; Juarez-Perez, E. J.; Arsyad, W.-S.; Barea, E. M.; FabregatSantiago, F.; Mora-Sero, I.; Bisquert, J. General working principles of $\mathrm{CH}_{3} \mathrm{NH}_{3} \mathrm{PbX}_{3}$ perovskite solar cells. Nano Letters 2014, 14 (2), 888-893.

12. Snaith, H. J.; Abate, A.; Ball, J. M.; Eperon, G. E.; Leijtens, T.; Noel, N. K.; Stranks, S. D.; Wang, J. T.-W.; Wojciechowski, K.; Zhang, W. Anomalous Hysteresis in Perovskite Solar Cells. The Journal of Physical Chemistry Letters 2014, 5 (9), 1511-1515.

13. Kim, H.-S.; Jang, I.-H.; Ahn, N.; Choi, M.; Guerrero, A.; Bisquert, J.; Park, N.-G. Control of $\mathrm{I}-\mathrm{V}$ hysteresis in $\mathrm{CH}_{3} \mathrm{NH}_{3} \mathrm{PbI}_{3}$ perovskite solar cell. The Journal of Physical Chemistry Letters 2015, 6 (22), 4633-4639.

14. Tress, W.; Marinova, N.; Moehl, T.; Zakeeruddin, S. M.; Nazeeruddin, M. K.; Gratzel, M. Understanding the rate-dependent J-V hysteresis, slow time component, and aging in $\mathrm{CH}_{3} \mathrm{NH}_{3} \mathrm{PbI}_{3}$ perovskite solar cells: the role of a compensated electric field. Energy \& Environmental Science 2015, 8 (3), 995-1004.

15. Sanchez, R. S.; Gonzalez-Pedro, V.; Lee, J.-W.; Park, N.-G.; Kang, Y. S.; MoraSero, I.; Bisquert, J. Slow Dynamic Processes in Lead Halide Perovskite Solar Cells. Characteristic Times and Hysteresis. The Journal of Physical Chemistry Letters 2014, 5 
(13), 2357-2363.

16. Unger, E. L.; Hoke, E. T.; Bailie, C. D.; Nguyen, W. H.; Bowring, A. R.; Heumüller, T.; Christoforo, M. G.; McGehee, M. D. Hysteresis and transient behavior in current-voltage measurements of hybrid-perovskite absorber solar cells. Energy \& Environmental Science 2014, 7 (11), 3690-3698.

17. Shen, H.; Jacobs, D. A.; Wu, Y.; Duong, T.; Peng, J.; Wen, X.; Fu, X.; Karuturi, S. K.; White, T. P.; Weber, K.; Catchpole, K. R. Inverted Hysteresis in $\mathrm{CH}_{3} \mathrm{NH}_{3} \mathrm{PbI}_{3}$ Solar Cells: Role of Stoichiometry and Band Alignment. The Journal of Physical Chemistry Letters 2017, 8 (12), 2672-2680.

18. Nemnes, G. A.; Besleaga, C.; Stancu, V.; Dogaru, D. E.; Leonat, L. N.; Pintilie, L.; Torfason, K.; Ilkov, M.; Manolescu, A.; Pintilie, I. Normal and Inverted Hysteresis in Perovskite Solar Cells. The Journal of Physical Chemistry C 2017, 121 (21), 1120711214.

19. Tress, W.; Correa Baena, J. P.; Saliba, M.; Abate, A.; Graetzel, M. Inverted Current-Voltage Hysteresis in Mixed Perovskite Solar Cells: Polarization, Energy Barriers, and Defect Recombination. Advanced Energy Materials 2016, 6 (19), 1600396.

20. Richardson, G.; O'Kane, S. E. J.; Niemann, R. G.; Peltola, T. A.; Foster, J. M.; Cameron, P. J.; Walker, A. B. Can slow-moving ions explain hysteresis in the currentvoltage curves of perovskite solar cells? Energy \& Environmental Science 2016, 9 (4), 1476-1485.

21. Ku, Z.; Rong, Y.; Xu, M.; Liu, T.; Han, H. Full Printable Processed Mesoscopic $\mathrm{CH}_{3} \mathrm{NH}_{3} \mathrm{PbI}_{3} / \mathrm{TiO}_{2}$ Heterojunction Solar Cells with Carbon Counter Electrode. Scientific Reports 2013, 3, 3132.

22. Mei, A.; Li, X.; Liu, L.; Ku, Z.; Liu, T.; Rong, Y.; Xu, M.; Hu, M.; Chen, J.; Yang, Y.; Grätzel, M.; Han, H. A hole-conductor-free, fully printable mesoscopic perovskite solar cell with high stability. Science 2014, 345 (6194), 295-298.

23. Rong, Y.; Hou, X.; Hu, Y.; Mei, A.; Liu, L.; Wang, P.; Han, H. Synergy of ammonium chloride and moisture on perovskite crystallization for efficient printable mesoscopic solar cells. Nature Communications 2017, 8, 14555.

24. Rong, Y.; Ku, Z.; Mei, A.; Liu, T.; Xu, M.; Ko, S.; Li, X.; Han, H. HoleConductor-Free Mesoscopic $\mathrm{TiO}_{2} / \mathrm{CH}_{3} \mathrm{NH}_{3} \mathrm{PbI}_{3}$ Heterojunction Solar Cells Based on Anatase Nanosheets and Carbon Counter Electrodes. The Journal of Physical Chemistry Letters 2014, 5 (12), 2160-4.

25. Heo, J. H.; Han, H. J.; Kim, D.; Ahn, T. K.; Im, S. H. Hysteresis-less inverted $\mathrm{CH}_{3} \mathrm{NH}_{3} \mathrm{PbI}_{3}$ planar perovskite hybrid solar cells with $18.1 \%$ power conversion efficiency. Energy \& Environmental Science 2015, 8 (5), 1602-1608.

26. Yang, D.; Zhou, X.; Yang, R.; Yang, Z.; Yu, W.; Wang, X.; Li, C.; Liu, S.; Chang, 
R. P. H. Surface optimization to eliminate hysteresis for record efficiency planar perovskite solar cells. Energy \& Environmental Science 2016.

27. Gottesman, R.; Lopez-Varo, P.; Gouda, L.; Jimenez-Tejada, Juan A.; Hu, J.; Tirosh, S.; Zaban, A.; Bisquert, J. Dynamic Phenomena at Perovskite/Electron-Selective Contact Interface as Interpreted from Photovoltage Decays. Chem 2016, 1 (5), 776-789.

28. Kim, H.-S.; Park, N.-G. Parameters affecting I-V hysteresis of $\mathrm{CH}_{3} \mathrm{NH}_{3} \mathrm{PbI}_{3}$ perovskite solar cells: effects of perovskite crystal size and mesoporous $\mathrm{TiO}_{2}$ layer. The Journal of Physical Chemistry Letters 2014, 5 (17), 2927-2934.

29. Shao, J.; Yang, S.; Lei, L.; Cao, Q.; Yu, Y.; Liu, Y. Pore Size Dependent Hysteresis Elimination in Perovskite Solar Cells Based on Highly Porous $\mathrm{TiO}_{2}$ Films with Widely Tunable Pores of 15-34 nm. Chem. Mater. 2016, 28 (19), 7134-7144.

30. Zhang, F.; Ma, W.; Guo, H.; Zhao, Y.; Shan, X.; Jin, K.; Tian, H.; Zhao, Q.; Yu, D.; Lu, X.; Lu, G.; Meng, S. Interfacial Oxygen Vacancies as a Potential Cause of Hysteresis in Perovskite Solar Cells. Chemistry of Materials 2016, 28 (3), 802-812.

31. Kim, Y. C.; Jeon, N. J.; Noh, J. H.; Yang, W. S.; Seo, J.; Yun, J. S.; Ho-Baillie, A.; Huang, S.; Green, M. A.; Seidel, J.; Ahn, T. K.; Seok, S. I. Beneficial Effects of $\mathrm{PbI}_{2}$ Incorporated in Organo-Lead Halide Perovskite Solar Cells. Advanced Energy Materials 2016, 6 (4), 1502104.

32. Li, X.; Bi, D.; Yi, C.; Décoppet, J.-D.; Luo, J.; Zakeeruddin, S. M.; Hagfeldt, A.; Grätzel, M. A vacuum flash-assisted solution process for high-efficiency large-area perovskite solar cells. Science 2016, 353 (6294), 58-62.

33. Bag, M.; Renna, L. A.; Adhikari, R. Y.; Karak, S.; Liu, F.; Lahti, P. M.; Russell, T. P.; Tuominen, M. T.; Venkataraman, D. Kinetics of Ion Transport in Perovskite Active Layers and Its Implications for Active Layer Stability. Journal of the American Chemical Society 2015, 137 (40), 13130-13137.

34. Li, C.; Tscheuschner, S.; Paulus, F.; Hopkinson, P. E.; Kießling, J.; Köhler, A.; Vaynzof, Y.; Huettner, S. Iodine Migration and its Effect on Hysteresis in Perovskite Solar Cells. Advanced Materials 2016, 28 (12), 2446-2454.

35. Tiwana, P.; Docampo, P.; Johnston, M. B.; Snaith, H. J.; Herz, L. M. Electron Mobility and Injection Dynamics in Mesoporous $\mathrm{ZnO}, \mathrm{SnO} 2$, and $\mathrm{TiO} 2$ Films Used in Dye-Sensitized Solar Cells. ACS Nano 2011, 5 (6), 5158-5166.

36. Abayev, I.; Zaban, A.; Fabregat-Santiago, F.; Bisquert, J. Electronic conductivity in nanostructured $\mathrm{TiO}_{2}$ films permeated with electrolyte. Physica Status Solidi (a) 2003, 196 (1), R4-R6.

37. Wehrenfennig, C.; Eperon, G. E.; Johnston, M. B.; Snaith, H. J.; Herz, L. M. High Charge Carrier Mobilities and Lifetimes in Organolead Trihalide Perovskites. Advanced Materials 2014, 26 (10), 1584-1589. 
38. Almora, O.; Zarazua, I.; Mas-Marza, E.; Mora-Sero, I.; Bisquert, J.; GarciaBelmonte, G. Capacitive Dark Currents, Hysteresis, and Electrode Polarization in Lead Halide Perovskite Solar Cells. The Journal of Physical Chemistry Letters 2015, 6 (9), 1645-1652.

39. Garcia-Belmonte, G.; Bisquert, J. Distinction between Capacitive and Noncapacitive Hysteretic Currents in Operation and Degradation of Perovskite Solar Cells. ACS Energy Letters 2016, 1 (4), 683-688.

40. Handa, T.; Tex, D. M.; Shimazaki, A.; Wakamiya, A.; Kanemitsu, Y. Charge Injection Mechanism at Heterointerfaces in $\mathrm{CH}_{3} \mathrm{NH}_{3} \mathrm{PbI}_{3}$ Perovskite Solar Cells Revealed by Simultaneous Time-Resolved Photoluminescence and Photocurrent Measurements. The Journal of Physical Chemistry Letters 2017, 8 (5), 954-960.

41. Zarazua, I.; Bisquert, J.; Garcia-Belmonte, G. Light-Induced Space-Charge Accumulation Zone as Photovoltaic Mechanism in Perovskite Solar Cells. The Journal of Physical Chemistry Letters 2016, 7 (3), 525-528. 


\title{
Supporting Information for
}

\section{Tunable hysteresis effect for perovskite solar cells}

\author{
Yaoguang Rong ${ }^{1 \dagger}$, Yue Hu${ }^{1 \dagger}$, Sandheep Ravishankar ${ }^{2 \dagger}$, Huawei Liu ${ }^{1}$, Xiaomeng
}

Hou $^{1}$, Yusong Sheng ${ }^{1}$, Anyi Mei ${ }^{1}$, Qifei Wang ${ }^{1}$, Daiyu Li ${ }^{1}$, Mi Xu ${ }^{1}$, Juan Bisquert ${ }^{2}$, $3^{*}$, Hongwei Han ${ }^{*}$

${ }^{1}$ Michael Grätzel Center for Mesoscopic Solar Cells, Wuhan National Laboratory for Optoelectronics, School of Optical and Electronic Information, Huazhong University of Science and Technology, Wuhan 430074, Hubei, PR China

${ }^{2}$ Institute of Advanced Materials (INAM), Universitat Jaume I, 12006 Castelló, Spain

${ }^{3}$ Department of Chemistry, Faculty of Science, King Abdulaziz University, Jeddah, Saudi Arabia

†These authors contributed equally to this work.

*Email: hongwei.han@mail.hust.edu.cn, bisquert@uji.es 


\section{Experiment}

\section{Materials}

Unless stated otherwise, all materials were purchased from Sigma-Aldrich or Acros Organics and used as received. $\mathrm{CH}_{3} \mathrm{NH}_{3} \mathrm{I}$ was synthesized and purified according to literature procedures ${ }^{1}$. The perovskite precursor solution was prepared by dissolving $573.0 \mathrm{mg} \mathrm{PbI} 2,197.5 \mathrm{mg} \mathrm{CH}_{3} \mathrm{NH}_{3} \mathrm{I}$ and $13.70 \mathrm{mg}$ 5-AVAI in $1.0 \mathrm{ml} \gamma$-butyrolactone (GBL), and stirred at $70{ }^{\circ} \mathrm{C}$ for $30 \mathrm{~min}$. The $\mathrm{TiO}_{2}, \mathrm{ZrO}_{2}$ and carbon pastes were prepared as previously reported ${ }^{2-3}$.

\section{Device fabrication}

Unless stated otherwise, the whole device fabrication process was carried out under ambient conditions (RH25-35\%). The FTO-coated glass substrates (Tec15, Pilkington) were first etched by laser and cleaned by ultrasonication with detergent, deionized water, acetone and ethanol. The $\mathrm{c}-\mathrm{TiO}_{2}$ layer was then deposited on the patterned substrates by aerosol spray pyrolysis as follow: The patterned FTO substrate was placed on a hotplate with a mask, as shown in Figure S1, and heated up to $450{ }^{\circ} \mathrm{C}$. A titanium diisopropoxide bis(acetylacetonate) solution was diluted in ethanol (1:39, volume ratio), and kept in a glass sprayer which was purchased from Sigma-Aldrich. The distance between the sprayer and the hotplate was $\sim 10 \mathrm{~cm}$, and the spraying angle was 60-70 degree. The carrier gas was oxygen with a flow rate of $\sim 10 \mathrm{ml} / \mathrm{s}$. The sprayer moved at a speed of $\sim 10 \mathrm{~cm} / \mathrm{s}$, along the red line marked spraying path in Figure S1. After spraying, the $\mathrm{c}-\mathrm{TiO}_{2}$ coated FTO substrate was sintered at $450{ }^{\circ} \mathrm{C}$ for $30 \mathrm{~min}$. After cooling to room temperature (RT, 25 ${ }^{\circ} \mathrm{C}$ ), a $1 \mu \mathrm{m}$-thick mesoporous $\mathrm{TiO}_{2}$ layer, a $2 \mu \mathrm{m}$-thick $\mathrm{ZrO}_{2}$ spacer layer and a $10 \mu \mathrm{m}$ thick carbon layer were screen-printed on the substrates layer by layer. $\mathrm{The}^{\mathrm{TiO}} 2$ layer and $\mathrm{ZrO}_{2}$ layer were sintered at $450{ }^{\circ} \mathrm{C}$ for $30 \mathrm{~min}$, and the carbon layer was sintered at $400{ }^{\circ} \mathrm{C}$ for $30 \mathrm{~min}$, forming the mesoporous triple-layer based scaffold. After cooling to room temperature, the perovskite precursor solution was infiltrated by drop casting via the top of the carbon layer. After drying at $50{ }^{\circ} \mathrm{C}$ for one hour, the printable mesoscopic PSCs were obtained.

\section{Characterization}

The thickness of the films was measured by a profilometer (DektakXT, Bruker). The atomic force microscope (AFM) measurements were performed on SPM9700 (Shimadzu). Photocurrent-density voltage $(J-V)$ curves were characterized with a Keithley 2400 source meter and a Newport solar simulator (model 91192). The power of the simulated light was calibrated to $100 \mathrm{~mW} \mathrm{~cm}^{-2}$ using a Newport Oriel PV reference cell (model 91150V). The active area of the device is about $0.8 \mathrm{~cm}^{2}$ and a black mask with a circular aperture $\left(0.126 \mathrm{~cm}^{2}\right)$ was applied for $J-V$ measurements. The $J-V$ testing was performed with forward and backward scan directions at a scan rate of $250 \mathrm{mV} \mathrm{s}^{-1}, 100$ $\mathrm{mV} \mathrm{s}^{-1}, 50 \mathrm{mV} \mathrm{s}^{-1}$, and $25 \mathrm{mV} \mathrm{s}^{-1}$ (sweep delay time of $100 \mathrm{~ms}$ ). Before $J$ - $V$ scans, the 
devices were usually subjected to the simulate sun light for several minutes. All the measurements were carried out at $\mathrm{RH} 25-35 \%$ and room temperature.

Table S1 Dependence of hysteresis effect on spraying cycles of c- $\mathrm{TiO}_{2}$ and UV-Ozone treatment. The hysteresis effect has been defined as normal, free and inverted.

\begin{tabular}{ccccc}
\hline Condition & $\mathrm{c}-\mathrm{TiO}_{2} \times 1$ & $\mathrm{c}-\mathrm{TiO}_{2} \times 2$ & $\mathrm{c}-\mathrm{TiO}_{2} \times 3$ & $\mathrm{c}-\mathrm{TiO}_{2} \times 4$ \\
\hline UV-Ozone 0 min & Normal & Normal & Normal & Normal \\
UV-Ozone 2.5 & Inverted & Free & Normal & Normal \\
min & & & & \\
UV-Ozone 10 & Inverted & Inverted & Normal & Normal \\
min & & & & \\
UV-Ozone 30 & Inverted & Inverted & Free & Normal \\
min & & & & \\
\hline
\end{tabular}


Table S2 Series resistance and shunt resistance of hysteresis-normal, hysteresis-free, and hysteresis-inverted devices measured at different scan directions and scan rates $\left(R_{\mathrm{S}}: \Omega\right.$ $\left.\mathrm{cm}^{2} ; R_{\mathrm{SH}}: \mathrm{k} \Omega \mathrm{cm}^{2}\right)$.

\begin{tabular}{|c|c|c|c|c|c|c|c|c|c|c|c|c|c|c|c|c|}
\hline & \multicolumn{2}{|c|}{$\begin{array}{l}\text { F-R } \\
\text { can }\end{array}$} & \multicolumn{2}{|c|}{$\begin{array}{l}\text { R-F } \\
\text { can }\end{array}$} & \multicolumn{2}{|c|}{$\begin{array}{l}\text { F-R } \\
\text { can }\end{array}$} & \multicolumn{2}{|c|}{$\begin{array}{l}\text { R-F } \\
\text { can }\end{array}$} & \multicolumn{2}{|c|}{$\begin{array}{l}\text { F-R } \\
\text { can }\end{array}$} & \multicolumn{2}{|c|}{$\begin{array}{l}\text { R-F } \\
\text { scan }\end{array}$} & \multicolumn{2}{|c|}{$\begin{array}{l}\text { F-R } \\
\text { scan }\end{array}$} & \multicolumn{2}{|c|}{$\begin{array}{l}\text { R-F } \\
\text { scan }\end{array}$} \\
\hline & \multicolumn{4}{|c|}{$25 \mathrm{mV} \mathrm{s}^{-1}$} & \multicolumn{4}{|c|}{$50 \mathrm{mV} \mathrm{s}^{-1}$} & \multicolumn{4}{|c|}{$100 \mathrm{mV} \mathrm{s}^{-1}$} & \multicolumn{4}{|c|}{$250 \mathrm{mV} \mathrm{s}^{-1}$} \\
\hline & $R_{\mathrm{S}}$ & $\begin{array}{l}R_{\mathrm{S}} \\
\mathrm{H}\end{array}$ & $R$ & $\begin{array}{r}R \\
\mathrm{H}\end{array}$ & $R$ & $\begin{array}{r}R \\
\text { н }\end{array}$ & $R$ & $\begin{array}{r}R \\
\mathrm{H}\end{array}$ & $R$ & $\begin{array}{r}R \\
\mathrm{H}\end{array}$ & $R_{s}$ & $\begin{array}{c}R_{S} \\
\mathrm{H}\end{array}$ & $R_{S}$ & $\begin{array}{c}R_{\mathrm{S}} \\
\mathrm{H}\end{array}$ & $R_{S}$ & $\begin{array}{c}R_{S} \\
\mathrm{H}\end{array}$ \\
\hline & $\begin{array}{r}6 \\
27 \pm 0\end{array}$ & 0. & 6. & 0 & 6 & 0 & 5 & 0 & 5 & 0 & 5 & 0. & 5. & 0. & 5. & $\begin{array}{r}0 . \\
20 \pm 0\end{array}$ \\
\hline al & .24 & .16 & .30 & .40 & .21 & .21 & .19 & .10 & .14 & .34 & .12 & .26 & .20 & .27 & .15 & .07 \\
\hline & & & 7. & 0 & 6 & 0 & 7 & 1 & 6 & 0 & 6. & 0 & 6 & 0 . & 6. & $43 \pm 0$ \\
\hline 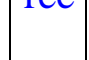 & .13 & .27 & .26 & .4 & .21 & .22 & .18 & .48 & .1 & .23 & .16 & 24 & .21 & .27 & .02 & .21 \\
\hline & & 0 & 6. & 0 & 6 & 0 & 5 & 0 & 5 & $\begin{array}{r}0 \\
71 \pm\end{array}$ & 5 & $\begin{array}{r}0 . \\
49 \pm 0\end{array}$ & 5 & $\begin{array}{r}0 . \\
56 \pm 0\end{array}$ & $52 \pm 0$ & $29 \pm 0$ \\
\hline ted & .22 & .13 & .26 & .14 & .19 & .19 & .17 & .25 & .14 & .34 & .12 & .26 & .20 & .35 & .13 & .20 \\
\hline
\end{tabular}


Table S3 Fitting decay times of time-resolved photoluminescence (TRPL) spectra. The semi-devices were fabricated as $\mathrm{FTO} / \mathrm{c}-\mathrm{TiO}_{2} / \mathrm{m}-\mathrm{TiO}_{2} / \mathrm{ZrO}_{2} /$ Perovskite, and the c$\mathrm{TiO}_{2}$ layers were sprayed with 1-4 cycles.

\begin{tabular}{|c|c|c|}
\hline $\begin{array}{c}\text { c- TiO } \\
\text { cycle }\end{array}$ & $\tau_{\mathbf{1}}(\mathbf{n s})$ & $\boldsymbol{\tau}_{\mathbf{2}}(\mathbf{n s})$ \\
\hline \multirow{3}{*}{1} & 1.12 & 33.91 \\
\hline \multirow{2}{*}{2} & 1.16 & 36.48 \\
\hline \multirow{2}{*}{2} & 1.10 & 36.89 \\
\hline \multirow{2}{*}{3} & 2.16 & 51.29 \\
\hline \multirow{2}{*}{3} & 1.29 & 37.90 \\
& 1.11 & 38.39 \\
\hline \multirow{2}{*}{4} & 1.23 & 39.95 \\
& 1.19 & 37.68 \\
\hline & 1.18 & 37.58 \\
\hline & 1.46 & 40.05 \\
\hline
\end{tabular}

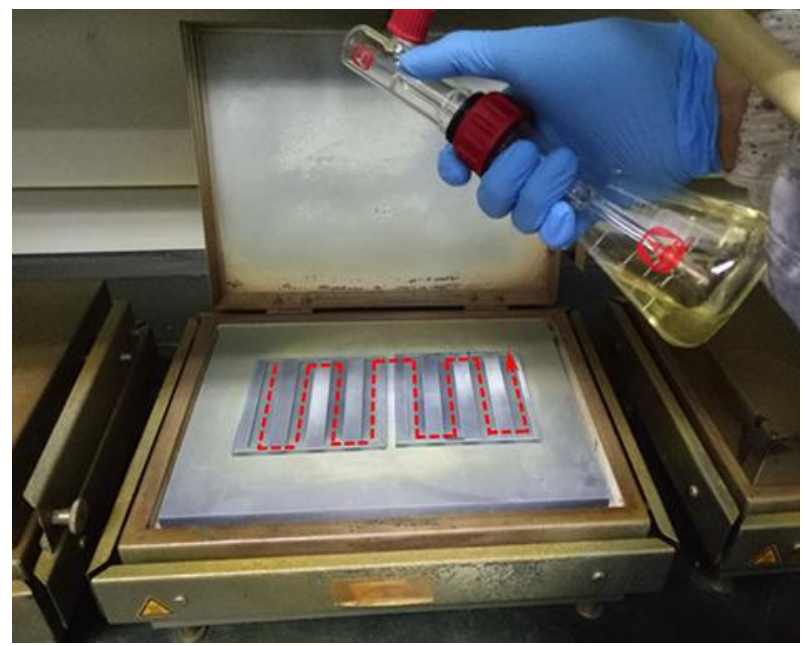

Fig. S1 The spraying deposition process of the compact- $\mathrm{TiO}_{2}\left(\mathrm{c}-\mathrm{TiO}_{2}\right)$ layer. The sprayer is purchased from Sigma-Aldrich, and the carrier gas is $\mathrm{N}_{2}$ with a flow rate of $\sim 10 \mathrm{ml} / \mathrm{s}$. 

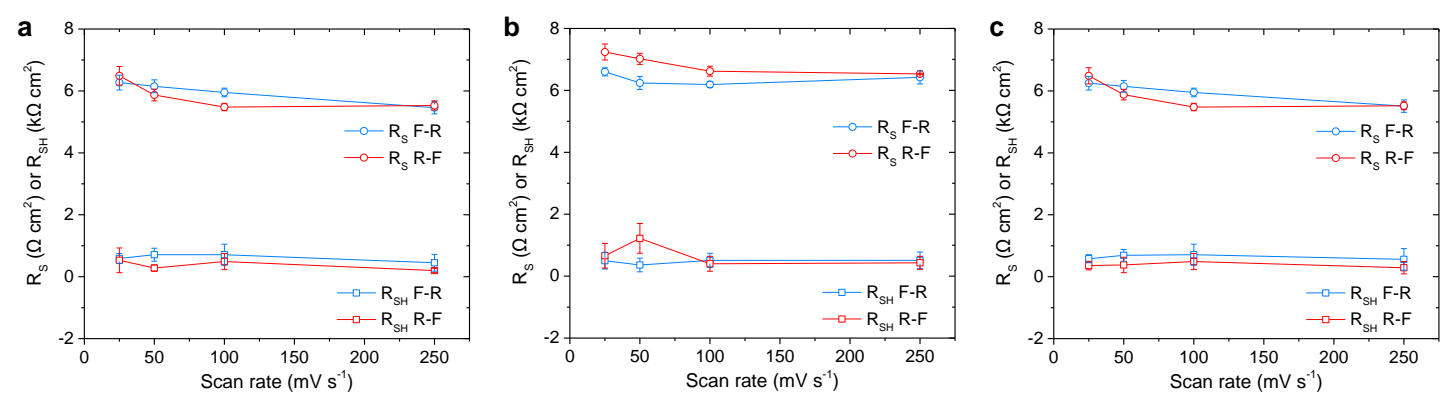

Fig. S2 The dependence of $\mathrm{R}_{\mathrm{S}}$ and $\mathrm{R}_{\mathrm{SH}}$ on scan rates for hysteresis-normal, hysteresisfree and hysteresis-inverted devices.
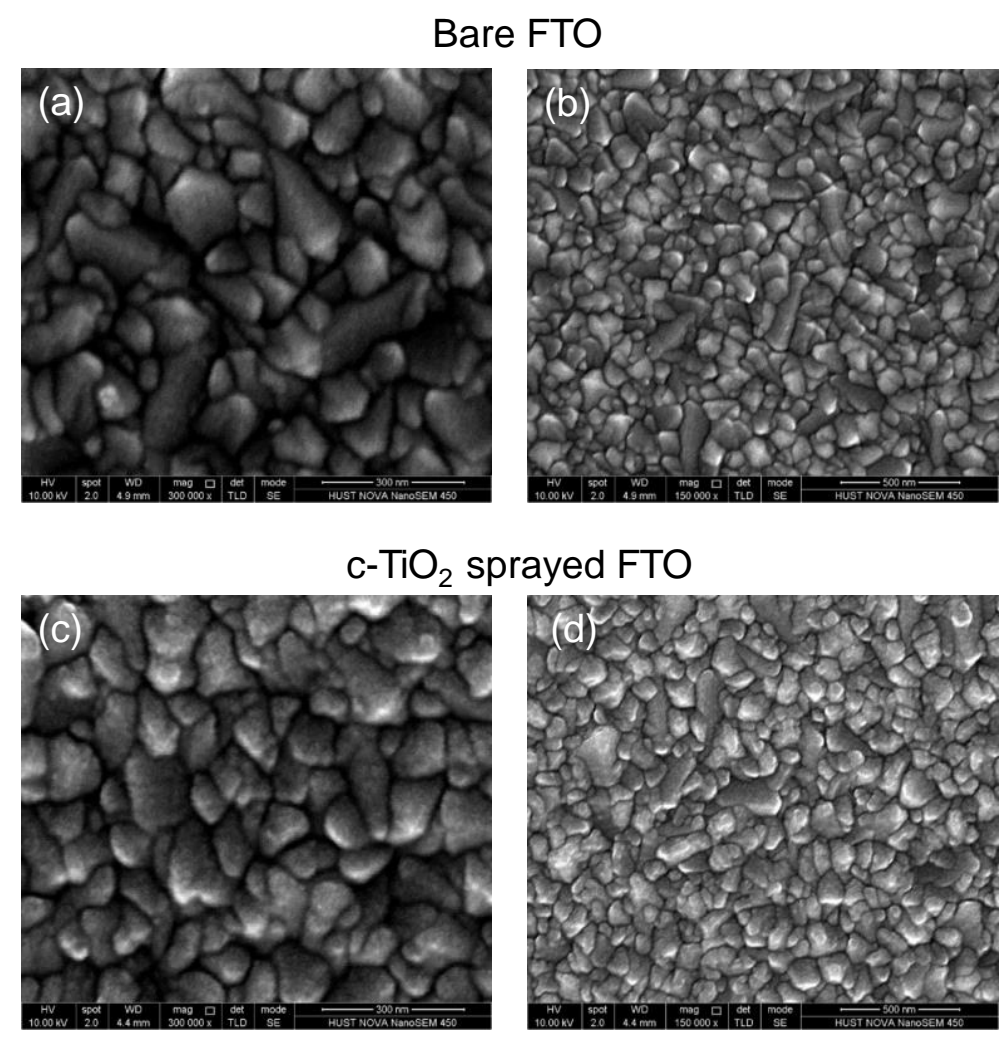

Fig. S3 Surface morphology characterizations. The SEM images of the bare FTO substrate $(\mathrm{a}, \mathrm{b})$ and $\mathrm{c}-\mathrm{TiO}_{2}$ sprayed (two cycles) FTO substrate (c, d). 


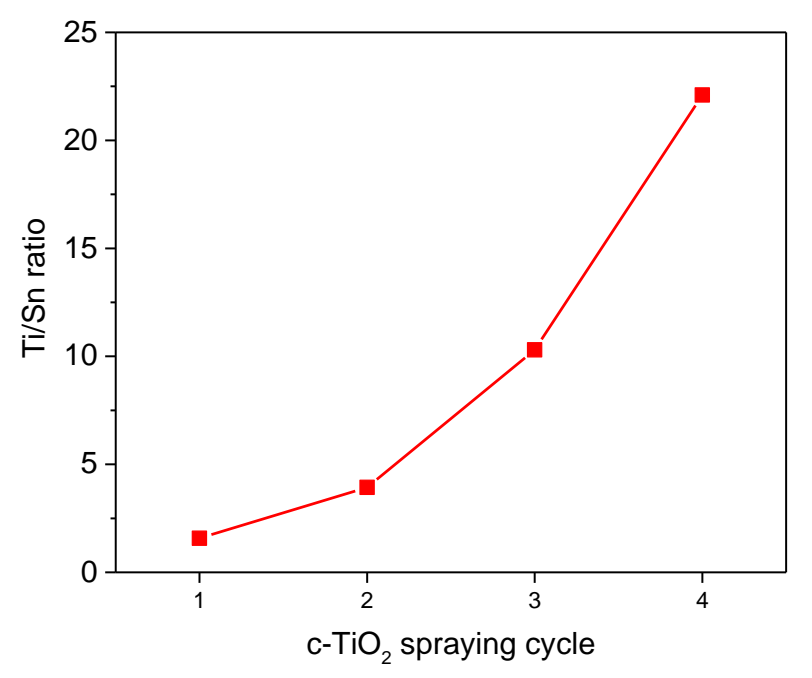

Fig. S4 XPS results of FTO substrates sprayed with 1-4 cycles of c- $\mathrm{TiO}_{2}$ layer. The measurements were performed using the same accumulation time, and the Ti and Sn peak areas are compared.

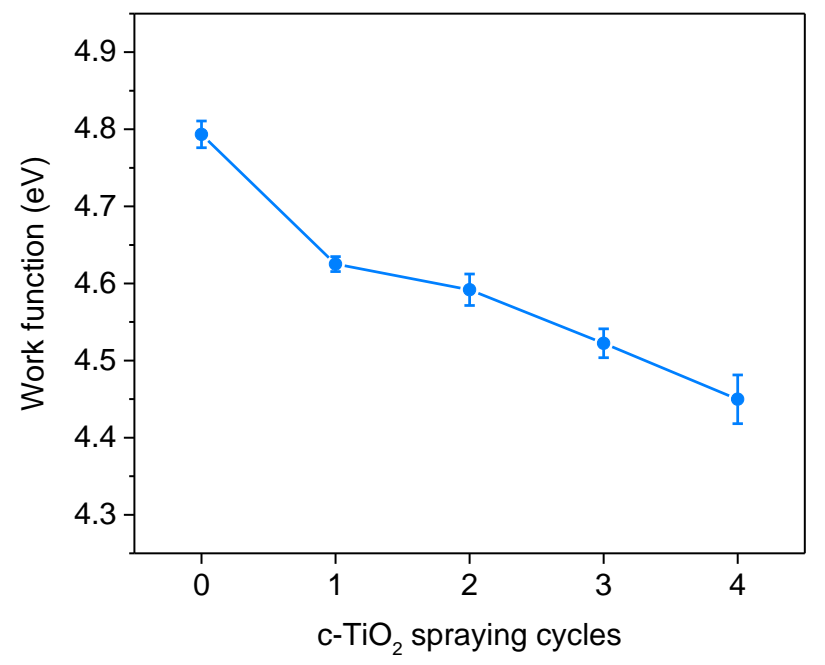

Fig. S5 Work function of FTO substrates sprayed with 0-4 cycle of c- $\mathrm{TiO}_{2}$ layer (Error bars represent $s . d$. calculated from five values obtained with the same testing parameters). 


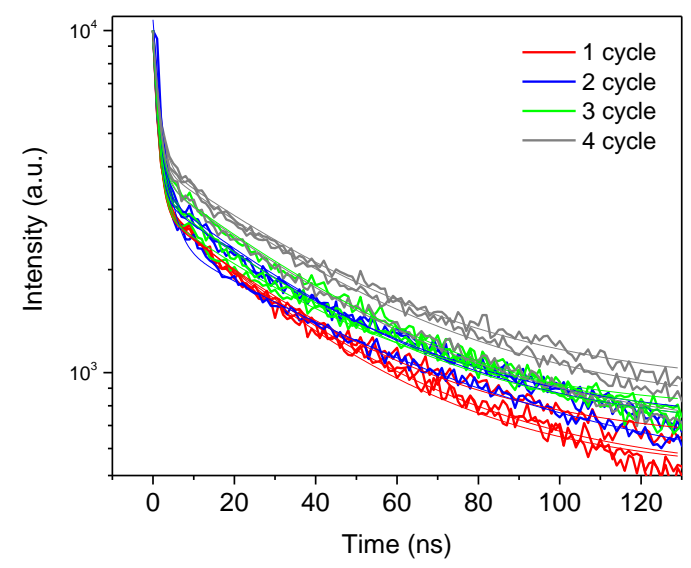

Fig. S6 Time-resolved photoluminescence (TRPL) spectra of FTO/c-TiO $/ 2 / m-\mathrm{TiO}_{2}$ $/ \mathrm{ZrO}_{2} /$ Perovskite semi-devices. The $\mathrm{c}-\mathrm{TiO}_{2}$ layers were sprayed with 1-4 cycles. For each spraying cycle, three samples were prepared and characterized.
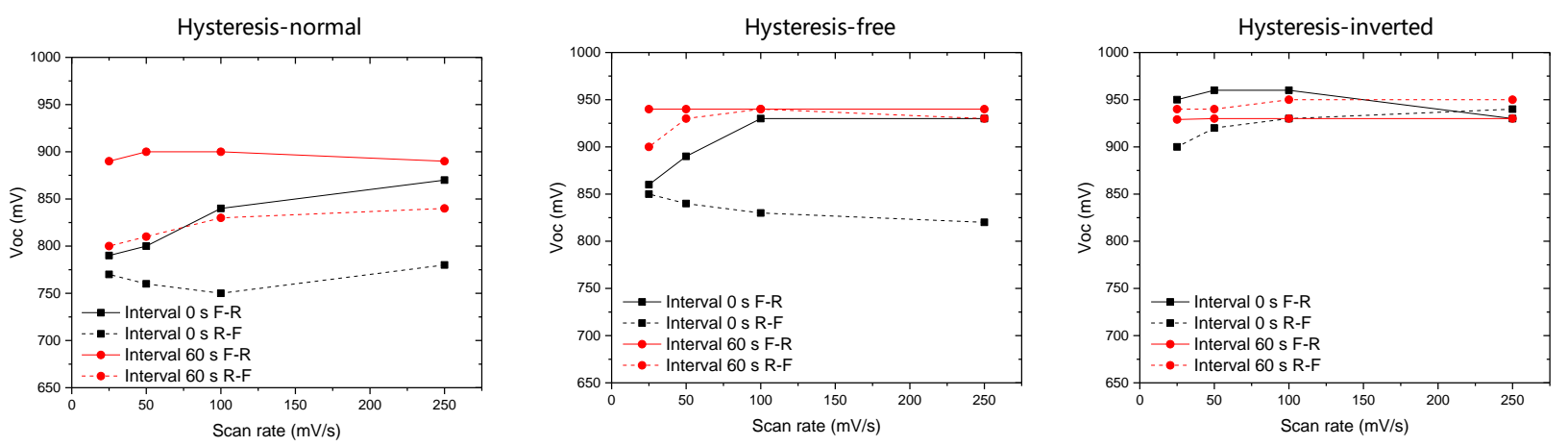

Fig. S7 Dependence of $V_{\mathrm{OC}}$ on scanning rates. The hysteresis-normal, -free and -inverted devices were continuously scanned with an interval of $0 \mathrm{~s}$ or discontinuously scanned with an interval of $60 \mathrm{~s}$ at $250 \mathrm{mV} \mathrm{s}^{-1}, 100 \mathrm{mV} \mathrm{s}^{-1}, 50 \mathrm{mV} \mathrm{s}^{-1}$ and $25 \mathrm{mV} \mathrm{s}^{-1}$ firstly in F-R direction, and then in R-F direction.

\section{References}

1. Etgar, L.; Gao, P.; Xue, Z.; Peng, Q.; Chandiran, A. K.; Liu, B.; Nazeeruddin, M. K.; Grätzel, M. Mesoscopic $\mathrm{CH}_{3} \mathrm{NH}_{3} \mathrm{PbI}_{3} / \mathrm{TiO}_{2}$ heterojunction solar cells. Journal of the American Chemical Society 2012, 134 (42), 17396-17399.

2. Ku, Z.; Rong, Y.; Xu, M.; Liu, T.; Han, H. Full Printable Processed Mesoscopic $\mathrm{CH}_{3} \mathrm{NH}_{3} \mathrm{PbI}_{3} / \mathrm{TiO}_{2}$ Heterojunction Solar Cells with Carbon Counter Electrode. Scientific Reports 2013, 3, 3132.

3. Mei, A.; Li, X.; Liu, L.; Ku, Z.; Liu, T.; Rong, Y.; Xu, M.; Hu, M.; Chen, J.; Yang, Y.; Grätzel, M.; Han, H. A hole-conductor-free, fully printable mesoscopic perovskite solar cell with high stability. Science 2014, 345 (6194), 295-298. 


\section{Chapter 6}

\section{Publication 3}

Influence of Charge Transport Layers on Open-Circuit Voltage and Hysteresis in Perovskite Solar Cells

Ravishankar, S.; Gharibzadeh, S.; Roldán-Carmona, C.; Grancini, G.; Lee, Y.; Ralaiarisoa, M.; Asiri, A. M.; Koch, N.; Bisquert, J.; Nazeeruddin, M. K. Influence of Charge Transport Layers on Open-Circuit Voltage and Hysteresis in Perovskite Solar Cells. Joule 2018, 2 (4), 788-798. Reprinted with permission, Copyright 2018, Elsevier Inc.

\section{Candidate's contribution}

\begin{tabular}{|c|c|}
\hline Nature of Contribution & Extent of Contribution \\
\hline $\begin{array}{l}\text { - Carried out IS and hysteresis } \\
\text { measurements } \\
\text { - Contributed to interpretation of the } \\
\text { results } \\
\text { - Wrote first draft of the manuscript } \\
\text { - Contributed to the reply to the } \\
\text { referees }\end{array}$ & $40 \%$ \\
\hline
\end{tabular}

\section{Introduction}

The critical dependence of the hysteresis patterns on the nature of the ETL developed over the last two chapters is further explored to identify its role in the obtained $V_{o c}$ for high efficiency perovskite solar cells. A systematic investigation using ETLs with different workfunctions has very little effect on the $V_{o c}$, which has severe implications on the existing interpretation of the role of the electric field in photogenerated carrier transport in PSCs.

\section{Published manuscript}




\section{Influence of charge transport layers on open-circuit voltage and hysteresis in perovskite solar cells}

Sandheep Ravishankar, ${ }^{1 \square}$ Saba Gharibzadeh, ${ }^{2,3 \square}$ Cristina Roldán-Carmona, ${ }^{* 2}$ Giulia Grancini, ${ }^{2}$ Yonghui Lee ${ }^{2}$, Maryline Ralaiarisoa ${ }^{4,5}$, Abdullah M. Asiri, ${ }^{6}$ Norbert Koch $^{4,5}$, Juan Bisquert ${ }^{* 1,7}$ and Mohammad Khaja Nazeeruddin*2

${ }^{1}$ Institute of Advanced Materials (INAM), Universitat Jaume I, 12006 Castelló, Spain

${ }^{2}$ GMF, Institute of Chemical Sciences and Engineering, EPFL Valais, Rue de L'Indutrie, 1951 Sion, Switzerland.

${ }^{3}$ Department of Physics, Tarbiat Modares University, P.O. Box 14115-175, Tehran-Iran

${ }^{4}$ Humboldt-Universität zu Berlin, Institut für Physik \& IRIS Adlershof, Berlin, Germany

5 Helmholtz-Zentrum Berlin für Materialien und Energie, Devision Renewable Energies, Berlin, Germany

${ }^{6}$ Center of Excellence for Advanced Materials Research (CEAMR), King Abdulaziz University, P. O. Box 80203, Jeddah 21589, Saudi Arabia

${ }^{7}$ Department of Chemistry, Faculty of Science, King Abdulaziz University, Jeddah, Saudi Arabia

${ }^{\square}$ Equal contributing first authors.

*Corresponding authors E-mail: mdkhaja.nazeeruddin@epfl.ch, bisquert@uji.es, cristina.roldancarmona@epfl.ch

Summary

Perovskite materials have experienced an impressive improvement in photovoltaic performance due to their unique combination of optoelectronic properties. Their remarkable progression, facilitated by the use of different device architectures, compositional engineering and processing methodologies, contrast with the lack of understanding of the materials properties and interface phenomena. Here we directly target the interplay between the charge transporting layers (CTL) and open-circuit potential $\left(V_{o c}\right)$ in the operation mechanism of the state-of-the-art $\mathrm{CH}_{3} \mathrm{NH}_{3} \mathrm{PbI}_{3}$ solar cells. Our results suggest that the $V_{o c}$ is controlled by the splitting of quasi-Fermi levels and recombination inside the perovskite, rather than being governed by any internal electric field established by the difference in the CTL's work functions. In addition, we provide novel insights into the hysteretic origin in PSCs, identifying the nature of the contacts as a critical factor in defining the chargeaccumulation at its interface, leading to either ionic, electronic or mixed ionic- 


\section{electronic accumulation.}

\section{Introduction}

Despite the impressive improvement in device performance, ${ }^{4-11}$ the operating mechanism that governs the high $V_{o c}$ and short circuit current $\left(J_{s c}\right)$ in perovskite photovoltaics remains unclear. Perovskite solar cells (PSCs) suffer from variations in the current-voltage behaviour based on specific experimental parameters, including prebiasing, light intensity, scan direction and rate, the set of which have been referred to as dynamic hysteresis. ${ }^{9}$ Early studies considered such dynamic hysteresis ${ }^{10}$ as the result of a charge collection limitation, conceiving device models in which the transport of chargecarriers arises from the built-in electric field, generated through the perovskite layer and modified by the mobile ions. ${ }^{12-13}$ Nevertheless, the recent progression in materials processing ${ }^{13-14}$ suggests a different scenario, as the state-of-the-art devices possess excellent transport and collection properties, with little to no variation in the photocurrent throughout the scan. In addition, a recent correlation between $\mathrm{TiO}_{2} /$ perovskite interface and the output voltage in perovskite solar cells (PSCs) ${ }^{15-16}$ opens the question of the role of interfaces in the device operation, calling for deeper investigations in their operation mechanism, with special emphasis on how variations in recombination and/or accumulation at the interfaces affect the hysteretic trends. In this article, we answer some of these questions by investigating the role of the contacts in the transport and recombination of state-of-the-art PSCs. Devices with identical architecture but modified electron transporting layer (ETL) interface have been examined, using as device configuration FTO/ ETL / $\mathrm{CH}_{3} \mathrm{NH}_{3} \mathrm{PbI}_{3}$ / Spiro-OMeTAD /Au architecture, where different contacts have been carefully chosen: i) $\mathrm{FTO}_{\text {compact-TiO}} / \mathrm{meso}^{-\mathrm{TiO}_{2}}$; ii) FTO/compact-TiO $\mathrm{O}_{2}$ and iii) FTO electrode (ETL-free cell).

\section{Results}

Figure 1A presents a schematic diagram of the cell architecture along with the crosssectional scanning electron microscopy (SEM) images in Figure 1B obtained for each layout. In all cases, the perovskite thickness is $\sim 300 \mathrm{~nm}$, including the mesoporous based configuration. Interestingly, similar micro-structure and layer morphology could be observed for all architectures, independently from the electron selective contact, as revealed in Figure 1C. In addition, the optical properties of the perovskite films and the X-ray diffraction (XRD) patterns (Figure 1D) were also preserved, showing identical absorption spectra and diffraction patterns corresponding to $\mathrm{CH}_{3} \mathrm{NH}_{3} \mathrm{PbI}_{3}$ tetragonal phase $^{17}$ (see also Figure S1). Overall, these results confirm the formation of perovskite films with comparable crystalline quality and optical properties, which is of extreme importance in the upcoming comparative analysis.

The current-voltage $(J-V)$ curves and the external quantum efficiency $(E Q E)$ of the as prepared solar cells were also analysed. Figures $2 \mathrm{~A}$ and $2 \mathrm{~B}$ show the typical performance 
of three representative cells corresponding to each device configuration (the characteristic solar cells parameters are reported in Table 1). In agreement with previous works, cells employing a mesoscopic architecture exhibit the best photovoltaic performance (PCE = $18.75 \%)$, followed by the planar cells $(\mathrm{PCE}=16.25 \%)$ and the ETL-free devices (PCE = $14.03 \%$ ). Interestingly, $J_{s c}$ hardly changed with the cell configuration, leading to values over $21 \mathrm{~mA} \cdot \mathrm{cm}^{2}$ in all cases. These results agree with the EQE spectra exhibited in Figure 2B, which demonstrates a high photon-to-current conversion over $80 \%$ for the entire visible spectra, even for the ETL-free device. Nevertheless, the most remarkable value is obtained for the $V_{o c}$, remaining close to $1 V$ in all cases. Such a large $V_{o c}$ obtained for the ETL-free cells is in contradiction to previous seminal works ${ }^{12}$ where a large reduction upon removal of the $\mathrm{TiO}_{2}$ layer $(\sim 0.75 \mathrm{~V})$ was observed and attributed to a reduced builtin potential, generally associated to the non-selectivity of the contact. More recently, a similar behaviour was also observed by Juarez-Perez et al. but charge collection was severely reduced, in contrast to our result. ${ }^{18}$ Figure $2 \mathrm{C}, 2 \mathrm{D}$ and Table 1 summarize the photovoltaic performance obtained from more than 40 devices. As deduced from the data, the contact layer is mostly affecting the FF of the cell, which decreases considerably when using a less-selective FTO contact, while $J_{s c}$ and $V_{o c}$ remain very similar. Comparable results have been also observed for cells containing the mixed cation/mixed halide $\left(\mathrm{FAPbI}_{3}\right)_{0.85}\left(\mathrm{MAPbBr}_{3}\right)_{0.15}$ perovskite $(\mathrm{FA}=$ formamidinium), as is illustrated in Figure S2 (see also Table S1). Noteworthy, although slight differences in $J_{s c}$ and $V_{o c}$ can be observed for this perovskite composition, a remarkable high $V_{o c}$ average value over $0.8 \mathrm{~V}$ (with a maximum of $0.96 \mathrm{~V}$ ) is obtained for the ETL-free cell, further generalizing our previous observations.

To verify the electronic structure of the different contact layers, we analyzed them by ultraviolet photoelectron spectroscopy (UPS). The results are presented in Figure S3 and the extracted work functions are also illustrated in Figure 2D, in comparison with the $V_{o c}$. As expected, the FTO layer has the highest work function $(-5.22 \mathrm{eV})$, followed by the blocking $\mathrm{TiO}_{2}(-4.36 \mathrm{eV})$ and the mesoporous $\mathrm{TiO}_{2}$ layer $(-4.33 \mathrm{eV})$. Surprisingly, no apparent relation between the obtained $V_{o c}$ and the work function of the contact can be deduced. This is also observed when inserting a different ETL material such as compact$\mathrm{SnO}_{2}$ layer $\left(\mathrm{WF}=-4.93 \mathrm{eV}\right.$ ), which also gives unexpected high $V_{o c}$ values close to $1 \mathrm{~V}$ (see Figure S4).

To get insight into the electronic operation of the contacts, we investigated the hysteresis features and capacitive nature of these devices via impedance spectroscopy (IS). Figure 3A shows the $J$ - $V$ curves obtained for the three systems measured under scan rates ranging from 10 to $200 \mathrm{mV} / \mathrm{s}$. In all cases, the hysteresis loop between forwardreverse (FR) scans 'opens up' and scales with the scan rate, as typically occurs in a capacitive discharge superimposed onto the steady-state curve. We note that this effect is maximized in the planar $\mathrm{c}-\mathrm{TiO}_{2}$ cells but minimized for the mesoscopic architecture, suggesting meso- $\mathrm{TiO}_{2}$ as the main factor responsible for reducing the overall hysteresis, like often reported in literature. ${ }^{19}$ However, upon removal of the $\mathrm{c}-\mathrm{TiO}_{2}$ layer, the 
hysteresis is again reduced except for very fast scans, denoting $\mathrm{c}-\mathrm{TiO}_{2} /$ perovskite as the real dominant interface (see Figure S5). ${ }^{20}$ Surprisingly, the FTO/perovskite interface also showed strong capacitive trends, indicating a similar mechanism.

\section{Discussion}

According to established photovoltaic knowledge, when a semiconductor light absorber sandwiched between two contacts of very different work function comes to equilibrium, the difference of work functions implies a built in electrical field that has a major role in the transport and extraction of charge. This is usually termed the $\mathrm{p}-\mathrm{i}-\mathrm{n}$ model. On the other hand, 25 years of study of dye-sensitized solar cells and similar devices clearly showed that many solar cells absorb the difference of work functions at the absorber/contact interface, and the electrical field in the device plays a minor role. ${ }^{21}$ Here we show that this is a main principle of operation of the perovskite solar cell, since the $V_{o c}$ remains stable over a striking modification of work function of the electron selective contact that has the same variation as the $\mathrm{V}_{\text {oc }}$ itself, as shown in Fig. 2d. Independently of the built-in electric field across the perovskite, mesoporous morphology, and the difference of work functions across the interfaces, each selective contact will follow the Fermi level of the respective carrier, implying that the main control of open-circuit voltage is established by recombination. ${ }^{22}$ This view of the device is furthermore consistent with the results of Kelvin probe force microscopy (KPFM) on cross sections of planar methylammonium lead iodide that show a flat potential distribution in the absorber layer while most of the potential drops are located at selective contact interfaces. ${ }^{23}$

Under cycling conditions of $10 \mathrm{mV} / \mathrm{s}$, our systems show high $V_{o c}$ values for both FR and RF scans. This opposes the general assumption that a built-in electric field generated by the difference in the work functions of the two contacts mediates the charge collection. On the contrary, it demonstrates that the built-in electric field is not a major factor governing the extraction of carriers, while carrier transport to contacts is mainly due to diffusion, probably granted by the extremely large diffusion length in these materials. As large charge accumulation combining ionic and electronic charge occurs at the outer interfaces, for state-of-the-art devices, hysteretic variations can be directly correlated to the nature of the perovskite crystal in contact with the selective layers, which further underlines the importance of understanding the capacitive trends in these devices.

Figure 3B presents the evolution of the capacitance obtained for each device configuration as a function of frequency (in the dark), at different applied voltages. In general, the capacitance shows a stable plateau at high frequency due to dielectric relaxation in the bulk, and low frequency large values that grow further with the applied voltage, directly probing the contact properties. Importantly, the low frequency value increases over 2 orders of magnitude from $10 \mu \mathrm{F}$ at low frequencies $(\sim 100 \mathrm{mHz})$ to a maximum capacitance of $10 \mathrm{mF}$ for $\sim 1 \mathrm{~V}$. We interpret this capacitance as an accumulation layer of ionic (cations) and electronic carriers (holes) at the $\mathrm{c}-\mathrm{TiO}_{2}$ contact with the perovskite ${ }^{24}$. The capacitance for ion-accumulation is described by the Guoy- 
Chapman model, whose voltage-dependence for voltages above $k_{B} T / q$ is given by

$$
C=\left(\frac{z^{2} q^{2} n_{i}^{0} \varepsilon_{r} \varepsilon_{0}}{2 k_{B} T}\right)^{1 / 2} \cdot \exp \left(\frac{z q V}{m_{C} k_{B} T}\right)
$$

with $Z$ being the charge number of the ions, $q$ the electronic charge, $n_{i}^{0}$ the ionic concentration in the bulk, and $\varepsilon_{r}$ and $\varepsilon_{0}$ the relative and vacuum dielectric constants respectively. $m_{C}$ is a capacitive exponent with the value 2 . However, a similar equation is also used for the accumulation of majority carriers at the interface ${ }^{25}$, again with $m_{C}=2$, hindering the distinction between ionic and electronic accumulation through only capacitance measurements. In our case, there is a difference in the way the low frequency capacitance changes with voltage between the different electron-contacts samples. The cells containing meso- $\mathrm{TiO}_{2}$ exhibit a sharp increase after $0.6 \mathrm{~V}$. Planar c- $\mathrm{TiO}_{2}$ cells show a similar evolution, although a negative capacitance region is perceived, whose onset is characterised by the downward spike, located around $100 \mathrm{~Hz}$. The negative capacitance, well documented in literature, is interpreted as the accumulation and subsequent discharge of both ionic and electronic carriers (holes) at the $\mathrm{TiO}_{2} /$ perovskite interface. ${ }^{26}$ Finally, ETL-free samples do not show the negative capacitance trend, but a continuous and exponential evolution of the low frequency capacitance with voltage, noted in Figure 3C. We can therefore conclude that low frequency capacitance at low and very high voltage show similar trends in the three different PSCs, but the intermediate voltages and the larger frequency region variations, indicate dynamic modifications in charge accumulation (ionic/electronic) that have significant consequences on the nature and timescales in a transient experiment, altering the hysteretic trends considerably. This observation correlates well with the different dynamic hysteresis responses observed previously.

To investigate the processes at the perovskite/ETL interface, we performed a dynamical optical investigation by means of transient absorption spectroscopy (TAS) in the nanosecond domain, as depicted in Figure 4. We monitored the TAS spectral evolution of the three different samples upon excitation with a light pulse at $600 \mathrm{~nm}$, ensuring a light penetration depth of less than $200 \mathrm{~nm} \cdot{ }^{27}$ Note that we keep the excitation density constant on the three samples, which have the same thickness, ensuring that a similar carrier density is generated. As reported in the Figure 4A-C, a negative band peaking at $760 \mathrm{~nm}$ appears, which is assigned to the photobleaching (PB), due to state filling of the conduction and valence band. Notably, the PB signal is proportional to the density of charges and its dynamical evolution reflect the charge dynamics. The PB band decreases in the time range investigated, as shown by the normalized dynamic at $760 \mathrm{~nm}$ probe wavelength presented in Figure 4D. It reveals a fast decay with a time constant of $t=100$ ns, accompanied by a longer component which does not decay in our temporal window $(1.5 \mu \mathrm{s})$ (a comparison of the decay in the 100ns range is reported in the inset of Figure 4B). Since we use an excitation density of around $50 \mathrm{~nJ} / \mathrm{cm}^{2}$ corresponding to a charge density of around $10^{17} \mathrm{~cm}^{-3}$, most of the traps are expected to be filled, thus the PB decay 
mainly mirrors the electron hole recombination. However, the PB signal persists on a longer timescale. As already observed by Leijtens T. et al, the long living component appearing in the TAS as well as in transient photocurrent measurements, can be related to trap-mediated recombination, i.e. trapped electrons or holes. ${ }^{28}$ Considering the same optical density for the three films and the same excitation density used, we can infer from the initial spectra (at 0-200 ns time window) that the photoinduced charge density at the compact- $\mathrm{TiO}_{2} /$ perovskite interface is slightly higher. However, for all cases, the long living component, sometimes also visible in photoluminescence measurements and still debated, can be related to the presence of long-living accumulated charges (possibly holes), ${ }^{28}$ located at the perovskite /ETL surface. Interestingly, the signal evolution (i.e. time constant) and magnitude of the long living component is similar in all cases, providing a clear indication that the interface charge dynamics in the ns-us time scale, relevant for the photovoltaic action in terms of charge collection, do not considerably differ comparing meso- $\mathrm{TiO}_{2}, \mathrm{c}-\mathrm{TiO}_{2}$ or FTO interfaces. On the other side, on the shorter time scale, the $\mathrm{PB}$ of the meso- $\mathrm{TiO}_{2}$ interface decays slower (a time constant of $50 \mathrm{~ns}$ is retrieved from fitting, as shown in Table 2), that might reflect a reduce electron hole recombination possibly due to reduced charge accumulation at this interface. This observation is in agreement with the slightly higher $\mathrm{V}_{\mathrm{oc}}$ that this sample presents over the others. A direct link with the device behaviour is rather challenging, however, the reduced charge accumulation at the meso- $\mathrm{TiO}_{2}$ interface might be a possible reason, among others, of the minimized dynamic hysteresis response for the mesoporous-based solar cell. Further work is ongoing to elucidate the interface dynamics and better establish a connection to the device scale.

We can therefore conclude that, while it is widely believed that the role of selective contacts towards photovoltage operates by controlling the built-in voltage, here we demonstrate that it rather depends on the internal recombination and splitting of Fermi levels inside the perovskite material. Our results indicate that the electric field is not the dominant charge extraction mechanism for the photocarriers, and very different electron contacts based on metal oxide/perovskite interface can behave similarly. Furthermore, the nature of the contact can drastically influence the dynamic response of charge accumulation at the interface, being an essential actor in controlling hysteresis and pretreatment dependence in time transient experiments.

\section{Experimental procedures}

\section{Solar cell fabrication}

Perovskite solar cells were fabricated on F-doped $\mathrm{SnO}_{2}$ (NSG10) substrates previously cleaned by a sequential sonication treatment in a $2 \%$ Hellmanex solution, acetone and isopropanol, followed by UV ozone treatment for $15 \mathrm{~min}$. These substrates were directly used as described for the ETL-free perovskite solar cells. To prepare the other configurations investigated in this manuscript, a compact blocking layer of $\mathrm{TiO}_{2}\left(\mathrm{c}-\mathrm{TiO}_{2}\right.$, 
$30 \mathrm{~nm}$ in thickness) was deposited by spray pyrolysis, using a titanium diisopropoxide bis(acetylacetonate) solution in ethanol $(22 \% \mathrm{v} / \mathrm{v})$, and then sinter at $450{ }^{\circ} \mathrm{C}$ for 20 minutes (c- $\mathrm{TiO}_{2}$ electrode). $\mathrm{SnO}_{2}$ electrodes were prepared by spin-coating a precursor solution of $\mathrm{SnCl}_{4}$ (Acros) dissolved in water. To form $\sim 100 \mathrm{~nm}$ thick $\mathrm{SnO}_{2}$ layer, $0.5 \mathrm{M}$ $\mathrm{SnCl}_{4}$ solution was spin-coated on the UV-ozone treated FTO substrates at 5,000 rpm for $10 \mathrm{~s}$. Then the $\mathrm{SnO}_{2}$ film was transferred onto a hotplate and dried at $100{ }^{\circ} \mathrm{C}$ for $1 \mathrm{~min}$, post-annealed at $180{ }^{\circ} \mathrm{C}$ for $1 \mathrm{~h}$ and cooled down before deposition of perovskite. The planar configurations, including the ETL-free cells, were treated by UV ozone for $15 \mathrm{~min}$ before deposition of perovskite layer. Finally, a $150 \mathrm{~nm}$ thick layer of mesoporous $\mathrm{TiO}_{2}$ (meso- $\mathrm{TiO}_{2}, 30$ NR-D titania paste from Dyesol) was prepared by spin-coating a diluted $\mathrm{TiO}_{2}$ dispersion in ethanol $\left(150 \mathrm{mg} \cdot \mathrm{ml}^{-1}\right)$, at $2000 \mathrm{rpm}$ for $15 \mathrm{~s}$ followed by a sintering step at $450{ }^{\circ} \mathrm{C}$ for $30 \mathrm{~min}$. Afterwards, the meso- $\mathrm{TiO}_{2}$ substrates were lithium-treated by spin-coating $40 \mu \mathrm{l}$ of tris(bis(trifluoromethylsulfonyl)imide) (Li-TFSI, 10mg/ml in acetonitrile) onto the mesoporous layer, followed by an additional sintering step at 450 ${ }^{\circ} \mathrm{C}$ for $20 \mathrm{~min}$. After sintering, the $\mathrm{c}-\mathrm{TiO}_{2} /$ meso- $\mathrm{TiO}_{2}$ electrodes were ready to use and transferred to a $\mathrm{N}_{2}$ controlled atmosphere. The perovskite precursor solution was prepared by mixing $\mathrm{CH}_{3} \mathrm{NH}_{3} \mathrm{I}$ (MAI, Dyesol) and $\mathrm{PbI}_{2}$ (TCI) in N,N'-dimethylsulfoxide (DMSO) in a molarity of 1.20 for $\mathrm{MAPbI}_{3}$, while for the mixed cation/halide composition formamidinium iodide (FAI), MAI (from Dyesol), $\mathrm{PbI}_{2}$ and $\mathrm{PbBr}_{2}$ (TCI) were mixed in DMF:DMSO (4:1) solvent at $1.25 \mathrm{M}$ to form $\left(\mathrm{FAPbI}_{3}\right)_{0.85}\left(\mathrm{MAPbBr}_{3}\right)_{0.15}$, with $5 \%$ of $\mathrm{PbI}_{2}$ excess content. The perovskite layers were then fabricated by using a two-steps spincoating process reported by Seok et al. ${ }^{14}$ (first step 1,000 r.p.m. for 10s; second step 4,000 r.p.m for $30 \mathrm{~s}$ ) and $10 \mathrm{~s}$ prior to the end of the program $100 \mu \mathrm{l}$ of chlorobenzene were poured onto the films. The substrates were then annealed at $100{ }^{\circ} \mathrm{C}$ during $60 \mathrm{~min}$. Afterwards, Spiro-OMeTAD was spin-coated at $4000 \mathrm{rpm}$ from a chlorobenzene solution (28.9mg in $400 \mu \mathrm{l}, 60 \mathrm{mmol})$ containing Li-TFSI $(7.0 \mu \mathrm{l}$ from a $520 \mathrm{mg} / \mathrm{ml}$ stock solution in acetonitrile), TBP $(11.5 \mu \mathrm{l})$ and Co(II)TFSI (10 mol \%, $8.8 \mu \mathrm{l}$ from a $40 \mathrm{mg} / \mathrm{ml}$ stock solution) as dopants. Finally, a $70 \mathrm{~nm}$ gold electrode was evaporated.

\section{Thin film characterization}

The XRD patterns of the prepared films were measured using a D8 Advance diffractometer from Bruker (Bragg-Brentano geometry, with an X-ray tube $\mathrm{Cu} \mathrm{K \alpha}$, $\lambda=1.5406 \AA$ ). The absorption spectra were registered with an UV-VIS-IR spectrophotometer (PerkinElmer Instrument). Photoelectron spectroscopy (PES) measurements were performed in a ultra-high vacuum analysis chamber (base pressure of $2 \times 10^{-10}$ mbar) using a He-discharge UV source (Omicron) with an excitation energy of $21.2 \mathrm{eV}$ for UPS. Before the analysis, the samples were treated by UV-ozone for 15 in the same conditions as for the device preparation. The photoelectron spectra were recorded using a Phoibos 100 (Specs) hemispherical energy analyzer at a pass energy of $5 \mathrm{eV}$ for the valence band. For work function determination, the secondary electron cut-off (SECO) was recorded by applying a $-10 \mathrm{~V}$ sample bias to clear the analyzer work function. The 
reported valence band spectra were background subtracted. The binding energies for all the photoemission spectra are referenced to the Fermi level. For the ns-Transient Absorption Spectroscopy (TAS)/Photoluminescence a ns laser ( $5 \mathrm{~ns}$ pulse duration, $10 \mathrm{~Hz}$, Ekspla NT342 model) with an integrated OPO system (from 355 to $2500 \mathrm{~nm}$ tunability) has been used as pump source. It is coupled with the LP980-KS Laser Flash Photolysis Spectrometer used for the measurement of laser induced transient absorption, and Photoluminescence kinetics and spectra. Wavelength specific kinetic measurements are made using photomultiplier and digital storage oscilloscope. The probe light is provided by a pulsed Xenon arc lamp. The beams are focused onto the sample on a diameter of minimum $5 \mathrm{~mm}^{2}$ area, ensuring the spatial overlap. The transmitted probe is spectrally filtered by a monochromator and detected. From the transmission change following photoexcitation the variation in the absorption is thus derived as $\Delta(\tau, \lambda)=\log \left(I_{\text {probe }}\right) /\left(I_{t(\tau, \lambda)}\right)$, where $\mathrm{I}_{\text {probe }}$ is the transmitted probe with excitation off and $\mathrm{I}_{t}$ is the transmitted probe after laser excitation. The minimum detectable optical density of the LP980-KS using the photomultiplier is $\Delta \mathrm{OD}=0.002$ (single shot, fast detector option) with a system overall response function of: $<7 \mathrm{~ns}$ (laser limited).

\section{Device characterization}

The photovoltaic device performance was analyzed using a VeraSol LED solar simulator (Newport) producing 1 sun AM $1.5\left(1000 \mathrm{~W} / \mathrm{m}^{2}\right)$ sunlight. Current-voltage curves were measured in air with a potentiostat (Keithley 2604). The light intensity was calibrated with a NREL certified KG5 filtered Si reference diode. The solar cells were masked with a metal aperture of $0.16 \mathrm{~cm}^{2}$ to define the active area. Current-voltage curves at different scan rates were collected from slowest to fastest scan rate by scanning in the FR direction followed by the RF direction for a given scan rate, with a time interval of 10 seconds (under illumination) before the next measurement. The starting voltage for the FR scan was slightly higher $(30 \mathrm{mV})$ than the $\mathrm{V}_{\mathrm{oc}}$ of the device while for the RF scan, it was $0 \mathrm{~V}$. EQE was measured with the IQE200B (Oriel) without bias light. Impedance spectroscopy measurements were performed in the dark for a cell area of $0.56 \mathrm{~cm}^{2}$. A perturbation amplitude of $10 \mathrm{mV}$ was used and the spectra measured over the frequency range $50 \mathrm{mHz}-1 \mathrm{MHz}$.

\section{Acknowledgements}

SR acknowledges Generalitat Valenciana for the grant GRISOLIA/2014/034. JB acknowledges funding from MINECO of Spain under Project MAT2016-76892-C3-1-R and Generalitat Valenciana Project PROMETEOII/2014/020. MKN acknowledge financial support European Commission H2020-ICT-2014-1, SOLEDLIGHT project, grant agreement N1: 643791 and the Swiss State Secretariat for Education, Research and Innovation (SERI), and CTI 15864.2 PFNM-NM, Solaronix, Aubonne, Switzerland. 


\section{Author contributions}

S.G. and C.R. fabricated the devices and carried out the majority of measurements. S.R. performed the impedance analysis. G.G. carried out the spectroscopic analysis. Y.L. prepared the $\mathrm{SnO}_{2}$ electrodes and mixed cation perovskite layers. M.R and N.K. performed the UPS characterization. C.R., J. B. and M. N. wrote the manuscript and interpreted the data. A. A. assisted in data analysis, and M.N. directed and supervised the project leading to this publication.

\section{Declaration of interest}

The authors declare no competing financial interest.

\section{Figure Captions}

Figure 1. Solar cell configurations, film characterization and photovoltaic performance of the perovskite cells. (A) Schematic diagram of the cell architectures. Compact and mesoposous- $\mathrm{TiO}_{2}\left(\right.$ meso- $\left.\mathrm{TiO}_{2}\right)$ layers are differing between configurations. (B) cross-sectional scanning electron microscopy (SEM) images obtained for each representative layout (bar-scale $1 \mu \mathrm{m}$ ). (C) Surface SEM images and optical properties of perovskite films analysed in (b). (D) X-ray diffraction (XRD) patterns of films analysed in (D).

Figure 2. $J-V$ performance and statistical analysis of perovskite solar cells. (A) Current-voltage $(J-V)$ curves measured under 1 sun illumination and (B) external quantum efficiency $(E Q E)$ of representative solar cells containing meso- $\mathrm{TiO}_{2}, \mathrm{c}-\mathrm{TiO}_{2}$ and ETL-free architectures ( $J_{\text {integrated }}$ is $21.2 \mathrm{~mA} \cdot \mathrm{cm}^{2}, 21.5 \mathrm{~mA} \cdot \mathrm{cm}^{2}$ and $21.3 \mathrm{~mA} \cdot \mathrm{cm}^{2}$ for). $(\mathbf{C})$ Summary of device parameters obtained for more than 40 cells measured under AM1.5G sun illumination, room temperature and air conditions. The work function of the different contact layers are also indicated for comparison (right panel).

Figure 3. Hysteresis behavior and impedance spectroscopy (IS) analysis. (A) $J-V$ scans for perovskite cells containing the three investigated ETL contacts (scan rates indicated in the legend); (B) absolute real values of the capacitance obtained from EIS measurements in the dark; (C) evolution of the low frequency capacitance versus applied voltage for the three investigated cells. The linear fit of $\ln C$ versus voltage for the ETLfree cell shows a slope of 5.62 .

Figure 4. Dynamical optical investigation using transient absorption spectroscopy (TAS). (A-C) TAS spectral evolution of the three different samples measured upon excitation from the glass side with an incident light pulse at $600 \mathrm{~nm}$ (penetration depth $\sim 200 \mathrm{~nm})^{27}$. (D) dynamics at 760nm revealing a faster time constant of $\mathrm{t}=100 \mathrm{~ns}$. 


\section{TABLES AND TABLE CAPTIONS}

Table 1. Device parameters obtained from the cell configurations shown in Figure

2. The average values obtained for each device configuration are included in brackets.

\begin{tabular}{|c|l|l|l|l|}
\hline Cell & \multicolumn{1}{|c|}{$\begin{array}{c}\boldsymbol{J}_{\text {sc }}(\mathbf{m A} \\
\left.\mathbf{c m}^{\mathbf{2}}\right)\end{array}$} & $\boldsymbol{V}_{\boldsymbol{~ o c}}(\mathbf{V})$ & $\boldsymbol{F F}$ & $\boldsymbol{P C E}(\boldsymbol{\%})$ \\
\hline $\mathrm{Meso}-\mathrm{TiO}_{2}$ & $21.91(21.9 \pm 0.8)$ & $1.09(1.078 \pm 0.007)$ & $0.78(0.77 \pm 0.02)$ & $18.75(18.17 \pm 0.8)$ \\
\hline$c-\mathrm{TiO}_{2}$ & $21.7(21.2 \pm 0.9)$ & $1.07(1.02 \pm 0.03)$ & $0.70(0.67 \pm 0.05)$ & $16.25(15 \pm 1)$ \\
\hline $\mathrm{FTO}$ & $21.08(21.1 \pm 0.6)$ & $1.04(0.98 \pm 0.08)$ & $0.64(0.62 \pm 0.03)$ & $14.03(14 \pm 1)$ \\
\hline
\end{tabular}

Table 2. Optical parameters obtained from the TAS analysis.

\begin{tabular}{|c|c|c|}
\hline Sample & $(\mathrm{ns})^{\mathbf{t 1}}$ & $\begin{array}{r}\mathbf{t 2} \\
(\mathrm{ns})\end{array}$ \\
\hline FTO/MAPbI $_{3}$ & 21 & 98 \\
\hline FTO/c-TiO$_{2} / \mathrm{MAPbI}_{3}$ & 13 & 98 \\
\hline 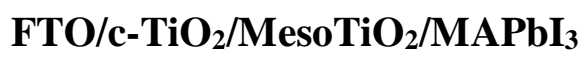 & 50 & 269 \\
\hline
\end{tabular}


Figures

A

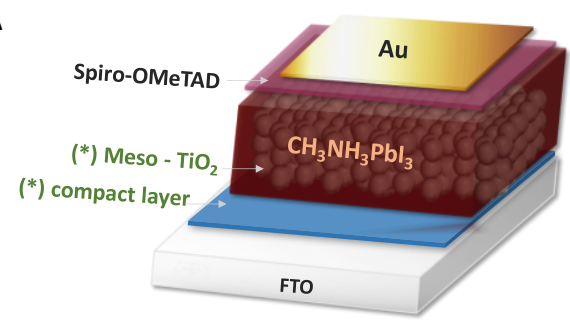

(*) Not present in all configurations
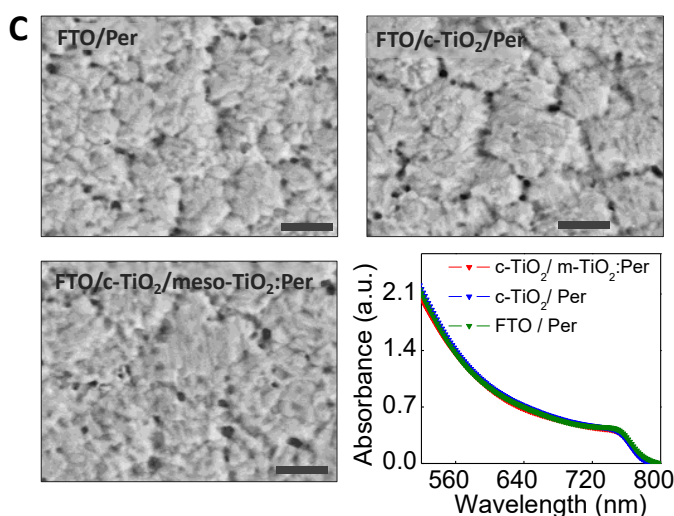

B
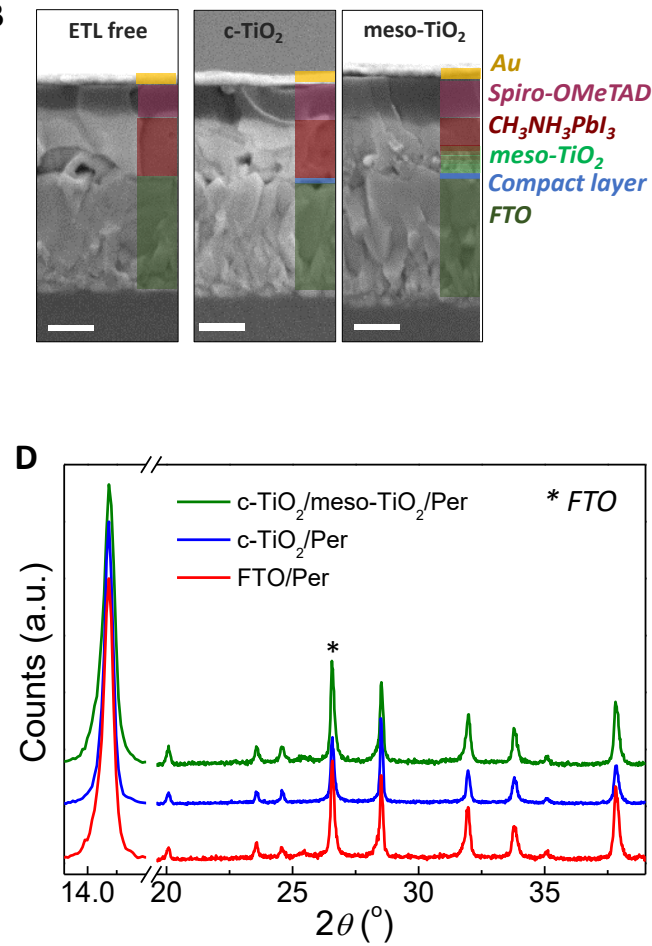

Figure 1. 

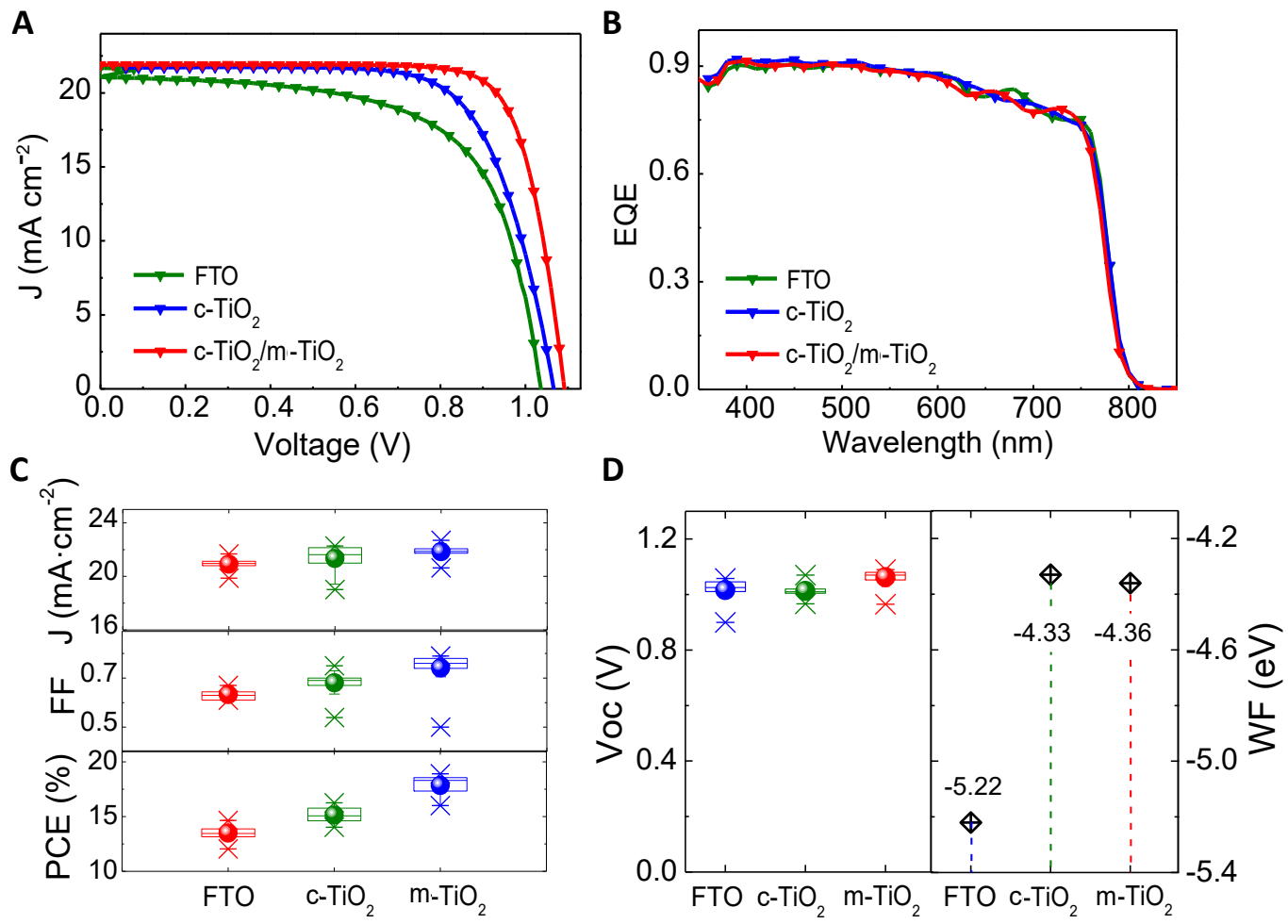

Figure 2. 

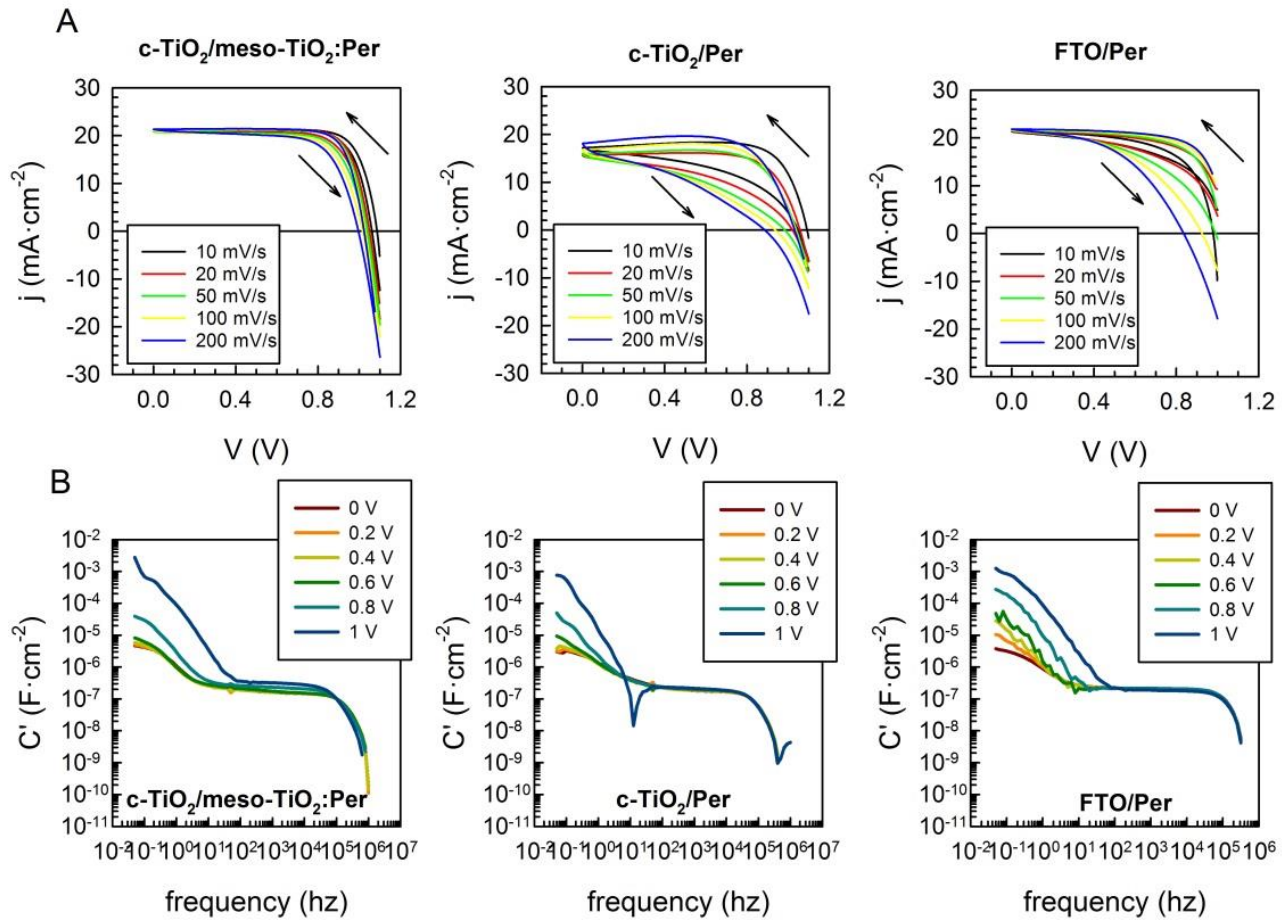

C
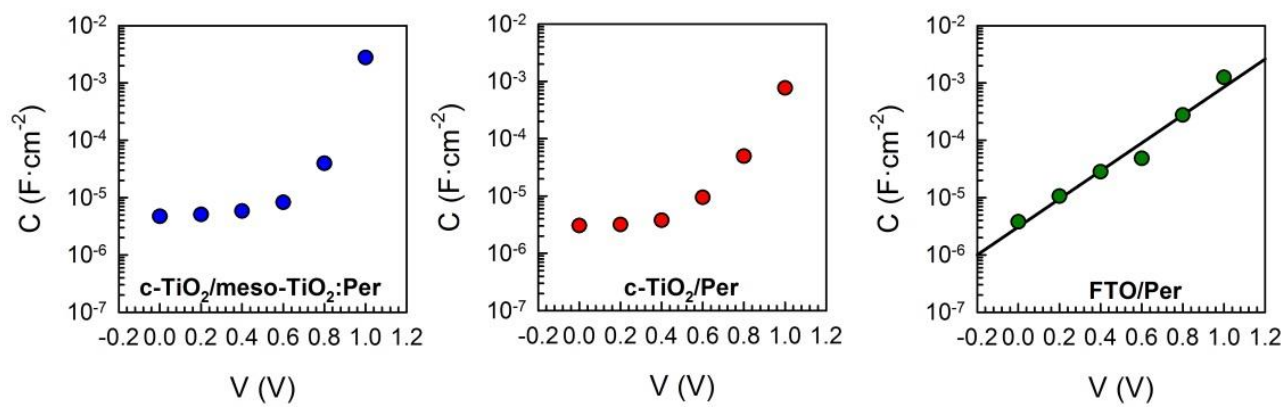

Figure 3. 

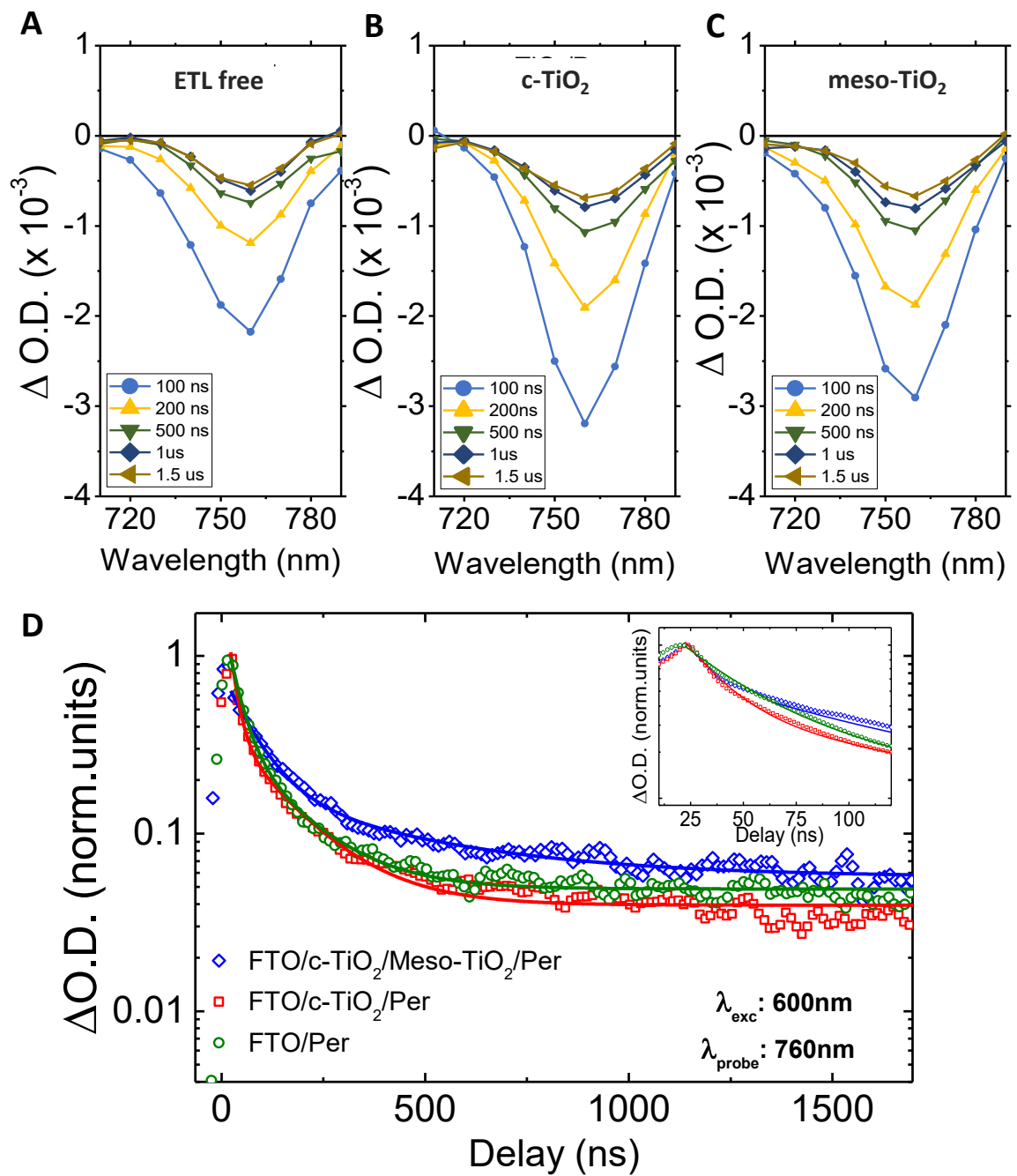

Figure 4. 


\section{References}

1. Etgar, L.; Gao, P.; Xue, Z.; Peng, Q.; Chandiran, A. K.; Liu, B.; Nazeeruddin, M. K.; Grätzel, M. Mesoscopic $\mathrm{CH}_{3} \mathrm{NH}_{3} \mathrm{PbI}_{3} / \mathrm{TiO}_{2}$ heterojunction solar cells. Journal of the American Chemical Society 2012, 134 (42), 17396-17399.

2. Ku, Z.; Rong, Y.; Xu, M.; Liu, T.; Han, H. Full Printable Processed Mesoscopic $\mathrm{CH}_{3} \mathrm{NH}_{3} \mathrm{PbI}_{3} / \mathrm{TiO}_{2}$ Heterojunction Solar Cells with Carbon Counter Electrode. Scientific Reports 2013, 3, 3132.

3. Mei, A.; Li, X.; Liu, L.; Ku, Z.; Liu, T.; Rong, Y.; Xu, M.; Hu, M.; Chen, J.; Yang, Y.; Grätzel, M.; Han, H. A hole-conductor-free, fully printable mesoscopic perovskite solar cell with high stability. Science 2014, 345 (6194), 295-298.

4. Shin, S. S.; Yeom, E. J.; Yang, W. S.; Hur, S.; Kim, M. G.; Im, J.; Seo, J.; Noh, J. H.; Seok, S. I. Colloidally prepared La-doped $\mathrm{BaSnO}_{3}$ electrodes for efficient, photostable perovskite solar cells. Science 2017, 356 (6334), 167-171.

5. Xiao, Z.; Kerner, R. A.; Zhao, L.; Tran, N. L.; Lee, K. M.; Koh, T.-W.; Scholes, G. D.; Rand, B. P. Efficient perovskite light-emitting diodes featuring nanometre-sized crystallites. Nature Photonics 2017, 11 (2), 108-115.

6. Fang, Y.; Dong, Q.; Shao, Y.; Yuan, Y.; Huang, J. Highly narrowband perovskite single-crystal photodetectors enabled by surface-charge recombination. Nature Photonics 2015, 9 (10), 679.

7. Senanayak, S. P.; Yang, B.; Thomas, T. H.; Giesbrecht, N.; Huang, W.; Gann, E.; Nair, B.; Goedel, K.; Guha, S.; Moya, X. Understanding charge transport in lead iodide perovskite thin-film field-effect transistors. Science Advances 2017, 3 (1), e1601935.

8. Xu, J.; Chen, Y.; Dai, L. Efficiently photo-charging lithium-ion battery by perovskite solar cell. Nature communications 2015, 6, 8103.

9. Xing, G.; Mathews, N.; Sun, S.; Lim, S. S.; Lam, Y. M.; Grätzel, M.; Mhaisalkar, S.; Sum, T. C. Long-Range Balanced Electron- and Hole-Transport Lengths in OrganicInorganic $\mathrm{CH}_{3} \mathrm{NH}_{3} \mathrm{PbI}_{3}$. Science 2013, 342 (6156), 344-347.

10. Zhu, H.; Fu, Y.; Meng, F.; Wu, X.; Gong, Z.; Ding, Q.; Gustafsson, M. V.; Trinh, M. T.; Jin, S.; Zhu, X. Lead halide perovskite nanowire lasers with low lasing thresholds and high quality factors. Nature materials 2015, 14 (6), 636.

11. Kagan, C.; Mitzi, D.; Dimitrakopoulos, C. Organic-inorganic hybrid materials as semiconducting channels in thin-film field-effect transistors. Science 1999, 286 (5441), 945-947.

12. Tress, W.; Marinova, N.; Moehl, T.; Zakeeruddin, S. M.; Nazeeruddin, M. K.; Gratzel, M. Understanding the rate-dependent J-V hysteresis, slow time component, and aging in $\mathrm{CH}_{3} \mathrm{NH}_{3} \mathrm{PbI}_{3}$ perovskite solar cells: the role of a compensated electric field. Energy \& Environmental Science 2015, 8 (3), 995-1004. 
13. Richardson, G.; O'Kane, S. E.; Niemann, R. G.; Peltola, T. A.; Foster, J. M.; Cameron, P. J.; Walker, A. B. Can slow-moving ions explain hysteresis in the currentvoltage curves of perovskite solar cells? Energy \& Environmental Science 2016, 9 (4), 1476-1485.

14. Jeon, N. J.; Noh, J. H.; Yang, W. S.; Kim, Y. C.; Ryu, S.; Seo, J.; Seok, S. I. Compositional engineering of perovskite materials for high-performance solar cells. Nature 2015, 517 (7535), 476-480.

15. Gottesman, R.; Lopez-Varo, P.; Gouda, L.; Jimenez-Tejada, J. A.; Hu, J.; Tirosh, S.; Zaban, A.; Bisquert, J. Dynamic Phenomena at Perovskite/Electron-Selective Contact Interface as Interpreted from Photovoltage Decays. Chem 2016, 1 (5), 776-789.

16. Rong, Y.; Hu, Y.; Ravishankar, S.; Liu, H.; Hou, X.; Sheng, Y.; Mei, A.; Wang, Q.; Li, D.; Xu, M.; Bisquert, J.; Han, H. Tunable hysteresis effect for perovskite solar cells. Energy \& Environmental Science 2017.

17. Rong, Y.; Tang, Z.; Zhao, Y.; Zhong, X.; Venkatesan, S.; Graham, H.; Patton, M.; Jing, Y.; Guloy, A. M.; Yao, Y. Solvent engineering towards controlled grain growth in perovskite planar heterojunction solar cells. Nanoscale 2015, 7 (24), 10595-10599.

18. Juarez-Perez, E. J.; Wußler, M.; Fabregat-Santiago, F.; Lakus-Wollny, K.; Mankel, E.; Mayer, T.; Jaegermann, W.; Mora-Sero, I. Role of the selective contacts in the performance of lead halide perovskite solar cells. The Journal of Physical Chemistry Letters 2014, 5 (4), 680-685.

19. Kim, H.-S.; Park, N.-G. Parameters affecting I-V hysteresis of $\mathrm{CH}_{3} \mathrm{NH}_{3} \mathrm{PbI}_{3}$ perovskite solar cells: effects of perovskite crystal size and mesoporous $\mathrm{TiO} 2$ layer. The Journal of Physical Chemistry Letters 2014, 5 (17), 2927-2934.

20. Sanchez, R. S.; Gonzalez-Pedro, V.; Lee, J.-W.; Park, N.-G.; Kang, Y. S.; MoraSero, I.; Bisquert, J. Slow dynamic processes in lead halide perovskite solar cells. Characteristic times and hysteresis. The Journal of Physical Chemistry Letters 2014, 5 (13), 2357-2363.

21. Bisquert, J. The Physics of Solar Cells: Perovskites, Organics, and Photovoltaic Fundamentals. CRC Press: 2017.

22. Würfel, U.; Cuevas, A.; Würfel, P. Charge carrier separation in solar cells. IEEE Journal of Photovoltaics 2015, 5 (1), 461-469.

23. Bergmann, V. W.; Guo, Y.; Tanaka, H.; Hermes, I. M.; Li, D.; Klasen, A.; Bretschneider, S. A.; Nakamura, E.; Berger, R. d.; Weber, S. A. Local time-dependent charging in a perovskite solar cell. ACS Applied Materials \& Interfaces 2016, 8 (30), 19402-19409.

24. Garcia-Belmonte, G.; Bisquert, J. Distinction between Capacitive and Noncapacitive Hysteretic Currents in Operation and Degradation of Perovskite Solar 
Cells. ACS Energy Letters 2016, 1 (4), 683-688.

25. Zarazua, I.; Bisquert, J.; Garcia-Belmonte, G. Light-induced space-charge accumulation zone as photovoltaic mechanism in perovskite solar cells. The Journal of Physical Chemistry Letters 2016, 7 (3), 525-528.

26. Ghahremanirad, E.; Bou, A.; Olyaee, S.; Bisquert, J. Inductive Loop in the Impedance Response of Perovskite Solar Cells Explained by Surface Polarization Model. The Journal of Physical Chemistry Letters 2017, 1402-1406.

27. Berera, R.; van Grondelle, R.; Kennis, J. T. Ultrafast transient absorption spectroscopy: principles and application to photosynthetic systems. Photosynthesis Research 2009, 101 (2-3), 105-118.

28. Li, W.; Liu, J.; Bai, F.-Q.; Zhang, H.-X.; Prezhdo, O. V. Hole Trapping by Iodine Interstitial Defects Decreases Free Carrier Losses in Perovskite Solar Cells: A TimeDomain Ab Initio Study. ACS Energy Letters 2017, 2, 1270-1278. 
Supporting Information for

\section{Influence of charge transport layers on open-circuit voltage and hysteresis in Perovskite Solar Cells}

Sandheep Ravishankar, Saba Gharibzadeh, Cristina Roldán-Carmona, Giulia Grancini, Yonghui Lee, Maryline Ralaiarisoa, Abdullah M. Asiri, Nobert Koch, Juan Bisquert and Mohammad Khaja Nazeeruddin 


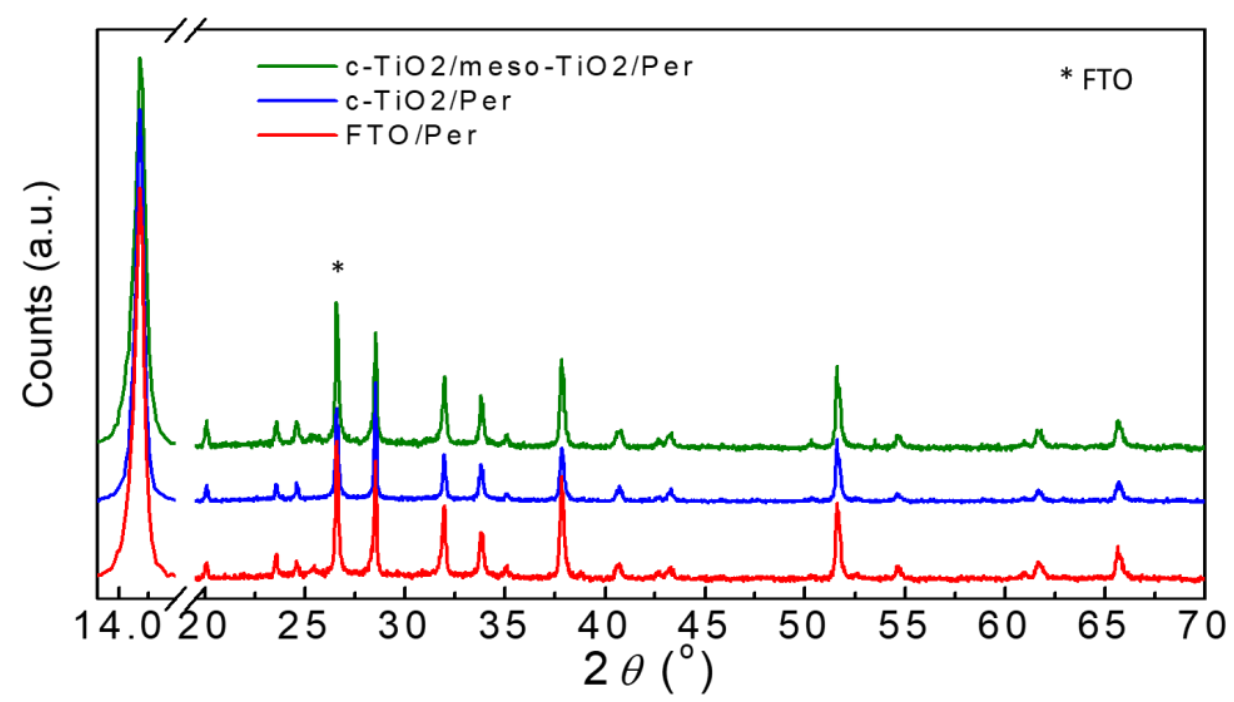

Figure S1. X-Ray Diffraction (XRD) pattern of perovskite films. Diffractogram patterns corresponding to perovskite films grown on different ETL contacts.
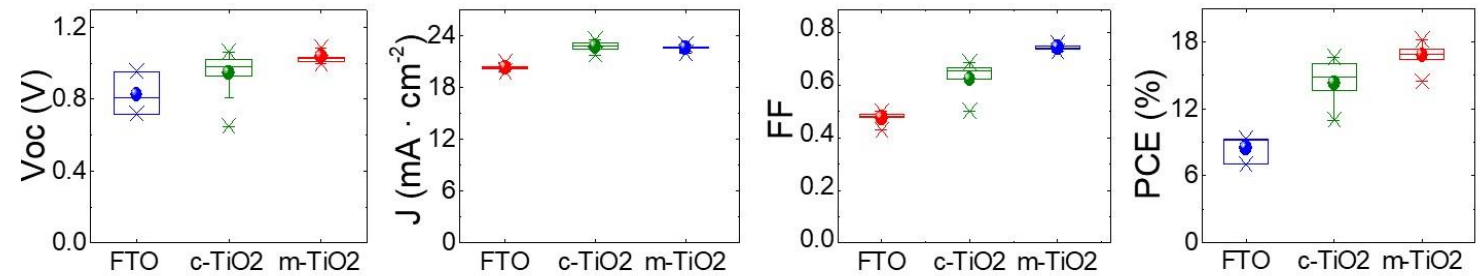

Figure S2. Statistical analysis of mixed cation/mixed halide perovskite solar cells. Device parameters obtained from 30 cells measured under AM1.5G sun illumination, room temperature and air conditions.

Table S1. Statistical analysis of mixed cation/mixed halide perovskite solar cells. Device parameters obtained from cells shown in Figure S2.

\begin{tabular}{|l|c|c|c|c|}
\hline Contact layer & $\boldsymbol{P C E}(\%)$ & $J_{\text {sc }}\left(\boldsymbol{m} \boldsymbol{A} \mathbf{c m}^{-2}\right)$ & $\boldsymbol{F F}$ & $V_{\text {oc }}(\boldsymbol{V})$ \\
\hline Meso-TiO $_{2}$ & $16.83 \pm 1.14$ & $22.12 \pm 0.9$ & $0.73 \pm 0.03$ & $1.04 \pm 0.03$ \\
\hline Compact-TiO $_{2}$ & $13.8 \pm 2.5$ & $22.7 \pm 0.5$ & $0.62 \pm 0.07$ & $0.98 \pm 0.07$ \\
\hline FTO & $7.3 \pm 1.5$ & $20.5 \pm 0.6$ & $0.48 \pm 0.03$ & $0.83 \pm 0.1$ \\
\hline
\end{tabular}




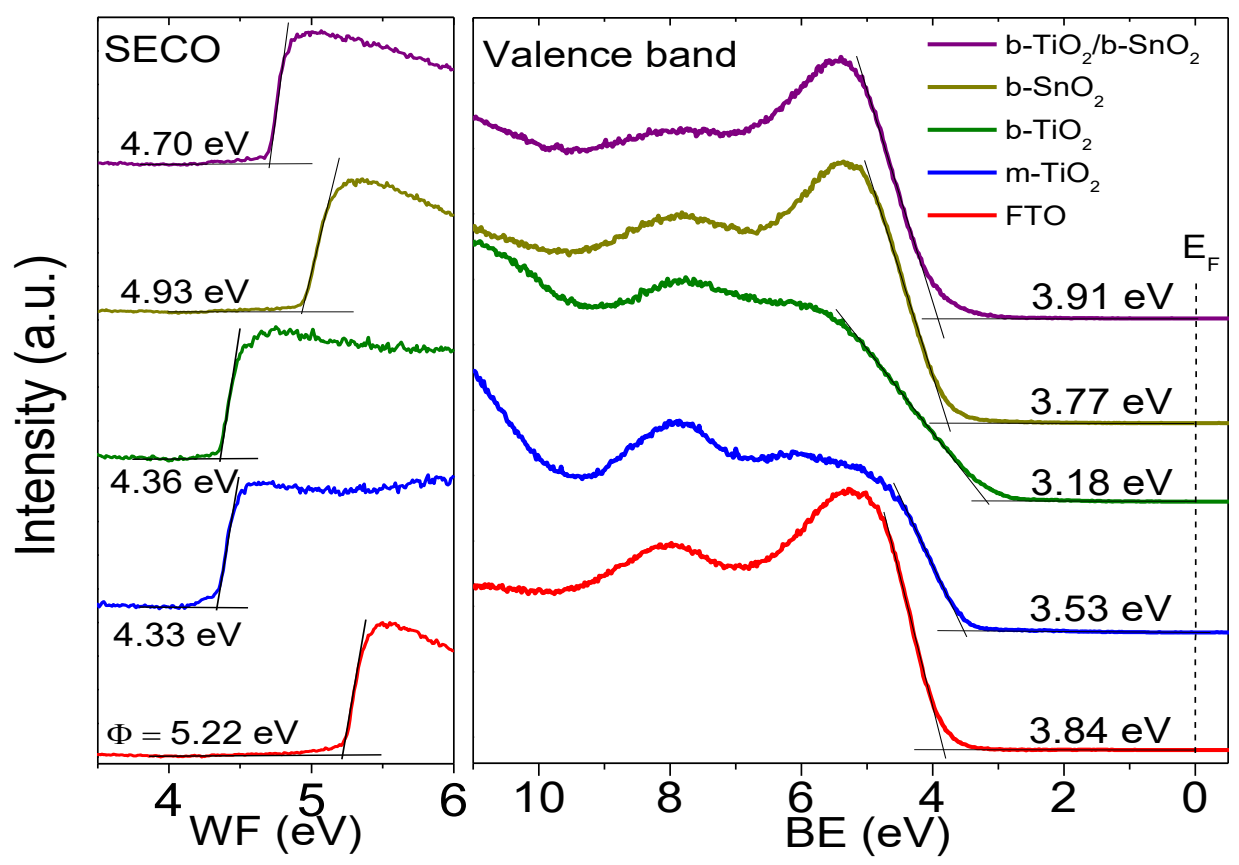

Figure S3. UPS characterization. Secondary-electron cutoffs for work function $(\Phi)$ determination (left), and binding energy range valence spectra (right) of the different electrodes. The work function values in $\mathrm{eV}$ is indicated in the inset. 
A

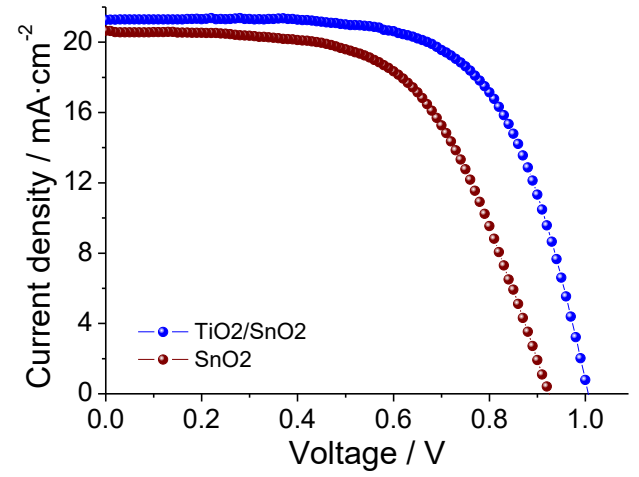

C

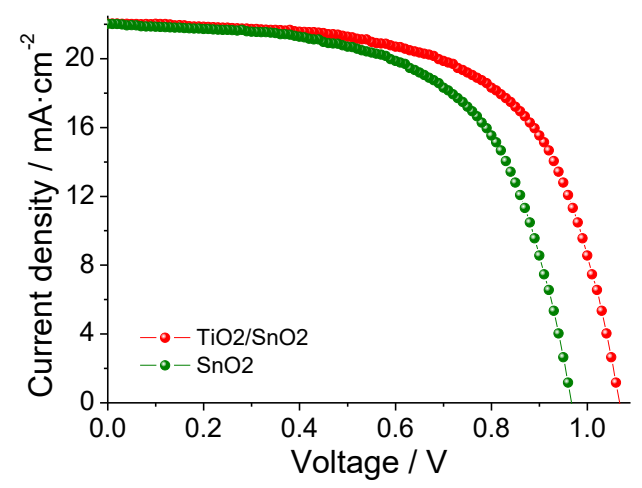

B
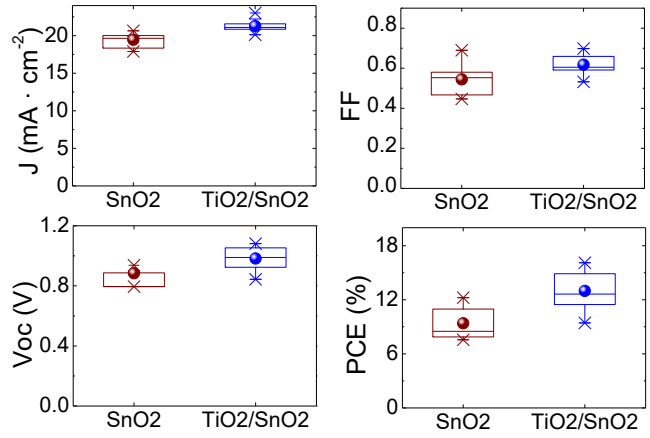

D
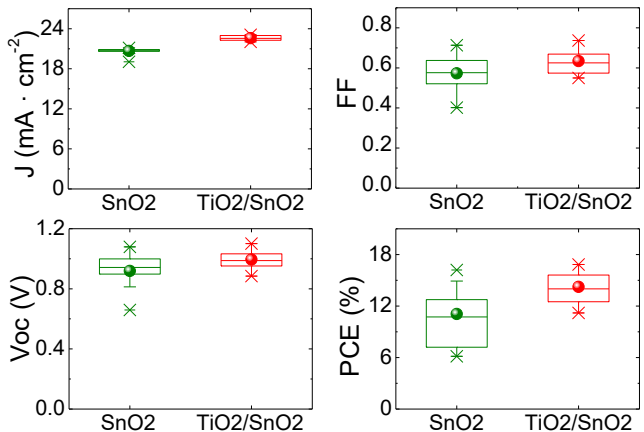

Figure $\mathrm{S4}$. $\mathrm{SnO}_{2}$ based contacts. Current-voltage $(J-V)$ curves and summary of device parameters obtained from: (A, B) 22 devices based on $\mathrm{MAPbI}_{3}$ perovskite and $(\mathbf{C}, \mathbf{D}) 29$ devices based on mixed $\left(\mathrm{FAPbI}_{3}\right)_{0.85}\left(\mathrm{MAPbBr}_{3}\right)_{0.15}$ perovskite, measured under AM1.5G sun illumination, room temperature and air conditions. 


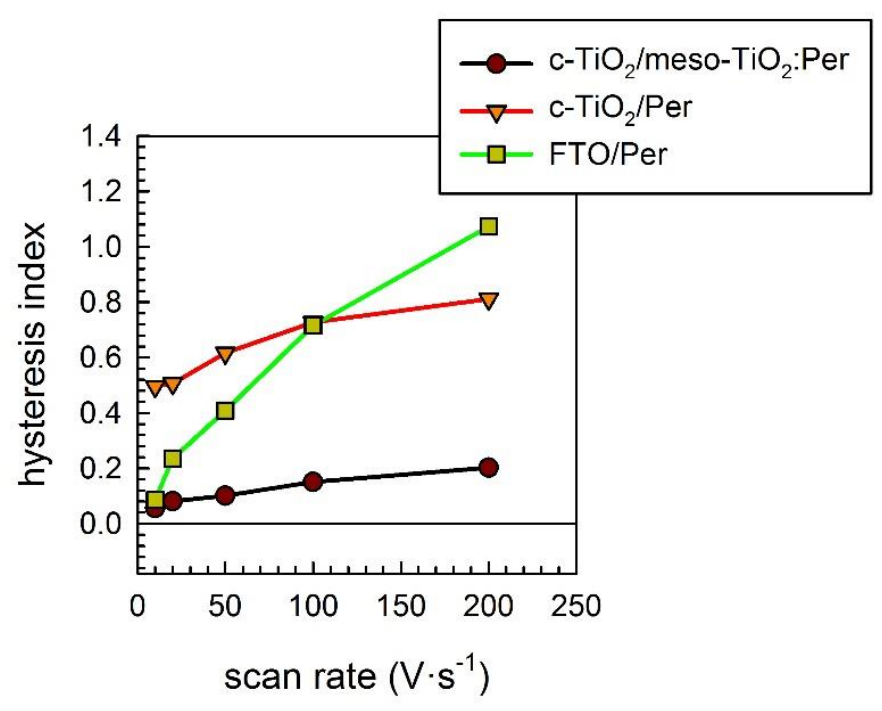

Figure S5. Hysteresis index analysis. Calculated hysteresis index at maximum power point versus scan rate for devices containing meso- $\mathrm{TiO}_{2}, \mathrm{c}-\mathrm{TiO}_{2}$ as ETL layers, as well as ETL-free cells. 


\section{Chapter 7}

\section{Publication 4}

Effects of Frequency Dependence of the External Quantum Efficiency of Perovskite Solar Cells

Ravishankar, S.; Aranda, C.; Boix, P. P.; Anta, J. A.; Bisquert, J.; Garcia-Belmonte, G. Effects of Frequency Dependence of the External Quantum Efficiency of Perovskite Solar Cells. The Journal of Physical Chemistry Letters 2018, 9 (11), 3099-3104. Reprinted with permission, Copyright 2018, American Chemical Society.

\section{Candidate's contribution}

\begin{tabular}{|c|c|}
\hline Nature of Contribution & Extent of Contribution \\
\hline $\begin{array}{l}\text { - Carried out all the measurements } \\
\text { - Developed the model and carried } \\
\text { out the simulations } \\
\text { - Wrote first draft of the manuscript } \\
\text { - Wrote first draft of the reply to } \\
\text { referees }\end{array}$ & $80 \%$ \\
\hline
\end{tabular}

\section{Introduction}

After establishing the role of the external contacts on the obtained $V_{o c}$ in the previous chapter, the effect of the contacts, if any, on the photocurrent extraction was investigated. This chapter identifies the role of the LF capacitance and resistance in reducing the EQE of the PSC at low frequencies due to an internal displacement of the photovoltage.

\section{Published manuscript}




\title{
Effects of Frequency Dependence of the External Quantum Efficiency of Perovskite Solar Cells
}

\author{
Sandheep Ravishankar, ${ }^{1 *}$ Clara Aranda, ${ }^{1}$ Pablo. P. Boix, ${ }^{2}$ Juan A. Anta, ${ }^{3}$ Juan \\ Bisquert $^{* 1}$ and Germà Garcia-Belmonte ${ }^{1 *}$
}

\author{
${ }^{1}$ Institute of Advanced Materials (INAM), Universitat Jaume I, 12006 Castelló, Spain \\ ${ }^{2}$ Instituto de Ciencia Molecular (ICMol), Universitat de València, Catedrático José \\ Beltrán, 2, 46980 Paterna, Spain \\ ${ }^{3}$ Department of Physical, Chemical and Natural Systems, University Pablo de Olavide, \\ 41013, Sevilla, Spain
}

Email: ravishan@uji.es, bisquert@uji.es,garciag@uji.es

\begin{abstract}
Perovskite solar cells are known to show very long response timescales, in the order of milliseconds to seconds. This generates considerable doubt over the validity of the measured external quantum efficiency and consequently the estimation of the short circuit current density. We observe a variation as high as $10 \%$ in the values of the EQE of perovskite solar cells for different optical chopper frequencies between $10-500 \mathrm{~Hz}$, indicating a need to establish well-defined protocols of external quantum efficiency measurement. We also corroborate these values and obtain new insights regarding the working mechanisms of perovskite solar cells from intensity modulated photocurrent spectroscopy measurements, identifying the evolution of the external quantum efficiency over a range of frequencies, displaying a singular reduction at very low frequencies. This reduction in external quantum efficiency is ascribed to additional resistive contributions hindering charge extraction in the perovskite solar cell at short circuit conditions, which are delayed because of the concomitant large low-frequency capacitance.
\end{abstract}

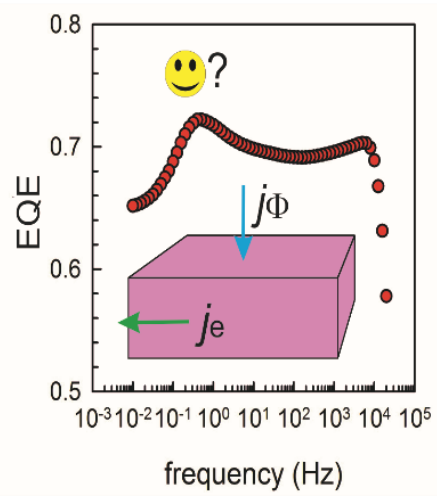


The external quantum efficiency (EQE) is a central characterization tool to estimate the performance of a photovoltaic cell. It is defined as the ratio of number of collected electron-hole pairs to the number of photons impinging on the device. ${ }^{1}$ The EQE provides information on how efficiently the solar cell is able to absorb a photon, generate an electron-hole pair, separate and subsequently extract these carriers at respective selective contacts. It can also provide device parameters such as the short-circuit current $\left(j_{s c}\right)$ and in some cases even the diffusion length, depending upon the dominant transport mechanism. An example being the diffusion-recombination model for dye sensitized solar cells ${ }^{2}$ which allows discerning transport from recombination time. Traditionally, the EQE is measured at short circuit (SC) by applying a monochromatic AC small perturbation in light intensity and measuring the response of the solar cell, subsequently scanned over the range of wavelengths of incoming light. The solar cell is kept under a DC biasing white light intensity that can range from $5-100 \mathrm{~mW} \cdot \mathrm{cm}^{-2}$ during the measurement, ${ }^{3}$ although in most cases of collection efficiency measurements, the bias intensity is very low, between $5-10 \mathrm{~mW} \cdot \mathrm{cm}^{-2},{ }^{4-5}$ whose main purpose is to provide a small background charge density to raise the conductivity of the solar cell from its minimal dark value.

The modulation frequency of the AC small perturbation in light intensity is determined by an optical chopper ${ }^{6}$ and is assumed to be much lower (a few hundred $\mathrm{Hz}$ ) ${ }^{7-9}$ than the frequency associated with the processes of carrier generation, transport, recombination (lifetime) and charge storage mechanisms, and hence should not have an influence on the measured EQE. However, the validity of this assumption is not assured in the case of perovskite solar cells (PSCs). PSCs are known to show responses at very long timescales in the order of milliseconds to seconds, observed in both voltage and current evolution, which has been confirmed from a wide variety of experiments such as open-circuit voltage $\left(V_{o c}\right)$ decay, ${ }^{10}$ stepped voltage scans ${ }^{11}$ and current decay from photoinduced charge extraction (PI-CE) measurements. ${ }^{12}$ This has also been observed in the EQE, which has been seen to evolve over timescales of minutes in both dark and light conditions, ${ }^{11,13}$ making the estimation of the $j_{s c}$ very difficult. ${ }^{14}$ Furthermore, a large capacitance of the order of $\mathrm{mF} \mathrm{cm}^{-2}$ has been observed from impedance spectroscopy (IS) measurements at $\mathrm{SC},{ }^{15}$ similar in magnitude to that observed at open-circuit (OC) conditions, ${ }^{16}$ which is likely related to the aforementioned EQE evolution. Therefore, a clarification regarding the nature of EQE evolution in perovskites and its contributing factors is required to better understand its operation and limiting mechanisms at SC and ascertain the validity of the EQE obtained at a given state of the perovskite solar cell.

We first provide a general definition of the EQE as obtained from standard experimental practices. Let $j_{e}$ be the extracted photocurrent density while the maximal current density $j_{\Phi}=q \Phi$ is defined from $\Phi$, the input photon flux at a given wavelength $\lambda$, and $q$ the elementary charge. The steady state EQE, EQEPV-ss, is

$$
E Q E_{P V-S S}=\frac{\overline{j_{e}}}{\overline{j_{\Phi}}}
$$

The overhead bars indicate conditions of steady state. A traditional experimental 
method termed the differential spectral response method, EQEPV-DIFF, involves the measurement of the photocurrent at SC upon application of a small low frequency AC perturbation of monochromatic light (using an optical chopper) to the sample, which is illuminated by a DC bias white-light intensity. Under an applied small perturbation of light intensity $j_{\Phi}=\overline{j_{\Phi}}+\tilde{j}_{\Phi}$, we can write $j_{e}=\overline{j_{e}}+\tilde{j}_{e}$,

$$
E Q E_{P V-D I F F}=\frac{\tilde{j}_{e}}{\tilde{j}_{\Phi}} \approx \frac{d \overline{j_{e}}}{d \overline{j_{\Phi}}}
$$

Equation 2 is an approximation because it assumes an infinitely slow (zero frequency) perturbation, while realistic measurements involve frequencies in the range of a few hundred $\mathrm{Hz}$ imparted by the optical chopper.

For the sake of generality, a power-law relationship can describe the most common dependences of collected current on photon flux as:

$$
\overline{j_{e}}(\lambda)=k{\overline{j_{\Phi}}}^{n}(\lambda)
$$

where $n$ accounts for a power-law exponent and $k$ is a proportionality constant. This is because we are normally interested in the variation of EQE over several decades of photon flux from which $n$ is readily derived. Thus we have

$$
E Q E_{P V-D I F F}=n k{\overline{j_{\Phi}}}^{n-1}
$$

We also corroborate the EQEPV-DIFF values with IMPS measurements, which involve the measurement of modulated extracted photocurrent versus modulated input photon current density. The transfer function of IMPS is given by ${ }^{17}$

$$
Q(\omega)=\frac{\tilde{J}_{e}(\omega)}{\tilde{J}_{\Phi}(\omega)}
$$

Because of the modulated character of both input and output signals, one can expect inphase and out-of-phase components of the transfer function, similarly to that occurring when measuring impedance spectroscopy. This implies the complex (real and imaginary) form of the IMPS measurement so that $Q=Q Q^{\prime}+i Q^{\prime}$, whose low-frequency limit is purely real and it is given by

$$
Q(0)=\frac{d \overline{j_{e}}}{d \overline{j_{\Phi}}}
$$

which corresponds to the definition in Equation 2 provided that $\omega=0$. Therefore, the $\mathrm{EQE}_{\mathrm{PV}-\mathrm{DIFF}}$ value ideally approximates the low frequency limit of the IMPS transfer function. We note that the measurement of EQE (differential spectral response method) and from IMPS measurements are identical in the sense that they are both small perturbation methods, the only difference being that the AC perturbation in direct measurements is generated using an optical chopper with the incident light beam, whereas the AC light perturbation in IMPS is generated by driving an AC current through the light source using a potentiostat. In both cases, it is ensured that the light perturbation acting on the solar cells is the same in the full range of light 
intensities and frequencies. The other difference is in their application, since IMPS is usually considered as a frequency-resolved method to obtain kinetic information. However, from the point of view of EQE analysis, we can use both approaches in combination.

The above mentioned definitions are summarized in Table 1. By comparing Equations 1 and 4, we can see that the values of EQE obtained from small-perturbation methods and steady-state methods converge only if the relationship between extracted photocurrent and input photon flux is linear.

Table 1 Definition of external quantum efficiency according to the measurement procedure. In the differential spectral response method, the output is a function of the optical chopper frequency, as indicated.

\begin{tabular}{|l|l|c|}
\hline EQEPV-SS & Steady state measurement & $\frac{\overline{j_{e}}}{\overline{j_{\Phi}}}$ \\
\hline EQEPV-DIFF & $\begin{array}{l}\text { Differential spectral response } \\
\text { method }\end{array}$ & $\frac{d j_{e}}{d j_{\Phi}}\left(\omega_{\text {chopper }}\right)$ \\
\hline IMPS & $\begin{array}{l}\text { Intensity modulated photocurrent } \\
\text { spectroscopy transfer function } Q\end{array}$ & $\frac{\tilde{j}_{e}(\omega)}{\widetilde{j}_{\Phi}(\omega)}$ \\
\hline
\end{tabular}

We now proceed to identify the trends of EQE evolution with input photon current density in perovskite solar cells and to clarify the above described methods of EQE measurement. Figure SI1a and SI1b show the $J-V$ curve of the perovskite solar cell tested and its corresponding maximum photocurrent. In addition to measuring EQEPV-DIFF directly, we also obtain the value indirectly using the following methods. The first method involves determination of EQEPV-ss from steady state measurements of extracted photocurrent density for a given input photon current density. We then obtain EQEPV-DIFF from the slope of the data obtained from the steady state measurements, as shown in Equation 2. The second method involves measurement of IMPS spectra and extracting the EQE $E_{P V-D I F F}$ value, which corresponds to the low-frequency limit of the transfer function, as shown in Equation 6. Both EQEPV-ss and IMPS measurements were carried out using a blue light $(470 \mathrm{~nm})$ source in order to avoid effects relating to absorption losses (see Figure SI2 for absorption spectrum). 
(a)

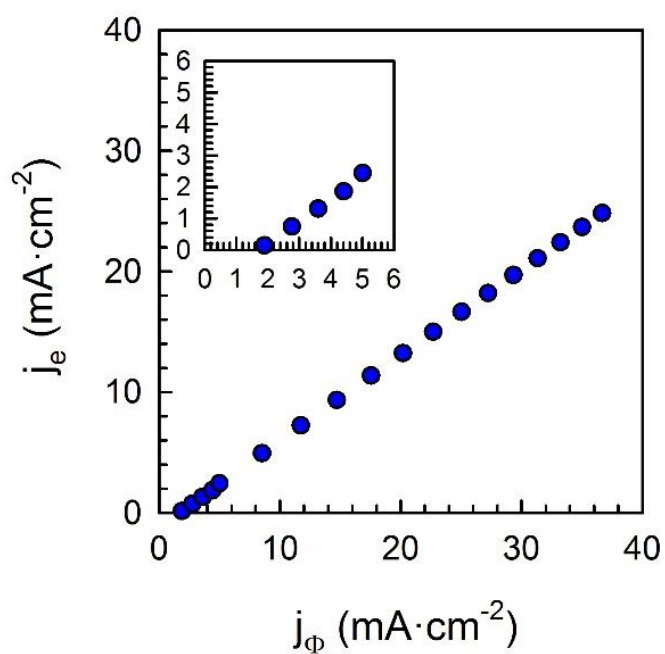

(c)

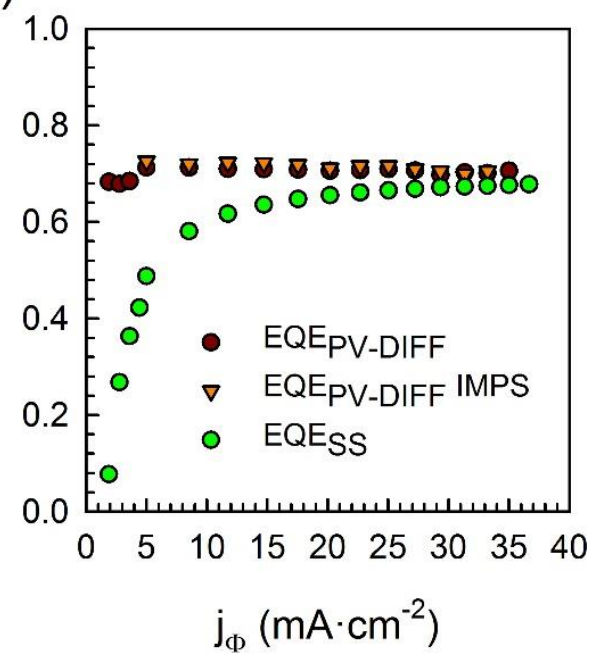

(b)

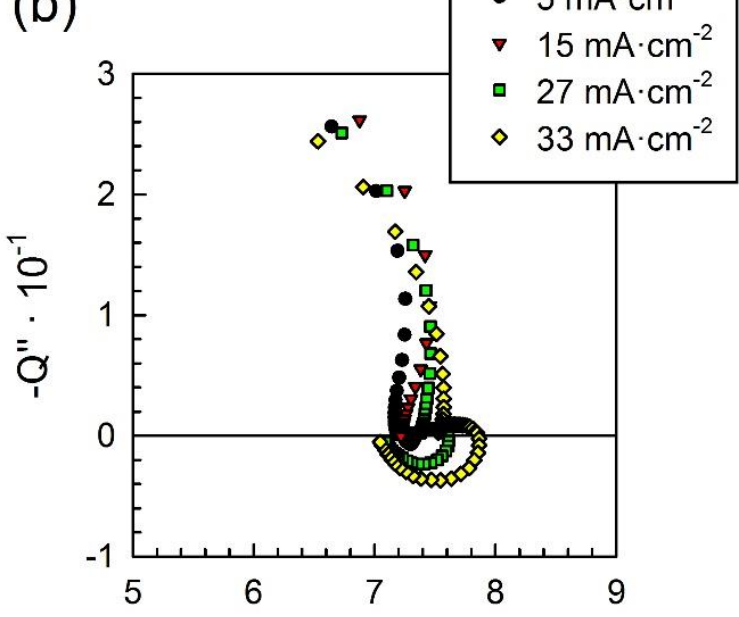

$Q^{\prime} \cdot 10^{-1}$

(d)

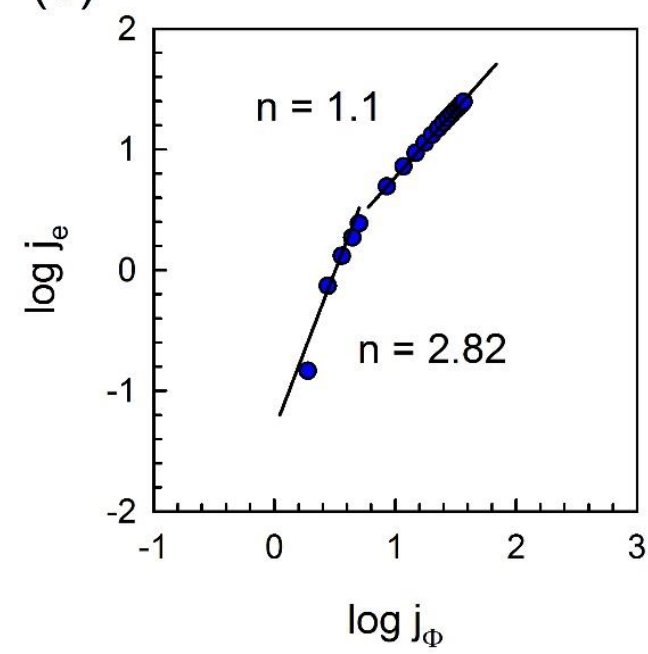

Figure 1 (a) Evolution of extracted photocurrent versus input photon current density from steady state measurements (the inset shows the low intensity region) (b) IMPS spectra for different DC bias blue light intensities expressed as current density as shown in legend. (c) Evolution of EQE as defined from small perturbation methods (EQEPV-DIFF), obtained from steady state measurements (red) and IMPS (orange), and steady state method (green) (EQEPV-ss). (d) $\log -\log$ plot of data in (a) to obtain the exponent $n$ in Equation 3. All measurements carried out with monochromatic radiation of wavelength $470 \mathrm{~nm}$.

Figure 1a shows the evolution of steady state extracted photocurrent versus photon current density, from which the values of $\mathrm{EQE}_{\mathrm{PV}-\mathrm{SS}}$ and $\mathrm{EQE}_{\mathrm{PV} \text {-DIFF }}$ are calculated, shown in Figure 1c. While EQEPv-ss values show a gradual rise to reach a constant value, the

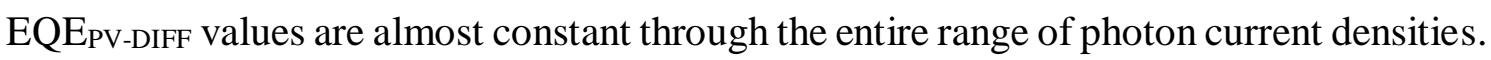
It can be seen that the values of $\mathrm{EQE}_{\mathrm{PV} \text {-DIFF }}$ and $\mathrm{EQE}_{\mathrm{PV}-\mathrm{SS}}$ converge to similar values only 
at high photon current densities. Since EQE measurements are usually carried out at low light intensities (and hence photon current densities), it is clear that the values of EQE will be quite different depending on the employed measuring method. Figure $1 \mathrm{~b}$ shows the corresponding IMPS spectra of the perovskite solar cell obtained at different photon current densities. The spectra typically show two arcs in the upper quadrant, corresponding to high and intermediate frequencies, followed by a low-frequency arc in the lower quadrant, whose origins are discussed later on. For now, we observe that the low frequency limit of the IMPS spectra matches quite nicely with the values of EQEPVDIFF obtained from the slope of the steady state measurements as seen in Figure 1c.

As mentioned earlier, the divergence in values of EQEPV-DIFF and EQESS depend on the exponential factor $n$ in Equation 1. This factor is obtained from a log-log plot of $j_{e}$ versus $j_{\Phi}$, shown in Figure 1d. We can clearly distinguish two regimes of behavior. The first regime occurs at low photon current densities, where the relationship is highly nonlinear with $n=2.8$, while at higher photon current densities, the evolution is almost linear with $n=1.1$. Therefore, the values of $\mathrm{EQE}_{\mathrm{PV} \text {-DIFF }}$ and $\mathrm{EQE}_{\mathrm{SS}}$ collapse only at higher photon current densities. The existence of these regimes can be attributed to the strong photoconductive behavior in perovskites. At high light intensity, a linear behavior is observed as expected, but the low conductivity values near dark conditions ${ }^{18-19}$ reduces charge collection and photocurrent values, so that the strong non-linear dependence at low light intensities (in Fig. 1e) is likely influenced by collection efficiency issues.

We now proceed to clarify the validity of Equation 2 for perovskite solar cells by measuring the effect of the optical chopper frequency on the EQEPV-DIFF values. The EQEPV-DIFF was measured with and without DC white light bias at five different optical chopper frequencies of 500, 400, 286, 110 and $14 \mathrm{~Hz}$. Figure $2 \mathrm{a}$ and $2 \mathrm{~b}$ shows the variation in EQEPV-DIFF without and with a $10 \mathrm{~mW} \cdot \mathrm{cm}^{-2} \mathrm{DC}$ white light bias at a given frequency of the optical chopper with respect to the values at $500 \mathrm{~Hz}$, which is used as reference. We observe that the EQEPV-DIFF increases upon lowering the optical chopper frequency in both cases, with a maximum increment of 8-10\% in EQEPV-DIFF from $500 \mathrm{~Hz}$ to $110 \mathrm{~Hz}$. At lower frequencies $(14 \mathrm{~Hz})$ the EQEPV-DIFF drops. This strong frequency dependence describing a response peak is also reflected in the IMPS spectra of Figure $2 \mathrm{c}$ that shows high frequency and intermediate arcs in the first quadrant followed by a low frequency arc in the fourth quadrant. The evolution of the real part of the IMPS transfer function is shown in Figure $2 \mathrm{~d}$. It shows a maxima at high frequency $\left(\sim 10^{4} \mathrm{~Hz}\right)$ and another at low frequency $(\sim 1 \mathrm{~Hz})$, with very little evolution in between. After the second maxima, it then drops sharply for very low frequencies. It is then evident that both techniques (EQEPV-DIFF and IMPS) exhibit similar evolution with the perturbation frequency. This is because EQEPV-DIFF and $Q$ ', the real part of IMPS, provides information on the effective extracted photocurrent at a given perturbation frequency. 

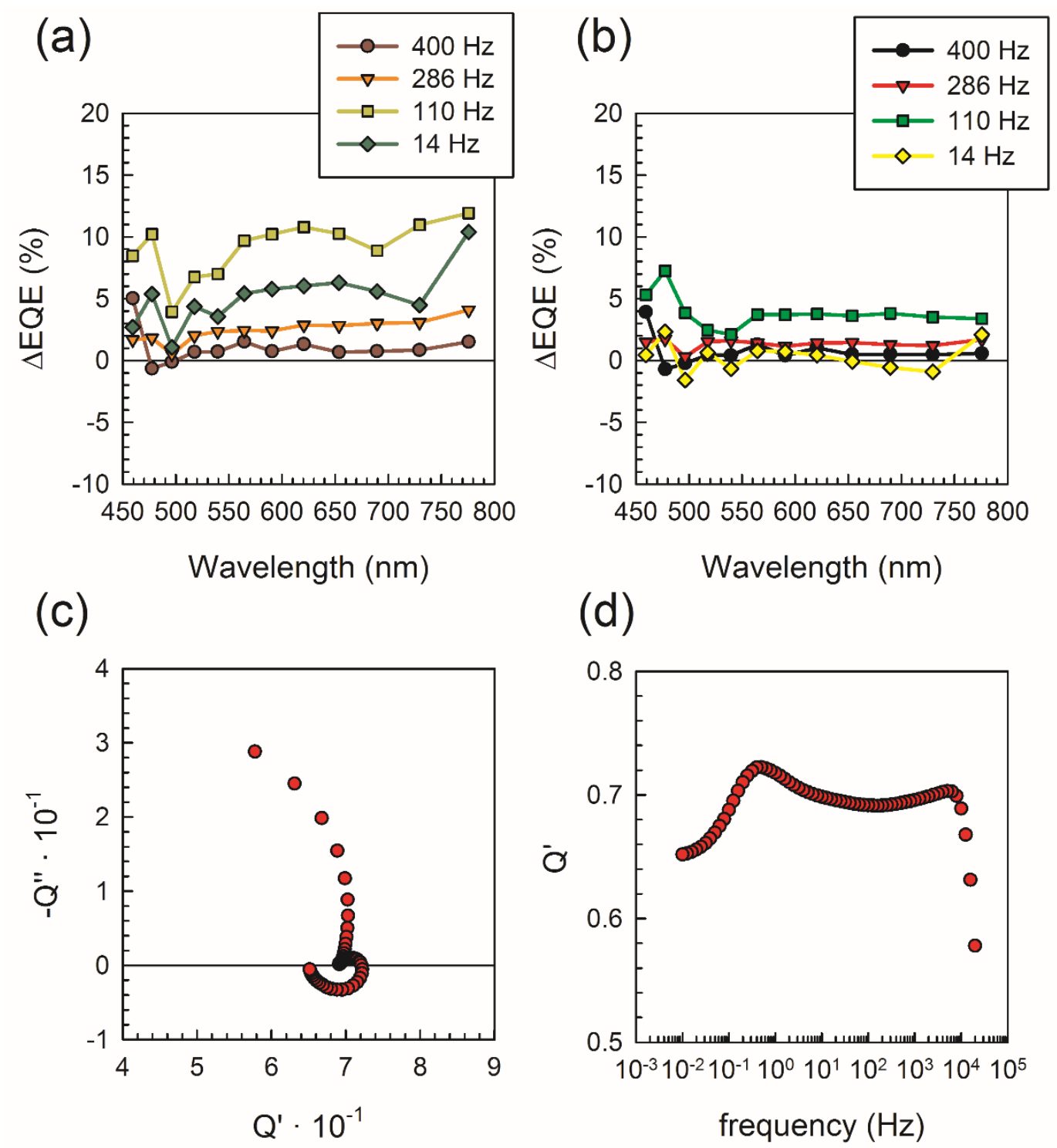

Figure 2 Variation in EQEPV-DIFF at (a) $0 \mathrm{DC}$ white light bias and (b) $10 \mathrm{~mW} \cdot \mathrm{cm}^{-2} \mathrm{DC}$ white light bias for different optical chopper frequencies with respect to spectra measured at $500 \mathrm{~Hz}$. (c) IMPS spectra measured at $33 \mathrm{~mA} \cdot \mathrm{cm}^{-2} \mathrm{DC}$ blue light bias and (d) Evolution of real part of IMPS transfer function versus frequency at $470 \mathrm{~nm}$.

In general, the value of EQEPV-DIFF obtained from direct measurements is in fact the real part of the IMPS transfer function at the given optical chopper frequency, as mentioned earlier. For the simplest model of a solar cell, consisting of a recombination resistance $\left(R_{r e c}\right)$ and a chemical capacitance $\left(C_{\mu}\right)$ coupled in parallel with a current source, and a series resistance $\left(R_{s}\right)$, the IMPS response is an arc in the upper quadrant and the real part of the IMPS transfer function is a frequency-dependent process, containing the ratio between the frequency $\omega_{\tau}$ associated to the time constant $\tau=R_{s} C_{\mu}$ and the applied frequency (see SI for derivation). In this case, 


$$
Q^{\prime}(\omega)=\frac{Q(0)}{1+Q(0)^{2}\left(\omega / \omega_{\tau}\right)^{2}}
$$

where the low frequency intercept has the value

$$
Q(0)=\left(1+\frac{R_{s}}{R_{r e c}}\right)^{-1}
$$

This can be understood by observing the equivalent circuit of the simple solar cell model at SC shown in Figure 3a, with a current source corresponding to photogenerated carriers in parallel to both the recombination resistor and the chemical capacitor. The IMPS response can be viewed similar to an $\mathrm{RC}$ attenuator, where the capacitor controls the frequency-dependent response of the extracted photocurrent through the series resistance. ${ }^{20}$ Therefore, the IMPS arc is the attenuation of the total photocurrent through the equipotential lines in the circuit. At higher frequencies, the capacitor acts as a short circuit and reduces the flow of current through the series resistance, while at low frequencies, the capacitor behaves as a blocking element and the total current through the series resistance is determined simply by the ratio of magnitudes of the series resistance and the recombination resistance. Therefore, the real part of the IMPS transfer function and hence the EQEPV-DIFF has a maximum at zero frequency corresponding to Equation 8 and reduces with increasing frequency for the simple solar cell model. This leads to the important fact that the EQE associated with the time constant $\tau=R_{s} C_{\mu}$ is much larger than the optical chopper frequency employed.

(a)
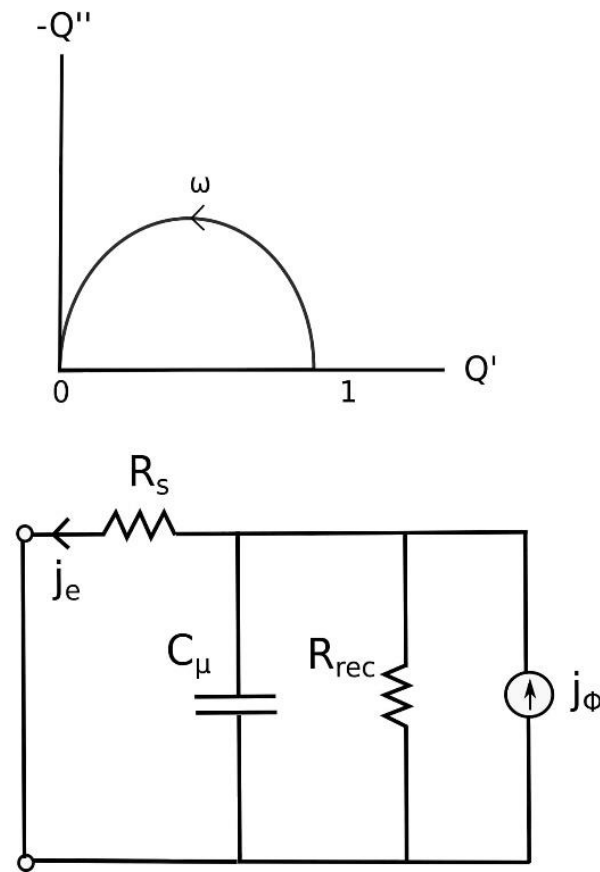

(b)
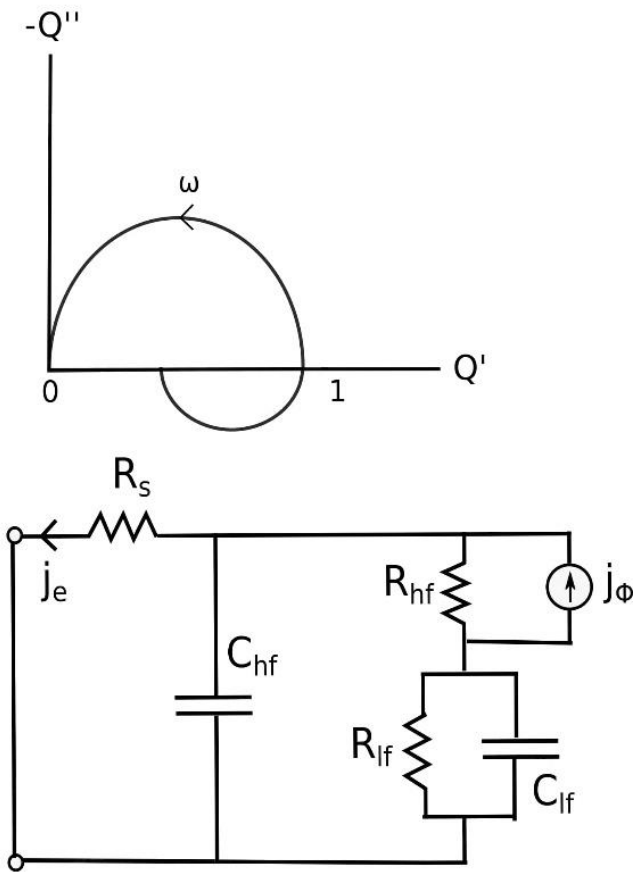
Figure 3 IMPS response at SC and corresponding equivalent circuit for (a) basic solar cell model and (b) perovskite solar cell.

In perovskite solar cells, the IMPS spectra in Figure $1 \mathrm{~b}$ deviate from the previous simple model, with the observation of a distinctive low frequency arc in the lower quadrant. The high frequency arc can be attributed, as explained earlier, to the time constant formed by the series resistance and a high frequency capacitor, ${ }^{21}$ as shown in the equivalent circuit of Figure 3b, which corresponds to that commonly used to analyze impedance spectroscopy responses. ${ }^{15}$ The high-frequency capacitance is well established to be the bulk dielectric capacitance of the perovskite. ${ }^{16}$ The IMPS low-frequency arc is related to the components in the equivalent circuit as far as the generation of carriers is located exclusively across the high frequency resistor $R_{h f}$, as calculated using standard electrical network analysis. This creates a delay in the extracted photocurrent through the low-frequency capacitance $C_{\text {lf }}$ with respect to the photogenerated current, leading to a response in the lower quadrant. At the low-frequency limit, the separated localization of the current source forces the resistor $R_{l f}$ to act identically as an extra series resistor, which can be seen simply as the basic solar cell model with a net series resistance of $R_{n e t}=R_{s}+R_{l f}$. This reduces the value of EQEPV-DifF as can be seen from Equation 8, which causes the arc in the fourth quadrant to evolve towards lower EQEPV-DIFF values at lower frequencies. Therefore, the evolution of the magnitude of the low-frequency resistor $R_{l f}$ with light intensity is crucial to determine the achievable low frequency EQEPV-DIFF values. It must be noted that the large low frequency capacitance associated with this resistor in the order of $\mathrm{mF} \mathrm{cm}^{-2}$ at $\mathrm{SC}^{15}$ has recently been ascribed to the accumulation of minority electrons and anions at the perovskite/HTL interface. ${ }^{22}$ Therefore, a further understanding of the properties of the low frequency resistor and capacitor are fundamental to understanding the nature of the EQEPV-DIFF in perovskite solar cells.

In summary, some years back, the drift of efficiencies of perovskite solar cells by hysteresis effects could be identified. An ensuring point to a reliable measurement of performance is to calculate the expected photocurrent from independent EQE measurement. But here, we show that the latter effect can produce a disparity of results. To clarify the question, we provided formal definitions of the EQE for solar cells as can be obtained from steady state measurements or from small perturbation methods. The obtained values can vary strongly from each other depending on the linearity of the relationship between the extracted photocurrent and the input photon flux. The extremely slow response in timescale of seconds of perovskite solar cells at short circuit is inevitably reflected in small perturbation EQE measurements, where variations as high as $10 \%$ can be obtained with optical chopper frequencies in the range of 10-500 Hz. Through IMPS measurements, we shed light on the frequency-dependent evolution of the external quantum efficiency, which reaches a maximum at intermediate frequencies followed by a reduction at low frequencies. This low frequency effect is ascribed to the differences in charge generation within the perovskite solar cell. Due to the singular evolution of the 
EQE, it is imperative to establish protocols and to provide detailed information regarding the method of measurement. Measurements with several optical chopper frequencies, stated explicitly, are recommended to avoid erroneous estimation of the true EQE.

\section{Acknowledgements}

We acknowledge funding from MINECO of Spain under project MAT2016-76892C3-1-R and MAT2016-76892-C3-2-R. P.P.B. thanks MINECO for his post-doctoral RyC contract and acknowledges financial support from the Conselleria d'Educació Investigació, Cultura i Esport, Generalitat Valenciana (SEJI2017/2017/012).

\section{References}

1. Bisquert, J. The Physics of Solar Cells: Perovskites, Organics, and Photovoltaic Fundamentals. CRC Press: 2017.

2. Soedergren, S.; Hagfeldt, A.; Olsson, J.; Lindquist, S.-E. Theoretical Models for the Action Spectrum and the Current-Voltage Characteristics of Microporous Semiconductor Films in Photoelectrochemical Cells. The Journal of Physical Chemistry 1994, 98 (21), 5552-5556.

3. Thomas, J. K. B.; Yana, V.; Zhe, L.; Dinesh, K.; Richard, H. F.; Christopher, R. M. White-light bias external quantum efficiency measurements of standard and inverted P3HT : PCBM photovoltaic cells. Journal of Physics D: Applied Physics 2012, 45 (41), 415101.

4. Li, X.; Bi, D.; Yi, C.; Décoppet, J.-D.; Luo, J.; Zakeeruddin, S. M.; Hagfeldt, A.; Grätzel, M. A vacuum flash-assisted solution process for high-efficiency large-area perovskite solar cells. Science 2016, 353 (6294), 58-62.

5. Tan, H.; Jain, A.; Voznyy, O.; Lan, X.; García de Arquer, F. P.; Fan, J. Z.; Quintero-Bermudez, R.; Yuan, M.; Zhang, B.; Zhao, Y.; Fan, F.; Li, P.; Quan, L. N.; Zhao, Y.; Lu, Z.-H.; Yang, Z.; Hoogland, S.; Sargent, E. H. Efficient and stable solutionprocessed planar perovskite solar cells via contact passivation. Science 2017, 355 (6326), 722.

6. Cowan, S. R.; Wang, J.; Yi, J.; Lee, Y.-J.; Olson, D. C.; Hsu, J. W. Intensity and wavelength dependence of bimolecular recombination in P3HT: PCBM solar cells: A white-light biased external quantum efficiency study. Journal of Applied Physics 2013, $113(15), 154504$

7. Tsai, H.; Asadpour, R.; Blancon, J.-C.; Stoumpos, C. C.; Durand, O.; Strzalka, J. W.; Chen, B.; Verduzco, R.; Ajayan, P. M.; Tretiak, S.; Even, J.; Alam, M. A.; Kanatzidis, M. G.; Nie, W.; Mohite, A. D. Light-induced lattice expansion leads to high-efficiency perovskite solar cells. Science 2018, 360 (6384), 67.

8. Bush, K. A.; Palmstrom, A. F.; Yu, Z. J.; Boccard, M.; Cheacharoen, R.; Mailoa, 
J. P.; McMeekin, D. P.; Hoye, R. L. Z.; Bailie, C. D.; Leijtens, T.; Peters, I. M.; Minichetti, M. C.; Rolston, N.; Prasanna, R.; Sofia, S.; Harwood, D.; Ma, W.; Moghadam, F.; Snaith, H. J.; Buonassisi, T.; Holman, Z. C.; Bent, S. F.; McGehee, M. D. 23.6\%-efficient monolithic perovskite/silicon tandem solar cells with improved stability. Nature Energy 2017, 2, 17009.

9. Nie, W.; Tsai, H.; Blancon, J. C.; Liu, F.; Stoumpos Costas, C.; Traore, B.; Kepenekian, M.; Durand, O.; Katan, C.; Tretiak, S.; Crochet, J.; Ajayan Pulickel, M.; Kanatzidis Mercouri, G.; Even, J.; Mohite Aditya, D. Critical Role of Interface and Crystallinity on the Performance and Photostability of Perovskite Solar Cell on Nickel Oxide. Advanced Materials 2017, 30 (5), 1703879.

10. Gottesman, R.; Lopez-Varo, P.; Gouda, L.; Jimenez-Tejada, J. A.; Hu, J.; Tirosh, S.; Zaban, A.; Bisquert, J. Dynamic Phenomena at Perovskite/Electron-Selective Contact Interface as Interpreted from Photovoltage Decays. Chem 2016, 1 (5), 776-789.

11. Unger, E.; Hoke, E.; Bailie, C.; Nguyen, W.; Bowring, A.; Heumüller, T.; Christoforo, M.; McGehee, M. Hysteresis and transient behavior in current-voltage measurements of hybrid-perovskite absorber solar cells. Energy \& Environmental Science 2014, 7 (11), 3690-3698.

12. O’Regan, B. C.; Barnes, P. R. F.; Li, X.; Law, C.; Palomares, E.; Marin-Beloqui, J. M. Optoelectronic Studies of Methylammonium Lead Iodide Perovskite Solar Cells with Mesoporous TiO2: Separation of Electronic and Chemical Charge Storage, Understanding Two Recombination Lifetimes, and the Evolution of Band Offsets during J-V Hysteresis. Journal of the American Chemical Society 2015, 137 (15), 5087-5099.

13. Samu, G. F.; Janáky, C.; Kamat, P. V. A Victim of Halide Ion Segregation. How Light Soaking Affects Solar Cell Performance of Mixed Halide Lead Perovskites. ACS Energy Letters 2017, 2 (8), 1860-1861.

14. Lopez-Varo, P.; Jiménez-Tejada, J. A.; García-Rosell, M.; Ravishankar, S.; Garcia-Belmonte, G.; Bisquert, J.; Almora, O. Device Physics of Hybrid Perovskite Solar cells: Theory and Experiment. Advanced Energy Materials 2018.

15. Zarazua, I.; Han, G.; Boix, P. P.; Mhaisalkar, S.; Fabregat-Santiago, F.; MoraSeró, I.; Bisquert, J.; Garcia-Belmonte, G. Surface Recombination and Collection Efficiency in Perovskite Solar Cells from Impedance Analysis. The Journal of Physical Chemistry Letters 2016, 7 (24), 5105-5113.

16. Almora, O.; Zarazua, I.; Mas-Marza, E.; Mora-Sero, I.; Bisquert, J.; GarciaBelmonte, G. Capacitive Dark Currents, Hysteresis, and Electrode Polarization in Lead Halide Perovskite Solar Cells. The Journal of Physical Chemistry Letters 2015, 6 (9), 1645-1652.

17. Bertoluzzi, L.; Bisquert, J. Investigating the consistency of models for water 
splitting systems by light and voltage modulated techniques. The Journal of Physical Chemistry Letters 2016, 8 (1), 172-180.

18. Chen, Y.; Yi, H.; Wu, X.; Haroldson, R.; Gartstein, Y.; Rodionov, Y.; Tikhonov, K.; Zakhidov, A.; Zhu, X.-Y.; Podzorov, V. Extended carrier lifetimes and diffusion in hybrid perovskites revealed by Hall effect and photoconductivity measurements. Nature Communications 2016, 7, 12253.

19. Wang, F.; Mei, J.; Wang, Y.; Zhang, L.; Zhao, H.; Zhao, D. Fast Photoconductive Responses in Organometal Halide Perovskite Photodetectors. ACS Applied Materials \& Interfaces 2016, 8 (4), 2840-2846.

20. Ponomarev, E.; Peter, L. A generalized theory of intensity modulated photocurrent spectroscopy (IMPS). Journal of Electroanalytical Chemistry 1995, 396 (1-2), 219-226.

21. Pockett, A.; Eperon, G. E.; Peltola, T.; Snaith, H. J.; Walker, A.; Peter, L. M.; Cameron, P. J. Characterization of planar lead halide perovskite solar cells by impedance spectroscopy, open-circuit photovoltage decay, and intensity-modulated photovoltage/photocurrent spectroscopy. The Journal of Physical Chemistry C 2015, 119 (7), 3456-3465.

22. Garcia-Rosell, M.; Bou, A.; Jiménez-Tejada, J. A.; Bisquert, J.; Lopez-Varo, P. Analysis of the Influence of Selective Contact Heterojunctions on the Performance of Perovskite Solar Cells. The Journal of Physical Chemistry C 2018. 


\title{
Effects of Frequency Dependence of the External Quantum Efficiency of Perovskite Solar Cells
}

\author{
Sandheep Ravishankar, ${ }^{1 *}$ Clara Aranda, ${ }^{1}$ Pablo.P.Boix, ${ }^{2}$ Juan A.Anta, ${ }^{3}$ Juan \\ Bisquert $^{1 *}$ and Germà Garcia-Belmonte ${ }^{1 *}$
}

\author{
${ }^{1}$ Institute of Advanced Materials (INAM), Universitat Jaume I, 12006 Castelló, Spain \\ ${ }^{2}$ Instituto de Ciencia Molecular (ICMol), Universidad de Valencia, Catedrático José \\ Beltran, 2, 46980 Paterna, Spain \\ ${ }^{3}$ Department of Physical, Chemical and Natural systems, University Pablo de Olavide, \\ 41013, Sevilla, Spain
}

Email: ravishan@uji.es, bisquert@uji.es,garciag@uji.es

\section{Supporting Information}

\section{Materials and precursor solutions}

All materials were used as received: FTO glasses $(25 \times 25 \mathrm{~mm}$, Pilkington TEC15, $\sim 15 \Omega /$ sq resistance), $\mathrm{TiO}_{2}$ paste (Dyesol 30NRD, $300 \mathrm{~nm}$ average particle size), $\mathrm{CH}_{3} \mathrm{NH}_{3} \mathrm{I}$ (DYESOL), $\mathrm{PbI}_{2}$ (TCI, $99.99 \%$ ), titanium diisopropoxidebis (acetylacetonate) (75\% in isopropanol, Sigma-Aldrich), spiro-OMeTAD (Merck). The perovskite precursor solution was prepared by reacting DMF solutions (50 wt \%) containing MAI and $\mathrm{PbI}_{2}$ $(1: 1 \mathrm{~mol} \%)$ and MAI, PbI2 and DMSO (1:1:1 mol \%) and for the different humidities, the molar ratio of DMSO was changed from $0 ; 0.5 ; 0.75 ; 1 ; 1.25 ; 1.5$. The spiro-OMeTAD solution was prepared by dissolving in $1 \mathrm{~mL}$ of chlorobenzene $72.3 \mathrm{mg}$ of $\left(2,2^{\prime}, 7,7^{\prime}\right.$ tetrakis(N,N'-di-p-methoxyphenylamine)-9,9'-spirobifluorene), $28.8 \mu \mathrm{L}$ of 4-tertbutylpyridine, and $17.5 \mu \mathrm{L}$ of a stock solution of $520 \mathrm{mg} / \mathrm{mL}$ of lithium bis(trifluoromethylsulfonyl) imide in acetonitrile.

\section{Photovoltaic device fabrication}

All the processes were carried out outside the glovebox in ambient conditions at different humidity levels. Photovoltaic devices were prepared over FTO glasses, which were partially etched with zinc powder and $\mathrm{HCl}(2 \mathrm{M})$. The substrates were cleaned with soap (Hellmanex) and rinsed with Milli-Q water and ethanol. Then, the substrates were sonicated for $15 \mathrm{~min}$ in a solution of acetone:isopropanol $(1: 1 \mathrm{v} / \mathrm{v})$, rinsed with ethanol and Milli-Q water, and dried with compressed air. After that, the substrates were treated in a UV-O3 chamber for $15 \mathrm{~min}$. The TiO2 blocking layer was deposited onto the substrates by spray pyrolysis at $450{ }^{\circ} \mathrm{C}$, using a titanium diisopropoxide bis(acetylacetonate) solution diluted in ethanol (1:9, v/v), with oxygen as carrier gas. The spray was performed in 3 steps of $6 \mathrm{~s}$, spraying each time $5 \mathrm{~mL}$ (approx.), and waiting 30 $\mathrm{s}$ between steps. After the spraying process, the films were kept at $450{ }^{\circ} \mathrm{C}$ for $30 \mathrm{~min}$. The 
mesoporous $\mathrm{TiO}_{2}$ layer was deposited by spin coating at $2000 \mathrm{rpm}$ during $10 \mathrm{~s}$ using a $\mathrm{TiO}_{2}$ paste diluted in ethanol (1:5, weight ratio). After drying at $100{ }^{\circ} \mathrm{C}$ for $10 \mathrm{~min}$, the $\mathrm{TiO}_{2}$ mesoporous layer was heated at $500{ }^{\circ} \mathrm{C}$ for $30 \mathrm{~min}$ and later cooled to room temperature. Before the deposition of perovskite films, $100 \mu \mathrm{l}$ of a lithium bis(trifluoromethylsulfonyl) imide $35 \mathrm{mM}$ solution in acetonitrile is prepared and deposited on the top of the device by spin-coating at $3000 \mathrm{rpm}(2000 \mathrm{ac})$ for 10 seconds. After that, the substrates are heated up at $450^{\circ} \mathrm{C}$ for $30 \mathrm{~min}$. The perovskite precursor solution $(50$ $\mu \mathrm{L}$ ) was spin-coated at $4000 \mathrm{rpm}$ for $50 \mathrm{~s}$. DMF is selectively washed with non-polar diethyl ether while one-step spin- coating at $4000 \mathrm{rpm}$ is carried out. The diethyl ether needs to be added just before the white solid begins to crystallize in the substrate. At 30 $\%$ R.H. the diethyl ether is added after 8-9 seconds of the substrate spinning and at $60 \%$ R.H. after 12-13 seconds. After deposition, the substrate was annealed at $100{ }^{\circ} \mathrm{C}$ for 3 min. Then, the perovskite films were covered with the hole-transporting material (HTM) by dynamic spin coating at $4000 \mathrm{rpm}$ for $30 \mathrm{~s}$, using $50 \mu \mathrm{L}$ of spiro- OMeTAD solution. Finally, $60 \mathrm{~nm}$ of gold was thermally evaporated on top of the device to form the electrode contacts using a commercial Univex 250 chamber, from Oerlikon Leybold Vacuum.

\section{EQE measurements}

Measurements of EQE were made using a Newport APEX2-QTH lamp connected to an Oriel 1/8 m Cornerstone Monochromator. The light intensity was modulated by a New Focus 3502 Optical Chopper, which also triggered the measurement of the cell response with a lock-in amplifier (SR830 DSP by Stanford Research Systems).

\section{IMPS measurements}

IMPS measurements were carried out using a blue (470 nm) LED supplied by Philips LUMILEDS, and the distance between the sample and LED was adjusted to obtain the range of DC bias light intensities necessary, calibrated using a Si photodiode. The LED was driven using an Autolab LED driver through an Autolab potentiostat. In all cases, the perturbation was $10 \%$ of the DC bias light intensity. The range of frequencies investigated was $20 \mathrm{kHz}-10 \mathrm{mHz}$. 
(a)

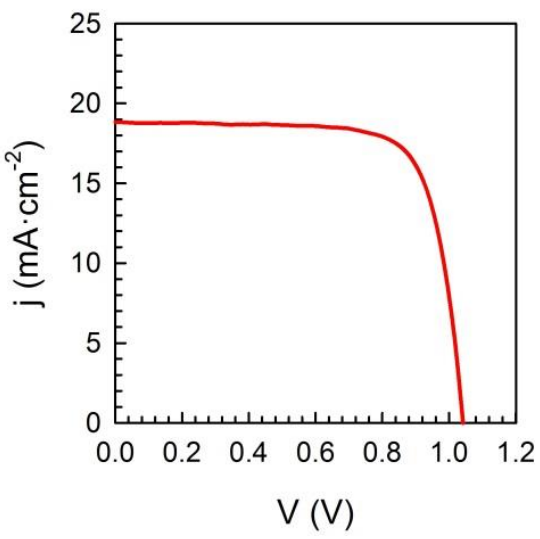

(b)

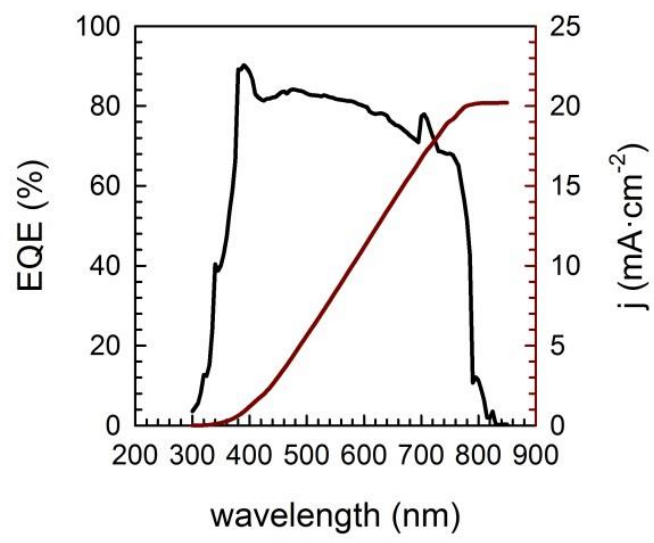

Figure SI1 (a) $j-V$ curve of the perovskite solar cell used for EQE and IMPS measurements, with an efficiency of $14.79 \%$. (b) Measured EQE spectra at zero DC bias yielding a maximum short-circuit current density of $20.2 \mathrm{~mA} \cdot \mathrm{cm}^{-2}$.

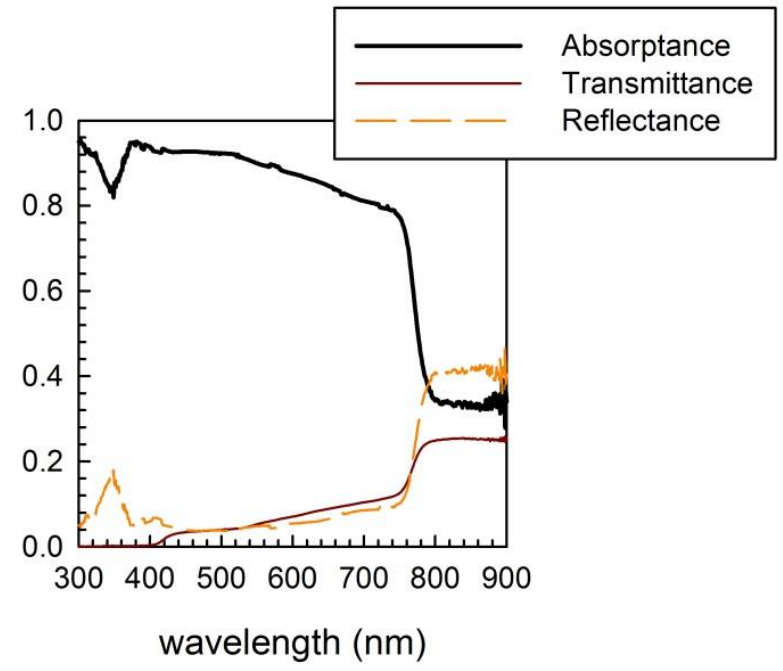

Figure SI2 Absorptance of the perovskite solar cell used for EQE and IMPS measurements obtained from transmittance and reflectance measurements.

\section{IMPS basic solar cell model}

We assume a solar cell of length $L$ with electrons as minority carriers, with electron and hole selective contacts at $x=0$ and $x=L$ respectively. The transport equation is then given by 


$$
\frac{d n}{d t}=\frac{1}{q} \frac{d j_{e}}{d x}+G-U
$$

where $j_{e}$ is the current density of electrons and $G$ and $U$ are the generation and recombination terms, respectively. We assume the transport of electrons is excellent, therefore a very small gradient in concentration of electrons is required to drive the current. Therefore, $n$ is position independent, and we can integrate Equation S1 over the length of the semiconductor $L$ to yield

$$
\frac{d n}{d t}=\frac{j_{e}(L)-j_{e}(0)}{q L}+G-U
$$

We consider the current for the electrons to be blocked at $x=L$, and we have

$$
j_{e}(L)=0
$$

Combining Equations S2 and S3, we have

$$
\frac{d n}{d t}=-\frac{j_{e}}{q L}+\frac{j_{\Phi}}{q L}-U
$$

At steady state, assuming total absorption, we have

$$
\overline{j_{e}}=\overline{j_{\Phi}}-q L \bar{U}
$$

where steady state values are indicated by an overbar. We now apply a small perturbation to the variables in Equation S4 from a given steady state as

$$
\frac{d(\bar{n}+\widetilde{n})}{d t}=-\frac{\overline{j_{e}}+\tilde{j}_{e}}{q L}+\frac{\overline{j_{\Phi}}+\tilde{j}_{\Phi}}{q L}-(\bar{U}+\widetilde{U})
$$

Combining Equations S6 and S5

$$
\frac{d \tilde{n}}{d t}=-\frac{\tilde{J}_{e}}{q L}+\frac{\tilde{J}_{\Phi}}{q L}-\tilde{U}
$$

We have the Boltzmann expression for the electron density and linear recombination of minority carriers as

$$
\begin{aligned}
& \bar{n}=n_{0} e^{\frac{q\left(\bar{V}+R_{s} \overline{j_{e}}\right)}{k_{B} T}} \\
& \bar{U}=B_{r e c}\left(\bar{n}-n_{0}\right)
\end{aligned}
$$

The IMPS transfer function involves the measurement of modulated extracted photocurrent versus modulated input photon current density

$$
Q(\omega)=\frac{\hat{j}_{e}}{\hat{j}_{\Phi}}
$$

where the hat represents a function in the frequency domain. Therefore, we can express 
the perturbed quantities of Equations S8 and S9 as

$$
\begin{aligned}
& \tilde{n}=\left(\frac{d \bar{n}}{d \overline{j_{e}}}\right) \tilde{j}_{e} \\
& \tilde{U}=\left(\frac{d \bar{U}}{d \bar{n}}\right)\left(\frac{d \bar{n}}{d \overline{j_{e}}}\right) \tilde{j}_{e}
\end{aligned}
$$

From differentiating Equations S8, S9 and substituting in Equations S10, S11, we have

$$
\begin{aligned}
& \tilde{n}=\left(\frac{q R_{s} \bar{n}}{k_{B} T}\right) \tilde{j}_{e} \\
& \tilde{U}=\left(\frac{B_{r e c} q R_{s} \bar{n}}{k_{B} T}\right) \tilde{J}_{e}
\end{aligned}
$$

Substituting Equations S13, S14 in Equation S7, taking the Laplace transform and rearranging terms, we have

$$
\left(i \omega \frac{q^{2} L R_{s} \bar{n}}{k_{B} T}+\frac{B_{r e c} q^{2} L R_{s} \bar{n}}{k_{B} T}+1\right) \hat{j}_{e}=\hat{j}_{\Phi}
$$

We define the chemical capacitance and recombination resistance from differentiating equations S8 and S9 with respect to voltage as

$$
\begin{aligned}
& C_{\mu}=\frac{q^{2} L \bar{n}}{k_{B} T} \\
& R_{r e c}=\frac{k_{B} T}{q^{2} \operatorname{Ln} B_{r e c}}
\end{aligned}
$$

Substituting Equations S16 and S17 in S15 and rearranging terms, we have

$$
Q(\omega)=\left(1+\frac{R_{s}}{R_{r e c}}+i \omega R_{s} C_{\mu}\right)^{-1}
$$

The real part of the IMPS transfer function is then

$$
Q^{\prime}(\omega)=\frac{Q(0)}{1+Q(0)^{2}\left(\omega / \omega_{\tau}\right)^{2}}
$$

where $\omega_{\tau}=\left(R_{s} C_{\mu}\right)^{-1}$ and the low frequency intercept is given by

$$
Q(0)=\left(1+\frac{R_{s}}{R_{r e c}}\right)^{-1}
$$




\section{Chapter 8}

\section{Publication 5}

Perovskite Solar Cell Modeling using Light and Voltage Modulated Techniques

Ravishankar, S.; Aranda, C.; Sanchez, S.; Bisquert, J.; Saliba, M.; Garcia-Belmonte, G. Perovskite Solar Cell Modeling Using Light and Voltage Modulated Techniques. The Journal of Physical Chemistry C 2019. Reprinted with permission, Copyright 2019, American Chemical Society.

\section{Candidate's contribution}

\begin{tabular}{|l|l|}
\hline Nature of Contribution & Extent of Contribution \\
\hline - Carried out all the measurements & \\
- Developed the model and carried & \\
- $\begin{array}{l}\text { out the simulations } \\
\text { - Wrote first draft of the manuscript }\end{array}$ & $80 \%$ \\
- Wrote first draft of the reply to & \\
referees &
\end{tabular}

\section{Introduction}

This chapter provides a connection between the model developed in Chapter 4,5 and 6 based on capacitance information from IS measurements and the internal displacement of photovoltage at SC conditions identified from IMPS measurements in the previous chapter. This connection is the underlying EC of the PSC, obtained from IS and IMPS measurements at $\mathrm{OC}$ conditions.

\section{Published manuscript}




\title{
Perovskite Solar Cell Modeling using Light and Voltage Modulated Techniques
}

\section{Sandheep Ravishankar, ${ }^{1}$ Clara Aranda, ${ }^{1}$ Sandy Sanchez, ${ }^{2}$ Juan Bisquert, ${ }^{1}$ Michael Saliba, ${ }^{* 2}$ and Germà Garcia-Belmonte ${ }^{1 *}$}

${ }^{1}$ Institute of Advanced Materials (INAM), Universitat Jaume I, 12006 Castelló, Spain

${ }^{2}$ Adolphe Merkle Institute, Chemin des Verdiers 4, CH-1700, Fribourg, Switzerland

Email: michael.saliba@unifr.ch, garciag@uji.es

\begin{abstract}
The analysis of the Impedance Spectroscopy data of perovskite solar cells (PSCs) has been challenging so far, with the low frequency phenomena in particular yielding ambivalent results and interpretation. We tackle this problem by carrying out Intensity Modulated Photocurrent Spectroscopy (IMPS) measurements at open circuit (OC) on $\mathrm{CH}_{3} \mathrm{NH}_{3} \mathrm{PbBr}_{3}$ cells prepared by the flash infra-red annealing method with different electron selective contacts. We identify the existence of a capacitance of the order of $10^{-}$ ${ }^{4} \mathrm{~F} \cdot \mathrm{cm}^{-2}$ that is not discernible from Impedance Spectroscopy (IS) measurements, which we attribute to an accumulation of anions at the perovskite/Spiro-OMeTAD interface, which also likely includes an electronic component. This interface is a dominant recombination pathway at lower voltages and can account for the large disparity in fill factors observed in PSCs. By developing detailed models for the IMPS response at both $\mathrm{OC}$ and short circuit (SC) conditions, we also confirm that the arcs observed in the upper quadrant of the IMPS spectra are not related to transport times, as is commonly interpreted, but time constants formed between the series resistance and capacitors within the circuit. By combining insights from IMPS and IS measurements, we develop a more complete equivalent circuit for the PSC that can be used as a basis for coming research with different perovskite materials and contact layers.
\end{abstract}




\section{Introduction}

Perovskite solar cells (PSCs) have shown rapid improvements in efficiencies (above $20 \%$ ) over the last few years with the potential for a prominent role in clean energy conversion in the future. However, several working aspects of PSCs are unclear, such as the dominant recombination mechanisms at different voltages, which are coupled to the ionic movement within the perovskite ${ }^{1-2}$ and the role of interfaces in carrier extraction and their relation to charge accumulation. This hinders identifying the limiting mechanisms that need to be overcome to further advance efficiencies and stability. A prominent technique that allows discerning internal processes that occur on different timescales is Impedance Spectroscopy (IS) that measures the current response upon the application of an $\mathrm{AC}$ voltage perturbation, scanned over a range of frequencies.

However, the IS response of PSCs has been quite challenging to interpret with a variety of observed spectra. While the most common spectra has been the observation of two arcs at open circuit (OC) conditions, ${ }^{3-5}$ there have also been exotic observations of three arcs, inductive loops ${ }^{6}$ and also negative capacitance, ${ }^{7}$ where the imaginary part of the impedance $Z$ enters the fourth quadrant of a complex plane plot $\left(Z^{\prime},-Z^{\prime \prime}\right)$. While the high frequency (HF) capacitance is now well understood to be the bulk dielectric capacitance, ${ }^{8}$ the connection between the large low frequency (LF) capacitance and the HF and LF resistances is yet to be discerned, compounded by the fact that the LF data is often noisy. ${ }^{9}$

In order to obtain further knowledge about the recombination and transport mechanisms and complement the insights obtained from IS, we carry out Intensity Modulated Photocurrent Spectroscopy (IMPS) measurements at OC conditions on stable and reproducible $\mathrm{CH}_{3} \mathrm{NH}_{3} \mathrm{PbBr}_{3}\left(\mathrm{MAPbBr}_{3}\right)$ cells prepared by flash infra-red annealing ${ }^{10-}$ 11 (FIRA) with different electron selective contact (ETL) layers. IMPS involves the measurement of the current response of the solar cell to a modulated input of light excitation. Upon the application of a modulated current density $\hat{j}_{\Phi}$ related to photogeneration by incident photon flux, the modulated extracted electronic current $\hat{j}_{e}$ is monitored and the transfer function is defined as ${ }^{12}$

$$
Q(\omega)=\frac{\hat{j}_{e}}{\hat{j}_{\Phi}}
$$

The real and imaginary parts are denoted $Q^{\prime}$ and $Q^{\prime \prime}$ respectively. IMPS was used extensively for photoelectrochemistry ${ }^{13}$ and dye-sensitized solar cells. In the case of PSCs, the IMPS spectra have generally been seen to show two or three arcs in the upper quadrant of a complex $Q$-plane plot. While the HF arc is attributed to the time constant formed between the series resistance and the geometric capacitance, ${ }^{14-15}$ the secondary arcs in the upper quadrant have predominantly been ascribed to transport processes, ${ }^{16}$ such as transport of electrons in the $\mathrm{TiO}_{2}{ }^{17-18}$ or ionic movement. ${ }^{19-20}$ Historically, diffusion-limited processes, where the transport frequency is lower than the perturbation frequency, a 45 degree line from the origin is observed, both in IMPS and IS. ${ }^{21-22}$ Diffusion has been shown recently to be the dominant electronic transport mechanism in 
high efficiency $\mathrm{PSCs}^{23}$ and hence, the absence of its characteristic signal in small perturbation methods for PSCs raises serious doubts over the above mentioned interpretations. Furthermore, there have been observations of a LF arc in the lower quadrant, attributed generally to interfacial transport and recombination, ${ }^{14}$ which, as clarified in our previous paper, was related to the separation of the internal photovoltage upon generation of carriers in the perovskite. ${ }^{15}$

It was shown previously that the IS and IMPS responses should give exactly the same information. ${ }^{12}$ Indeed both transfer functions $Z$ and $Q$ correspond to the response of the same physical mechanisms probed in small perturbation AC conditions. Such solution can be expressed as a common equivalent circuit (EC), so that the same EC serves to describe IS and IMPS, although with different small amplitude perturbation of the voltage and current sources. In contrast to the description of dye-sensitized solar cells, a widely accepted physical model describing the IS results of PSCs is still unreached. Therefore, independent and simultaneous measuring of IS and IMPS appears as a fruitful strategy for EC model validation. This is why we have developed for the first time the treatment of IMPS at OC, which is the condition where IS of PSCs has been commonly extracted. Our primary aim is to confront EC models and physical parameters extracted by the two kinds of measurements in order to discern about the underlying operating processes.

However, an unexpected result was obtained that reveals significant information. While in principle the same mechanisms are probed by the separate techniques, we show in this paper that in practice IMPS detects additional important components that are hidden in IS spectra. By this methodology, we identify an extra capacitance in $\mathrm{MAPbBr}_{3}$ solar cells at intermediate frequencies (IF) between 1-10 Hz from IMPS measurements that is masked in IS measurements at OC. We obtain that this extra piece of physical information occurs due to the inherent differences in time constants formed in IMPS and IS measurements. We also identify that the resistance associated to this IF capacitor could be a critical component in controlling the total device resistance at low voltages and can hence have a strong impact on the fill factor. In addition, we develop an understanding of the different spectra observed in IMPS measurements and identify that the arcs observed from IMPS measurements are not related to transport times, as commonly interpreted. Finally, using insights from both IMPS and IS measurements, we develop an advanced EC that allows for the interpretation of the small perturbation response of PSCs in general at both OC and SC.

\section{Results and discussion}




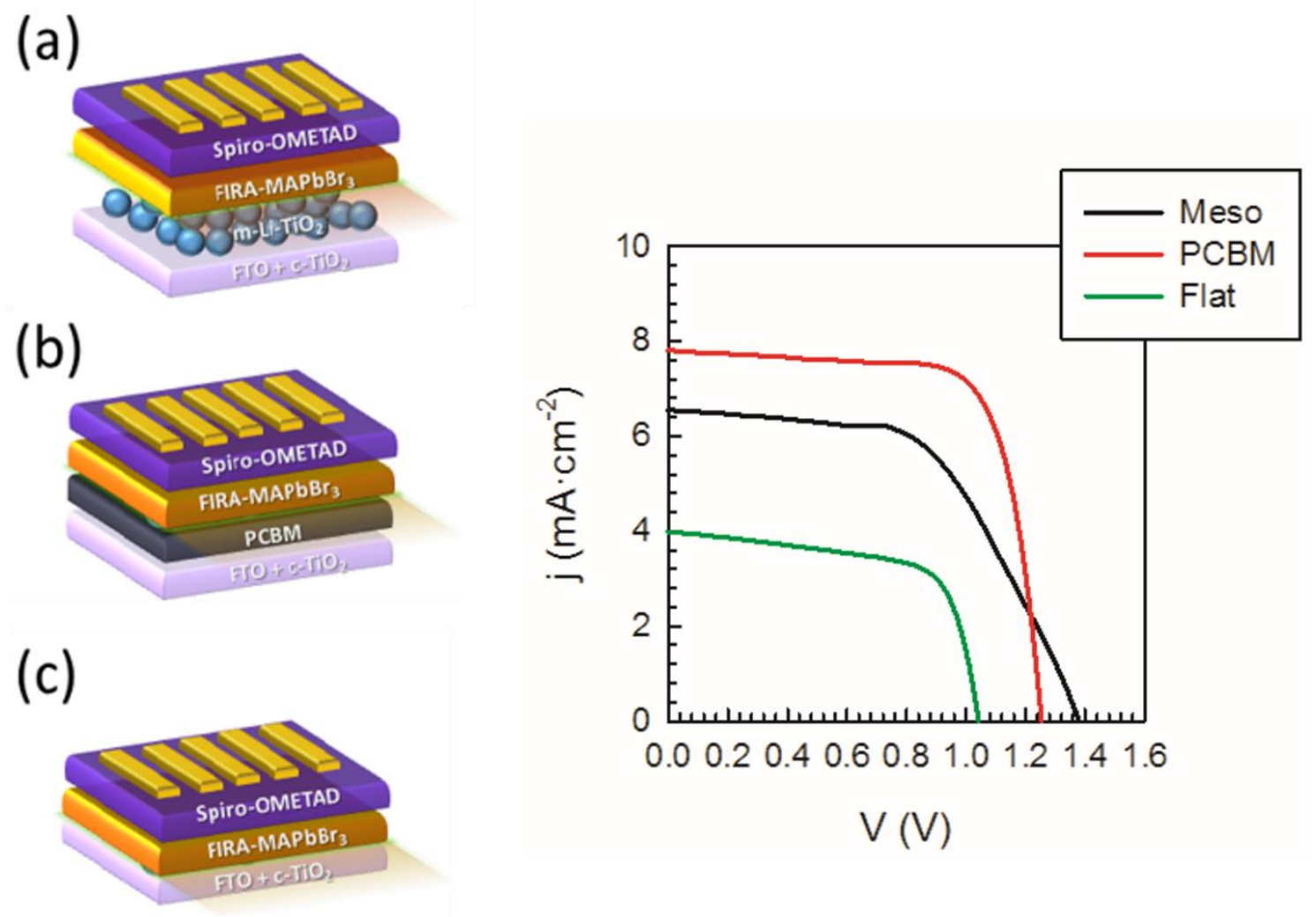

Figure 1 Schematic of measured samples named (a) Meso (b) PCBM and (c) Flat, with corresponding current-voltage curves measured at $40 \mathrm{mV} \cdot \mathrm{s}^{-1}$.

Figure 1 shows the three different $\mathrm{MAPbBr}_{3}$ samples investigated. While each of the samples have a compact $\mathrm{TiO}_{2}\left(\mathrm{c}-\mathrm{TiO}_{2}\right)$ layer, we add a layer of mesoporous- $\mathrm{TiO}_{2}(\mathrm{~m}-$ $\mathrm{TiO}_{2}$ ), PCBM for each sample, respectively named Meso and PCBM. We do not add any layers to the third sample, named Flat ( $a, b$ and $c$ respectively in Figure 1). Details of sample preparation are provided in the SI. The current-voltage $(j-V)$ curves of the samples are shown in Figure 1. One can observe that the addition of a PCBM layer improves the fill factor of the cell strongly compared to the Meso cell. The Flat cell shows a large drop in the short circuit current $\left(j_{s c}\right)$, reflected in the smaller values of the LF intercept on the real axis of IMPS measurements at SC, ${ }^{15}$ shown in Figure SI2. These preliminary results indicate that charge extraction and recombination in our $\mathrm{MAPbBr}_{3}$ cells are strongly dependent on the nature and interactions at the perovskite/ETL interface. Therefore, we focus on IMPS measurements at OC conditions, to identify the different parts in the $\mathrm{MAPbBr}_{3}$ cell architecture that store charge and create extra recombination channels. 
(a)

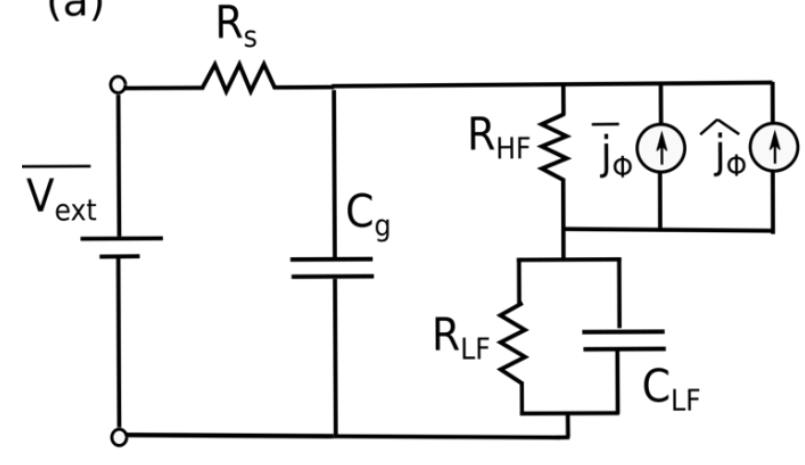

(c)

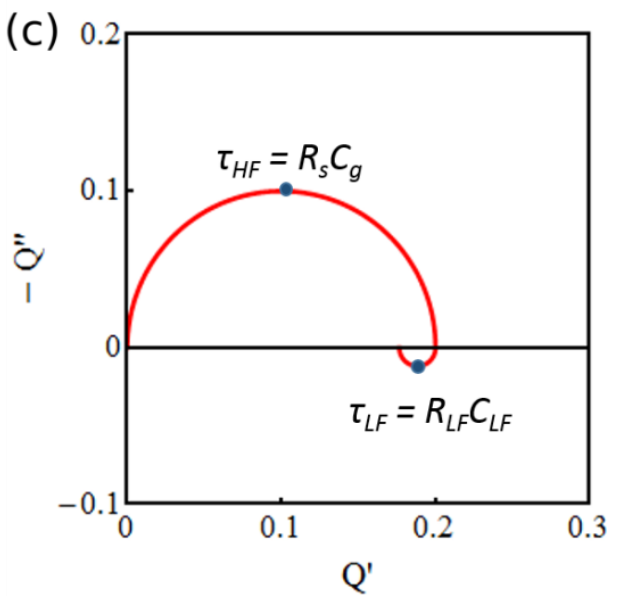

(b)

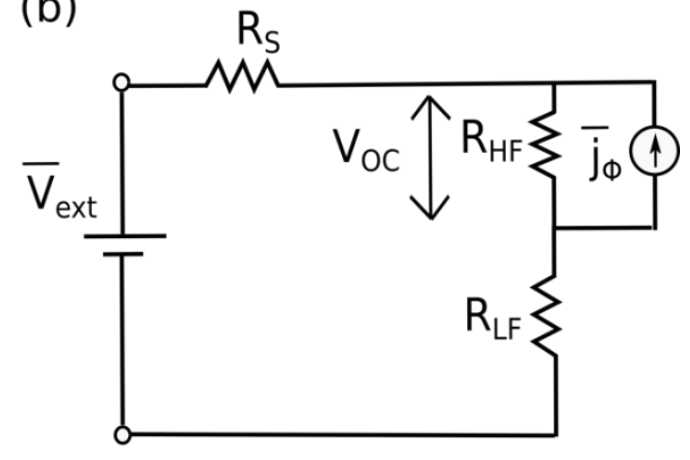

(d)

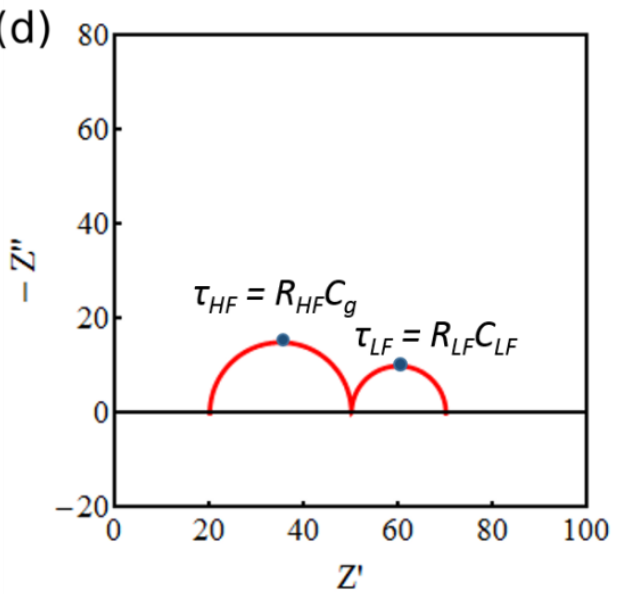

Figure 2 (a) Perovskite solar cell model under an applied modulated photon current density $\hat{j}_{\Phi}$ during an IMPS measurement, containing the external DC driving voltage $\bar{V}_{\text {ext }}$ , steady state photon current density $\overline{j_{\Phi}}$. Circuit elements include a series resistance $R_{s}$ , recombination resistors $R_{H F}$ and $R_{L F}$ respectively, bulk dielectric and interfacial capacitances $C_{g}$ and $C_{L F}$ respectively. (b) DC circuit of (a) at OC conditions (c) Simulated IMPS complex plane plot and (d) corresponding simulated IS complex plane plot. Parameters used were $R_{s}=120$ and $20 \Omega \cdot \mathrm{cm}^{2}$ for (c) and (d) respectively, $R_{H F}=30$ $\Omega \cdot \mathrm{cm}^{2}, R_{L F}=20 \Omega \cdot \mathrm{cm}^{2}, C_{g}=10^{-7} \mathrm{~F} \cdot \mathrm{cm}^{-2}, C_{L F}=10^{-2} \mathrm{~F} \cdot \mathrm{cm}^{-2}$.

Before examining the IMPS measurements of these samples, we first aim to develop a theoretical formulation of the IMPS response for PSCs, starting from the model at SC developed previously, ${ }^{15}$. All steady state quantities are denoted with an overbar and timedependent quantities using a hat. This model is shown in Figure 2a, consisting of the series resistance $R_{s}$, two resistances corresponding to recombination of photogenerated carriers, $R_{H F}$ and $R_{L F}$ respectively. A capacitor $C_{L F}$ is related to interfacial charge accumulation, with the bulk dielectric capacitance $C_{g}$ shunting these elements. The DC current generation term associated to incident photon flux is defined as $\overline{j_{\Phi}}$, represented by a current source. At OC, the circuit includes an external voltage source $\bar{V}_{\text {ext }}$ that opposes the voltage created by photocarrier generation, as seen in Figure $2 b$, ensuring a net zero DC current flowing across the external circuit, yielding the equation 


$$
\overline{V_{e x t}}=\overline{j_{\Phi}} R_{H F}
$$

Upon the application of a modulated photon current density $\hat{j}_{\Phi}$, the modulated extracted current $\hat{j}_{e}$ is then driven through the series resistor $R_{s}$. From basic circuit analysis (see SI for derivation), we obtain $Q$ as

$$
Q(\omega)=\left(1+\frac{R_{s}}{R_{H F}}+i \omega R_{s} C_{g}+\frac{R_{L F}}{R_{H F}}\left[\frac{1+i \omega R_{s} C_{g}}{1+i \omega R_{L F} C_{L F}}\right]\right)^{-1}
$$

The corresponding IS transfer function is given by

$$
Z(\omega)=R_{s}+\left(i \omega C_{g}+\frac{1}{R_{H F}+\left[\frac{1}{R_{L F}}+i \omega C_{L F}\right]^{-1}}\right)^{-1}
$$

\begin{tabular}{|l|l|l|l|l|}
\hline & \multicolumn{1}{|c|}{ IMPS } & Quadrant & IS & Quadrant \\
\hline High frequency & $\tau_{H F}=R_{s} C_{g}$ & Upper & $\tau_{H F}=R_{H F} C_{g}$ & Upper \\
\hline Low frequency & $\tau_{L F}=R_{L F} C_{L F}$ & Lower & $\tau_{L F}=R_{L F} C_{L F}$ & Upper \\
\hline
\end{tabular}

Table 1 Time constants of the different arcs obtained from IMPS and IS measurements and the corresponding quadrants in which they occur in a $Q / Z$-plane plot for the equivalent circuit of Figure 2.

As can be seen from the simulations of Figure $2 \mathrm{c}$ and $2 \mathrm{~d}$, we obtain arcs in the upper and lower $Q$-plane quadrants in an IMPS measurement, whereas two arcs are developed in the upper quadrant of the Z-plane for an IS measurement. The time constants corresponding to these arcs are shown in Table 1. In the case of the IMPS transfer function in Equation 3, it is noted that the IMPS response at SC and OC for a given circuit are identical (see SI and Ref 14). We now proceed to investigate the experimental IMPS and IS response of the $\mathrm{MAPbBr}_{3}$ cells in order to confirm the information developed so far and obtain suitable parameters from the data. 

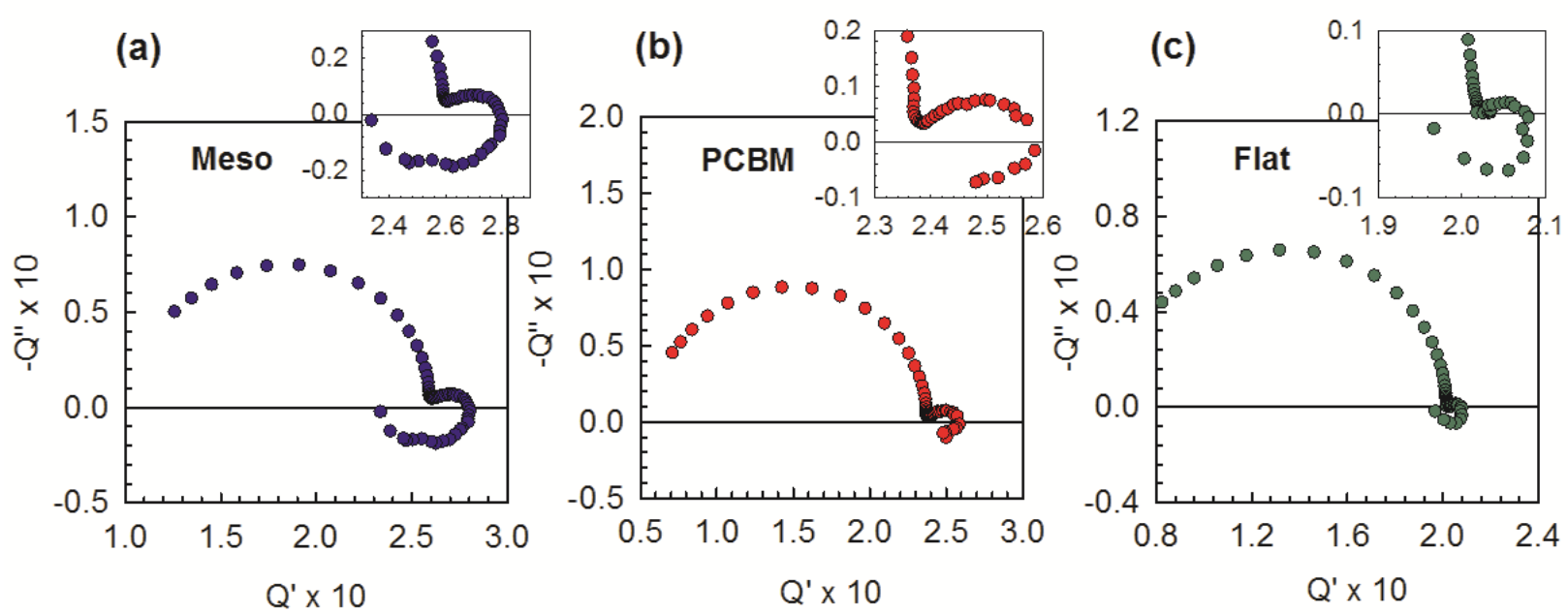

Figure 3 IMPS $Q$-plane plots obtained at OC for illumination at $90 \mathrm{~mW} \cdot \mathrm{cm}^{-2}$ using a blue LED $(470 \mathrm{~nm})$ for different sample configurations as mentioned in the label. Inset shows the intermediate and low frequency arcs.

Figure 3 shows the experimental IMPS Q-plane plot at OC with an illumination intensity of $90 \mathrm{~mW} \cdot \mathrm{cm}^{-2}$ for Meso, Flat and PCBM samples. One can observe a characteristic HF arc in the upper quadrant and a LF arc in the lower quadrant for all samples. As described previously, the time constant of the HF arc is related to the product of $R_{s}$ and the bulk dielectric capacitance $C_{g}$, while the LF arc is the time constant formed by the product of $R_{L F}$ and $C_{L F}$. We note that the values of $Q^{\prime}$ are very low. Since this quantity is related to the external quantum efficiency of the solar cell, ${ }^{12}$ we determined the absorptance of our cells from transmittance and reflectance measurements, shown in Figure SI1(c). The strong absorptance at of all the cells at $470 \mathrm{~nm}$ (blue), the wavelength of input photons in our IMPS measurements, approximately 93\%, indicates that the IMPS transfer function is reflecting the internal quantum efficiency (IQE) of the cells. Our observations indicate poor charge extraction and/or large recombination as expected for OC conditions. The experimental data appears to be similar to the simulation shown in Figure 2c at first glance. 

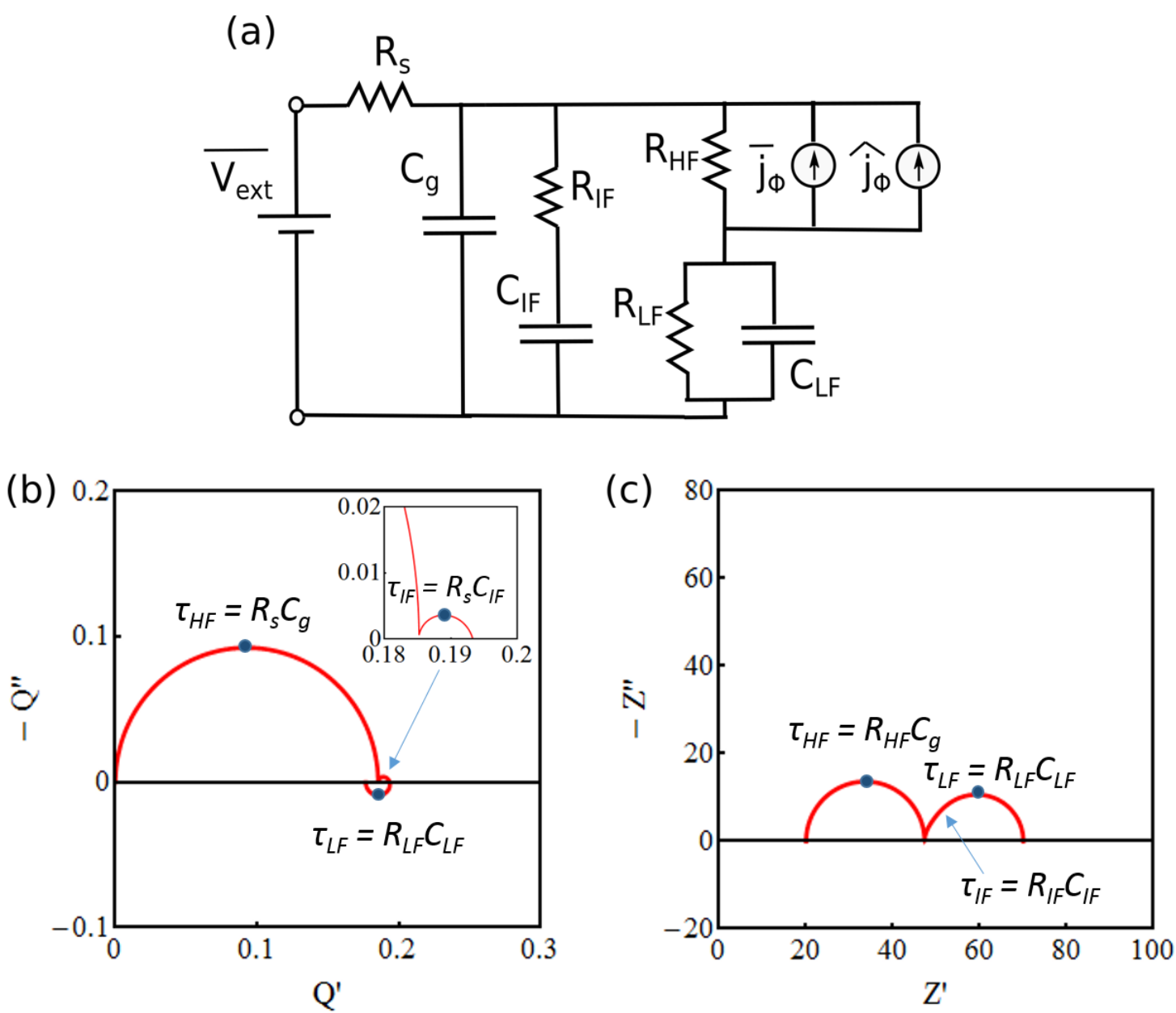

Figure 4 (a) Proposed perovskite solar cell model at OC, with the inclusion of a capacitor $C_{I F}$ that is charged through a resistor $R_{I F}$. (b) Simulated IMPS $Q$-plane plot and (c) IS $Z$-plane plot at OC using circuit of (a). Parameters used were $R_{s}=120$ and 20 $\Omega \cdot \mathrm{cm}^{2}$ for (b) and (c) respectively, $R_{H F}=30 \Omega \cdot \mathrm{cm}^{2}, R_{L F}=20 \Omega \cdot \mathrm{cm}^{2}, R_{I F}=300 \Omega \cdot \mathrm{cm}^{2}$, $C_{g}=10^{-7} \mathrm{~F} \cdot \mathrm{cm}^{-2}, C_{I F}=10^{-4} \mathrm{~F} \cdot \mathrm{cm}^{-2}, C_{L F}=10^{-2} \mathrm{~F} \cdot \mathrm{cm}^{-2}$.

However, from the insets of Figure 3, we can see a small intermediate frequency (IF) arc in the upper quadrant which is slightly masked by the LF data for PCBM and Flat cells, but is well resolved for Meso cells, with a corresponding characteristic frequency between 1-10 Hz. In order to confirm the existence of this extra arc, we turn to the IMPS measurements at SC, shown in Figure SI2. The IF arc is very well resolved and dominates the spectra at SC. Therefore, in order to account for this IF process, we expand the basic IMPS model of the PSC by the addition of a capacitor $C_{I F}$, with a corresponding resistor $R_{I F}$ that controls the charging kinetics of this capacitor. An $R_{I F} C_{I F}$ line is therefore added to the equivalent circuit of Figure 2 (see Figure 4a). From basic circuit analysis, the IMPS transfer function is calculated (see SI for derivation) and one can simulate its IMPS (see Figure 4b) and IS complex plane plot (see Figure 4c). Figure 4b indeed yields the experimentally observed three arcs of Figure 3 of IMPS. We find that the IF arc in the 
upper quadrant corresponds to a time constant formed between $R_{s}$ and $C_{I F}$ (see SI). Therefore, it is found that arcs in the upper quadrant of an IMPS spectrum are time constants formed between the series resistance and capacitors in associated lines of the circuit and the LF arc in the lower quadrant is related to the time constant formed by the low frequency capacitor and resistor due to the internal coupling of charge generation with $R_{H F} \cdot{ }^{15}$ Hence, these time constants are not related to any transport processes as is commonly interpreted.

\begin{tabular}{|l|l|l|l|l|l|l|l|l|}
\hline & $\begin{array}{l}R_{s} \\
\left(\Omega \cdot \mathrm{cm}^{2}\right)\end{array}$ & $\begin{array}{l}R_{H F} \\
\left(\Omega \cdot \mathrm{cm}^{2}\right)\end{array}$ & $\begin{array}{l}R_{L F} \\
\left(\Omega \cdot \mathrm{cm}^{2}\right)\end{array}$ & $\begin{array}{l}R_{I F} \\
\left(\Omega \cdot \mathrm{cm}^{2}\right)\end{array}$ & $\begin{array}{l}C_{g, I M P S} \\
\left(\mathrm{~F} \cdot \mathrm{cm}^{-2}\right)\end{array}$ & $\begin{array}{l}C_{g, I S} \\
\left(\mathrm{~F} \cdot \mathrm{cm}^{-2}\right)\end{array}$ & $\begin{array}{l}C_{I F} \\
\left(\mathrm{~F} \cdot \mathrm{cm}^{-2}\right)\end{array}$ & $\begin{array}{l}C_{L F} \\
\left(\mathrm{~F} \cdot \mathrm{cm}^{-2}\right)\end{array}$ \\
\hline Meso & 95 & 37 & 26 & 366 & $2.6 \times 10^{-7}$ & $2 \times 10^{-7}$ & $1.3 \times 10^{-4}$ & $4.8 \times 10^{-2}$ \\
\hline Flat & 125 & 33 & 9 & 994 & $2.0 \times 10^{-7}$ & $4 \times 10^{-7}$ & $8.0 \times 10^{-4}$ & $7.0 \times 10^{-2}$ \\
\hline PCBM & 125 & 43 & 11 & 392 & $2.5 \times 10^{-7}$ & $3 \times 10^{-7}$ & $8.0 \times 10^{-4}$ & $1.8 \times 10^{-1}$ \\
\hline
\end{tabular}

Table 2 Extracted parameters from IMPS and IS for different samples

With respect to the IS simulations, we find that Figure 4c also yields 2 arcs, similar to Figure $2 \mathrm{~d}$, with the only difference being a slight modification at intermediate frequencies that slightly deforms the LF arc. This is also observed in the measured IS spectra shown in Figure SI1. This masking effect in IS measurements occurs because the IF time constant is formed by the product of $R_{I F}$ and $C_{I F}$ (see $\mathrm{SI}$ ), whose value is similar to that of the LF time constant formed by $R_{L F}$ and $C_{L F}$, as we confirm subsequently. Hence, in IS measurements, the IF and LF arcs are difficult to separate at OC conditions, but IMPS measurements at OC exhibit a clearly visible IF arc because the time constant formed is between $R_{s}$ and $C_{I F}$, whose value is smaller than the IF time constant of IS measurements. In addition, IF features in IMPS measurements are moved to the lower quadrant, making it distinguishible.

Using the experimental IMPS spectra, Equation 2 and parameter definitions in SI, we can estimate the values of the different capacitances and resistances in the circuit, which are shown in Table 2. Also, IS measurements at OC (see Figure SI1) are measured in order to corroborate the value of $C_{g}$ obtained from IMPS. As seen from Table 2, the values of $C_{g}$ extracted from IMPS and IS are in good agreement with each other. The values of $C_{L F}$ are quite large, in the order of $\mathrm{mF}$, similar to that of the LF capacitance usually observed from IS. ${ }^{24}$ Keeping in mind that the $\mathrm{TiO}_{2} /$ perovskite interface has also been proven to be the predominant recombination channel in Br-based perovskite solar cells ${ }^{25}$ and the $\mathrm{Br}$ ion is smaller than the I ion, we can attribute this large LF capacitance and its associated resistance to the accumulation of ionic and electronic carriers at the c$\mathrm{TiO}_{2} /$ perovskite interface ${ }^{23}$ and the subsequent recombination of the electronic carriers at this interface. ${ }^{26-27}$ The IF capacitance is of the order of $10^{-4} \mathrm{~F} \cdot \mathrm{cm}^{-2}$ for all the cells, two orders smaller than the LF capacitance. We therefore attribute this capacitance to the accumulation of anions at the perovskite/Spiro-OMeTAD interface. 


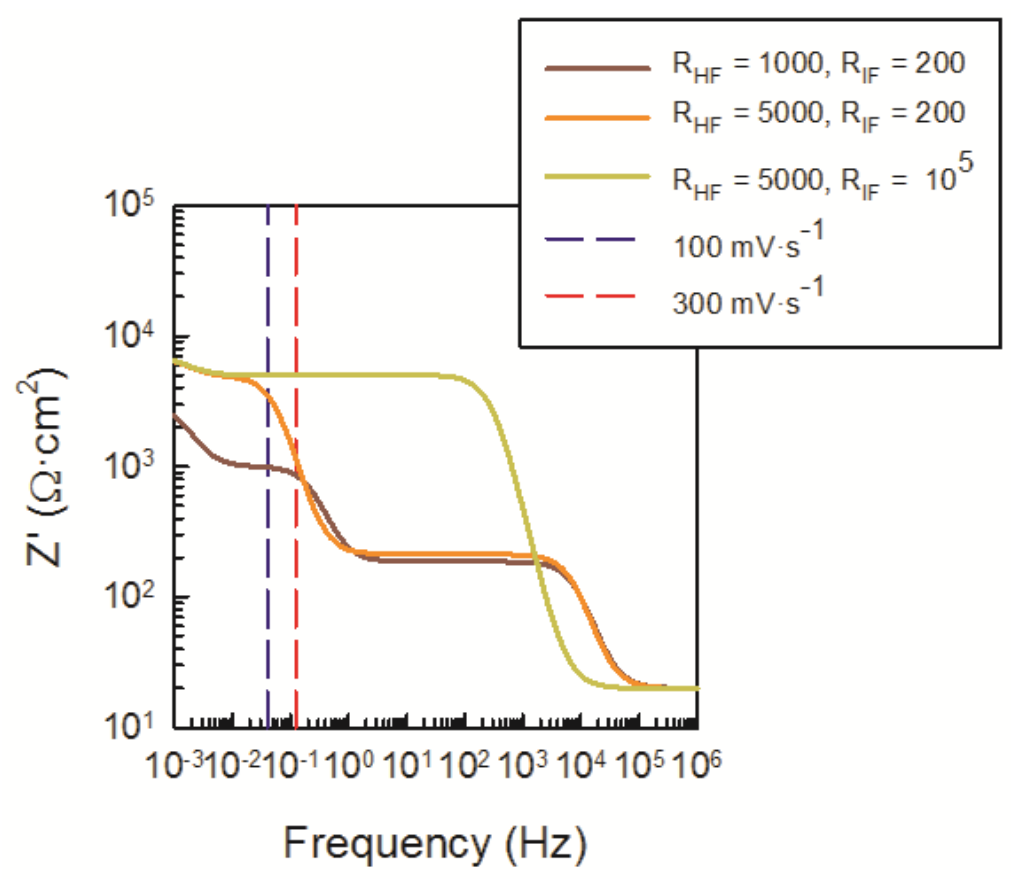

Figure 5 Simulated real part of resistance values versus frequency using the equivalent circuit of Figure 4(a), for different values of $R_{H F}$ and $R_{I F}$ as shown in the legend. The dotted lines indicate the frequencies corresponding to scan rates of $100 \mathrm{mV} \cdot \mathrm{s}^{-1}$ (blue) and $300 \mathrm{mV} \cdot \mathrm{s}^{-1}$ (red) respectively. Parameters used were $R_{s}=20 \Omega \cdot \mathrm{cm}^{2}, R_{L F}=2000 \Omega \cdot \mathrm{cm}^{2}$, $C_{g}=10^{-7} \mathrm{~F} \cdot \mathrm{cm}^{-2}, C_{I F}=5 \cdot 10^{-4} \mathrm{~F} \cdot \mathrm{cm}^{-2}, C_{L F}=5 \cdot 10^{-2} \mathrm{~F} \cdot \mathrm{cm}^{-2}$.

The large values of this capacitance indicate that the stored charge at hole extracting interface could have an electronic component as well, indicating a strong downward band bending on the perovskite side at the perovskite/HTL interface. This effect has been observed previously in the absence of $\mathrm{TiO}_{2}$ as the ETL with different HTL layers. ${ }^{28}$ The variation of this capacitance with the ETL is due to the fact the ETL/perovskite interface capacitance has been shown ${ }^{29}$ to strongly affect the nature of the electrical potential distribution throughout the device, especially within the hole transport layer (HTL), which affects the density of charge and hence the capacitance at this interface. Therefore, it is expected that any change in the ETL/perovskite interface should influence the potential distribution within the device and hence alter the charge densities and capacitance at the perovskite/HTL interface. The $R_{I F}$ values become significant at lower voltages, where $R_{H F}$ and $R_{L F}$, which are recombination resistors, become very large. In such cases, $R_{I F}$ will be the dominant resistance since it is in parallel to the HF and LF resistors. Now a question appears, why should $R_{I F}$ be relevant to the steady state operation of the solar cell if it has a capacitor in series? This $R C$ series branch of the EC should consequently disappear.

However one needs to remember that the $j-V$ curve is actually measured at a certain scan rate, however low it may be. In the simulations of Figure 5, it is observed that the 
resistance at standard scan rates for a $j-V$ curve between $100-300 \mathrm{mV} \cdot \mathrm{s}^{-1}$ is lowered due to the IF resistance, whose absence otherwise yields a constant resistance over a large range of frequencies. Therefore, we propose that the lower values of $R_{I F}$ for the Meso cell leads to its reduced fill factor as seen in Figure 1, compared to the fill factors of PCBM and Flat cells, which have larger $R_{I F}$ values. This finding will be very important in understanding the variations in fill factor in a $j-V$ curve for perovskite solar cells, especially the large drop in fill factor when scanning from SC to OC, which is yet to be understood. ${ }^{30}$

We also note that IMPS measurements at OC yield apparent large series resistance values, as seen in Table 2 . Instead of $R_{s} \approx 10-20 \Omega \cdot \mathrm{cm}^{2}$ as usually derived from IS analysis, much larger values approximately equal to $R_{s} \approx 100 \Omega \cdot \mathrm{cm}^{2}$ are obtained. Therefore, it must be considered that there is an extra resistor in series with $R_{s}$, which can be a transport resistance $R_{t}\left(\Omega \cdot \mathrm{cm}^{2}\right)$ that depends on the conductivity $\sigma$ of the sample. This is given by $^{31}$

$$
\begin{aligned}
& R_{t}=\frac{l}{\sigma} \\
& \sigma=p q \mu_{p}
\end{aligned}
$$

where $l$ is the sample thickness, $p$ is the carrier density, $q$ is the electronic charge and $\mu_{p}$ is the hole mobility. Taking an intermediate value of hole mobility as $40 \mathrm{~cm}^{2} \mathrm{~V}^{-1} \mathrm{~s}^{-1}$ from reported values ${ }^{32-34}$ for $\mathrm{MAPbBr}_{3}$ solar cells between 9-115 $\mathrm{cm}^{2} \mathrm{~V}^{-1} \mathrm{~s}^{-1}$, and estimating a hole density of $2 \times 10^{16} \mathrm{~cm}^{-3}$ corresponding to a irradiation intensity of 90 $\mathrm{mW} \cdot \mathrm{cm}^{-2}$ of $470 \mathrm{~nm}$ blue photons impinging on the sample during an IMPS measurement and total absorption, we obtain a transport resistance value of $\sim 2.3 \times 10^{-4} \Omega \cdot \mathrm{cm}^{2}$ for a 300 $\mathrm{nm}$ thick sample, which is negligibly small. This discrepancy leads us to consider that the proposed EC in Figure 4a need to be modified by including an extra parallel resistance that is apparently invisible at OC conditions as the recombination resistances dominate. Its effect is added to the series resistance in the current EC. Therefore, it is likely that an extra resistor in parallel becomes significant at SC and lowers the charge extraction at SC (as observed from Figure SI2). However, the placement of this resistor in the EC is a nontrivial task that requires combining inferences of IMPS with other techniques such as Kelvin force probe measurements to visualize the charge densities at OC and SC conditions, which is out of the scope of this paper.

Our analysis shows that the direct association of time constants to spectral features, which is a frequently adopted procedure in this field, makes it difficult and sometimes impossible to confront the results extracted from different experimental methods. This occurs since the apparent time constants correspond to the coupled combination of different circuit elements in each method, indicating that the physical meaning of time constants is rather distinct and depends on specific measuring assumptions. Therefore, we show the importance of establishing the underlying equivalent circuit model composed of resistances and capacitors that provides an irreducible description of the spectral data obtained separately by different methods. Subsequent work, which is out of the scope of 
this paper, will involve development of the understanding of the recombination processes at SC using IMPS to further improve the EC.

\section{Conclusions}

We have carried out Intensity Modulated Photocurrent Spectroscopy measurements at open circuit conditions on $\mathrm{CH}_{3} \mathrm{NH}_{3} \mathrm{PbBr}_{3}$ solar cells with different electron selective contact layers. From these measurements, we identify the location of the dominant recombination channel at the $\mathrm{TiO}_{2} /$ perovskite interface, associated with a large low frequency capacitance. We also uncover an extra capacitance of the order of $10^{-4} \mathrm{~F} \cdot \mathrm{cm}^{-2}$, which we associate with the accumulation of anions at the perovskite/SpiroOMeTAD interface at open circuit conditions that likely also includes an electronic component. This capacitance is observed at frequencies between $1-10 \mathrm{~Hz}$ and is not observable from Impedance Spectroscopy measurements. The recombination associated to this capacitor is dominant at low voltages and hence will be important in understanding carrier losses and fill factor variations. Our results establish a robust equivalent circuit for the analysis of perovskite solar cells by the combination of different small perturbation frequency techniques.

\section{Acknowledgements}

We thank financial support by MINECO of Spain under project (MAT2016-76892C3-1-R). Universitat Jaume I is also acknowledged for financial support (UJI-B2017-32).

\section{For Table of Contents Only}

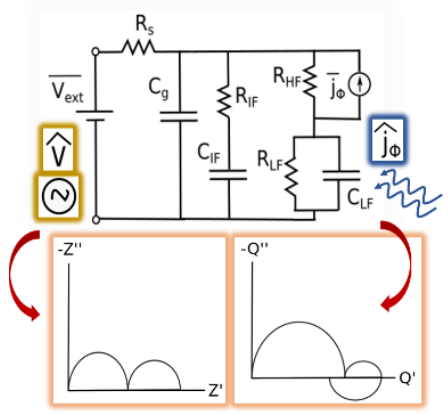




\section{References}

1. Li, C.; Guerrero, A.; Huettner, S.; Bisquert, J. Unravelling the role of vacancies in lead halide perovskite through electrical switching of photoluminescence. Nature Communications 2018, 9 (1), 5113.

2. Li, C.; Guerrero, A.; Zhong, Y.; Gräser, A.; Luna, C. A. M.; Köhler, J.; Bisquert, J.; Hildner, R.; Huettner, S. Real-Time Observation of Iodide Ion Migration in Methylammonium Lead Halide Perovskites. Small 2017, 13 (42), 1701711.

3. Zarazúa, I.; Sidhik, S.; Lopéz-Luke, T.; Esparza, D.; De la Rosa, E.; ReyesGomez, J.; Mora-Seró, I.; Garcia-Belmonte, G. Operating Mechanisms of Mesoscopic Perovskite Solar Cells through Impedance Spectroscopy and J-V Modeling. The Journal of Physical Chemistry Letters 2017, 8 (24), 6073-6079.

4. Zarazua, I.; Han, G.; Boix, P. P.; Mhaisalkar, S.; Fabregat-Santiago, F.; MoraSeró, I.; Bisquert, J.; Garcia-Belmonte, G. Surface Recombination and Collection Efficiency in Perovskite Solar Cells from Impedance Analysis. The Journal of Physical Chemistry Letters 2016, 7 (24), 5105-5113.

5. Almora, O.; Cho, K. T.; Aghazada, S.; Zimmermann, I.; Matt, G. J.; Brabec, C. J.; Nazeeruddin, M. K.; Garcia-Belmonte, G. Discerning recombination mechanisms and ideality factors through impedance analysis of high-efficiency perovskite solar cells. Nano Energy 2018, 48, 63-72.

6. Guerrero, A.; Garcia-Belmonte, G.; Mora-Sero, I.; Bisquert, J.; Kang, Y. S.; Jacobsson, T. J.; Correa-Baena, J.-P.; Hagfeldt, A. Properties of Contact and Bulk Impedances in Hybrid Lead Halide Perovskite Solar Cells Including Inductive Loop Elements. The Journal of Physical Chemistry C 2016, 120 (15), 8023-8032.

7. Fabregat-Santiago, F.; Kulbak, M.; Zohar, A.; Vallés-Pelarda, M.; Hodes, G.; Cahen, D.; Mora-Seró, I. Deleterious Effect of Negative Capacitance on the Performance of Halide Perovskite Solar Cells. ACS Energy Letters 2017, 2 (9), 2007-2013.

8. Almora, O.; Zarazua, I.; Mas-Marza, E.; Mora-Sero, I.; Bisquert, J.; GarciaBelmonte, G. Capacitive Dark Currents, Hysteresis, and Electrode Polarization in Lead Halide Perovskite Solar Cells. The Journal of Physical Chemistry Letters 2015, 6 (9), 1645-1652.

9. Pitarch-Tena, D.; Ngo, T. T.; Vallés-Pelarda, M.; Pauporté, T.; Mora-Seró, I. Impedance Spectroscopy Measurements in Perovskite Solar Cells: Device Stability and Noise Reduction. ACS Energy Letters 2018, 3 (4), 1044-1048.

10. Troughton, J.; Carnie, M. J.; Davies, M. L.; Charbonneau, C.; Jewell, E. H.; Worsley, D. A.; Watson, T. M. Photonic flash-annealing of lead halide perovskite solar cells in 1 ms. Journal of Materials Chemistry A 2016, 4 (9), 3471-3476.

11. Sanchez, S.; Christoph, N.; Grobety, B.; Phung, N.; Steiner, U.; Saliba, M.; Abate, 
A. Efficient and Stable Inorganic Perovskite Solar Cells Manufactured by Pulsed Flash Infrared Annealing. Advanced Energy Materials 2018, 8 (30), 1802060.

12. Bertoluzzi, L.; Bisquert, J. Investigating the consistency of models for water splitting systems by light and voltage modulated techniques. The Journal of Physical Chemistry Letters 2016, 8 (1), 172-180.

13. Peter, L. M. Dynamic aspects of semiconductor photoelectrochemistry. Chemical Reviews 1990, 90 (5), 753-769.

14. Pockett, A.; Eperon, G. E.; Peltola, T.; Snaith, H. J.; Walker, A.; Peter, L. M.; Cameron, P. J. Characterization of planar lead halide perovskite solar cells by impedance spectroscopy, open-circuit photovoltage decay, and intensity-modulated photovoltage/photocurrent spectroscopy. The Journal of Physical Chemistry C 2015, 119 (7), 3456-3465.

15. Ravishankar, S.; Aranda, C.; Boix, P. P.; Anta, J. A.; Bisquert, J.; GarciaBelmonte, G. Effects of Frequency Dependence of the External Quantum Efficiency of Perovskite Solar Cells. The Journal of Physical Chemistry Letters 2018, 9 (11), 30993104

16. Domanski, K.; Roose, B.; Matsui, T.; Saliba, M.; Turren-Cruz, S.-H.; CorreaBaena, J.-P.; Carmona, C. R.; Richardson, G.; Foster, J. M.; De Angelis, F. Migration of cations induces reversible performance losses over day/night cycling in perovskite solar cells. Energy \& Environmental Science 2017, 10 (2), 604-613.

17. Guillén, E.; Ramos, F. J.; Anta, J. A.; Ahmad, S. Elucidating TransportRecombination Mechanisms in Perovskite Solar Cells by Small-Perturbation Techniques. The Journal of Physical Chemistry C 2014, 118 (40), 22913-22922.

18. Todinova, A.; Idígoras, J.; Salado, M.; Kazim, S.; Anta, J. A. Universal Features of Electron Dynamics in Solar Cells with $\mathrm{TiO}_{2}$ Contact: From Dye Solar Cells to Perovskite Solar Cells. The Journal of Physical Chemistry Letters 2015, 6 (19), 39233930.

19. Correa-Baena, J.-P.; Anaya, M.; Lozano, G.; Tress, W.; Domanski, K.; Saliba, M.; Matsui, T.; Jacobsson, T. J.; Calvo, M. E.; Abate, A.; Grätzel, M.; Míguez, H.; Hagfeldt, A. Unbroken Perovskite: Interplay of Morphology, Electro-optical Properties, and Ionic Movement. Advanced Materials 2016, 28 (25), 5031-5037.

20. Contreras, L.; Idigoras, J.; Todinova, A.; Salado, M.; Kazim, S.; Ahmad, S.; Anta, J. A. Specific cation interactions as the cause of slow dynamics and hysteresis in dye and perovskite solar cells: a small-perturbation study. Physical Chemistry Chemical Physics 2016, 18 (45), 31033-31042.

21. Ponomarev, E.; Peter, L. A generalized theory of intensity modulated photocurrent spectroscopy (IMPS). Journal of Electroanalytical Chemistry 1995, 396 (1-2), 219-226. 
22. Bisquert, J. Theory of the impedance of electron diffusion and recombination in a thin layer. The Journal of Physical Chemistry B 2002, 106 (2), 325-333.

23. Ravishankar, S.; Gharibzadeh, S.; Roldán-Carmona, C.; Grancini, G.; Lee, Y.; Ralaiarisoa, M.; Asiri, A. M.; Koch, N.; Bisquert, J.; Nazeeruddin, M. K. Influence of Charge Transport Layers on Open-Circuit Voltage and Hysteresis in Perovskite Solar Cells. Joule 2018, 2 (4), 788-798.

24. Zarazua, I.; Bisquert, J.; Garcia-Belmonte, G. Light-induced space-charge accumulation zone as photovoltaic mechanism in perovskite solar cells. The Journal of Physical Chemistry Letters 2016, 7 (3), 525-528.

25. Zohar, A.; Kulbak, M.; Levine, I.; Hodes, G.; Kahn, A.; Cahen, D. What Limits the Open-Circuit Voltage of Bromide Perovskite-Based Solar Cells? ACS Energy Letters 2018, 1-7.

26. Ravishankar, S.; Almora, O.; Echeverría-Arrondo, C.; Ghahremanirad, E.; Aranda, C.; Guerrero, A.; Fabregat-Santiago, F.; Zaban, A.; Garcia-Belmonte, G.; Bisquert, J. Surface Polarization Model for the Dynamic Hysteresis of Perovskite Solar Cells. The Journal of Physical Chemistry Letters 2017, 8 (5), 915-921.

27. Gottesman, R.; Lopez-Varo, P.; Gouda, L.; Jimenez-Tejada, J. A.; Hu, J.; Tirosh, S.; Zaban, A.; Bisquert, J. Dynamic Phenomena at Perovskite/Electron-Selective Contact Interface as Interpreted from Photovoltage Decays. Chem 2016, 1 (5), 776-789.

28. Guerrero, A.; Bou, A.; Matt, G.; Almora, O.; Heumüller, T.; Garcia-Belmonte, G.; Bisquert, J.; Hou, Y.; Brabec, C. Switching Off Hysteresis in Perovskite Solar Cells by Fine-Tuning Energy Levels of Extraction Layers. Advanced Energy Materials 2018, 8 (21), 1703376.

29. Hermes, I. M.; Hou, Y.; Bergmann, V. W.; Brabec, C. J.; Weber, S. A. The Interplay of Contact Layers: How the Electron Transport Layer Influences Interfacial Recombination and Hole Extraction in Perovskite Solar Cells. The Journal of Physical Chemistry Letters 2018, 9 (21), 6249-6256.

30. Lopez-Varo, P.; Jiménez-Tejada, J. A.; García-Rosell, M.; Ravishankar, S.; Garcia-Belmonte, G.; Bisquert, J.; Almora, O. Device physics of hybrid perovskite solar cells: theory and experiment. Advanced Energy Materials 2018, 8 (14), 1702772.

31. Bisquert, J. The Physics of Solar Cells: Perovskites, Organics, and Photovoltaic Fundamentals. CRC Press: 2017.

32. Shi, D.; Adinolfi, V.; Comin, R.; Yuan, M.; Alarousu, E.; Buin, A.; Chen, Y.; Hoogland, S.; Rothenberger, A.; Katsiev, K.; Losovyj, Y.; Zhang, X.; Dowben, P. A.; Mohammed, O. F.; Sargent, E. H.; Bakr, O. M. Low trap-state density and long carrier diffusion in organolead trihalide perovskite single crystals. Science 2015, 347 (6221), 519.

33. Saidaminov, M. I.; Abdelhady, A. L.; Murali, B.; Alarousu, E.; Burlakov, V. M.; 
Peng, W.; Dursun, I.; Wang, L.; He, Y.; Maculan, G.; Goriely, A.; Wu, T.; Mohammed, O. F.; Bakr, O. M. High-quality bulk hybrid perovskite single crystals within minutes by inverse temperature crystallization. Nature Communications 2015, 6, 7586.

34. Sheng, R.; Ho-Baillie, A.; Huang, S.; Chen, S.; Wen, X.; Hao, X.; Green, M. A. Methylammonium Lead Bromide Perovskite-Based Solar Cells by Vapor-Assisted Deposition. The Journal of Physical Chemistry C 2015, 119 (7), 3545-3549. 


\title{
Supporting Information for
}

\section{Perovskite Solar Cell Modeling using Light and Voltage Modulated Techniques}

\author{
Sandheep Ravishankar, ${ }^{1}$ Clara Aranda, ${ }^{1}$ Sandy Sanchez, ${ }^{2}$ Juan Bisquert, ${ }^{1}$ \\ Michael Saliba, ${ }^{2^{*}}$ and Germà Garcia-Belmonte ${ }^{1^{*}}$ \\ ${ }^{1}$ Institute of Advanced Materials (INAM), Universitat Jaume I, 12006 Castelló, Spain \\ ${ }^{2}$ Adolphe Merkle Institute, Chemin des Verdiers 4, CH-1700, Fribourg, Switzerland
}

\section{Experimental details}

The devices were prepared similar to previously published work. ${ }^{1}$ In brief:

Preparation of substrate:

Prior to the deposition of $\mathrm{TiO}_{2}$ compact layer, the FTO substrate was partially etched with zinc powder and $\mathrm{HCl}(2 \mathrm{M})$ and cleaned by sonication in Hellmanex detergent, rinsed with Milli-Q water followed by sonication in ethanol and isopropanol. After that, the substrates were treated with plasma oxygen for $10 \mathrm{~min}$.

$\underline{\text { Compact layer } \mathrm{TiO}_{2}}$

The $\mathrm{TiO}_{2}$ blocking layer was deposited by aerosol spray pyrolysis at $450{ }^{\circ} \mathrm{C}$, using a commercial titanium diisopropoxide bis(acetylacetonate) solution (75\% in 2-propanol, Sigma-Aldrich) diluted in ethanol $(1: 9, \mathrm{v} / \mathrm{v})$ as precursor, with oxygen as carrier gas spraying a total volume of $10 \mathrm{~mL}$.

PCBM layer

Onto the $\mathrm{TiO}_{2}$ blocking layer, $50 \mu \mathrm{l}$ of a $20 \mathrm{mg} / \mathrm{ml}$ solution of PCBM in chloroform was deposited by spin coating and sintering at $90^{\circ} \mathrm{C}$ for 20 min under glovebox atmosphere.

$\underline{\text { Mesoporous layer } \mathrm{TiO}_{2}}$

To form the mesoporous layer, diluted paste of $\mathrm{TiO}_{2}$ (1:5, weight ratio) (Dyesol 30NRD: ethanol) was spin-coated and sintered at $450^{\circ} \mathrm{C}$ for $30 \mathrm{~min}$ at ambient atmosphere.

Li-TFSI treatment

Before the deposition of perovskite films, $100 \mu \mathrm{l}$ of a lithium bis(trifluoromethylsulfonyl) imide $35 \mathrm{mM}$ solution in acetonitrile, was prepared and deposited on the top of the device by spin-coating, followed by sintering at $450^{\circ} \mathrm{C}$ for 30 $\min$.

Deposition of $\mathrm{MAPbBr}_{3}$ perovskite material: 
All materials were purchased from Sigma-Aldrich and TCI and were used as received. $\mathrm{MAPbBr}_{3}$ film was deposited, at room atmosphere, using one-step deposition method. $1.4 \mathrm{M}$ perovskite solution in N,Ndimethylformamide (DMF) and dimethylsulphoxide (DMSO) (solvent ratio 3:1) was prepared. $\mathrm{MAPbBr}_{3}$ solution was deposited by spin coating method at $4000 \mathrm{rpm}$ for $10 \mathrm{~s}$, followed by FIRA method.

Deposition of hole transporting material and device fabrication:

To complete the fabrication of device, a solution of Spiro-OMeTAD as HTM, was prepared by dissolving $72.3 \mathrm{mg}$ of $\left(2,2^{\prime}, 7,7^{\prime}\right.$-tetrakis(N,N'-di-pmethoxyphenylamine)9,9'-spirobifluorene) in $1 \mathrm{~mL}$ of chlorobenzene, $28.8 \mu \mathrm{L}$ of 4-tert-butylpyridine and 17.5 $\mu \mathrm{L}$ of a stock solution of $520 \mathrm{mg} / \mathrm{mL}$ of lithium bis- (trifluoromethylsulfonyl) imide in acetonitrile, as additives. The perovskite film was then covered with $50 \mu \mathrm{L}$ of the HTM solution by dynamically spin coating at $4000 \mathrm{rpm}$ for $20 \mathrm{~s}$. Finally, $80 \mathrm{~nm}$ of gold was thermally evaporated on the top of the device as a back contact, using a commercial Univex 250 chamber, from Oerlikon Leybold Vacuum.

\section{IMPS and IS measurements}

IMPS measurements were carried out at OC conditions, with the DC voltage bias determined from chronopotentiometry measurements for 60 seconds at the given DC light illumination. These measurements were made using a blue (470 nm) LED supplied by Philips LUMILEDS, and the distance between the sample and LED was adjusted to obtain the range of DC bias light intensities necessary, calibrated using a Si photodiode. The LED was driven using an Autolab LED driver through an Autolab potentiostat. In all cases, the perturbation was $10 \%$ of the DC bias light intensity. The range of frequencies investigated was $20 \mathrm{kHz}-10 \mathrm{mHz}$. All IS measurements were carried out OC conditions under 1 sun illumination with a perturbing voltage of $20 \mathrm{mV}$ and scanned over the frequency range $1 \mathrm{MHz}-20 \mathrm{mHz}$.

\section{Absorption measurements}

Transmittance (\%T) and reflectance (\%R) measurements were made using a Cary UVvis spectrophotometer scanned from $300-900 \mathrm{~nm}$. The percentage absorptance (\% A) was calculated as $\% \mathrm{~A}=100-(\% \mathrm{~T}+\% \mathrm{R})$. 

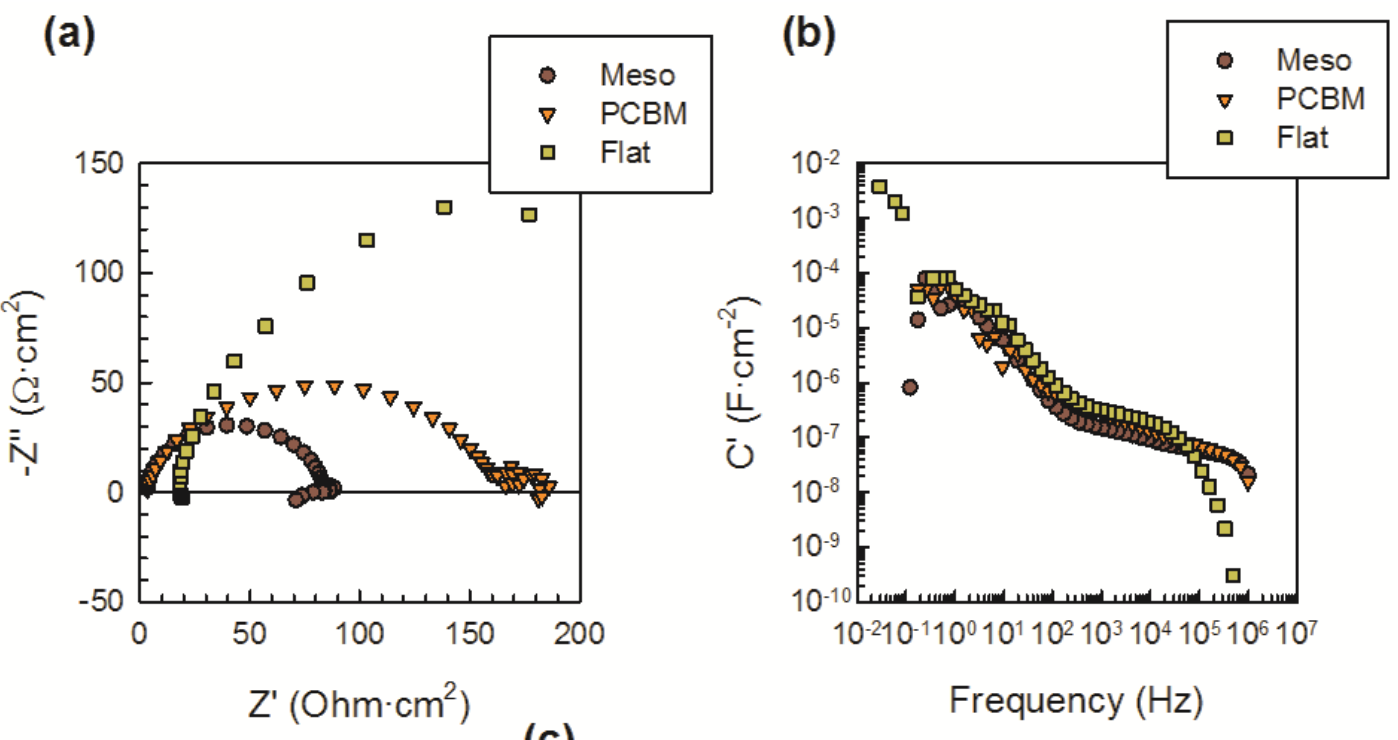

(c)

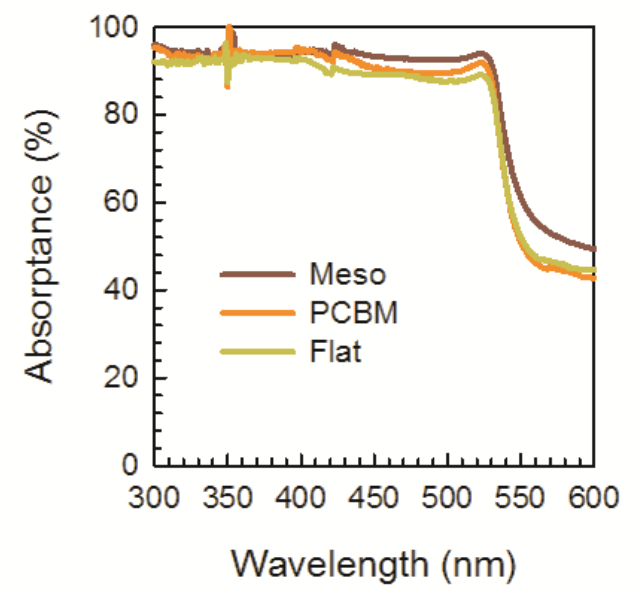

Figure SI1 (a) IS Z-plane plots and (b) corresponding real part of capacitance obtained from IS measurements at OC at 1 sun illumination. (c) Absorptance of samples obtained from transmittance and reflectance measurements. 


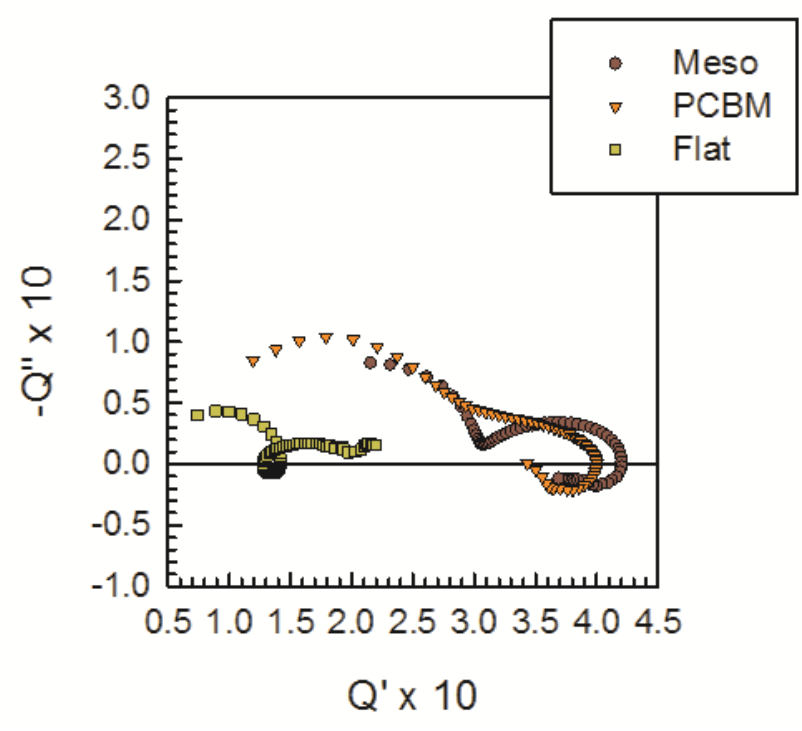

Figure SI2 IMPS Q-plane plot obtained at SC under $90 \mathrm{~mW} \cdot \mathrm{cm}^{-2}$ blue illumination

Derivation of IMPS model at OC

(a)

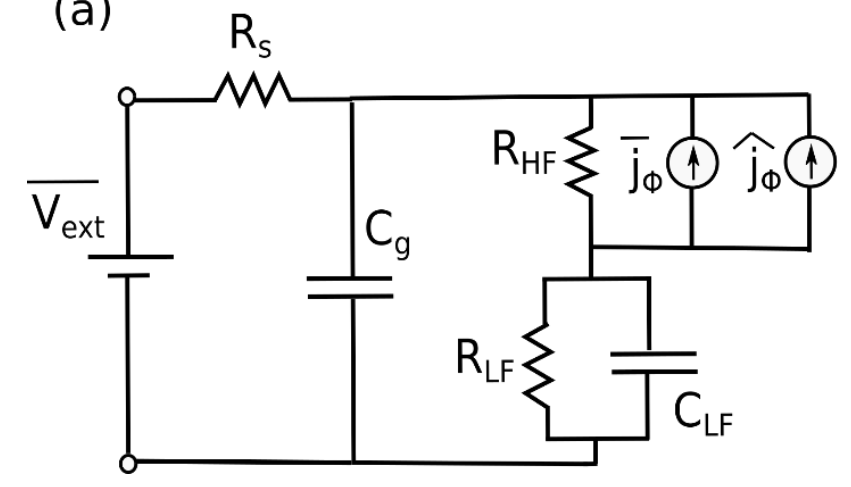

(b)

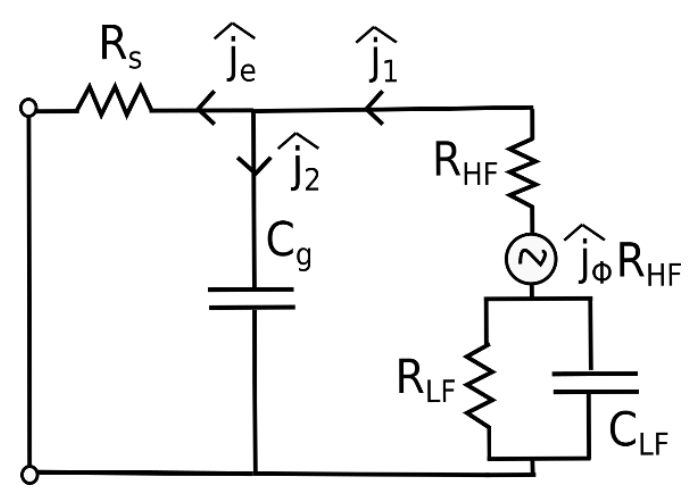

Figure SI3 (a) Perovskite solar cell model upon application of a modulated light intensity including a modulated current source during an IMPS experiment at OC and (b) transformation of modulated current source into a modulated voltage source and replacement of DC voltage and current sources with short and open circuits respectively

In order to determine the IMPS transfer function $Q$, we consider the circuit of Figure SI3a, consisting of an external voltage source $V_{e x t}$ and a DC photon current density $\dot{j}_{\Phi}$, upon which a modulated photon current density $\hat{j}_{\phi}$ is applied. To obtain $Q$, we use the superposition theorem and replace $V_{e x t}$ with a short circuit and $\overline{j_{\Phi}}$ with an open circuit, shown in Figure SI3b. We consider modulated currents $\hat{j}_{1}$ and $\hat{j}_{2}$ through the circuit with a net extracted current $\hat{j}_{e}$ as shown. For OC conditions, we have the validity of Equation 2 in the main text. From Figure SI1, we have 


$$
\hat{j}_{e}=\hat{j}_{1}-\hat{j}_{2}
$$

We also define

$$
\begin{aligned}
& Z_{1}=\frac{1}{i \omega C_{g}} \\
& Z_{2}=\left(\frac{1}{R_{L F}}+i \omega C_{L F}\right)^{-1}
\end{aligned}
$$

Using Kirchoff's laws, we have

$$
\begin{aligned}
& R_{s} \hat{j}_{1}-\left(R_{s}+Z_{1}\right) \hat{j}_{2}=0 \\
& \left(R_{H F}+Z_{2}\right) \hat{j}_{1}+Z_{1} \hat{j}_{2}=\hat{j}_{\phi} R_{H F}
\end{aligned}
$$

Solving Equations SI1, SI4, SI5 and substituting SI2 and SI3 yields

$$
Q(\omega)=\frac{\widehat{j}_{e}}{\widehat{j}_{\phi}}=\left(1+\frac{R_{s}}{R_{H F}}+i \omega R_{s} C_{g}+\left[\frac{R_{L F}}{R_{H F}}\right]\left[\frac{1+i \omega R_{s} C_{g}}{1+i \omega R_{L F} C_{L F}}\right]\right)^{-1}
$$

\section{Derivation of extended IMPS model at OC}
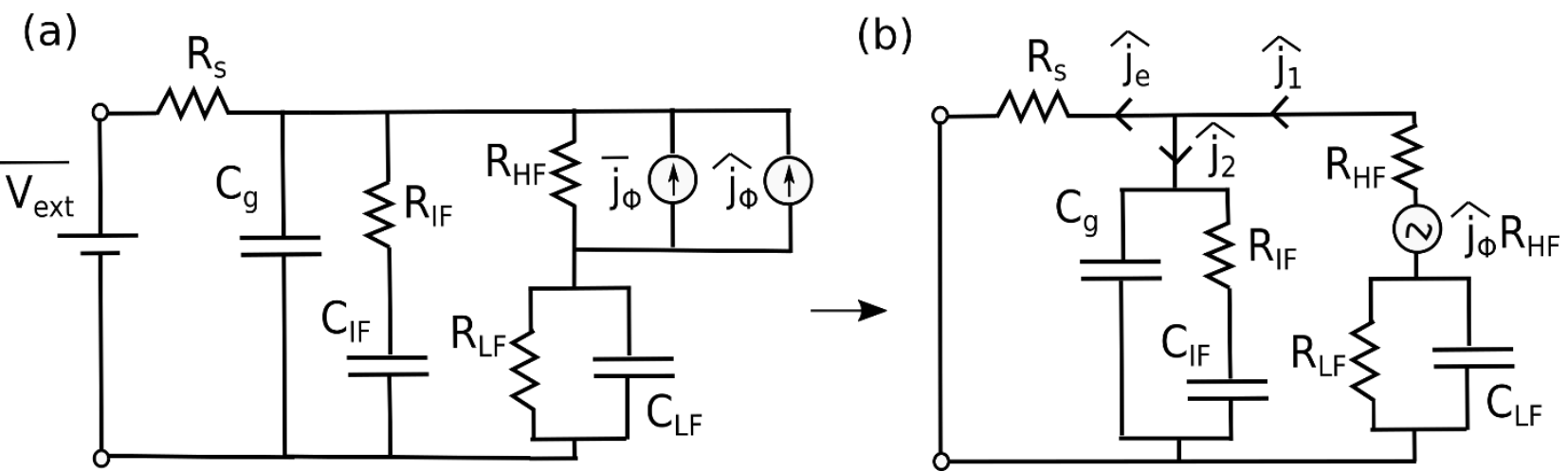

Figure SI4 (a) Extended perovskite solar cell model including intermediate frequency resistance and capacitance, upon application of a modulated light intensity represented by a modulated current source during an IMPS experiment at OC and (b) transformation of modulated current source into a modulated voltage source and replacement of DC voltage and current sources with short and open circuits respectively

From Figure SI4b, we can define

$$
Z_{1}=\left(i \omega C_{g}+\frac{1}{R_{I F}+\frac{1}{i \omega C_{I F}}}\right)^{-1}
$$


While $Z_{2}$ remains the same as in Equation SI3. Solving Equations SI1, SI4, SI5 and substituing SI3 and SI7 yields

$Q(\omega)=\left(1+\frac{R_{s}}{R_{H F}}+i \omega R_{s} C_{g}+\frac{1}{\frac{R_{I F}}{R_{s}}+\frac{1}{i \omega R_{s} C_{I F}}}+\left[\frac{R_{L F}}{R_{H F}}\right]\left[\frac{1}{1+i \omega R_{L F} C_{L F}}\right]\left[1+i \omega R_{s} C_{g}+\frac{1}{\frac{R_{I F}}{R_{s}}+\frac{1}{i \omega R_{s} C_{I F}}}\right]\right)^{-1}$

This yields the three arcs shown in Figure $4 \mathrm{~b}$ with the time constants given by

$$
\begin{aligned}
& \tau_{H F}=R_{s} C_{g} \\
& \tau_{I F}=R_{s} C_{I F} \\
& \tau_{L F}=R_{L F} C_{L F}
\end{aligned}
$$

With the intercepts on the real axis given by

$$
\begin{aligned}
& Q^{\prime}(0)=\left(1+\frac{R_{s}+R_{L F}}{R_{H F}}\right)^{-1} \\
& Q_{I F}^{\prime}=\left(1+\frac{R_{s}}{R_{H F}}\right)^{-1} \\
& Q_{H F}^{\prime}=\left(1+\frac{R_{s}}{R_{H F}}+\frac{R_{s}}{R_{I F}}\right)^{-1}
\end{aligned}
$$

The IS transfer function for the circuit of Figure SI4 is given by

$$
Z(\omega)=R_{s}+\left(i \omega C_{g}+\frac{1}{R_{I F}+\frac{1}{i \omega C_{I F}}}+\frac{1}{R_{H F}+\left[\frac{1}{R_{L F}}+i \omega C_{L F}\right]^{-1}}\right)^{-1}
$$

The corresponding time constants are given by

$$
\begin{aligned}
& \tau_{H F}=R_{H F} C_{g} \\
& \tau_{I F}=R_{I F} C_{I F} \\
& \tau_{L F}=R_{L F} C_{L F}
\end{aligned}
$$

\section{References}

1. Saliba, M.; Correa-Baena, J.-P.; Wolff, C. M.; Stolterfoht, M.; Phung, N.; Albrecht, S.; Neher, D.; Abate, A. How to Make over 20\% Efficient Perovskite Solar Cells in Regular ( $\mathrm{n}-\mathrm{i}-\mathrm{p})$ and Inverted $(\mathrm{p}-\mathrm{i}-\mathrm{n})$ Architectures. Chemistry of Materials 2018, 30 (13), 4193-4201. 


\section{General conclusions}

Irrespective of the large deviation in stability and performance of perovskite solar cells based on materials and methods, there exist several key aspects of the rich and varied optoelectronic response of perovskite solar cells (PSC) which are generally reproduced and point to the underlying device operation mechanisms involved. These features are directly associated to the mixed electronic-ionic conduction in the material, which yields characteristic process timescales ranging from nanoseconds to seconds. The first important observation is a strong electrical hysteresis in the current-voltage $(j-V)$ curve that depends on experimental parameters of bias voltage, light intensity and scan speed and direction. This hysteresis is strongly related to the nature of the electron selective contact (ESL) used, generally maximized for $\mathrm{TiO}_{2}$ and minimized for organic contacts such as PCBM. This hysteresis is reflected in an important characterization tool of the solar cell, the external quantum efficiency measurement, which makes identification of the stabilized performance of the perovskite solar cell quite difficult. In addition, capacitance data from Impedance spectroscopy (IS) measurements show a large capacitance of the order of milliFarads that scales with both applied forward bias and light intensity, much larger than an ionic Helmholtz capacitance. This capacitance is also strongly dependent on the nature of the contacts used, again maximized for $\mathrm{TiO}_{2}$ and minimized for organic contacts such as PCBM, indicating its interfacial origin. The characteristic timescale of this capacitance is within milliseconds-seconds and hence, its mechanisms are directly responsible for the observed slow timescale phenomena. A direct observation of the role of this capacitance on recombination in the PSC is seen from opencircuit voltage $\left(V_{o c}\right)$ decay measurements, where the $V_{o c}$ shows a sharp drop upon switching off the light and then diminishes gradually to a non-zero value over several seconds. This experiment showed that the capacitance at the $\mathrm{TiO}_{2} /$ perovskite interface is related to the accumulation of not only ions but also electronic carriers, which shows a fast recombination at the interface followed by slow recombination at the rate of the drift of ions away from this interface. Charge extraction in PSCs from Intensity Modulated Photocurrent Spectroscopy (IMPS) measurements has also been seen to be slow, on timescales similar to the low frequency (LF) phenomena in IS and $V_{o c}$ decay measurements. However, apart from the slow processes discussed above, the PSC shows excellent and fast separation and transport of electronic carriers within the perovskite, with negligibly small exciton binding energies and very large diffusion lengths much larger than the thickness of the device.

Hence, it can be understood that the role of the interfaces between the perovskite and its selective contacts, especially the electron selective contact, is crucial to guide the performance of the PSC. Upon photogeneration, the electrons and holes are driven efficiently towards their respective selective contacts. At the same time, the ions in the PSC are also driven towards the contacts to balance the net charge. This creates a net 
charge density consisting of both electronic and ionic charge that control the extraction and recombination rates at the interface in a $j-V$ measurement.

Based on these physical observations, a device model and equivalent circuit of the PSC can be developed. Current models in the literature model the perovskite as an intrinsic absorber with a built-in electrical field through the device. This electrical field is the result of the equilibration between the different workfunctions (WF) of the selective contacts. At equilibrium conditions, this electrical field causes the ions to drift to opposite contacts and shield the built-in field. This creates a bulk field-free region and sharp potential drops at the contacts. Upon the application of a forward (reverse) bias, this bulk region is either tilted unfavorably (favorably) to collect photogenerated charges. This charge collectionbased model imposes a strong correlation between the selective contact WF and the output photovoltage of the device as transport depends entirely on the electrical field, which has not been clarified. It is able to reproduce some aspects of the unique hysteresis trends of the PSCs and their dependence on experimental parameters but does not capture the essence of the physical operation of the PSC such as the origin of the large capacitance and the persistent electrostatic potential observed from $V_{o c}$ decay experiments, as discussed earlier. Furthermore, the large diffusion lengths observed make it unclear as to why the perovskite absorber is modelled as an intrinsic semiconductor that requires a net electric field to transport photogenerated charges, similar to insulators.

Therefore, this thesis aims to develop a holistic model of operation of the PSC, culminating in an equivalent circuit (EC) that can reproduce the response of several kinetic measurements, namely the various hysteretic trends and their dependence on experimental parameters in a $\boldsymbol{j}-\boldsymbol{V}$ curve, followed by modelling and connecting the response of the small perturbation methods of IS and IMPS. These will provide an identification and understanding of the different kinetic pathways of recombination and charge accumulation in the PSCs that culminate in the pseudo steady-state response.

The model starts from a point of strong polarization of the compact $\mathrm{TiO}_{2}$ (c$\mathrm{TiO}_{2}$ )/perovskite interface upon the application of a large forward bias, close to $V_{o c}$. At such conditions, the large density of electrons on the $\mathrm{TiO}_{2}$ side brings a density of cations and majority holes to this interface, forming an accumulation layer that creates a net upward band bending or electrostatic potential. The accumulation of electronic carriers in addition to ions explains the increase in capacitance with light intensity. Upon cycling the external voltage, the response of the electrostatic potential is governed by the slow kinetics of the cations in the order of seconds that drift away from this interface. By developing an equation for the evolution of this electrostatic potential and accounting for the capacitive discharge of accumulated charges, several hysteretic trends, including the observation of a 'bump' in photocurrent close to $V_{o c}$, capacitive behavior with respect to scan rate, apparent excess photocurrent value, apparent shunt resistance effect and their dependence on forward bias voltage and scan rates were explained.

However, it was soon realized that these variations in collected current were typical of 
lower efficiency PSCs. It is expected that for PSCs with high efficiencies (15\% and above), the collected current and FFs are very stable, with mainly variations in the obtained $V_{o c}$. This was exemplified in the hysteresis trends of printable mesoscopic PSCs with a $\mathrm{ZrO}_{2}$ framework and Carbon counter electrode. These devices showed a variation from normal to inverted hysteresis upon reducing the thickness of the $\mathrm{c}-\mathrm{TiO}_{2}$ layer, where normal (inverted) hysteresis is defined as a larger (smaller) $V_{o c}$ in the forward to reverse bias (FR) scan than the reverse to forward bias (RF) scan. Therefore, the model was extended to account for recombination of the accumulated holes on the perovskite side with the electrons in the $\mathrm{TiO}_{2}$ side. This extra recombination pathway is strongly dependent on the thickness of the $\mathrm{c}-\mathrm{TiO}_{2}$ layer, which sustains the accumulated charge, and is enhanced for smaller thicknesses. Therefore, at large forward biases, in a FR scan, the $V_{o c}$ is determined by the amount of accumulated charge. For PSCs with smaller c- $\mathrm{TiO}_{2}$ thickness, the interfacial recombination is enhanced, leading to a reduction in the $V_{o c}$ as compared to a sample with thicker $\mathrm{c}-\mathrm{TiO}_{2}$. During the RF scan, the slow kinetics of the ions prevents the build-up of charge to form an accumulation layer and hence, the $V_{o c}$ in this case is governed by bulk recombination and to a much lesser extent, surface recombination. Therefore, the combination of charge accumulation and interfacial recombination at the $\mathrm{c}-\mathrm{TiO}_{2} /$ perovskite interface in a FR scan and the slow ion kinetics in the RF scan qualitatively yield the transition from normal to inverted hysteresis upon reducing the thickness of the $\mathrm{c}-\mathrm{TiO}_{2}$ layer for high efficiency, stable PSCs.

Thus far, it is seen that interfacial polarization of charge within the perovskite itself and their subsequent release or recombination control the output parameters of the $j-V$ performance of the PSC, which is strongly dependent on the nature of the contacts. However, do the contacts also play a role in the transport of photogenerated charges due to a built-in electrical field? In order to answer this question, several high performance PSCs (18-20\% PCE) in a regular configuration were prepared with each selective layer of the ESL removed sequentially. It was found that the output $V_{o c}$ for ESLs with almost a 1 $\mathrm{eV}$ difference in their WFs were remarkably similar. These cells also showed identical short-circuit currents $\left(j_{s c}\right)$, with the only varying factor being the FF. These results clearly showed that there is no correlation between the selective contact WF and the output photovoltage, indicated that the transport of photogenerated carriers within the perovskite is not due to the establishment of a built-in electrical field through the absorber due to difference in WF of the contacts. This leads to the conclusion that any WF difference of the contacts is absorbed in a small layer at the selective contact/perovskite interface, similar to a highly doped semiconductor. Therefore, the transport of photogenerated carriers in high efficiency PSCs is predominantly diffusive.

The picture painted so far consists of photogenerated charges diffusing efficiently through the perovskite absorber, where they reach the selective contact/perovskite interface that contains sharp potential drops. The majority holes then accumulate along with cations at the ESL/perovskite interface depending upon applied bias. The next step was to understand how the photogenerated charges are extracted from the absorber to the 
selective contacts to yield the measured current and which mechanisms create a limiting effect, if any. Therefore, IMPS measurements were carried out to understand the mechanisms of charge extraction in a PSC, as they are a reflection of the frequencydependent external quantum efficiency (EQE).

It was observed that the slow timescales of response of the order of millisecondsseconds observed from several experiments such as IS and $V_{o c}$ decay were also observed in IMPS measurements. The Q-plane response showed a singular reduction in the EQE of the PSC at such frequencies. By development of a suitable EC, it was understood that the reduction in the EQE was related to the large LF capacitance and its corresponding resistance that is outside the photovoltage generated by incident light, in effect acting like a series resistance at very low frequencies. The timescale of this detrimental response means that standard methods of measurement of the EQE, that usually involve the input photon flux modulated by a chopper that is operating between $10-200 \mathrm{~Hz}$, can be highly erroneous in the case of PSCs. In the case of traditional solar cells such as Silicon, the response is much faster than the frequency of the modulation. However, for the PSCs, due to the slow LF process, it was confirmed experimentally from both direct measurements using a chopper and IMPS measurements that the EQE showed a variation of almost $10 \%$ between $14-500 \mathrm{~Hz}$. This explained the large variation in EQE and hence $j_{s c}$ values that are obtained from integration of the EQE for PSCs, further indicating the need to establish rigid protocols to control deviations in EQE measurements. These results further established the importance of the selective contacts in controlling the device performance of a PSC.

Based on these insights regarding charge accumulation, recombination, transport and extraction, the final step was to develop an effective EC that reflected the information gained from the insights from the previous sections and which can accurately reproduce the results of multiple small perturbation techniques such as IS and IMPS, since the underlying EC is essentially the same.

Therefore, IS and IMPS measurements were carried out on stable and efficient Bromide-based PSCs. It was observed that while IS measurements yield two characteristic processes in a Z-plane plot, IMPS measurements show an additional intermediate frequency (IF) process of the order of a few Hz. Therefore, a suitable EC was developed that incorporated this extra IF process, which is akin to the charging of an IF capacitor through a corresponding IF resistance. From theoretical considerations and simulations, it was found that the IF process is invisible in an IS Z-plane plot due to the similarity of its characteristic time with the LF process regularly observed. In the case of IMPS measurements, the time constants for each process are formed by the product of the series resistance and associated capacitors along the lines of the EC and hence, the IF and LF processes are decoupled.

The discovery of the extra IF elements in the EC of the PSC has significant ramifications regarding its operation. This is because the IF resistor is in parallel with the recombination resistance and hence, in a $j-V$ scan where the frequency of scanning is 
similar to the time constant of the IF process, at low forward voltages, the recombination resistance becomes very large and essentially allows the IF resistance to dominate the net device resistance. Therefore, this IF process, attributed to charging the perovskite/holeselective contact interface, controls the current response of the PSC at low forward voltages, which explains the large variation in FF observed for PSCs based on materials and methods.

In conclusion, the insights obtained with respect to the PSC operation can be summarized as:

- The selective contacts play a dominant role in the operation of the PSC. Ionic and electronic charge accumulation at the ESL/perovskite interface and their subsequent capacitive discharge control the singular hysteretic trends of the current-voltage curve for low efficiency PSCs. For high efficiency PSCs, these capacitive discharges are minimized, with variations in mainly the output $V_{o c}$ and fill factor that is strongly influenced by recombination of the accumulated charges at either interface.

- Transport of photogenerated carriers in high efficiency PSCs is mainly diffusive. This is due to the fact that the difference in workfunction of the selective contacts is absorbed in a small layer near the respective interfaces, leaving the majority of the bulk of the perovskite absorber electrical field-free. Therefore, there exists no correlation between the WF of the selective contacts and the output $V_{o c}$ of the PSC.

- The developed EC of the PSC does not contain any contributions of electronic transport phenomena, while ionic transport is not observed directly. The EC is dominated by the capacitive and resistive contributions of the selective contact/perovskite interfaces which play a determinant role in its performance. 